\title{
Asymmetric Halocyclizations of 2-Vinylbenzyl Alcohols with Chiral FLPs
}

\author{
Caifang Han, Xiangqing Feng,* and Haifeng Du*
}

Beijing National Laboratory for Molecular Sciences,

CAS Key Laboratory of Molecular Recognition and Function,

Institute of Chemistry, Chinese Academy of Sciences,

Beijing 100190.

\section{Supporting Information}


General consideration: All air-sensitive compounds were handled under an atmosphere of argon or in a nitrogen-filled glovebox. ${ }^{1} \mathrm{H}$ NMR and ${ }^{13} \mathrm{C}$ NMR spectra were recorded on Bruker $\mathrm{AV} 300,400$ or 500 at ambient temperature with $\mathrm{CDCl}_{3}$ as solvent and TMS as internal standard. Chemical shifts $(\delta)$ were given in ppm, referenced to the residual proton resonance of TMS (0), to the carbon resonance of the $\mathrm{CDCl}_{3}$ (77.23). Coupling constants $(J)$ were given in Hertz (Hz). IR spectrums were recorded on Perkin-Elmer-983 spectrometer. Optical rotations were measured with PerkinElmer 341 polarimeter. Single crystal data was collected on a Bruker Smart APEXII CCD diffractometer using graphite monochromated $\mathrm{Cu} \mathrm{K} \alpha$ radiation. Flash column chromatography was performed on silica gel (200-300 mesh). All solvents were purified by conventional methods, distilled before use. Commercially available reagents were used without further purification.

\section{Optimization of the reaction conditions ${ }^{a}$}

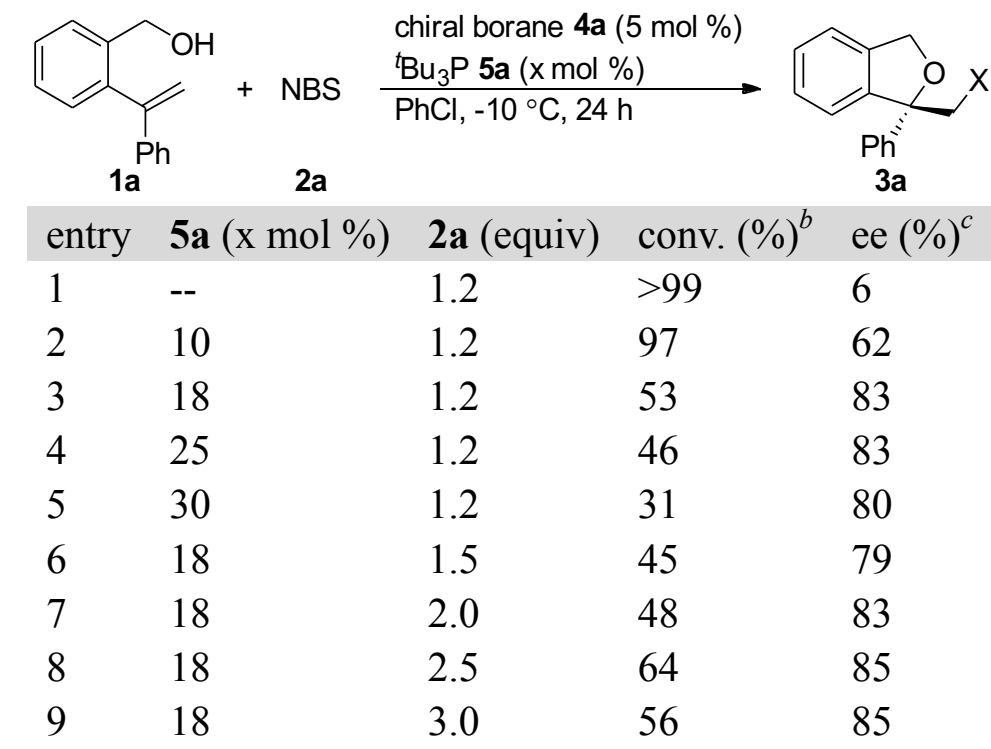

${ }^{a}$ All reactions were carried out with $\mathbf{1 a}(0.10 \mathrm{mmol})$, chiral borane $4 \mathbf{a}(0.005 \mathrm{~mol})$, and ${ }^{t} \mathrm{Bu}_{3} \mathrm{P} \mathbf{5 a}$ (x mol \%) with NBS (2a) in $\mathrm{PhCl}(1.0 \mathrm{~mL})$ at $-10^{\circ} \mathrm{C}$ for $24 \mathrm{~h}$ unless other noted. ${ }^{b}$ Determined by the crude ${ }^{1} \mathrm{H}$ NMR. ${ }^{c}$ Determined by chiral HPLC. 
Some substrates with low reactivity or ee's:
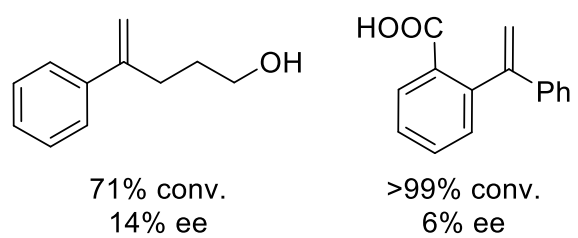<smiles>OCc1ccccc1/C=C/c1ccccc1</smiles><smiles>[R]Nc1ncccc1CO</smiles>

\section{Representative procedure for the synthesis of 1}

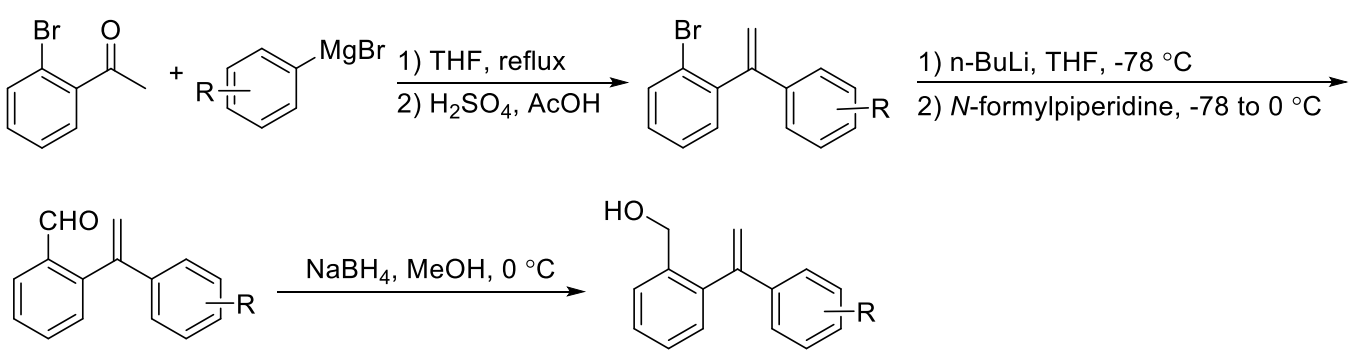

Representative procedure for the synthesis of (2-(1-(4-(tert-butyl)phenyl)vinyl)

phenyl)methanol 1c: A solution of 2'-bromacetophenone (5.0 g, $25 \mathrm{mmol}, 3.4 \mathrm{~mL}, 1.0$ equiv) in THF $(50 \mathrm{~mL})$ was added dropwise to a solution of (4-(tertbutyl)phenyl)magnesium bromide in $\mathrm{Et}_{2} \mathrm{O}(27.5 \mathrm{mmol}, 2 \mathrm{M}, 14.0 \mathrm{~mL}, 1.1$ equiv) at room temperature. The resulting mixture was heated at reflux in an oil bath for $5 \mathrm{~h}$, and then allowed to cool to room temperature. The reaction was quenched by addition of saturated aq. $\mathrm{NH}_{4} \mathrm{Cl}(30 \mathrm{~mL})$. The organic layer was separated, and the aqueous layer was extracted with diethyl ether $(3 \times 40 \mathrm{~mL})$. The combined organic layers were washed with water, dried over $\mathrm{MgSO}_{4}$, filtered and concentrated in vacuo. The crude carbinol was treated with a solution of $\mathrm{H}_{2} \mathrm{SO}_{4}$ in acetic acid $(8 \mathrm{~mL}, 20 \% \mathrm{v} / \mathrm{v})$ at $50{ }^{\circ} \mathrm{C}$ in an oil bath for $1 \mathrm{~h}$. The mixture was then poured into an $\mathrm{Et}_{2} \mathrm{O} /$ water $(1 / 1, \mathrm{v} / \mathrm{v}, 150 \mathrm{~mL})$ twophase system. The organic layer was separated, and the aqueous layer was extracted with diethyl ether $(2 \times 100 \mathrm{~mL})$. The combined organic layers were washed with an aqueous solution of $\mathrm{NaHCO}_{3}(1 \mathrm{M}, 30 \mathrm{~mL})$, dried over $\mathrm{MgSO}_{4}$, filtered and 
concentrated under reduced pressure. The crude product was purified by column chromatography to furnish 1-bromo-2-(1-(4-(tert-butyl)phenyl)vinyl)benzene (5.5 g, $70 \%$ yield) as a pale yellow oil.

The obtained 1-bromo-2-(1-(4-(tert-butyl)phenyl)vinyl)benzene was treated with butyllithium (17.5 mmol, $2.5 \mathrm{M}, 7.0 \mathrm{~mL}, 1.0$ equiv) in THF $(80 \mathrm{~mL})$ at $-78{ }^{\circ} \mathrm{C}$ for $1 \mathrm{~h}$, and the resulting $\alpha$-substituted 2-lithiostyrene was allowed to react with $\mathrm{N}$ formylpiperidine at -78 to $0{ }^{\circ} \mathrm{C}$ for $1 \mathrm{~h}$. The reaction was quenched by addition of saturated aq. $\mathrm{NH}_{4} \mathrm{Cl}(20 \mathrm{~mL})$. The organic layer was separated, and the aqueous layer was extracted with ethyl acetate $(3 \times 30 \mathrm{~mL})$. The combined organic layers were washed with brine, dried over $\mathrm{MgSO}_{4}$, filtered and concentrated under reduced pressure. The crude residue was purified by column chromatography to furnish 2-(1-(4-(tert-butyl) phenyl)vinyl)benzaldehyde ( $2.8 \mathrm{~g}, 60 \%$ yield $)$ as a pale yellow oil.

The reduction of 2-(1-(4-(tert-butyl)phenyl)vinyl)benzaldehyde with sodium borohydride (396 mg, $10.5 \mathrm{mmol}, 1.0$ equiv) in methanol $(40 \mathrm{~mL})$ at $0{ }^{\circ} \mathrm{C}$ for overnight. The reaction was quenched by addition of $1 \% \mathrm{HCl}(20 \mathrm{~mL})$. The mixture was extracted with ethyl acetate $(3 \times 30 \mathrm{~mL})$ and the combined organic layers were washed with brine, dried over $\mathrm{Na}_{2} \mathrm{SO}_{4}$, filtered and concentrated under reduced pressure. The crude residue was purified by column chromatography to furnish (2-(1-(4-(tert-butyl)phenyl)vinyl) phenyl)methanol (1c) (2.0 g, 73\% yield) as a pale yellow solid.

Nicolai, S.; Erard, S.; González, D. F.; Waser, J. Org. Lett. 2010, 12, 384-387;

Kobayashi, K.; Ueyama, T.; Horiuchi, M. Heterocycles 2017, 94, 2065-2079. 
<smiles>C=C(c1ccccc1)c1ccccc1CO</smiles>

(2-(1-Phenylvinyl)phenyl)methanol (1a): pale yellow oil. ${ }^{1} \mathrm{H} \mathrm{NMR}\left(300 \mathrm{MHz}, \mathrm{CDCl}_{3}\right.$, ppm) $\delta 7.50(\mathrm{~d}, J=7.2 \mathrm{~Hz}, 1 \mathrm{H}), 7.43-7.21(\mathrm{~m}, 8 \mathrm{H}), 5.80(\mathrm{~s}, 1 \mathrm{H}), 5.26(\mathrm{~s}, 1 \mathrm{H}), 4.44(\mathrm{~d}$, $J=6.3 \mathrm{~Hz}, 2 \mathrm{H}), 1.40(\mathrm{t}, J=6.3 \mathrm{~Hz}, 1 \mathrm{H}) ;{ }^{13} \mathrm{C} \mathrm{NMR}\left(75 \mathrm{MHz}, \mathrm{CDCl}_{3}, \mathrm{ppm}\right) \delta 148.5$, $140.8,140.7,138.9,130.4,128.7,128.28,128.25,128.2,127.8,126.7,115.8,63.4$.

Kobayashi, K.; Ueyama, T.; Horiuchi, M. Heterocycles 2017, 94, 2065-2079.<smiles>C=C(c1ccc(C)cc1)c1ccccc1CO</smiles>

(2-(1-(p-Tolyl)vinyl)phenyl)methanol (1b): colorless oil. ${ }^{1} \mathrm{H}$ NMR (300 MHz, $\mathrm{CDCl}_{3}$, ppm) $\delta$ 7.58-7.48 (m, 1H), 7.46-7.25 (m, 3H), 7.25-7.10 (m, 4H), $5.79(\mathrm{~d}, J=1.2 \mathrm{~Hz}$ $1 \mathrm{H}), 5.23(\mathrm{~d}, J=1.2 \mathrm{~Hz}, 1 \mathrm{H}), 4.47(\mathrm{~d}, J=6.3 \mathrm{~Hz}, 2 \mathrm{H}), 2.36(\mathrm{~s}, 3 \mathrm{H}), 1.47(\mathrm{t}, J=6.3 \mathrm{~Hz}$, $1 \mathrm{H}) ;{ }^{13} \mathrm{C} \mathrm{NMR}\left(75 \mathrm{MHz}, \mathrm{CDCl}_{3}, \mathrm{ppm}\right) \delta 148.3,140.9,138.8,138.1,138.0,130.4,129.4$, $128.4,128.2,127.8,126.6,114.9,63.5,21.3$.

Kobayashi, K.; Ueyama, T.; Horiuchi, M. Heterocycles 2017, 94, 2065-2079.<smiles>C=C(c1ccc(Br)cc1)c1ccccc1CO</smiles>

(2-(1-(4-(tert-Butyl)phenyl)vinyl)phenyl)methanol (1c): pale yellow solid, m.p. 68$69{ }^{\circ} \mathrm{C}$; IR (film): 3349, 2962, 1613, $1511 \mathrm{~cm}^{-1} ;{ }^{1} \mathrm{H}$ NMR $\left(300 \mathrm{MHz}, \mathrm{CDCl}_{3}, \mathrm{ppm}\right) \delta$ 7.52-7.46 (m, 1H), 7.42-7.28 (m, 4H), 7.28-7.17 (m, 3H), $5.78(\mathrm{~d}, J=1.2 \mathrm{~Hz}, 1 \mathrm{H}), 5.20$ $(\mathrm{d}, J=1.2 \mathrm{~Hz}, 1 \mathrm{H}), 4.46(\mathrm{~d}, J=6.3 \mathrm{~Hz}, 2 \mathrm{H}), 1.41(\mathrm{t}, J=6.3 \mathrm{~Hz}, 1 \mathrm{H}), 1.30(\mathrm{~s}, 9 \mathrm{H}) ;{ }^{13} \mathrm{C}$ NMR (75 MHz, $\left.\mathrm{CDCl}_{3}, \mathrm{ppm}\right) \delta 151.3,148.2,140.9,138.9,137.8,130.4,128.3,128.2$, 
127.8, 126.4 125.6, 115.0, 63.5, 34.8, 31.5; HRMS (APCI) calcd. for $\mathrm{C}_{19} \mathrm{H}_{21} \mathrm{O}(\mathrm{M}-\mathrm{H})$ : 265.1587, Found: 265.1585.<smiles>C=Cc1ccc(C(=C)c2ccccc2CO)cc1</smiles>

(2-(1-(4-Vinylphenyl)vinyl)phenyl)methanol (1d): white solid, m.p. $82-83{ }^{\circ} \mathrm{C}$; IR (film): $3364,2921,1647,848 \mathrm{~cm}^{-1} ;{ }^{1} \mathrm{H}$ NMR $\left(300 \mathrm{MHz}, \mathrm{CDCl}_{3}, \mathrm{ppm}\right) \delta 7.50(\mathrm{~d}, J=$ $6.3 \mathrm{~Hz}, 1 \mathrm{H}), 7.42-7.30(\mathrm{~m}, 4 \mathrm{H}), 7.29-7.21(\mathrm{~m}, 3 \mathrm{H}), 6.69(\mathrm{dd}, J=17.7,11.1 \mathrm{~Hz}, 1 \mathrm{H})$, $5.81(\mathrm{~d}, J=1.2 \mathrm{~Hz}, 1 \mathrm{H}), 5.74(\mathrm{~d}, J=17.1 \mathrm{~Hz}, 1 \mathrm{H}), 5.25(\mathrm{dd}, J=5.4,0.9 \mathrm{~Hz}, 2 \mathrm{H}), 4.44$ $(\mathrm{d}, J=6.3 \mathrm{~Hz}, 2 \mathrm{H}), 1.45(\mathrm{t}, J=6.3 \mathrm{~Hz}, 1 \mathrm{H}) ;{ }^{13} \mathrm{C} \mathrm{NMR}\left(125 \mathrm{MHz}, \mathrm{CDCl}_{3}, \mathrm{ppm}\right) \delta 148.1$, $140.6,140.2,138.9,137.5,136.4,130.4,128.3,128.2,127.8,126.9,126.6,115.7,114.4$, 63.4; HRMS (APCI) calcd. for $\mathrm{C}_{17} \mathrm{H}_{17} \mathrm{O}(\mathrm{M}+\mathrm{H})$ : 237.1274, Found: 237.1272 .<smiles>C=C(c1ccc(-c2ccccc2)cc1)c1ccccc1CO</smiles>

(2-(1-([1,1'-Biphenyl]-4-yl)vinyl)phenyl)methanol (1e): white solid, m.p. $110-111^{\circ} \mathrm{C}$; IR (film): 3336, 1486, $768 \mathrm{~cm}^{-1} ;{ }^{1} \mathrm{H}$ NMR $\left(300 \mathrm{MHz}, \mathrm{CDCl}_{3}, \mathrm{ppm}\right) \delta$ 7.65-7.53 (m, 5H), 7.50-7.28 (m, 8H), $5.89(\mathrm{~d}, J=1.2 \mathrm{~Hz}, 1 \mathrm{H}), 5.31(\mathrm{~d}, J=1.2 \mathrm{~Hz}, 1 \mathrm{H}), 4.52(\mathrm{~d}, J=6.0$ $\mathrm{Hz}, 2 \mathrm{H}), 1.53(\mathrm{t}, J=6.0 \mathrm{~Hz}, 1 \mathrm{H}) ;{ }^{13} \mathrm{C} \mathrm{NMR}\left(125 \mathrm{MHz}, \mathrm{CDCl}_{3}, \mathrm{ppm}\right) \delta 148.0,141.0$, $140.7,140.6,139.7,138.9,130.4,129.0,128.3,128.2,127.9,127.7,127.4,127.2,127.1$ 115.8, 63.5; HRMS (APCI) calcd. for $\mathrm{C}_{21} \mathrm{H}_{17} \mathrm{O}(\mathrm{M}-\mathrm{H})$ : 285.1285, Found: 285.1279.<smiles>C=C(c1ccc(F)cc1)c1ccccc1CO</smiles> 
(2-(1-(4-Fluorophenyl)vinyl)phenyl)methanol (1f): pale yellow oil. ${ }^{1} \mathrm{H}$ NMR (300 $\left.\mathrm{MHz}, \mathrm{CDCl}_{3}, \mathrm{ppm}\right) \delta 7.48(\mathrm{~d}, J=7.2 \mathrm{~Hz}, 1 \mathrm{H}), 7.42-7.28(\mathrm{~m}, 2 \mathrm{H}), 7.27-7.18(\mathrm{~m}, 3 \mathrm{H})$, 7.03-6.91 (m, 2H), $5.72(\mathrm{~s}, 1 \mathrm{H}), 5.21(\mathrm{~s}, 1 \mathrm{H}), 4.41(\mathrm{~s}, 2 \mathrm{H}), 1.65(\mathrm{br}, 1 \mathrm{H}) ;{ }^{13} \mathrm{C}$ NMR $(75$ $\left.\mathrm{MHz}, \mathrm{CDCl}_{3}, \mathrm{ppm}\right) \delta 162.7(\mathrm{~d}, J=246.0 \mathrm{~Hz}), 147.4,140.4,138.8,136.8(\mathrm{~d}, J=3.0$ Hz), 130.3, $128.4(\mathrm{~d}, J=8.3 \mathrm{~Hz}), 128.3,128.1,127.8,115.6(\mathrm{~d}, J=21.8 \mathrm{~Hz}), 115.4$, 63.2; ${ }^{19} \mathrm{~F}$ NMR $\left(376 \mathrm{MHz}, \mathrm{CDCl}_{3}, \mathrm{ppm}\right) \delta-114.1$.

Ha, T. M.; Wang, Q.; Zhu, J. Chem. Commun. 2016, 52, 11100-11103.<smiles>C=C(c1ccc(Cl)cc1)c1ccccc1CO</smiles>

(2-(1-(4-Chlorophenyl)vinyl)phenyl)methanol (1g): colorless oil. ${ }^{1} \mathrm{H}$ NMR (300 $\left.\mathrm{MHz}, \mathrm{CDCl}_{3}, \mathrm{ppm}\right) \delta 7.51(\mathrm{~d}, J=7.5 \mathrm{~Hz}, 1 \mathrm{H}), 7.47-7.16(\mathrm{~m}, 7 \mathrm{H}), 5.79(\mathrm{~s}, 1 \mathrm{H}), 5.27(\mathrm{~s}$, $1 \mathrm{H}), 4.44(\mathrm{~d}, J=6.0 \mathrm{~Hz}, 2 \mathrm{H}), 1.45(\mathrm{t}, J=6.0 \mathrm{~Hz}, 1 \mathrm{H}) ;{ }^{13} \mathrm{C} \mathrm{NMR}\left(75 \mathrm{MHz}, \mathrm{CDCl}_{3}\right.$, ppm) $\delta 147.4,140.1,139.2,138.8,134.1,130.4,128.9,128.4,128.2,128.1,127.9$, $116.3,63.3$

Kobayashi, K.; Ueyama, T.; Horiuchi, M. Heterocycles 2017, 94, 2065-2079.<smiles>C=C(c1ccc2ccccc2c1)c1ccccc1CO</smiles>

(2-(1-(Naphthalen-2-yl)vinyl)phenyl)methanol (1h): white solid, m.p. 101-102 ${ }^{\circ} \mathrm{C}$; IR (film): 3333, 3055, 1615, 1504, $751 \mathrm{~cm}^{-1} ;{ }^{1} \mathrm{H} \mathrm{NMR}\left(300 \mathrm{MHz}, \mathrm{CDCl}_{3}, \mathrm{ppm}\right) \delta 7.84-$ $7.75(\mathrm{~m}, 2 \mathrm{H}), 7.74-7.66(\mathrm{~m}, 1 \mathrm{H}), 7.59-7.49(\mathrm{~m}, 3 \mathrm{H}), 7.48-7.27(\mathrm{~m}, 5 \mathrm{H}), 5.94(\mathrm{~s}, 1 \mathrm{H})$, $5.34(\mathrm{~s}, 1 \mathrm{H}), 4.46(\mathrm{~d}, J=6.0 \mathrm{~Hz}, 2 \mathrm{H}), 1.46(\mathrm{t}, J=6.0 \mathrm{~Hz}, 1 \mathrm{H}) ;{ }^{13} \mathrm{C} \mathrm{NMR}(75 \mathrm{MHz}$, $\left.\mathrm{CDCl}_{3}, \mathrm{ppm}\right) \delta 148.4,140.6,139.0,138.0,133.5,133.2,130.5,128.5,128.4,128.3$, 
128.2, 127.9, 127.8, 126.5, 126.4, 126.2, 124.4, 116.4, 63.4; HRMS (APCI) calcd. for $\mathrm{C}_{19} \mathrm{H}_{15} \mathrm{O}(\mathrm{M}-\mathrm{H}): 259.1128$, Found: 259.1127.<smiles>C=C(c1cccc(C)c1)c1ccccc1CO</smiles>

(2-(1-(m-Tolyl)vinyl)phenyl)methanol (1i): colorless oil. IR (film): 3396, 2921, 1601, 1486, 1035, $772 \mathrm{~cm}^{-1} ;{ }^{1} \mathrm{H}$ NMR (300 MHz, $\left.\mathrm{CDCl}_{3}, \mathrm{ppm}\right) \delta 7.47(\mathrm{~d}, J=7.5 \mathrm{~Hz}, 1 \mathrm{H})$, 7.39-7.27 (m, 2H), 7.27-7.21 (m, 1H), 7.20-7.12 (m, 1H), 7.12-7.00 (m, 3H), $5.76(\mathrm{~d}, J$ $=1.2 \mathrm{~Hz}, 1 \mathrm{H}), 5.21(\mathrm{~d}, J=1.2 \mathrm{~Hz}, 1 \mathrm{H}), 4.41(\mathrm{~s}, 2 \mathrm{H}), 2.30(\mathrm{~s}, 3 \mathrm{H}), 1.65(\mathrm{br}, 1 \mathrm{H}) ;{ }^{13} \mathrm{C}$ NMR (75 MHz, $\left.\mathrm{CDCl}_{3}, \mathrm{ppm}\right) \delta 148.6,140.8,140.7,138.8,138.3,130.3,129.0,128.6$, 128.2, 128.1, 127.7, 127.3, 124.0, 115.7, 63.3, 21.6; HRMS (APCI) calcd. for $\mathrm{C}_{16} \mathrm{H}_{15} \mathrm{O}$ (M-H): 223.1128, Found: 223.1123.<smiles>C=C(c1cccc(C(C)C)c1)c1ccccc1CO</smiles>

(2-(1-(3-Isopropylphenyl)vinyl)phenyl)methanol (1j): colorless oil. IR (film): 3388, 1599, 1459, 1030, $752 \mathrm{~cm}^{-1} ;{ }^{1} \mathrm{H}$ NMR $\left(300 \mathrm{MHz}, \mathrm{CDCl}_{3}, \mathrm{ppm}\right) \delta 7.52(\mathrm{~d}, J=7.5 \mathrm{~Hz}$, 1H), 7.44-7.32 (m, 2H), 7.32-7.17 (m, 4H), 7.11-7.05 (m, 1H), $5.82(\mathrm{~d}, J=1.2 \mathrm{~Hz}, 1 \mathrm{H})$, $5.27(\mathrm{~d}, J=1.2 \mathrm{~Hz}, 1 \mathrm{H}), 4.47$ (d, $J=6.3 \mathrm{~Hz}, 2 \mathrm{H}), 2.89$ (hept, $J=6.9 \mathrm{~Hz}, 1 \mathrm{H}), 1.49$ (t, $J=6.3 \mathrm{~Hz}, 1 \mathrm{H}), 1.25(\mathrm{~d}, J=6.9 \mathrm{~Hz}, 6 \mathrm{H}) ;{ }^{13} \mathrm{C} \mathrm{NMR}\left(75 \mathrm{MHz}, \mathrm{CDCl}_{3}, \mathrm{ppm}\right) \delta 149.4$, $148.8,140.9,140.8,138.9,130.4,128.7,128.4,128.2,127.8,126.2,124.9,124.5,115.6$, 63.5, 34.3, 24.2; HRMS (APCI) calcd. for $\mathrm{C}_{18} \mathrm{H}_{19} \mathrm{O}$ (M-H): 251.1430, Found: 251.1432 .<smiles>C=C(c1cccc(C(C)(C)C)c1)c1ccccc1CO</smiles> 
(2-(1-(3-(tert-Butyl)phenyl)vinyl)phenyl)methanol (1k): colorless oil. IR (film): 3392, 1598, 1484, 1031, $764 \mathrm{~cm}^{-1} ;{ }^{1} \mathrm{H}$ NMR (300 MHz, $\left.\mathrm{CDCl}_{3}, \mathrm{ppm}\right) \delta 7.49$ (d, $J=7.5$ $\mathrm{Hz}, 1 \mathrm{H}), 7.42-7.18(\mathrm{~m}, 6 \mathrm{H}), 7.06-6.99(\mathrm{~m}, 1 \mathrm{H}), 5.79(\mathrm{~d}, J=1.2 \mathrm{~Hz}, 1 \mathrm{H}), 5.24(\mathrm{~d}, J=$ $1.2 \mathrm{~Hz}, 1 \mathrm{H}), 4.43(\mathrm{~d}, J=6.3 \mathrm{~Hz}, 2 \mathrm{H}), 1.43(\mathrm{t}, J=6.3 \mathrm{~Hz}, 1 \mathrm{H}), 1.28(\mathrm{~s}, 9 \mathrm{H}) ;{ }^{13} \mathrm{C} \mathrm{NMR}$ $\left(75 \mathrm{MHz}, \mathrm{CDCl}_{3}, \mathrm{ppm}\right) \delta 151.6,149.0,141.0,140.6,138.9,130.4,128.4,128.3,128.2$, 127.8, 125.3, 124.2, 123.6, 115.5, 63.5, 34.9, 31.5; HRMS (APCI) calcd. for $\mathrm{C}_{19} \mathrm{H}_{21} \mathrm{O}$ (M-H): 265.1587, Found: 265.1588.<smiles>C=C(c1cccc(-c2ccccc2)c1)c1ccccc1CO</smiles>

(2-(1-([1,1'-Biphenyl]-3-yl)vinyl)phenyl)methanol (11): colorless oil. IR (film): 3419, 1597, 1478, 1027, $760 \mathrm{~cm}^{-1} ;{ }^{1} \mathrm{H}$ NMR (300 MHz, $\left.\mathrm{CDCl}_{3}, \mathrm{ppm}\right) \delta$ 7.58-7.47 (m, 5H), 7.46-7.19 (m, 8H), $5.86(\mathrm{~d}, J=0.9 \mathrm{~Hz}, 1 \mathrm{H}), 5.30(\mathrm{~d}, J=1.2 \mathrm{~Hz}, 1 \mathrm{H}), 4.48(\mathrm{~d}, J=6.0$ $\mathrm{Hz}, 2 \mathrm{H}), 1.53(\mathrm{t}, J=6.0 \mathrm{~Hz}, 1 \mathrm{H}) ;{ }^{13} \mathrm{C} \mathrm{NMR}\left(75 \mathrm{MHz}, \mathrm{CDCl}_{3}, \mathrm{ppm}\right) \delta 148.5,141.8$, $141.4,141.1,140.6,138.9,130.4,129.1,129.0,128.3,128.2,127.8,127.6,127.4,127.1$, 125.8, 125.5, 116.2, 63.4; HRMS (APCI) calcd. for $\mathrm{C}_{21} \mathrm{H}_{17} \mathrm{O}(\mathrm{M}-\mathrm{H})$ : 285.1285, Found: 285.1279 .<smiles>C=C(c1cccc(F)c1)c1ccccc1CO</smiles>

(2-(1-(3-Fluorophenyl)vinyl)phenyl)methanol (1m): colorless oil. IR (film): 3373, 1611, 1580, 1034, $789 \mathrm{~cm}^{-1} ;{ }^{1} \mathrm{H}$ NMR $\left(300 \mathrm{MHz}, \mathrm{CDCl}_{3}, \mathrm{ppm}\right) \delta 7.47$ (d, J=7.2 Hz, 1H), 7.40-7.16 (m, 4H), 7.03-6.89 (m, 3H), $5.79(\mathrm{~s}, 1 \mathrm{H}), 5.26(\mathrm{~s}, 1 \mathrm{H}), 4.39(\mathrm{~d}, J=5.1$ $\mathrm{Hz}, 2 \mathrm{H}), 1.84(\mathrm{br}, 1 \mathrm{H}) ;{ }^{13} \mathrm{C} \mathrm{NMR}\left(75 \mathrm{MHz}, \mathrm{CDCl}_{3}, \mathrm{ppm}\right) \delta 163.1(\mathrm{~d}, J=244.5 \mathrm{~Hz})$, 
$147.3(\mathrm{~d}, J=2.3 \mathrm{~Hz}), 143.0(\mathrm{~d}, J=7.5 \mathrm{~Hz}), 139.8,138.8,130.3,130.1(\mathrm{~d}, J=8.3 \mathrm{~Hz})$, 128.4, 128.0, 127.8, $122.4(\mathrm{~d}, J=2.3 \mathrm{~Hz}), 116.8,114.9(\mathrm{~d}, J=21.0 \mathrm{~Hz}), 113.5(\mathrm{~d}, J=$ 21.8 Hz), 63.1; ${ }^{19} \mathrm{~F}$ NMR (376 MHz, $\left.\mathrm{CDCl}_{3}, \mathrm{ppm}\right) \delta-111.5$; HRMS (APCI) calcd. for $\mathrm{C}_{15} \mathrm{H}_{12} \mathrm{OF}$ (M-H): 227.0878, Found: 227.0871.<smiles>C=C(c1cc(C)cc(C)c1)c1ccccc1CO</smiles>

(2-(1-(3,5-Dimethylphenyl)vinyl)phenyl)methanol (1n): colorless oil. IR (film): $3418,1598,1449,1036,770 \mathrm{~cm}^{-1} ;{ }^{1} \mathrm{H}$ NMR $\left(300 \mathrm{MHz}, \mathrm{CDCl}_{3}, \mathrm{ppm}\right) \delta 7.47(\mathrm{~d}, J=7.2$ $\mathrm{Hz}, 1 \mathrm{H}), 7.40-7.28(\mathrm{~m}, 2 \mathrm{H}), 7.27-7.21(\mathrm{~m}, 1 \mathrm{H}), 6.94-6.84(\mathrm{~m}, 3 \mathrm{H}), 5.74(\mathrm{~d}, J=1.5 \mathrm{~Hz}$, 1H), $5.20(\mathrm{~d}, J=1.2 \mathrm{~Hz}, 1 \mathrm{H}), 4.43(\mathrm{~d}, J=6.0 \mathrm{~Hz}, 2 \mathrm{H}), 2.26(\mathrm{~s}, 6 \mathrm{H}), 1.52(\mathrm{br}, 1 \mathrm{H}) ;{ }^{13} \mathrm{C}$ NMR (75 MHz, $\left.\mathrm{CDCl}_{3}, \mathrm{ppm}\right) \delta 148.8,141.0,140.9,138.8,138.2,130.4,130.0,128.3$, 128.1, 127.7, 124.6, 115.6, 63.5, 21.5; HRMS (APCI) calcd. for $\mathrm{C}_{17} \mathrm{H}_{17} \mathrm{O}(\mathrm{M}-\mathrm{H})$ : 237.1274, Found: 237.1274 .<smiles>C=C(c1cccs1)c1ccccc1CO</smiles>

(2-(1-(Thiophen-2-yl)vinyl)phenyl)methanol (10): yellow oil; IR (film): 3388, 2922, 1610, 1023, $702 \mathrm{~cm}^{-1} ;{ }^{1} \mathrm{H}$ NMR (300 MHz, $\left.\mathrm{CDCl}_{3}, \mathrm{ppm}\right) \delta 7.53(\mathrm{~d}, J=7.5 \mathrm{~Hz}, 1 \mathrm{H})$, 7.44-7.17 (m, 4H), 6.94-6.86 (m, 1H), 6.66-6.58 (m, 1H), $5.78(\mathrm{~s}, 1 \mathrm{H}), 5.08(\mathrm{~s}, 1 \mathrm{H})$, $4.58(\mathrm{~d}, J=5.7 \mathrm{~Hz}, 2 \mathrm{H}), 1.57(\mathrm{t}, J=6.0 \mathrm{~Hz}, 1 \mathrm{H}) ;{ }^{13} \mathrm{C} \mathrm{NMR}\left(100 \mathrm{MHz}, \mathrm{CDCl}_{3}, \mathrm{ppm}\right) \delta$ $145.4,141.9,139.9,138.9,129.9,128.5,128.3,127.8,127.7,126.4,125.7,114.2,63.3$; HRMS (APCI) calcd. for $\mathrm{C}_{13} \mathrm{H}_{13} \mathrm{OS}(\mathrm{M}+\mathrm{H}): 217.0682$, Found: 217.0684. 
<smiles>C=C(c1ccccc1)c1ccc(F)cc1CO</smiles>

(5-Fluoro-2-(1-phenylvinyl)phenyl)methanol (1p): pale yellow oil. IR (film): 3368, 2921, 1606, 1494, 909, $781 \mathrm{~cm}^{-1}$; ${ }^{1} \mathrm{H}$ NMR $\left(300 \mathrm{MHz}, \mathrm{CDCl}_{3}, \mathrm{ppm}\right) \delta$ 7.36-7.17 (m, $7 \mathrm{H}), 7.06-6.96(\mathrm{~m}, 1 \mathrm{H}), 5.78(\mathrm{~d}, J=1.2 \mathrm{~Hz}, 1 \mathrm{H}), 5.22(\mathrm{~d}, J=0.9 \mathrm{~Hz}, 1 \mathrm{H}), 4.40(\mathrm{~d}, J=$ $4.8 \mathrm{~Hz}, 2 \mathrm{H}), 1.50(\mathrm{t}, J=5.7 \mathrm{~Hz}, 1 \mathrm{H}) ;{ }^{13} \mathrm{C} \mathrm{NMR}\left(100 \mathrm{MHz}, \mathrm{CDCl}_{3}, \mathrm{ppm}\right) \delta 162.9(\mathrm{~d}, J$ $=244.7 \mathrm{~Hz}), 147.6,141.4(\mathrm{~d}, J=6.9 \mathrm{~Hz}), 140.6,136.2,131.9(\mathrm{~d}, J=7.8 \mathrm{~Hz}), 128.8$, $128.3,126.7,116.2,114.6(\mathrm{~d}, J=22.2 \mathrm{~Hz}), 114.3(\mathrm{~d}, J=20.9 \mathrm{~Hz}), 62.9 ;{ }^{19} \mathrm{~F}$ NMR $(376$ $\left.\mathrm{MHz}, \mathrm{CDCl}_{3}, \mathrm{ppm}\right) \delta$-114.2. HRMS (ESI) calcd. for $\mathrm{C}_{15} \mathrm{H}_{12} \mathrm{FO}(\mathrm{M}-\mathrm{H}): 227.0878$, Found: 227.0875.<smiles>C=C(c1ccc(F)cc1)c1ccc(C#N)cc1CO</smiles>

4-(1-(4-Fluorophenyl)vinyl)-3-(hydroxymethyl)benzonitrile (1q): colorless oil. ${ }^{1} \mathrm{H}$ NMR (400 MHz, $\left.\mathrm{CDCl}_{3}, \mathrm{ppm}\right) \delta 7.89(\mathrm{~s}, 1 \mathrm{H}), 7.62(\mathrm{~d}, J=7.6 \mathrm{~Hz}, 1 \mathrm{H}), 7.34(\mathrm{~d}, J=8.0$ $\mathrm{Hz}, 1 \mathrm{H}), 7.23-7.14(\mathrm{~m}, 2 \mathrm{H}), 7.07-6.95(\mathrm{~m}, 2 \mathrm{H}), 5.80(\mathrm{~s}, 1 \mathrm{H}), 5.24(\mathrm{~s}, 1 \mathrm{H}), 4.46(\mathrm{~s}, 2 \mathrm{H})$, $1.70(\mathrm{br}, 1 \mathrm{H}) ;{ }^{13} \mathrm{C} \mathrm{NMR}\left(125 \mathrm{MHz}, \mathrm{CDCl}_{3}, \mathrm{ppm}\right) \delta 163.0(\mathrm{~d}, J=247.5 \mathrm{~Hz}), 146.0$ 144.6, 140.5, $135.6(\mathrm{~d}, J=3.8 \mathrm{~Hz}), 131.24,131.20,130.9,128.4(\mathrm{~d}, J=7.5 \mathrm{~Hz}), 119.0$, 116.6, $115.9(\mathrm{~d}, J=21.3 \mathrm{~Hz}), 112.3,62.1 ;{ }^{19} \mathrm{~F} \mathrm{NMR}\left(376 \mathrm{MHz}, \mathrm{CDCl}_{3}, \mathrm{ppm}\right) \delta-113.0$. Alamillo-Ferrer, C.; Karabourniotis-Sotti, M.; Kennedy, A. R.; Campbell, M.; Tomkinson, N. C. O. Org. Lett. 2016, 18, 3102-3105.<smiles>C=C(c1ccccc1)c1cc(F)ccc1CO</smiles> 
(4-Fluoro-2-(1-phenylvinyl)phenyl)methanol (1r): pale yellow oil. IR (film): 3320, 2927, 1609, 1494, 909, $781 \mathrm{~cm}^{-1} ;{ }^{1} \mathrm{H}$ NMR $\left(300 \mathrm{MHz}, \mathrm{CDCl}_{3}, \mathrm{ppm}\right) \delta 7.46$ (dd, $J=8.4$, 5.7 Hz, 1H), 7.36-7.22 (m, 5H), 7.11-7.02 (m, 1H), $6.98(\mathrm{dd}, J=9.3,2.7 \mathrm{~Hz}, 1 \mathrm{H}), 5.81$ $(\mathrm{d}, J=0.9 \mathrm{~Hz}, 1 \mathrm{H}), 5.26(\mathrm{~d}, J=0.9 \mathrm{~Hz}, 1 \mathrm{H}), 4.38(\mathrm{~d}, J=3.0 \mathrm{~Hz}, 2 \mathrm{H}), 1.44(\mathrm{br}, 1 \mathrm{H})$;

${ }^{13} \mathrm{C}$ NMR $\left(100 \mathrm{MHz}, \mathrm{CDCl}_{3}, \mathrm{ppm}\right) \delta 162.2(\mathrm{~d}, J=245.0 \mathrm{~Hz}), 147.6,142.7(\mathrm{~d}, J=8.0$ $\mathrm{Hz}), 140.2,134.8(\mathrm{~d}, J=3.0 \mathrm{~Hz}), 130.2(\mathrm{~d}, J=9.0 \mathrm{~Hz}), 128.8,128.4,126.7,117.2(\mathrm{~d}$, $J=22.0 \mathrm{~Hz}), 116.3,114.9(\mathrm{~d}, J=20.0 \mathrm{~Hz}), 62.7 ;{ }^{19} \mathrm{~F} \mathrm{NMR}\left(376 \mathrm{MHz}, \mathrm{CDCl}_{3}, \mathrm{ppm}\right) \delta$ -115.2; HRMS (ESI) calcd. for $\mathrm{C}_{15} \mathrm{H}_{12} \mathrm{FO}(\mathrm{M}-\mathrm{H})$ : 227.0878, Found: 227.0874.

\section{Representative procedure for the asymmetric bromocyclization of 1a (Scheme 4):}

To a tube $(15 \mathrm{~mL}), \mathrm{HB}\left(\mathrm{C}_{6} \mathrm{~F}_{5}\right)_{2}(0.0138 \mathrm{~g}, 0.040 \mathrm{mmol})$, chiral diene $6 \mathbf{a}(0.0137 \mathrm{~g}, 0.020$ $\mathrm{mmol})$, and dry chlorobenzene $(1.2 \mathrm{~mL})$ were added in a nitrogen atmosphere glovebox. The resulting mixture was stirred for $5 \mathrm{~min}$ at room temperature followed by addition of ${ }^{t} \mathrm{Bu}_{3} \mathrm{P}(0.0146 \mathrm{~g}, 0.072 \mathrm{mmol})$. After stirring for $5 \mathrm{~min}, \mathbf{1 a}(0.0841 \mathrm{~g}, 0.4 \mathrm{mmol})$ and NBS (0.1780 g, $1.0 \mathrm{mmol})$ were added. Then the tube was sealed, and the reaction mixture was stirred at $0{ }^{\circ} \mathrm{C}$ for $24 \mathrm{~h}$. The reaction mixture was added triethylamine $(0.2$ $\mathrm{mL}$ ). The resulting solution was concentrated, and the residue was purified by column chromatography on silica gel (petroleum ether/ethyl acetate $=50 / 1$ ) to afford the desired (R)-3a as a white solid $(0.1100 \mathrm{~g}, 95 \%$ yield, $84 \%$ ee)

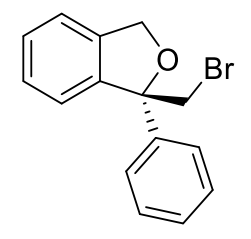

(R)-1-(Bromomethyl)-1-phenyl-1,3-dihydroisobenzofuran (3a): The Scheme 4 was followed by using (2-(1-phenylvinyl)phenyl)methanol (1a) (84.1 mg, $0.40 \mathrm{mmol}), \mathrm{NBS}$ 
(2a) (178.0 mg, $1.00 \mathrm{mmol})$. Purification by column chromatography (Petroleum ether $/$ EtOAc $=100 / 1)$ yielded $\mathbf{3 a}(110.0 \mathrm{mg}, 95 \%$ yield, $84 \%$ ee $)$ as a white solid. M.p $=132-133{ }^{\circ} \mathrm{C} .[\alpha]_{\mathrm{D}}^{29}=-11.8\left(c 1.01, \mathrm{CHCl}_{3}\right)$; IR (film): 2855, 1505, 1229, $1023 \mathrm{~cm}^{-1}$; ${ }^{1} \mathrm{H}$ NMR $\left(300 \mathrm{MHz}, \mathrm{CDCl}_{3}, \mathrm{ppm}\right) \delta$ 7.58-7.52 (m, 2H), 7.40-7.22 (m, 7H), $5.31(\mathrm{~d}, J=$ $12.6 \mathrm{~Hz}, 1 \mathrm{H}), 5.20(\mathrm{~d}, J=12.6 \mathrm{~Hz}, 1 \mathrm{H}), 4.03(\mathrm{~d}, J=11.1 \mathrm{~Hz}, 1 \mathrm{H}), 3.98(\mathrm{~d}, J=11.1 \mathrm{~Hz}$, $1 \mathrm{H}) ;{ }^{13} \mathrm{C} \mathrm{NMR}\left(75 \mathrm{MHz}, \mathrm{CDCl}_{3}, \mathrm{ppm}\right) \delta 142.3,141.7,139.7,128.7,128.6,128.0,127.7$, 125.6, 122.4, 121.4, 90.1, 72.8, 40.9; HRMS (ESI) calcd. For $\mathrm{C}_{15} \mathrm{H}_{12} \mathrm{OBr}(\mathrm{M}-\mathrm{H})$ : 287.0066, Found: 287.0064.

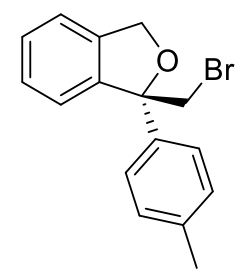

(R)-1-(Bromomethyl)-1-(p-tolyl)-1,3-dihydroisobenzofuran (3b): The Scheme 4 was followed by using (2-(1-(p-tolyl)vinyl)phenyl)methanol (1b) $(89.7 \mathrm{mg}, 0.40 \mathrm{mmol})$, NBS (2a) (178.0 mg, $1.00 \mathrm{mmol}$ ). Purification by column chromatography (Petroleum ether/EtOAc $=100 / 1)$ yielded $3 \mathbf{b}(119.4 \mathrm{mg}, 98 \%$ yield, $76 \%$ ee $)$ as colorless oil. $[\alpha]_{\mathrm{D}}^{29}$ $=-12.2\left(c\right.$ 1.05, $\left.\mathrm{CHCl}_{3}\right)$; IR (film): 2856, 1509, 1458, 1031, $728 \mathrm{~cm}^{-1} ;{ }^{1} \mathrm{H} \mathrm{NMR}(400$ $\left.\mathrm{MHz}, \mathrm{CDCl}_{3}, \mathrm{ppm}\right) \delta 7.42(\mathrm{~d}, J=8.0 \mathrm{~Hz}, 2 \mathrm{H}), 7.38-7.33(\mathrm{~m}, 1 \mathrm{H}), 7.33-7.27(\mathrm{~m}, 2 \mathrm{H})$, 7.25-7.19 (m, 1H), $7.15(\mathrm{~d}, J=8.0 \mathrm{~Hz}, 1 \mathrm{H}), 5.28(\mathrm{~d}, J=12.0 \mathrm{~Hz}, 1 \mathrm{H}), 5.18(\mathrm{~d}, J=12.0$ $\mathrm{Hz}, 1 \mathrm{H}), 4.00(\mathrm{~d}, J=11.2 \mathrm{~Hz}, 1 \mathrm{H}), 3.96(\mathrm{~d}, J=10.8 \mathrm{~Hz}, 1 \mathrm{H}), 2.31(\mathrm{~s}, 3 \mathrm{H}) ;{ }^{13} \mathrm{C} \mathrm{NMR}$ (100 MHz, $\left.\mathrm{CDCl}_{3}, \mathrm{ppm}\right) \delta 141.8,139.7,139.3,137.8,129.4,128.5,127.7,125.6,122.3$, 
121.4, 90.1, 72.8, 40.9, 21.2; HRMS (ESI) calcd. For $\mathrm{C}_{16} \mathrm{H}_{14} \mathrm{OBr}(\mathrm{M}-\mathrm{H})$ : 301.0223, Found: 301.0220.

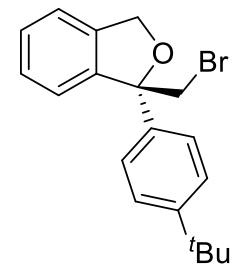

(R)-1-(Bromomethyl)-1-(4-(tert-butyl)phenyl)-1,3-dihydroisobenzofuran (3c): The Scheme 4 was followed by using (2-(1-(4-(tert-butyl)phenyl)vinyl)phenyl)methanol (1c) (106.6 mg, $0.40 \mathrm{mmol})$, NBS (2a) (178.0 mg, $1.00 \mathrm{mmol})$. Purification by column chromatography $($ Petroleum ether/EtOAc $=120 / 1)$ yielded 3c $(138.0 \mathrm{mg}, 99 \%$ yield, $78 \%$ ee) as pale yellow oil. $[\alpha]_{\mathrm{D}}^{29}=-17.2\left(c 0.82, \mathrm{CHCl}_{3}\right)$; IR (film): 2962, 1459, 1031 , $1015,746 \mathrm{~cm}^{-1} ;{ }^{1} \mathrm{H}$ NMR (400 MHz, $\left.\mathrm{CDCl}_{3}, \mathrm{ppm}\right) \delta 7.46(\mathrm{~d}, J=8.4 \mathrm{~Hz}, 2 \mathrm{H}), 7.40-7.34$ (m, 3H), 7.33-7.27 (m, 2H), 7.24-7.18 (m, 1H), $5.28(\mathrm{~d}, J=12.4 \mathrm{~Hz}, 1 \mathrm{H}), 5.17(\mathrm{~d}, J=$ $12.4 \mathrm{~Hz}, 1 \mathrm{H}), 4.03-3.95(\mathrm{~m}, 2 \mathrm{H}), 1.28(\mathrm{~s}, 9 \mathrm{H}) ;{ }^{13} \mathrm{C} \mathrm{NMR}\left(100 \mathrm{MHz}, \mathrm{CDCl}_{3}, \mathrm{ppm}\right) \delta$ $150.9,141.7,139.8,139.2,128.4,127.6,125.6,125.3,122.4,121.3,90.1,72.8,41.1$ 34.6, 31.5; HRMS (ESI) calcd. For $\mathrm{C}_{19} \mathrm{H}_{20} \mathrm{OBr}(\mathrm{M}-\mathrm{H})$ : 343.0692 , Found: 343.0688.<smiles>C=Cc1ccc(C2(CBr)OCc3ccccc32)cc1</smiles>

(R)-1-(Bromomethyl)-1-(4-vinylphenyl)-1,3-dihydroisobenzofuran $\quad$ (3d): $\quad$ The Scheme 4 was followed by using (2-(1-(4-vinylphenyl)vinyl)phenyl)methanol (1d) 
(94.5 mg, $0.40 \mathrm{mmol})$, NBS (2a) (178.0 mg, $1.00 \mathrm{mmol})$. Purification by column chromatography (Petroleum ether/EtOAc $=100 / 1)$ yielded 3d $(116.7 \mathrm{mg}, 93 \%$ yield, $77 \%$ ee) as a white solid. M.p $=93-94{ }^{\circ} \mathrm{C} .[\alpha]_{\mathrm{D}}^{24}=-12.2\left(c 0.41, \mathrm{CHCl}_{3}\right)$; IR (film): $2855,1629,1507,1459,1265,1031,909 \mathrm{~cm}^{-1} ;{ }^{1} \mathrm{H}$ NMR (400 MHz, $\left.\mathrm{CDCl}_{3}, \mathrm{ppm}\right) \delta$ $7.50(\mathrm{~d}, J=8.0 \mathrm{~Hz}, 2 \mathrm{H}), 7.44-7.27(\mathrm{~m}, 5 \mathrm{H}), 7.27-7.20(\mathrm{~m}, 1 \mathrm{H}), 6.68(\mathrm{dd}, J=17.6,10.8$ $\mathrm{Hz}, 1 \mathrm{H}), 5.73(\mathrm{~d}, J=17.6 \mathrm{~Hz}, 1 \mathrm{H}), 5.35-5.16(\mathrm{~m}, 3 \mathrm{H}), 4.01(\mathrm{~d}, J=11.2 \mathrm{~Hz}, 1 \mathrm{H}), 3.96$ $(\mathrm{d}, J=11.2 \mathrm{~Hz}, 1 \mathrm{H}) ;{ }^{13} \mathrm{C} \mathrm{NMR}\left(100 \mathrm{MHz}, \mathrm{CDCl}_{3}, \mathrm{ppm}\right) \delta 141.7,141.6,139.6,137.4$, $136.4,128.6,127.8,126.5,125.8,122.3,121.4,114.5,90.0,72.8,40.7$; HRMS (APCI) calcd. For $\mathrm{C}_{17} \mathrm{H}_{14} \mathrm{OBr}(\mathrm{M}-\mathrm{H})$ : 313.0223 , Found: 313.0222 .

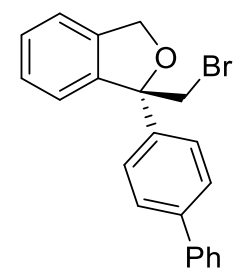

(R)-1-([1,1'-Biphenyl]-4-yl)-1-(bromomethyl)-1,3-dihydroisobenzofuran (3e): The Scheme 4 was followed by using (2-(1-([1,1'-biphenyl]-4-yl)vinyl)phenyl)methanol (1e) (114.5 mg, $0.40 \mathrm{mmol})$, NBS (2a) (178.0 mg, $1.00 \mathrm{mmol})$. Purification by column chromatography $($ Petroleum ether/EtOAc $=100 / 1)$ yielded $3 \mathbf{e}(141.1 \mathrm{mg}, 97 \%$ yield, $79 \%$ ee) as a white solid. M.p $=170-171^{\circ} \mathrm{C} .[\alpha]_{\mathrm{D}}^{24}=-14.6\left(c 0.68, \mathrm{CHCl}_{3}\right)$; IR (film): 2855, 1485, 1021, $760 \mathrm{~cm}^{-1} ;{ }^{1} \mathrm{H}$ NMR (400 MHz, $\left.\mathrm{CDCl}_{3}, \mathrm{ppm}\right) \delta$ 7.65-7.53 (m, 6H), 7.46-7.38 (m, 3H), 7.38-7.30 (m, 3H),7.29-7.22 (m, 1H), $5.32(\mathrm{~d}, J=12.4 \mathrm{~Hz}, 1 \mathrm{H})$, $5.23(\mathrm{~d}, J=12.4 \mathrm{~Hz}, 1 \mathrm{H}), 4.06(\mathrm{~d}, J=11.2 \mathrm{~Hz}, 1 \mathrm{H}), 4.01(\mathrm{~d}, J=11.2 \mathrm{~Hz}, 1 \mathrm{H}) ;{ }^{13} \mathrm{C}$ NMR (75 MHz, $\left.\mathrm{CDCl}_{3}, \mathrm{ppm}\right) \delta 141.6,141.3,141.0,140.7,139.7,129.0,128.7,127.8$, 
127.6, 127.5, 127.3, 126.1, 122.4, 121.5, 90.1, 72.9, 40.8; HRMS (APCI) calcd. For $\mathrm{C}_{21} \mathrm{H}_{16} \mathrm{OBr}(\mathrm{M}-\mathrm{H}):$ 363.0379, Found: 363.0378.

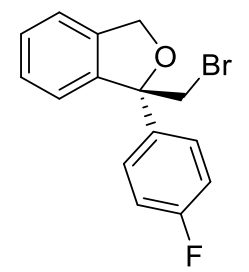

(R)-1-(Bromomethyl)-1-(4-fluorophenyl)-1,3-dihydroisobenzofuran (3f): The Scheme 4 was followed by using (2-(1-(4-fluorophenyl)vinyl)phenyl)methanol (1f) (91.3 mg, $0.40 \mathrm{mmol})$, NBS (2a) $(178.0 \mathrm{mg}, 1.00 \mathrm{mmol})$. Purification by column chromatography (Petroleum ether/EtOAc $=100 / 1)$ yielded $3 f(119.8 \mathrm{mg}, 98 \%$ yield, $80 \%$ ee) as colorless oil. $[\alpha]_{\mathrm{D}}^{29}=-12.1\left(c 1.29, \mathrm{CHCl}_{3}\right)$; IR (film): 2852, 1601, 1505, 1229, $836 \mathrm{~cm}^{-1} ;{ }^{1} \mathrm{H}$ NMR (400 MHz, $\left.\mathrm{CDCl}_{3}, \mathrm{ppm}\right) \delta$ 7.56-7.47 (m, 2H), 7.39-7.30 (m, 3H), 7.29-7.21 (m, 1H), 7.08-6.99 (m, 2H), $5.29(\mathrm{~d}, J=12.4 \mathrm{~Hz}, 1 \mathrm{H}), 5.18(\mathrm{~d}, J=12.0$ $\mathrm{Hz}, 1 \mathrm{H}), 3.98(\mathrm{~d}, J=11.2 \mathrm{~Hz}, 1 \mathrm{H}), 3.93(\mathrm{~d}, J=10.8 \mathrm{~Hz}, 1 \mathrm{H}) ;{ }^{13} \mathrm{C}$ NMR $(100 \mathrm{MHz}$ $\left.\mathrm{CDCl}_{3}, \mathrm{ppm}\right) \delta 162.5(\mathrm{~d}, J=245.0 \mathrm{~Hz}), 141.5,139.6,138.1(\mathrm{~d}, J=3.0 \mathrm{~Hz}), 128.7,127.8$, $127.6(\mathrm{~d}, J=8.0 \mathrm{~Hz}), 122.4,121.6,115.5(\mathrm{~d}, J=21.0 \mathrm{~Hz}), 89.8,72.8,40.6 ;{ }^{19} \mathrm{~F}$ NMR (376 $\left.\mathrm{MHz}, \mathrm{CDCl}_{3}, \mathrm{ppm}\right) \delta$-114.6; HRMS (ESI) calcd. For $\mathrm{C}_{15} \mathrm{H}_{11} \mathrm{OBrF}(\mathrm{M}-\mathrm{H})$ : 304.9972, Found: 304.9967.<smiles>Clc1ccc(C2(CBr)OCc3ccccc32)cc1</smiles> 
(R)-1-(Bromomethyl)-1-(4-chlorophenyl)-1,3-dihydroisobenzofuran $\quad(3 g): \quad$ The

Scheme 4 was followed by using (2-(1-(4-chlorophenyl)vinyl)phenyl)methanol (1g) (97.9 mg, $0.40 \mathrm{mmol})$, NBS (2a) $(178.0 \mathrm{mg}, 1.00 \mathrm{mmol})$. Purification by column chromatography (Petroleum ether/EtOAc $=100 / 1)$ yielded $\mathbf{3 g}(112.8 \mathrm{mg}, 87 \%$ yield, $82 \%$ ee) as colorless oil. $[\alpha]_{\mathrm{D}}^{29}=-5.7\left(c 0.83, \mathrm{CHCl}_{3}\right)$; IR (film): 2853, 1489, 1011, 758 $\mathrm{cm}^{-1} ;{ }^{1} \mathrm{H} \mathrm{NMR}\left(400 \mathrm{MHz}, \mathrm{CDCl}_{3}, \mathrm{ppm}\right) \delta 7.48(\mathrm{~d}, J=8.4 \mathrm{~Hz}, 2 \mathrm{H}), 7.37-7.29(\mathrm{~m}, 5 \mathrm{H})$, 7.27-7.21 (m, 1H), $5.29(\mathrm{~d}, J=12.4 \mathrm{~Hz}, 1 \mathrm{H}), 5.18(\mathrm{~d}, J=12.4 \mathrm{~Hz}, 1 \mathrm{H}), 3.97(\mathrm{~d}, J=$ $10.8 \mathrm{~Hz}, 1 \mathrm{H}), 3.92(\mathrm{~d}, J=10.8 \mathrm{~Hz}, 1 \mathrm{H}) ;{ }^{13} \mathrm{C} \mathrm{NMR}\left(100 \mathrm{MHz}, \mathrm{CDCl}_{3}, \mathrm{ppm}\right) \delta 141.3$, $140.8,139.5,134.0,128.8,128.7,127.9,127.2,122.3,121.6,89.7,72.8,40.4$; HRMS (ESI) calcd. For $\mathrm{C}_{15} \mathrm{H}_{11} \mathrm{OBrCl}(\mathrm{M}-\mathrm{H})$ : 320.9676, Found: 320.9670.

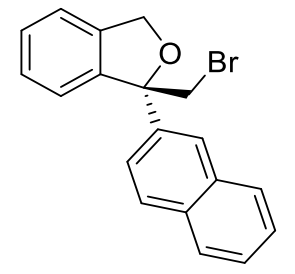

(R)-1-(Bromomethyl)-1-(naphthalen-2-yl)-1,3-dihydroisobenzofuran $\quad(3 \mathrm{~h})$ : The Scheme 4 was followed by using (2-(1-(naphthalen-2-yl)vinyl)phenyl)methanol (1h) (104.1 mg, $0.40 \mathrm{mmol})$, NBS (2a) $(178.0 \mathrm{mg}, 1.00 \mathrm{mmol})$. Purification by column chromatography (Petroleum ether/EtOAc $=100 / 1)$ yielded 3h $(134.2 \mathrm{mg}, 99 \%$ yield, $82 \%$ ee) as a white solid. M.p $=100-101{ }^{\circ} \mathrm{C} .[\alpha]_{\mathrm{D}}^{29}=-12.2\left(c 1.05, \mathrm{CHCl}_{3}\right)$; IR (film): 2855, 1507, 1459, 1030, $747 \mathrm{~cm}^{-1} ;{ }^{1} \mathrm{H} \mathrm{NMR}\left(400 \mathrm{MHz}, \mathrm{CDCl}_{3}, \mathrm{ppm}\right) \delta 8.00(\mathrm{~s}, 1 \mathrm{H})$, 7.84-7.76 (m, 3H), 7.64 (d, J=8.4 Hz, 1H), 7.49-7.42 (m, 3H), 7.38-7.31 (m, 2H), 7.29$7.23(\mathrm{~m}, 1 \mathrm{H}), 5.36(\mathrm{~d}, J=12.4 \mathrm{~Hz}, 1 \mathrm{H}), 5.27(\mathrm{~d}, J=12.4 \mathrm{~Hz}, 1 \mathrm{H}), 4.13(\mathrm{~d}, J=10.8 \mathrm{~Hz}$ 
1H), $4.07(\mathrm{~d}, J=10.8 \mathrm{~Hz}, 1 \mathrm{H}) ;{ }^{13} \mathrm{C} \mathrm{NMR}\left(100 \mathrm{MHz}, \mathrm{CDCl}_{3}, \mathrm{ppm}\right) \delta 141.7,139.8,139.6$, $133.3,133.0,128.7,128.6,128.5,127.8,127.7,126.5,126.4,124.7,123.6,122.5,121.6$ 90.3, 73.0, 40.7; HRMS (ESI) calcd. For $\mathrm{C}_{19} \mathrm{H}_{14} \mathrm{OBr}(\mathrm{M}-\mathrm{H})$ : 337.0223, Found: 337.0220 .

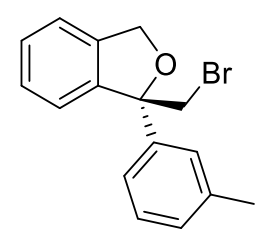

(R)-1-(Bromomethyl)-1-(m-tolyl)-1,3-dihydroisobenzofuran (3i): The Scheme 4 was followed by using (2-(1-(m-tolyl)vinyl)phenyl)methanol (1i) $(89.7 \mathrm{mg}, 0.40 \mathrm{mmol})$, NBS (2a) (178.0 mg, $1.00 \mathrm{mmol}$ ). Purification by column chromatography (Petroleum ether/EtOAc $=50 / 1)$ yielded $3 \mathbf{i}(115.1 \mathrm{mg}, 95 \%$ yield, $83 \%$ ee $)$ as colorless oil. $[\alpha]_{\mathrm{D}}^{24}=$ -17.4 (c 0.50, $\mathrm{CHCl}_{3}$ ); IR (film): 2857, 1605, 1459, 1030, $725 \mathrm{~cm}^{-1} ;{ }^{1} \mathrm{H} \mathrm{NMR}(300 \mathrm{MHz}$, $\left.\mathrm{CDCl}_{3}, \mathrm{ppm}\right) \delta 7.43-7.17(\mathrm{~m}, 7 \mathrm{H}), 7.07(\mathrm{~d}, J=7.5 \mathrm{~Hz}, 1 \mathrm{H}), 5.28(\mathrm{~d}, J=12.3 \mathrm{~Hz}, 1 \mathrm{H})$, $5.18(\mathrm{~d}, J=12.3 \mathrm{~Hz}, 1 \mathrm{H}), 4.00(\mathrm{~d}, J=10.8 \mathrm{~Hz}, 1 \mathrm{H}), 3.95(\mathrm{~d}, J=11.1 \mathrm{~Hz}, 1 \mathrm{H}), 2.33(\mathrm{~s}$, $3 \mathrm{H}) ;{ }^{13} \mathrm{C} \mathrm{NMR}\left(75 \mathrm{MHz}, \mathrm{CDCl}_{3}, \mathrm{ppm}\right) \delta 142.2,141.7,139.6,138.3,128.8,128.5,128.4$, 127.7, 126.3, 122.6, 122.4, 121.4, 90.1, 72.8, 41.0, 21.8; HRMS (APCI) calcd. For $\mathrm{C}_{16} \mathrm{H}_{14} \mathrm{OBr}(\mathrm{M}-\mathrm{H}): 301.0223$, Found: 301.0224.

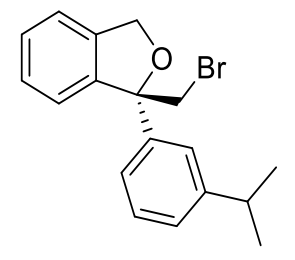


(R)-1-(Bromomethyl)-1-(3-isopropylphenyl)-1,3-dihydroisobenzofuran (3j): The

Scheme 4 was followed by using (2-(1-(3-isopropylphenyl)vinyl)phenyl)methanol (1j) (100.9 mg, $0.40 \mathrm{mmol})$, NBS (2a) $(178.0 \mathrm{mg}, 1.00 \mathrm{mmol})$. Purification by column chromatography (Petroleum ether/EtOAc $=100 / 1)$ yielded 3j $(126.2 \mathrm{mg}, 95 \%$ yield, $81 \%$ ee) as colorless oil. $[\alpha]_{\mathrm{D}}^{25}=-14.3\left(c 0.90, \mathrm{CHCl}_{3}\right)$; IR (film): 2960, 1604, 1462 , 1030, $707 \mathrm{~cm}^{-1} ;{ }^{1} \mathrm{H}$ NMR (300 MHz, $\left.\mathrm{CDCl}_{3}, \mathrm{ppm}\right) \delta 7.43-7.18(\mathrm{~m}, 7 \mathrm{H}), 7.14(\mathrm{~d}, J=7.5$ Hz, 1H), 5.29 (d, $J=12.0 \mathrm{~Hz}, 1 \mathrm{H}), 5.19(\mathrm{~d}, J=12.0 \mathrm{~Hz}, 1 \mathrm{H}), 4.05-3.94$ (m, 2H), 2.90 (hept, $J=6.9 \mathrm{~Hz}, 1 \mathrm{H}), 1.23(\mathrm{~d}, J=6.9 \mathrm{~Hz}, 6 \mathrm{H}) ;{ }^{13} \mathrm{C} \mathrm{NMR}\left(100 \mathrm{MHz}, \mathrm{CDCl}_{3}, \mathrm{ppm}\right) \delta$ $149.3,142.1,141.8,139.7,128.6,128.5,127.7,126.0,123.8,123.0,122.4,121.4,90.3$, 72.9, 41.2, 34.4, 24.1; HRMS (APCI) calcd. For $\mathrm{C}_{18} \mathrm{H}_{18} \mathrm{OBr}(\mathrm{M}-\mathrm{H})$ : 329.0536, Found: 329.0534 .

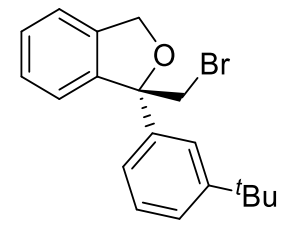

(R)-1-(Bromomethyl)-1-(3-(tert-butyl)phenyl)-1,3-dihydroisobenzofuran (3k): The Scheme 4 was followed by using (2-(1-(3-(tert-butyl)phenyl)vinyl)phenyl)methanol (1k) (106.6 mg, $0.40 \mathrm{mmol})$, NBS (2a) (178.0 mg, $1.00 \mathrm{mmol})$. Purification by column chromatography (Petroleum ether/EtOAc $=100 / 1)$ yielded 3k $(135.1 \mathrm{mg}, 98 \%$ yield, $74 \%$ ee) as colorless oil. $[\alpha]_{\mathrm{D}}^{24}=-22.4\left(c 0.71, \mathrm{CHCl}_{3}\right)$; IR (film): 2962, 1601, 1460, 1032, $761 \mathrm{~cm}^{-1} ;{ }^{1} \mathrm{H}$ NMR $\left(300 \mathrm{MHz}, \mathrm{CDCl}_{3}, \mathrm{ppm}\right) \delta$ 7.63-7.59 (m, 1H), 7.43-7.21 (m, $7 \mathrm{H}), 5.31(\mathrm{~d}, J=12.3 \mathrm{~Hz}, 1 \mathrm{H}), 5.20(\mathrm{~d}, J=12.0 \mathrm{~Hz}, 1 \mathrm{H}), 4.01(\mathrm{~s}, 2 \mathrm{H}), 1.31(\mathrm{~s}, 9 \mathrm{H}) ;{ }^{13} \mathrm{C}$ NMR (75 MHz, $\left.\mathrm{CDCl}_{3}, \mathrm{ppm}\right) \delta 151.6,141.8,139.8,128.5,128.3,127.7,125.0,122.7$, 
122.5, 122.4, 121.4, 90.4, 72.8, 41.3, 35.0, 31.5; HRMS (APCI) calcd. For $\mathrm{C}_{19} \mathrm{H}_{20} \mathrm{OBr}$ (M-H): 343.0692, Found: 343.0691.

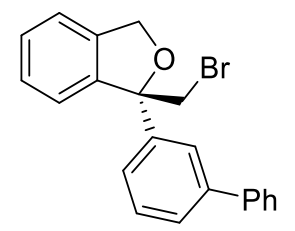

(R)-1-([1,1'-Biphenyl]-3-yl)-1-(bromomethyl)-1,3-dihydroisobenzofuran (3I): The Scheme 4 was followed by using (2-(1-([1,1'-biphenyl]-3-yl)vinyl)phenyl)methanol (11) (114.5 mg, $0.40 \mathrm{mmol})$, NBS (2a) $(178.0 \mathrm{mg}, 1.00 \mathrm{mmol})$. Purification by column chromatography (Petroleum ether/EtOAc $=100 / 1)$ yielded $31(139.0 \mathrm{mg}, 95 \%$ yield, 77\% ee) as colorless oil. $[\alpha]_{\mathrm{D}}^{24}=-15.7\left(c 0.35, \mathrm{CHCl}_{3}\right)$; IR (film): 2857, 1598, 1478, 1031, $778 \mathrm{~cm}^{-1} ;{ }^{1} \mathrm{H}$ NMR $\left(300 \mathrm{MHz}, \mathrm{CDCl}_{3}, \mathrm{ppm}\right) \delta 7.78(\mathrm{~s}, 1 \mathrm{H}), 7.61-7.29(\mathrm{~m}, 11 \mathrm{H}), 7.29$ $7.21(\mathrm{~m}, 1 \mathrm{H}), 5.33(\mathrm{~d}, J=12.3 \mathrm{~Hz}, 1 \mathrm{H}), 5.24(\mathrm{~d}, J=12.3 \mathrm{~Hz}, 1 \mathrm{H}), 4.07(\mathrm{~d}, J=11.1 \mathrm{~Hz}$, 1H), $4.02(\mathrm{~d}, J=11.1 \mathrm{~Hz}, 1 \mathrm{H}) ;{ }^{13} \mathrm{C} \mathrm{NMR}\left(75 \mathrm{MHz}, \mathrm{CDCl}_{3}, \mathrm{ppm}\right) \delta 142.8,141.7,141.6$, $141.2,139.7,129.1,129.0,128.6,127.8,127.6,127.4,126.9,124.5,122.4,121.5,90.2$ 72.9, 40.9; HRMS (APCI) calcd. For $\mathrm{C}_{21} \mathrm{H}_{16} \mathrm{OBr}(\mathrm{M}-\mathrm{H})$ : 363.0379, Found: 363.0378.

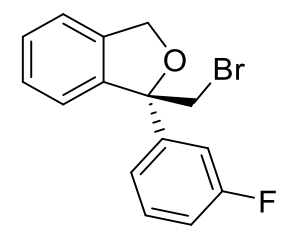

(R)-1-(Bromomethyl)-1-(3-fluorophenyl)-1,3-dihydroisobenzofuran (3m): The Scheme 4 was followed by using (2-(1-(3-fluorophenyl)vinyl)phenyl)methanol (1m) (91.3 mg, $0.40 \mathrm{mmol})$, NBS (2a) $(178.0 \mathrm{mg}, 1.00 \mathrm{mmol})$. Purification by column 
chromatography (Petroleum ether/EtOAc $=60 / 1)$ yielded 3m $(112.2 \mathrm{mg}, 91 \%$ yield, $86 \%$ ee) as pale yellow oil. $[\alpha]_{\mathrm{D}}^{24}=-1.8\left(c 0.72, \mathrm{CHCl}_{3}\right)$; IR (film): $2858,1588,1484$, 1266, $726 \mathrm{~cm}^{-1} ;{ }^{1} \mathrm{H}$ NMR (300 MHz, $\left.\mathrm{CDCl}_{3}, \mathrm{ppm}\right)$ 7.44-7.21 (m, 7H), 7.03-6.91 (m, 1H), $5.31(\mathrm{~d}, J=12.3 \mathrm{~Hz}, 1 \mathrm{H}), 5.21(\mathrm{~d}, J=12.3 \mathrm{~Hz}, 1 \mathrm{H}), 3.99(\mathrm{~d}, J=11.1 \mathrm{~Hz}, 1 \mathrm{H})$, $3.94(\mathrm{~d}, J=10.8 \mathrm{~Hz}, 1 \mathrm{H}) ;{ }^{13} \mathrm{C} \mathrm{NMR}\left(75 \mathrm{MHz}, \mathrm{CDCl}_{3}, \mathrm{ppm}\right) \delta 163.1(\mathrm{~d}, J=245.3 \mathrm{~Hz})$, $145.1,141.2,139.6,130.2(\mathrm{~d}, J=8.3 \mathrm{~Hz}), 128.8,127.9,122.3,121.6,121.1(\mathrm{~d}, J=3.0$ $\mathrm{Hz}), 114.9(\mathrm{~d}, J=21.0 \mathrm{~Hz}), 113.1(\mathrm{~d}, J=23.3 \mathrm{~Hz}), 89.7,72.9,40.4 ;{ }^{19} \mathrm{~F}$ NMR $(376$ $\left.\mathrm{MHz}, \mathrm{CDCl}_{3}, \mathrm{ppm}\right) \delta$-112.7; HRMS (APCI) calcd. For $\mathrm{C}_{15} \mathrm{H}_{11} \mathrm{OBrF}(\mathrm{M}-\mathrm{H}): 304.9972$, Found: 304.9972.

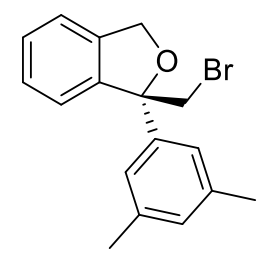

(R)-1-(Bromomethyl)-1-(3,5-dimethylphenyl)-1,3-dihydroisobenzofuran (3n): The Scheme 4 was followed by using (2-(1-(3,5-dimethylphenyl)vinyl)phenyl)methanol (1n) $(95.3 \mathrm{mg}, 0.40 \mathrm{mmol})$, NBS (2a) (178.0 mg, $1.00 \mathrm{mmol})$. Purification by column chromatography (Petroleum ether/EtOAc $=50 / 1)$ yielded 3n $(116.0 \mathrm{mg}, 92 \%$ yield, $84 \%$ ee) as colorless oil. $[\alpha]_{\mathrm{D}}^{24}=-13.1\left(c 0.35, \mathrm{CHCl}_{3}\right)$; IR (film): $2915,1604,1461,1039$ $\mathrm{cm}^{-1} ;{ }^{1} \mathrm{H}$ NMR $\left(300 \mathrm{MHz}, \mathrm{CDCl}_{3}, \mathrm{ppm}\right) \delta$ 7.33-7.15 (m, 3H), 7.15-7.00 (m, 3H), 6.81 (s, 1H), $5.18(\mathrm{~d}, J=12.3 \mathrm{~Hz}, 1 \mathrm{H}), 5.09(\mathrm{~d}, J=12.3 \mathrm{~Hz}, 1 \mathrm{H}), 3.90(\mathrm{~d}, J=11.1 \mathrm{~Hz}, 1 \mathrm{H})$, $3.85(\mathrm{~d}, J=11.1 \mathrm{~Hz}, 1 \mathrm{H}), 2.20(\mathrm{~s}, 6 \mathrm{H}) ;{ }^{13} \mathrm{C} \mathrm{NMR}\left(75 \mathrm{MHz}, \mathrm{CDCl}_{3}, \mathrm{ppm}\right) \delta 142.2,141.7$, 139.6, 138.2, 129.7, 128.4, 127.6, 123.3, 122.4, 121.3, 90.1, 72.8, 41.1, 21.7; HRMS (APCI) calcd. For $\mathrm{C}_{17} \mathrm{H}_{16} \mathrm{OBr}(\mathrm{M}-\mathrm{H}): 315.0379$, Found: 315.0378. 


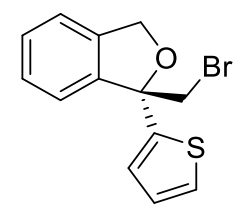

(S)-1-(Bromomethyl)-1-(thiophen-2-yl)-1,3-dihydroisobenzofuran $\quad$ (3o): The

Scheme 4 was followed by using (2-(1-(thiophen-2-yl)vinyl)phenyl)methanol (10) (86.5 mg, $0.40 \mathrm{mmol})$, NBS (2a) $(178.0 \mathrm{mg}, 1.00 \mathrm{mmol})$. Purification by column chromatography (Petroleum ether/EtOAc $=100 / 1)$ yielded $30(117.0 \mathrm{mg}, 99 \%$ yield, $53 \%$ ee) as pale yellow oil. $[\alpha]_{\mathrm{D}}^{29}=-37.6\left(c 1.00, \mathrm{CHCl}_{3}\right)$; IR (film): 2860, 1458, 1018, $705 \mathrm{~cm}^{-1} ;{ }^{1} \mathrm{H}$ NMR $\left(400 \mathrm{MHz}, \mathrm{CDCl}_{3}, \mathrm{ppm}\right) \delta$ 7.41-7.32 (m, 3H), 7.28-7.22 (m, 2H), 7.04-7.01 (m, 1H), 7.00-6.95 (m, 1H), $5.30(\mathrm{~d}, J=12.4 \mathrm{~Hz}, 1 \mathrm{H}), 5.24(\mathrm{~d}, J=12.0 \mathrm{~Hz}$, 1H), $4.05(\mathrm{~d}, J=10.8 \mathrm{~Hz}, 1 \mathrm{H}), 3.99(\mathrm{~d}, J=10.8 \mathrm{~Hz}, 1 \mathrm{H}) ;{ }^{13} \mathrm{C} \mathrm{NMR}\left(100 \mathrm{MHz}, \mathrm{CDCl}_{3}\right.$, ppm) $\delta 146.9,141.2,139.5,128.9,127.9,127.2,125.5,123.9,122.1,121.4,88.7,73.4$, 40.9; HRMS (ESI) calcd. For $\mathrm{C}_{13} \mathrm{H}_{10} \mathrm{OBrS}(\mathrm{M}-\mathrm{H})$ : 292.9630, Found: 292.9625.

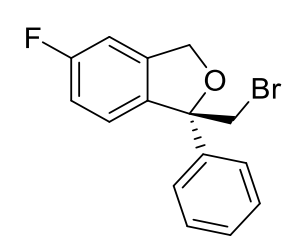

(R)-1-(Bromomethyl)-5-fluoro-1-phenyl-1,3-dihydroisobenzofuran $\quad$ (3p): $\quad$ The Scheme 4 was followed by using (5-fluoro-2-(1-phenylvinyl)phenyl)methanol (1p) (91.3 mg, $0.40 \mathrm{mmol})$, NBS (2a) (178.0 mg, $1.00 \mathrm{mmol})$. Purification by column chromatography $($ Petroleum ether/EtOAc $=100 / 1)$ yielded 3p $(99.1 \mathrm{mg}, 81 \%$ yield, $75 \%$ ee) as colorless oil. $[\alpha]_{\mathrm{D}}^{29}=-16.5\left(c 1.02, \mathrm{CHCl}_{3}\right)$; IR (film): 2854, 1603, 1486, 1264, $1027 \mathrm{~cm}^{-1} ;{ }^{1} \mathrm{H}$ NMR $\left(400 \mathrm{MHz}, \mathrm{CDCl}_{3}, \mathrm{ppm}\right) \delta 7.51(\mathrm{~d}, J=7.6 \mathrm{~Hz}, 2 \mathrm{H}), 7.41-7.27(\mathrm{~m}$, 
4H), 7.07-6.98 (m, 1H), $6.93(\mathrm{~d}, J=8.4 \mathrm{~Hz}, 1 \mathrm{H}), 5.27(\mathrm{~d}, J=12.8 \mathrm{~Hz}, 1 \mathrm{H}), 5.15(\mathrm{~d}, J$ $=12.4 \mathrm{~Hz}, 1 \mathrm{H}), 3.99(\mathrm{~d}, J=11.2 \mathrm{~Hz}, 1 \mathrm{H}), 3.96(\mathrm{~d}, J=11.6 \mathrm{~Hz}, 1 \mathrm{H}) ;{ }^{13} \mathrm{C} \mathrm{NMR}(100$ $\left.\mathrm{MHz}, \mathrm{CDCl}_{3}, \mathrm{ppm}\right) \delta 163.3(\mathrm{~d}, J=245.0 \mathrm{~Hz}), 142.1(\mathrm{~d}, J=9.0 \mathrm{~Hz}), 142.0,137.3,128.8$, $128.2,125.6,123.8(\mathrm{~d}, J=9.0 \mathrm{~Hz}), 115.1(\mathrm{~d}, J=23.0 \mathrm{~Hz}), 108.8(\mathrm{~d}, J=24.0 \mathrm{~Hz}), 89.9$, $72.4(\mathrm{~d}, J=2.0 \mathrm{~Hz}), 40.8 ;{ }^{19} \mathrm{~F}$ NMR $\left(376 \mathrm{MHz}, \mathrm{CDCl}_{3}, \mathrm{ppm}\right) \delta-114.0 ;$ HRMS (ESI) calcd. For $\mathrm{C}_{15} \mathrm{H}_{11} \mathrm{OBrF}(\mathrm{M}-\mathrm{H})$ : 304.9972 , Found: 304.9967.<smiles>N#Cc1ccc2c(c1)COC2(Br)c1ccc(F)cc1</smiles>

(R)-1-(Bromomethyl)-1-(4-fluorophenyl)-1,3-dihydroisobenzofuran-5-carbonitrile (3q): The Scheme 4 was followed by using 4-(1-(4-Fluorophenyl)vinyl)-3(hydroxymethyl)benzonitrile (1q) (101.3 mg, 0.40 mmol), NBS (2a) (178.0 mg, 1.00 mmol). Purification by column chromatography $($ Petroleum ether/EtOAc $=10 / 1)$ yielded 3q (129.0 mg, 97\% yield, 78\% ee) as pale yellow oil. $[\alpha]_{\mathrm{D}}^{24}=-22.0(c 0.44$, $\mathrm{CHCl}_{3}$ ); IR (film): 2921, 1601, 1507, 1227, $834 \mathrm{~cm}^{-1} ;{ }^{1} \mathrm{H} \mathrm{NMR}\left(400 \mathrm{MHz}, \mathrm{CDCl}_{3}, \mathrm{ppm}\right)$ $\delta 7.69(\mathrm{~d}, J=7.6 \mathrm{~Hz}, 1 \mathrm{H}), 7.58(\mathrm{~s}, 1 \mathrm{H}), 7.54-7.44(\mathrm{~m}, 3 \mathrm{H}), 7.13-7.04(\mathrm{~m}, 2 \mathrm{H}), 5.34(\mathrm{~d}$, $J=12.8 \mathrm{~Hz}, 1 \mathrm{H}), 5.20(\mathrm{~d}, J=12.8 \mathrm{~Hz}, 1 \mathrm{H}), 3.99(\mathrm{~d}, J=11.2 \mathrm{~Hz}, 1 \mathrm{H}), 3.96(\mathrm{~d}, J=11.2$ $\mathrm{Hz}, 1 \mathrm{H}) ;{ }^{13} \mathrm{C} \mathrm{NMR}\left(100 \mathrm{MHz}, \mathrm{CDCl}_{3}, \mathrm{ppm}\right) \delta 162.8(\mathrm{~d}, J=249.0 \mathrm{~Hz}), 146.5,141.1$, $136.7(\mathrm{~d}, J=3.0 \mathrm{~Hz}), 132.1,127.4(\mathrm{~d}, J=8.0 \mathrm{~Hz}), 125.6,123.4,118.6,115.9(\mathrm{~d}, J=$ 21.0 Hz), 112.9, 90.1, 72.3, 39.6; ${ }^{19} \mathrm{~F}$ NMR (376 MHz, $\left.\mathrm{CDCl}_{3}, \mathrm{ppm}\right) \delta$-113.4; HRMS (EI) calcd. For $\mathrm{C}_{16} \mathrm{H}_{10} \mathrm{FNO}$ (M-H-Br): 251.0741, Found: 251.0743. 
<smiles>Fc1ccc2c(c1)C(CBr)(c1ccccc1)OC2</smiles>

(R)-1-(Bromomethyl)-6-fluoro-1-phenyl-1,3-dihydroisobenzofuran $\quad$ (3r): The Scheme 4 was followed by using (4-fluoro-2-(1-phenylvinyl)phenyl)methanol (1r) (91.3 mg, $0.40 \mathrm{mmol})$, NBS (2a) $(178.0 \mathrm{mg}, 1.00 \mathrm{mmol})$. Purification by column chromatography $($ Petroleum ether/EtOAc $=100 / 1)$ yielded 3e $(108.5 \mathrm{mg}, 88 \%$ yield, $83 \%$ ee) as a white solid. M.p $=123-125^{\circ} \mathrm{C} .[\alpha]_{\mathrm{D}}^{29}=-8.4\left(c 0.5, \mathrm{CHCl}_{3}\right)$; IR (film): 2868, 1603, 1490, $1059 \mathrm{~cm}^{-1} ;{ }^{1} \mathrm{H}$ NMR $\left(400 \mathrm{MHz}, \mathrm{CDCl}_{3}, \mathrm{ppm}\right) \delta 7.52(\mathrm{~d}, J=7.6 \mathrm{~Hz}$, 2H), 7.41-7.34 (m, 2H), 7.33-7.26 (m, 1H), 7.21-7.15 (m, 2H), 7.11-6.98 (m, 2H),5.27 $(\mathrm{d}, J=12.0 \mathrm{~Hz}, 1 \mathrm{H}), 5.16(\mathrm{~d}, J=12.0 \mathrm{~Hz}, 1 \mathrm{H}), 3.97(\mathrm{~s}, 2 \mathrm{H}) ;{ }^{13} \mathrm{C}$ NMR $(100 \mathrm{MHz}$, $\left.\mathrm{CDCl}_{3}, \mathrm{ppm}\right) \delta 162.8(\mathrm{~d}, J=244.0 \mathrm{~Hz}), 143.9(\mathrm{~d}, J=7.0 \mathrm{~Hz}), 141.7,135.1(\mathrm{~d}, J=2.0$ $\mathrm{Hz}), 128.9,128.3,125.6,122.7(\mathrm{~d}, J=9.0 \mathrm{~Hz}), 115.9$ (d, $J=23.0 \mathrm{~Hz}), 109.8(\mathrm{~d}, J=$ 24.0 Hz), $90.1(\mathrm{~d}, J=2.0 \mathrm{~Hz}), 72.6,40.4 ;{ }^{19} \mathrm{~F} \mathrm{NMR}\left(376 \mathrm{MHz}, \mathrm{CDCl}_{3}, \mathrm{ppm}\right) \delta-114.8$; HRMS (ESI) calcd. For $\mathrm{C}_{15} \mathrm{H}_{11} \mathrm{OBrF}(\mathrm{M}-\mathrm{H})$ : 304.9972, Found: 304.9967.<smiles>ICC1(c2ccccc2)OCc2ccccc21</smiles>

(R)-1-(iodomethyl)-1-phenyl-1,3-dihydroisobenzofuran (3s): The Scheme 4 was followed by using (2-(1-phenylvinyl)phenyl)methanol (1a) (84.1 mg, $0.40 \mathrm{mmol})$, NIS (2d) $(225.0 \mathrm{mg}, 1.00 \mathrm{mmol})$. Purification by column chromatography (Petroleum ether/EtOAc $=100 / 1)$ yielded 3s $(126.4 \mathrm{mg}, 94 \%$ yield, 69\% ee $)$ as a yellow solid. M.p 
$=80-81^{\circ} \mathrm{C} .[\alpha]_{\mathrm{D}}^{24}=+14.4\left(c 0.52, \mathrm{CHCl}_{3}\right)$; IR (film): 2850, 1458, 1265, $739 \mathrm{~cm}^{-1} ;{ }^{1} \mathrm{H}$ NMR (300 MHz, $\left.\mathrm{CDCl}_{3}, \mathrm{ppm}\right) \delta$ 7.60-7.53 (m, 2H), 7.42-7.22 (m, 7H), $5.32(\mathrm{~d}, J=$ $12.3 \mathrm{~Hz}, 1 \mathrm{H}), 5.21(\mathrm{~d}, J=12.6 \mathrm{~Hz}, 1 \mathrm{H}), 3.94(\mathrm{~d}, J=10.8 \mathrm{~Hz}, 1 \mathrm{H}), 3.89(\mathrm{~d}, J=11.1 \mathrm{~Hz}$, $1 \mathrm{H}) ;{ }^{13} \mathrm{C}$ NMR $\left(100 \mathrm{MHz}, \mathrm{CDCl}_{3}, \mathrm{ppm}\right) \delta 142.4,142.3,139.5,128.7,128.6,128.0$, 127.8, 125.4, 122.1, 121.5, 89.7, 72.7, 17.5; HRMS (APCI) calcd. For $\mathrm{C}_{15} \mathrm{H}_{12} \mathrm{OI}$ (MH): 334.9927, Found: 334.9928.

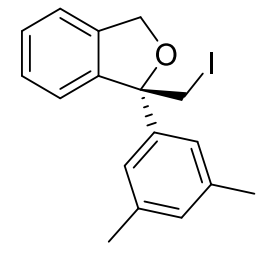

(R)-1-(3,5-Dimethylphenyl)-1-(iodomethyl)-1,3-dihydroisobenzofuran (3t): The Scheme 4 was followed by using (2-(1-(3,5-dimethylphenyl)vinyl)phenyl)methanol (1n) $(95.3 \mathrm{mg}, 0.40 \mathrm{mmol})$, NIS (2d) $(225.0 \mathrm{mg}, 1.00 \mathrm{mmol})$. Purification by column chromatography $($ Petroleum ether/EtOAc $=100 / 1)$ yielded 3t $(132.1 \mathrm{mg}, 91 \%$ yield, $59 \%$ ee) as yellow oil. $[\alpha]_{\mathrm{D}}^{24}=+12.3\left(c 0.43, \mathrm{CHCl}_{3}\right)$; IR (film): 2913, 1606, 1462, 1265 , $726 \mathrm{~cm}^{-1} ;{ }^{1} \mathrm{H}$ NMR $\left(400 \mathrm{MHz}, \mathrm{CDCl}_{3}, \mathrm{ppm}\right) \delta$ 7.38-7.29 (m, 3H), 7.29-7.18 (m, 1H), 7.17-7.11 (m, 2H), $6.90(\mathrm{~s}, 1 \mathrm{H}), 5.28$ (d, $J=12.0 \mathrm{~Hz}, 1 \mathrm{H}), 5.19(\mathrm{~d}, J=12.0 \mathrm{~Hz}, 1 \mathrm{H})$, 3.93-3.81 (m, 2H), $2.30(\mathrm{~s}, 6 \mathrm{H}) ;{ }^{13} \mathrm{C} \mathrm{NMR}\left(100 \mathrm{MHz}, \mathrm{CDCl}_{3}, \mathrm{ppm}\right) \delta 142.5,142.3$, $139.5,138.3,129.7,128.5,127.8,123.2,122.2,121.4,89.8,72.7,21.7,17.9$; HRMS (APCI) calcd. For $\mathrm{C}_{17} \mathrm{H}_{16} \mathrm{OI}(\mathrm{M}-\mathrm{H}): 363.0240$, Found: 363.0241.

\section{Transformations compound 3a (Scheme 6)}


To a solution of $3 \mathbf{a}(0.0578 \mathrm{~g}, 0.20 \mathrm{mmol})$ in $\mathrm{CH}_{3} \mathrm{CN}(1.5 \mathrm{~mL})$ was added $\mathrm{K}_{2} \mathrm{CO}_{3}$ (0.0553 g, $0.40 \mathrm{mmol})$ and 4-chlorobenzenethiol (0.0868 g, $0.60 \mathrm{mmol})$. The reaction mixture was warmed to $80{ }^{\circ} \mathrm{C}$ and stirred for $24 \mathrm{~h}$. After cooling to room temperature, the solvent was removed under reduced pressure. The residue was purified by flash column chromatography on silica gel (petroleum ether/ethyl acetate $=10 / 1$ ) to give product 7 .<smiles>Clc1ccc(SC[C@]2(c3ccccc3)OCc3ccccc32)cc1</smiles>

(R)-1-(((4-chlorophenyl)thio)methyl)-1-phenyl-1,3-dihydroisobenzofuran

colorless oil, $68.1 \mathrm{mg}, 96 \%$ yield, $98 \%$ ee, $[\alpha]_{\mathrm{D}}^{24}=+23.5\left(c 0.34, \mathrm{CHCl}_{3}\right)$; IR (film): 2920, 1650, 1475, 1094, $814 \mathrm{~cm}^{-1} ;{ }^{1} \mathrm{H}$ NMR (400 MHz, $\left.\mathrm{CDCl}_{3}, \mathrm{ppm}\right) \delta 7.53$ (d, $J=7.6$ Hz, 2H), 7.39-7.07 (m, 11H), $5.25(\mathrm{~d}, J=12.0 \mathrm{~Hz}, 1 \mathrm{H}), 5.18(\mathrm{~d}, J=12.4 \mathrm{~Hz}, 1 \mathrm{H}), 3.76$ $(\mathrm{d}, J=13.6 \mathrm{~Hz}, 1 \mathrm{H}), 3.68(\mathrm{~d}, J=13.2 \mathrm{~Hz}, 1 \mathrm{H}) ;{ }^{13} \mathrm{C} \mathrm{NMR}\left(100 \mathrm{MHz}, \mathrm{CDCl}_{3}, \mathrm{ppm}\right) \delta$ $143.8,142.5,139.6,136.2,132.1,131.3,128.9,128.6,128.3,127.7,127.6,125.4,122.4$ 121.3, 90.9, 72.8, 47.2; HRMS (ESI) calcd. For $\mathrm{C}_{21} \mathrm{H}_{16} \mathrm{OClS}(\mathrm{M}-\mathrm{H}): 351.0605$, Found: 351.0605 .

To a stainless-steel autoclave were added 3a $(0.0578 \mathrm{~g}, 0.20 \mathrm{mmol})$, dry $\mathrm{MeOH}$ $(1.0 \mathrm{~mL}), \mathrm{Et}_{3} \mathrm{~N}(0.028 \mathrm{~mL}, 0.20 \mathrm{mmol})$ and $5 \% \mathrm{Pd} / \mathrm{C}(0.0852 \mathrm{~g}, 0.04 \mathrm{mmol})$ in the nitrogen atmosphere glovebox. After being sealed, the autoclave was purged three times with $\mathrm{H}_{2}$ and the final pressure of hydrogen was adjusted to 20 bar. The reaction mixture was stirred at $30{ }^{\circ} \mathrm{C}$ for $12 \mathrm{~h}$. Then the solvent was removed under reduced pressure. 
The crude residue was purified by flash column chromatography on silica gel (petroleum ether/ethyl acetate $=100 / 1)$ to give product $\mathbf{8}$.<smiles>CC1(c2ccccc2)OCc2ccccc21</smiles>

(R)-1-Methyl-1-phenyl-1,3-dihydroisobenzofuran (8): colorless oil, $38.4 \mathrm{mg}, 91 \%$ yield, $98 \%$ ee, $[\alpha]_{\mathrm{D}}^{24}=+41.9\left(c 0.11, \mathrm{CHCl}_{3}\right) ;{ }^{1} \mathrm{H} \mathrm{NMR}\left(400 \mathrm{MHz}, \mathrm{CDCl}_{3}, \mathrm{ppm}\right) \delta 7.48$ $(\mathrm{d}, J=7.6 \mathrm{~Hz}, 2 \mathrm{H}), 7.33-7.17(\mathrm{~m}, 7 \mathrm{H}), 5.18(\mathrm{~d}, J=12.0 \mathrm{~Hz}, 1 \mathrm{H}), 5.13(\mathrm{~d}, J=12.4 \mathrm{~Hz}$, 1H), $1.87(\mathrm{~s}, 3 \mathrm{H}) ;{ }^{13} \mathrm{C}$ NMR (100 MHz, $\left.\mathrm{CDCl}_{3}, \mathrm{ppm}\right) \delta 146.0,145.9,139.0,128.4$, $127.6,127.5,127.2,125.4,122.1,121.3,88.8,71.6,28.7$.

Zhang, L.; Zhang, W.; Liu, J.; Hu, J. J. Org. Chem. 2009, 74, 2850-2853.

3a $(0.0578 \mathrm{~g}, 0.20 \mathrm{mmol})$ was dissolved in DCM (2.0 mL), and NBS (0.1424 g, $0.8 \mathrm{mmol}$ ) was added. The reaction mixture was stirred overnight. The solvent was removed and the crude residue was purified by flash column chromatography on silica gel (petroleum ether/ethyl acetate $=10 / 1)$ to give product 9a.<smiles>O=C1OC(CBr)(c2ccccc2)c2ccccc21</smiles>

(R)-3-(Bromomethyl)-3-phenylisobenzofuran-1(3H)-one (9a): white solid, $57.7 \mathrm{mg}$, $95 \%$ yield, $97 \%$ ee, $[\alpha]_{\mathrm{D}}^{25}=-7.6\left(c 0.49, \mathrm{CHCl}_{3}\right) ;{ }^{1} \mathrm{H} \mathrm{NMR}\left(300 \mathrm{MHz}, \mathrm{CDCl}_{3}, \mathrm{ppm}\right) \delta$ $7.96(\mathrm{~d}, J=7.5 \mathrm{~Hz}, 1 \mathrm{H}), 7.81-7.73(\mathrm{~m}, 1 \mathrm{H}), 7.72-7.66(\mathrm{~m}, 1 \mathrm{H}), 7.66-7.56(\mathrm{~m}, 3 \mathrm{H}), 7.48-$ $7.35(\mathrm{~m}, 3 \mathrm{H}), 4.17(\mathrm{~d}, J=11.4 \mathrm{~Hz}, 1 \mathrm{H}), 4.11(\mathrm{~d}, J=11.4 \mathrm{~Hz}, 1 \mathrm{H}) ;{ }^{13} \mathrm{C} \mathrm{NMR}(100 \mathrm{MHz}$ 
$\left.\mathrm{CDCl}_{3}, \mathrm{ppm}\right) \delta 169.1,150.0,137.7,134.6,130.2,129.4,129.2,126.8,126.2,125.7$, $122.8,87.5,38.1$.

Okada, M.; Kaneko, K.; Yamanaka, M.; Shirakawa, S. Org. Biomol. Chem. 2019, 17, $3747-3751$.<smiles>O=C1OC(CBr)(c2ccc(Br)cc2)c2ccccc21</smiles>

(R)-3-(Bromomethyl)-3-(4-(tert-butyl)phenyl)isobenzofuran-1(3H)-one (9b): The titled compound was followed by using (R)-1-(bromomethyl)-1-(4-(tert-butyl)phenyl)1,3-dihydroisobenzofuran (3c) (69.1 mg, $0.20 \mathrm{mmol})$, NBS (2a) (142.4 mg, $0.8 \mathrm{mmol})$. Purification by column chromatography $($ Petroleum ether/EtOAc $=40 / 1)$ yielded $\mathbf{9 b}$ (67.4 mg, 94\% yield, $77 \%$ ee) as a white solid. M.p $=174-175^{\circ} \mathrm{C} .[\alpha]_{\mathrm{D}}^{31}=-4.53(c 0.64$, $\mathrm{CHCl}_{3}$ ); IR (film): 2963, 1771, 1084, $761 \mathrm{~cm}^{-1} ;{ }^{1} \mathrm{H} \mathrm{NMR}\left(400 \mathrm{MHz}, \mathrm{CDCl}_{3}, \mathrm{ppm}\right) \delta$ $7.93(\mathrm{~d}, J=7.6 \mathrm{~Hz}, 1 \mathrm{H}), 7.77-7.70(\mathrm{~m}, 1 \mathrm{H}), 7.66(\mathrm{~d}, J=7.6 \mathrm{~Hz}, 1 \mathrm{H}), 7.62-7.54(\mathrm{~m}, 1 \mathrm{H})$, $7.49(\mathrm{~d}, J=8.4 \mathrm{~Hz}, 2 \mathrm{H}), 7.41(\mathrm{~d}, J=7.4 \mathrm{~Hz}, 2 \mathrm{H}), 4.15(\mathrm{~d}, J=11.2 \mathrm{~Hz}, 1 \mathrm{H}), 4.09(\mathrm{~d}, J$ $=11.2 \mathrm{~Hz}, 1 \mathrm{H}), 1.30(\mathrm{~s}, 9 \mathrm{H}) ;{ }^{13} \mathrm{C} \mathrm{NMR}\left(100 \mathrm{MHz}, \mathrm{CDCl}_{3}, \mathrm{ppm}\right) \delta 169.2,152.5,150.1$, 134.6, 134.5, 130.1, 128.8, 126.9, 126.1, 125.5, 122.8, 87.5, 38.2, 34.8, 31.4; HRMS (APCI) calcd. For $\mathrm{C}_{19} \mathrm{H}_{20} \mathrm{O}_{2} \mathrm{Br}(\mathrm{M}+\mathrm{H})$ : 359.0641, Found: 359.0642 .<smiles>O=C1OC(Br)(c2ccc(F)cc2)c2ccccc21</smiles> 
(R)-3-(Bromomethyl)-3-(4-fluorophenyl)isobenzofuran-1(3H)-one (9c): The titled compound was followed by using (R)-1-(bromomethyl)-1-(4-fluorophenyl)-1,3dihydroisobenzofuran (3f) (61.4 mg, $0.20 \mathrm{mmol})$, NBS (2a) (142.4 mg, $0.8 \mathrm{mmol}$ ). Purification by column chromatography (Petroleum ether/EtOAc $=10 / 1)$ yielded 9c $\left(63.7 \mathrm{mg}, 99 \%\right.$ yield, $78 \%$ ee) as pale yellow oil. $[\alpha]_{\mathrm{D}}^{30}=-17.2\left(c 0.43, \mathrm{CHCl}_{3}\right) ;{ }^{1} \mathrm{H}$ NMR (300 MHz, $\left.\mathrm{CDCl}_{3}, \mathrm{ppm}\right) \delta 7.95(\mathrm{~d}, J=7.5 \mathrm{~Hz}, 1 \mathrm{H}), 7.80-7.72(\mathrm{~m}, 1 \mathrm{H}), 7.69-7.60$ $(\mathrm{m}, 2 \mathrm{H}), 7.60-7.51(\mathrm{~m}, 2 \mathrm{H}), 7.14-7.04(\mathrm{~m}, 2 \mathrm{H}), 4.10(\mathrm{~d}, J=11.4 \mathrm{~Hz}, 1 \mathrm{H}), 4.04(\mathrm{~d}, J=$ $11.4 \mathrm{~Hz}, 1 \mathrm{H}) ;{ }^{13} \mathrm{C} \mathrm{NMR}\left(100 \mathrm{MHz}, \mathrm{CDCl}_{3}, \mathrm{ppm}\right) \delta 168.8,163.2(\mathrm{~d}, J=248.0 \mathrm{~Hz})$, $149.7,134.7,133.5(\mathrm{~d}, J=3.0 \mathrm{~Hz}), 130.3,127.8(\mathrm{~d}, J=9.0 \mathrm{~Hz}), 126.7,126.4,122.8$, $116.2(\mathrm{~d}, J=21.0 \mathrm{~Hz}), 87.0,38.0 ;{ }^{19} \mathrm{~F}$ NMR $\left(376 \mathrm{MHz}, \mathrm{CDCl}_{3}, \mathrm{ppm}\right) \delta-120.0$.

Okada, M.; Kaneko, K.; Yamanaka, M.; Shirakawa, S. Org. Biomol. Chem. 2019, 17, $3747-3751$.

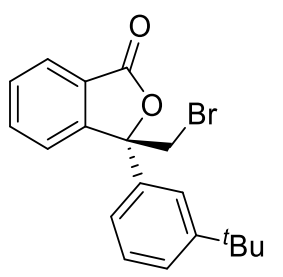

(R)-3-(Bromomethyl)-3-(3-(tert-butyl)phenyl)isobenzofuran-1(3H)-one (9d): The titled compound was followed by using (R)-1-(bromomethyl)-1-(3-(tert-butyl)phenyl)1,3-dihydroisobenzofuran (3k) (69.1 mg, $0.20 \mathrm{mmol})$, NBS (2a) (142.4 mg, $0.8 \mathrm{mmol})$. Purification by column chromatography (Petroleum ether/EtOAc $=40 / 1)$ yielded 9d (68.7 mg, 96\% yield, $74 \%$ ee) as pale yellow oil. $[\alpha]_{\mathrm{D}}^{31}=-4.1\left(c 0.17, \mathrm{CHCl}_{3}\right) ; \operatorname{IR}($ film): 2963, 2921, 1775, 1084, $768 \mathrm{~cm}^{-1} ;{ }^{1} \mathrm{H}$ NMR (400 MHz, $\left.\mathrm{CDCl}_{3}, \mathrm{ppm}\right) \delta 7.94(\mathrm{~d}, J=7.6$ 
$\mathrm{Hz}, 1 \mathrm{H}), 7.78-7.71(\mathrm{~m}, 1 \mathrm{H}), 7.67(\mathrm{~d}, J=7.6 \mathrm{~Hz}, 1 \mathrm{H}), 7.63-7.56(\mathrm{~m}, 2 \mathrm{H}), 7.43-7.29(\mathrm{~m}$, 3H), $4.16(\mathrm{~d}, J=11.6 \mathrm{~Hz}, 1 \mathrm{H}), 4.09(\mathrm{~d}, J=11.2 \mathrm{~Hz}, 1 \mathrm{H}), 1.31(\mathrm{~s}, 9 \mathrm{H}) ;{ }^{13} \mathrm{C}$ NMR $(100$ $\left.\mathrm{MHz}, \mathrm{CDCl}_{3}, \mathrm{ppm}\right) \delta 169.2,152.4,150.1,137.3,134.5,130.1,128.8,127.0,126.5$, 126.2, 122.8, 122.7, 122.6, 87.8, 38.5, 35.2, 31.5; HRMS (APCI) calcd. For $\mathrm{C}_{19} \mathrm{H}_{20} \mathrm{O}_{2} \mathrm{Br}$ $(\mathrm{M}+\mathrm{H}): 359.0641$, Found: 359.0639 .

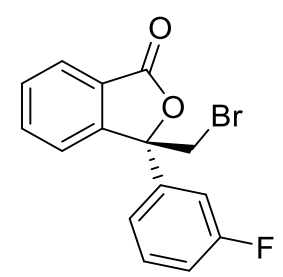

(R)-3-(Bromomethyl)-3-(3-fluorophenyl)isobenzofuran-1(3H)-one (9e): The titled compound was followed by using (R)-1-(bromomethyl)-1-(3-fluorophenyl)-1,3dihydroisobenzofuran (3m) (61.4 mg, $0.20 \mathrm{mmol})$, NBS (2a) (142.4 mg, $0.8 \mathrm{mmol})$. Purification by column chromatography $($ Petroleum ether/EtOAc $=10 / 1)$ yielded 9e $(62.7 \mathrm{mg}, 98 \%$ yield, $83 \%$ ee $)$ as pale yellow oil. $[\alpha]_{\mathrm{D}}^{30}=+5.0\left(c 0.90, \mathrm{CHCl}_{3}\right) ;{ }^{1} \mathrm{H} \mathrm{NMR}$ (400 MHz, $\left.\mathrm{CDCl}_{3}, \mathrm{ppm}\right) \delta 7.95(\mathrm{~d}, J=7.6 \mathrm{~Hz}, 1 \mathrm{H}), 7.80-7.72(\mathrm{~m}, 1 \mathrm{H}), 7.70-7.57(\mathrm{~m}$, 2H), 7.45-7.33 (m, 2H), 7.32-7.24 (m, 1H), 7.12-7.02 (m, 1H), $4.11(\mathrm{~d}, J=11.2 \mathrm{~Hz}$ 1H), $4.05(\mathrm{~d}, J=11.2 \mathrm{~Hz}, 1 \mathrm{H}) ;{ }^{13} \mathrm{C} \mathrm{NMR}\left(100 \mathrm{MHz}, \mathrm{CDCl}_{3}, \mathrm{ppm}\right) \delta 168.7,163.1(\mathrm{~d}, J$ $=246.0 \mathrm{~Hz}), 149.5,140.2(\mathrm{~d}, J=6.0 \mathrm{~Hz}), 134.8,130.9(\mathrm{~d}, J=8.0 \mathrm{~Hz}), 130.4,126.6$, 126.4, 122.7, $121.4(\mathrm{~d}, J=3.0 \mathrm{~Hz}), 116.4(\mathrm{~d}, J=21.0 \mathrm{~Hz}), 113.3(\mathrm{~d}, J=24.0 \mathrm{~Hz}), 86.8$, $37.7 ;{ }^{19} \mathrm{~F}$ NMR $\left(376 \mathrm{MHz}, \mathrm{CDCl}_{3}, \mathrm{ppm}\right) \delta-110.7$.

Okada, M.; Kaneko, K.; Yamanaka, M.; Shirakawa, S. Org. Biomol. Chem. 2019, 17, $3747-3751$. 
<smiles>O=C1OC(CBr)(c2cccs2)c2ccccc21</smiles>

(S)-3-(Bromomethyl)-3-(thiophen-2-yl)isobenzofuran-1(3H)-one (9f): The titled compound was followed by using (S)-1-(bromomethyl)-1-(thiophen-2-yl)-1,3dihydroisobenzofuran (3o) (59.0 mg, $0.20 \mathrm{mmol})$, NBS (2a) (142.4 mg, $0.8 \mathrm{mmol})$. Purification by column chromatography (Petroleum ether/EtOAc $=20 / 1$ ) yielded 9f (55.6 mg, 90\% yield, $52 \%$ ee) as pale red oil. $[\alpha]_{\mathrm{D}}^{32}=-31.9\left(c 0.32, \mathrm{CHCl}_{3}\right)$; IR (film): 1771, 1653, 1216, $756 \mathrm{~cm}^{-1} ;{ }^{1} \mathrm{H}$ NMR $\left(300 \mathrm{MHz}, \mathrm{CDCl}_{3}, \mathrm{ppm}\right) \delta 7.98(\mathrm{~d}, J=7.8 \mathrm{~Hz}$, 1H), 7.85-7.75 (m, 1H), 7.72-7.63 (m, 2H), $6.99(\mathrm{~d}, J=3.9 \mathrm{~Hz}, 1 \mathrm{H}), 6.93(\mathrm{~d}, J=3.6$ $\mathrm{Hz}, 1 \mathrm{H}), 4.12(\mathrm{~d}, J=11.4 \mathrm{~Hz}, 1 \mathrm{H}), 4.06(\mathrm{~d}, J=11.1 \mathrm{~Hz}, 1 \mathrm{H}) ;{ }^{13} \mathrm{C}$ NMR $(100 \mathrm{MHz}$, $\left.\mathrm{CDCl}_{3}, \mathrm{ppm}\right) \delta 168.2,148.8,141.8,134.8,130.8,130.3,126.9,126.7,126.4,122.7$, 114.4, 84.9, 37.4; HRMS (APCI) calcd. For $\mathrm{C}_{13} \mathrm{H}_{8} \mathrm{O}_{2} \mathrm{BrS}(\mathrm{M}-\mathrm{H})$ : 306.9423, Found: 306.9421 . 
By slow evaporation in the mixture of $\mathrm{CH}_{2} \mathrm{Cl}_{2}$ and $n$-hexane, single crystal of $\mathbf{3 a}$ was obtained.

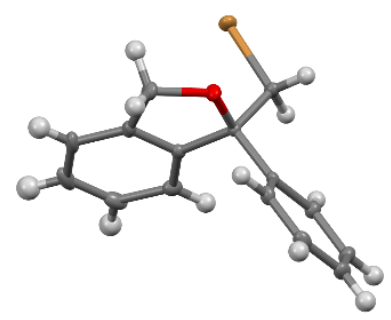

ORTEP drawing of $\mathbf{3 a}$ (the thermal ellipsoids are displayed at $30 \%$ probability).

Table 1 Crystal data and structure refinement for $3 \mathrm{a}$.

Identification code

MX8635

Empirical formula

$\mathrm{C}_{15} \mathrm{H}_{13} \mathrm{BrO}$

Formula weight

289.16

Temperature/K

170.01(10)

Crystal system

orthorhombic

Space group

$\mathrm{P} 2{ }_{1} 2{ }_{1} 2_{1}$

$\mathrm{a} / \AA \AA$

6.9956(2)

$\mathrm{b} / \AA$

8.1261(2)

$\mathrm{c} / \AA$

21.4487(5)

$\alpha /{ }^{\circ}$

90

$\beta /{ }^{\circ}$

90

$\gamma /{ }^{\circ}$

90

Volume $/ \AA^{3}$

1219.29(5)

Z

4

$\rho_{\text {calc }} \mathrm{g} / \mathrm{cm}^{3}$

1.575

$\mu / \mathrm{mm}^{-1}$

3.351

$\mathrm{F}(000)$

584.0

Crystal size $/ \mathrm{mm}^{3}$

$0.31 \times 0.28 \times 0.15$

Radiation

$\operatorname{MoK} \alpha(\lambda=0.71073)$

$2 \Theta$ range for data collection $/{ }^{\circ} 3.798$ to 62.812

Index ranges

$-10 \leq \mathrm{h} \leq 9,-11 \leq \mathrm{k} \leq 10,-26 \leq 1 \leq 29$

Reflections collected

19583

Independent reflections

$3529\left[\mathrm{R}_{\text {int }}=0.0195, \mathrm{R}_{\text {sigma }}=0.0155\right]$

Data/restraints/parameters

$3529 / 0 / 154$

Goodness-of-fit on $\mathrm{F}^{2}$

1.046

Final $R$ indexes $[\mathrm{I}>=2 \sigma(\mathrm{I})] \quad \mathrm{R}_{1}=0.0215, \mathrm{wR}_{2}=0.0492$

Final R indexes [all data] $\quad \mathrm{R}_{1}=0.0242, \mathrm{wR}_{2}=0.0498$

Largest diff. peak/hole / e $\AA^{-3} 0.40 /-0.29$

Flack parameter

$0.084(3)$ 
The chromatography for the determination of the enantiomeric excess<smiles>BrCC1(c2ccccc2)OCc2ccccc21</smiles>

3a

HPLC Conditions: Column: Chiralcel OD-H, Daicel Chemical Industries, Ltd., Eluent: Hexanes/IPA (99/1); Flow rate: $1.0 \mathrm{~mL} / \mathrm{min}$; Detection: UV $210 \mathrm{~nm}$

Racemic

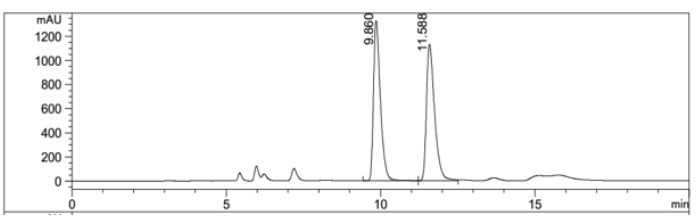

|Peak| RT | Area |Area \% |

| \# | [min] |------ |----|

$|---|---1---\mid$

| $1|9.860| 2.053 \mathrm{e} 4|49.709|$

| 2 | $11.588|2.077 \mathrm{e} 4| 50.291 \mid$
Chiral
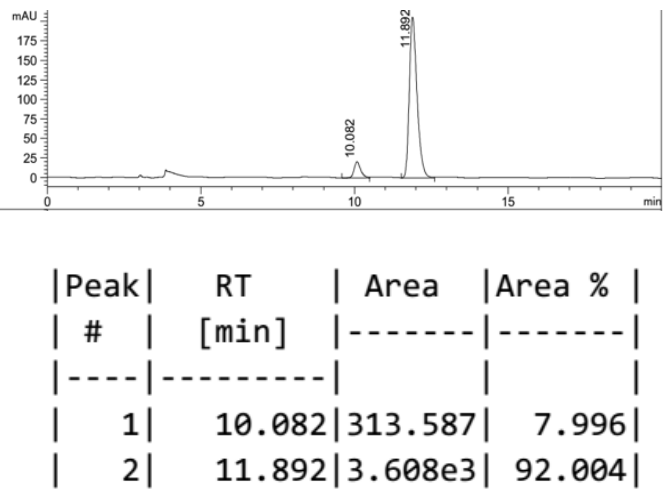<smiles>Cc1ccc(C2(CBr)OCc3ccccc32)cc1</smiles>

3b

HPLC Conditions: Column: Chiralcel OD-H, Daicel Chemical Industries, Ltd., Eluent: Hexanes/IPA (99/1); Flow rate: $1.0 \mathrm{~mL} / \mathrm{min}$; Detection: UV $210 \mathrm{~nm}$

Racemic
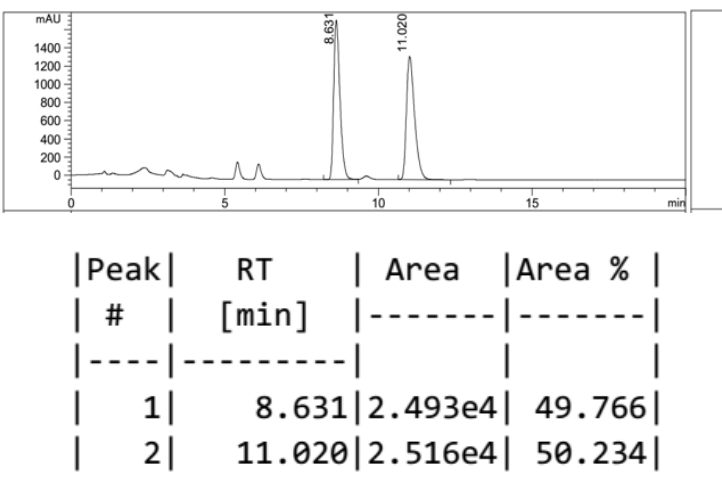

Chiral
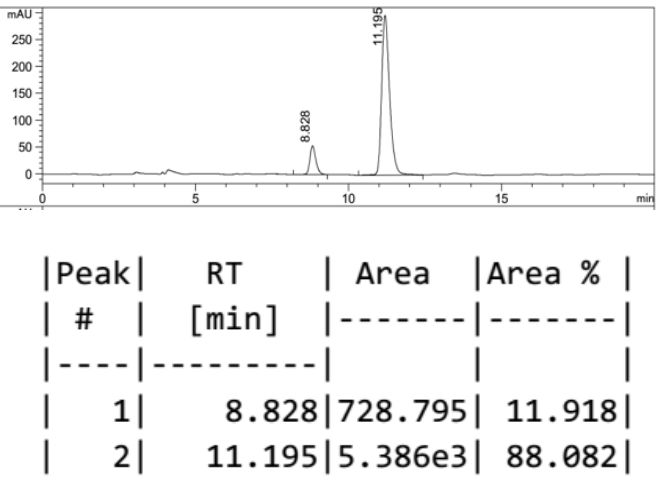


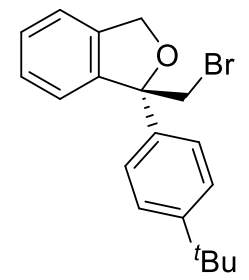

$3 c$

HPLC Conditions: Column: Chiralcel OD-H, Daicel Chemical Industries, Ltd., Eluent: Hexanes/IPA (99/1); Flow rate: $1.0 \mathrm{~mL} / \mathrm{min}$; Detection: UV $210 \mathrm{~nm}$

\section{Racemic}

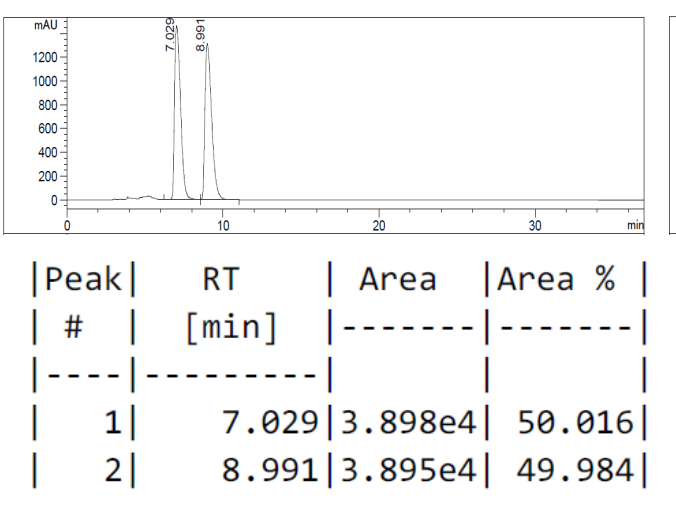

Chiral
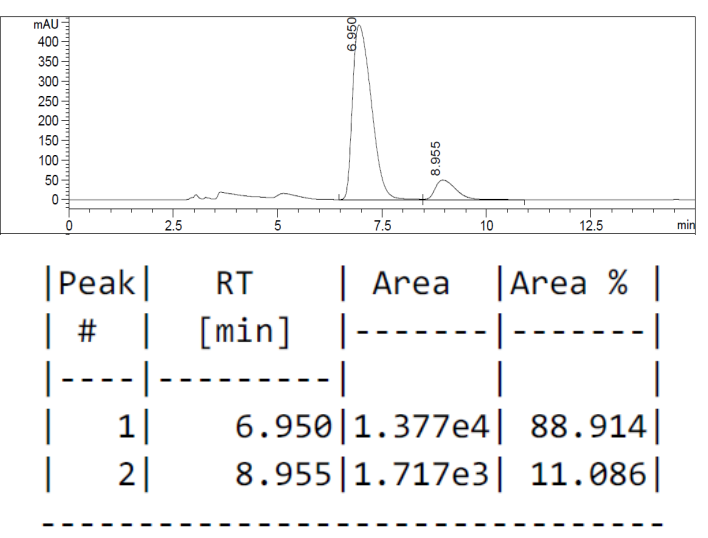<smiles>C=Cc1ccc([C@]2(CBr)OCc3ccccc32)cc1</smiles>

3d

HPLC Conditions: Column: Chiralcel OD-H, Daicel Chemical Industries, Ltd., Eluent: Hexanes/IPA (99/1); Flow rate: $1.0 \mathrm{~mL} / \mathrm{min}$; Detection: UV $254 \mathrm{~nm}$

\section{Racemic}
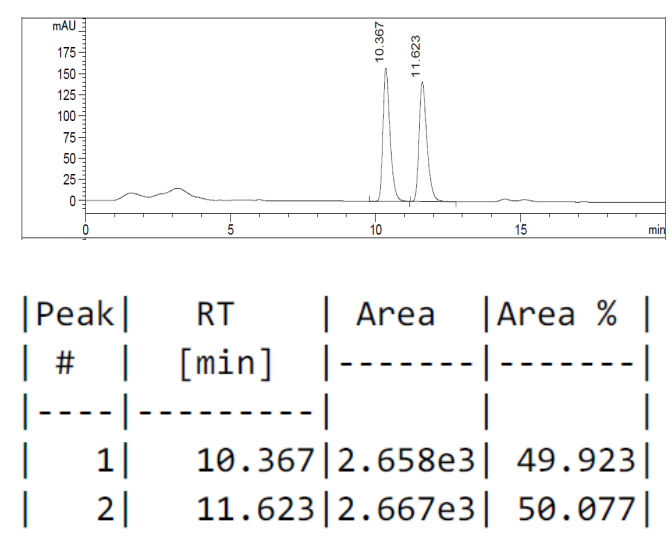

Chiral
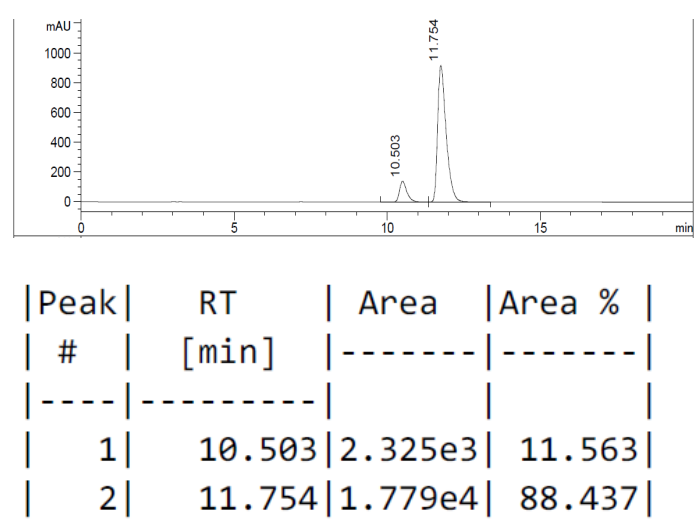
<smiles>BrCC1(c2ccc(-c3ccccc3)cc2)OCc2ccccc21</smiles>

$3 e$

HPLC Conditions: Column: Chiralcel OD-H, Daicel Chemical Industries, Ltd., Eluent: Hexanes/IPA (99/1); Flow rate: $1.0 \mathrm{~mL} / \mathrm{min}$; Detection: UV $254 \mathrm{~nm}$

\section{Racemic}

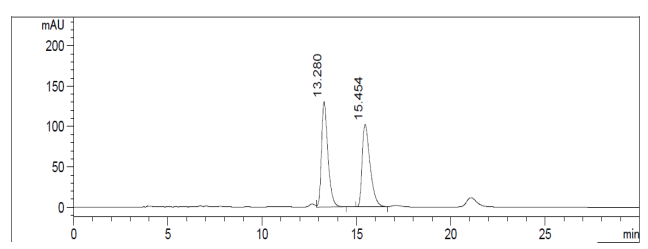

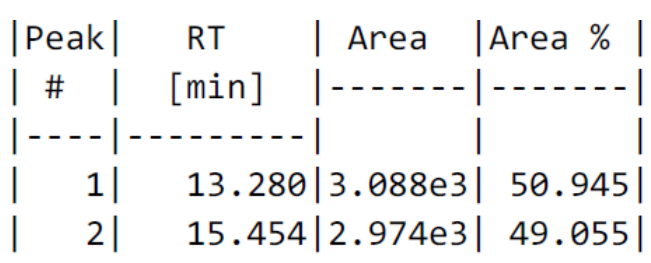

Chiral
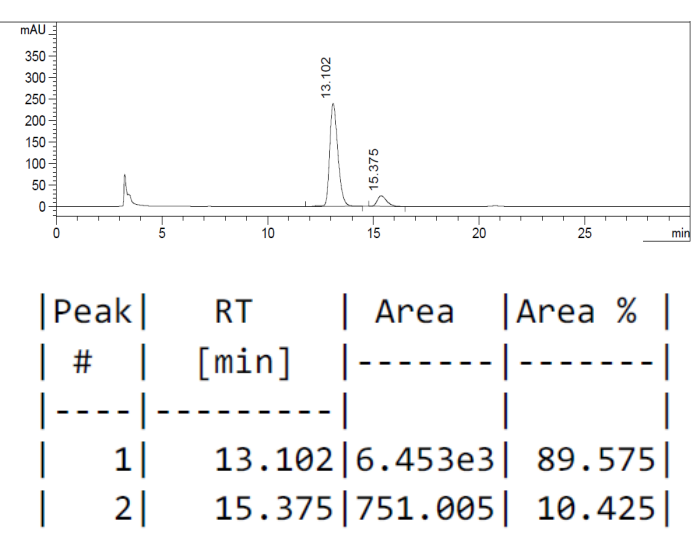<smiles>Fc1ccc(C2(CBr)OCc3ccccc32)cc1</smiles>

$3 f$

HPLC Conditions: Column: Chiralpak AD-H, Daicel Chemical Industries, Ltd., Eluent: Hexanes/IPA (99.5/0.5); Flow rate: $0.6 \mathrm{~mL} / \mathrm{min}$; Detection: UV $210 \mathrm{~nm}$

Racemic

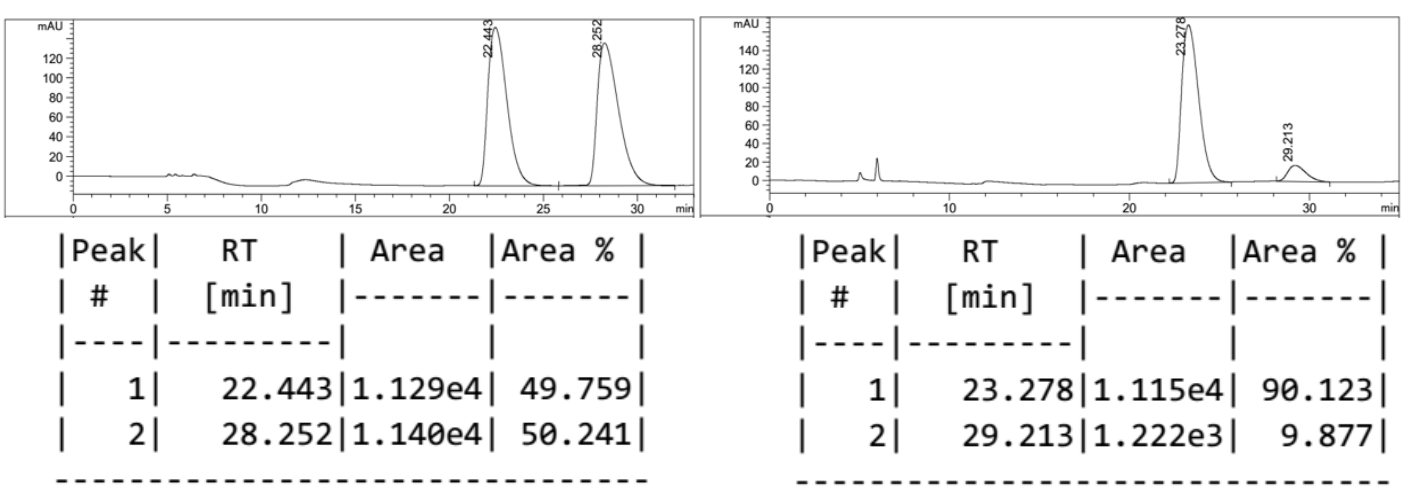




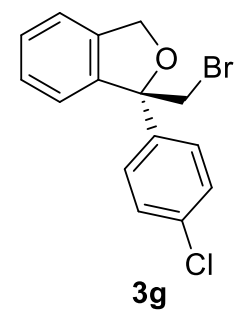

HPLC Conditions: Column: Chiralpak AD-H, Daicel Chemical Industries, Ltd., Eluent: Hexanes/IPA (99/1); Flow rate: $1.0 \mathrm{~mL} / \mathrm{min}$; Detection: UV $210 \mathrm{~nm}$

Racemic

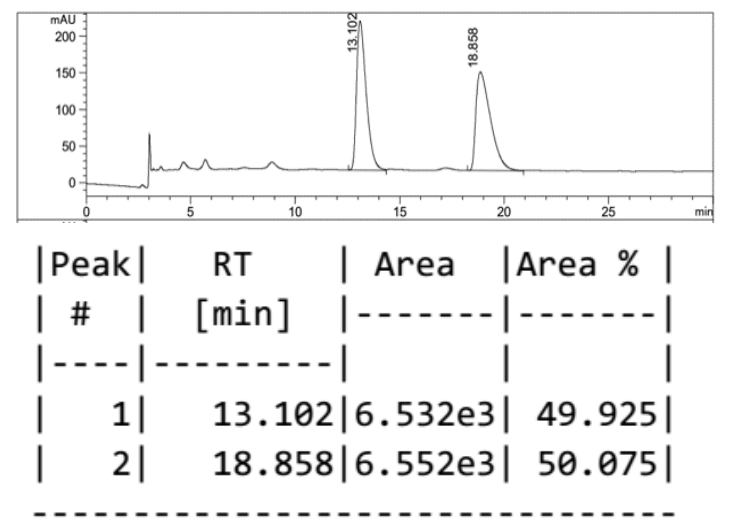

Chiral

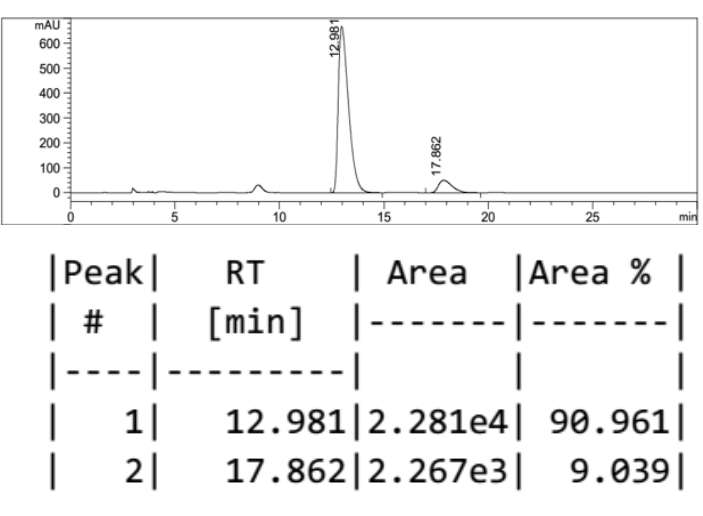

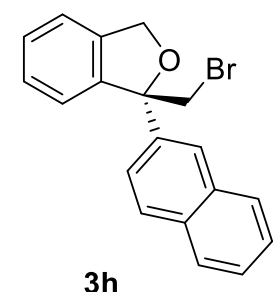

HPLC Conditions: Column: Chiralcel OD-H, Daicel Chemical Industries, Ltd., Eluent: Hexanes/IPA (99/1); Flow rate: $1.0 \mathrm{~mL} / \mathrm{min}$; Detection: UV $210 \mathrm{~nm}$

\section{Racemic}

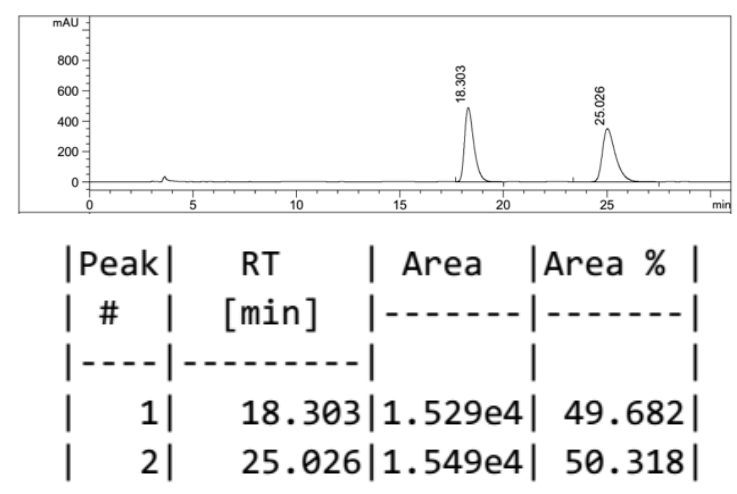

Chiral

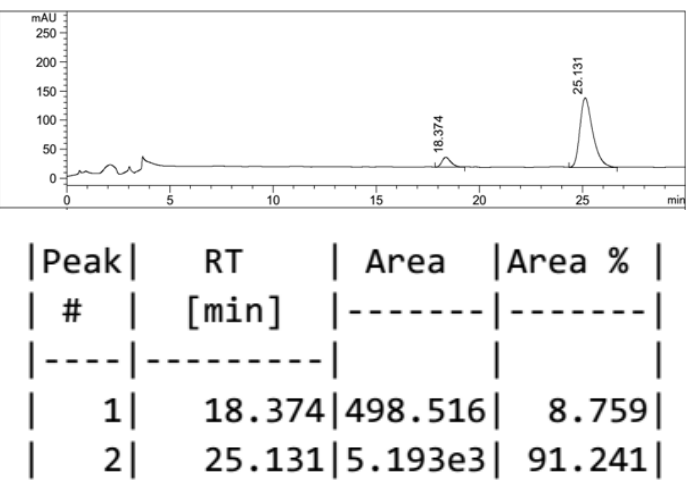




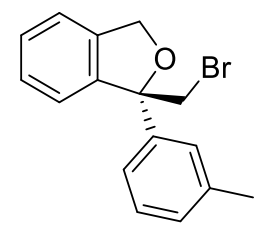

$\mathbf{3 i}$

HPLC Conditions: Column: Chiralcel OD-H, Daicel Chemical Industries, Ltd., Eluent: Hexanes/IPA (99/1); Flow rate: $1.0 \mathrm{~mL} / \mathrm{min}$; Detection: UV $210 \mathrm{~nm}$

\section{Racemic}
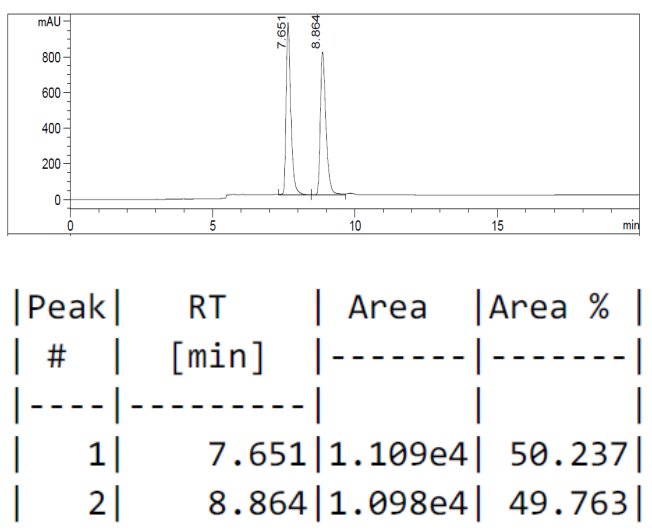

Chiral
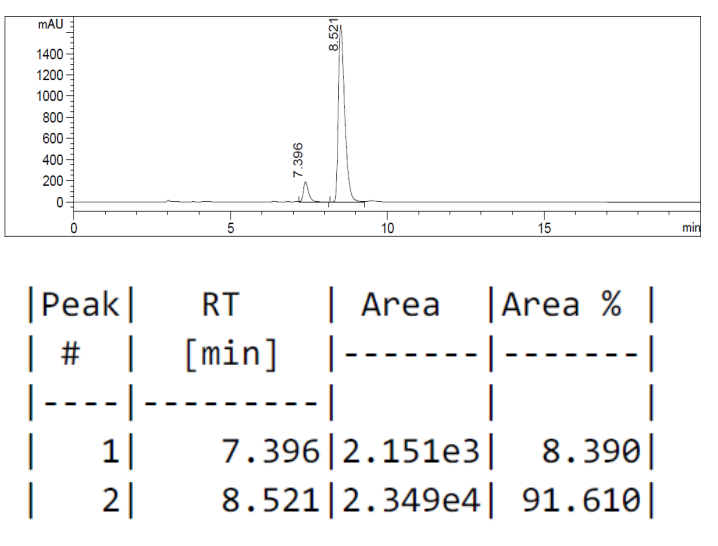

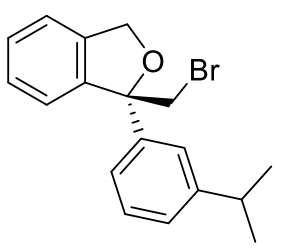

3j

HPLC Conditions: Column: Chiralcel OD-H, Daicel Chemical Industries, Ltd., Eluent: Hexanes/IPA (99/1); Flow rate: $1.0 \mathrm{~mL} / \mathrm{min}$; Detection: UV $210 \mathrm{~nm}$

\section{Racemic}
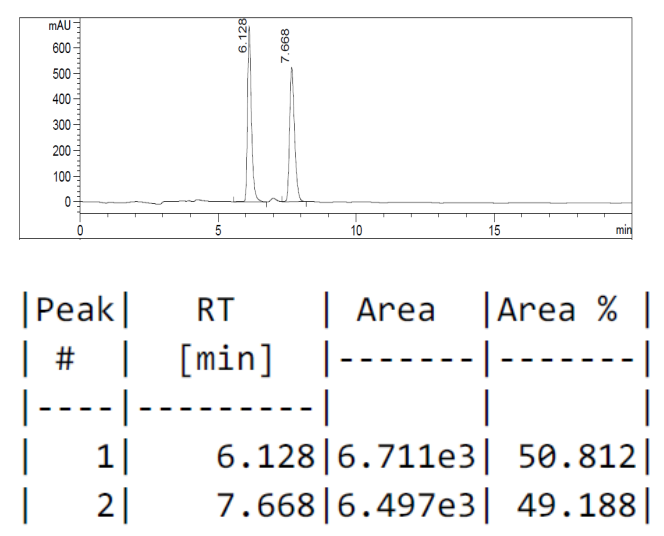

Chiral

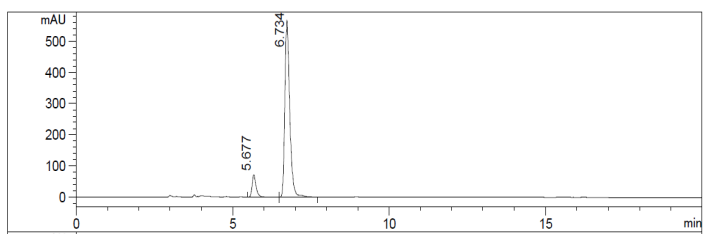

$$
\begin{aligned}
& \text { |Peak | RT | Area |Area \% | } \\
& \text { | \# | [min] |-..-.-....- | } \\
& \text { |---1-n-n| | | } \\
& \text { | } 1|\quad 5.677| 638.654|9.413| \\
& \text { | } 2 \text { | } 6.734|6.146 \mathrm{e} 3| 90.587 \mid
\end{aligned}
$$




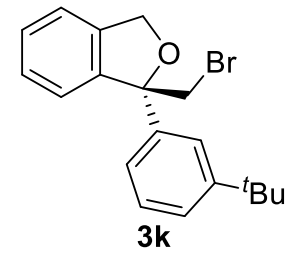

HPLC Conditions: Column: Chiralcel OD-H, Daicel Chemical Industries, Ltd., Eluent: Hexanes/IPA (99/1); Flow rate: $1.0 \mathrm{~mL} / \mathrm{min}$; Detection: UV $210 \mathrm{~nm}$

\section{Racemic}
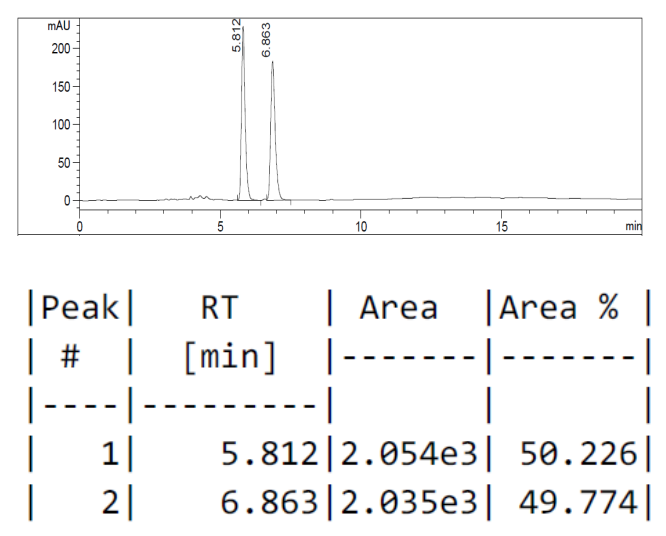

Chiral
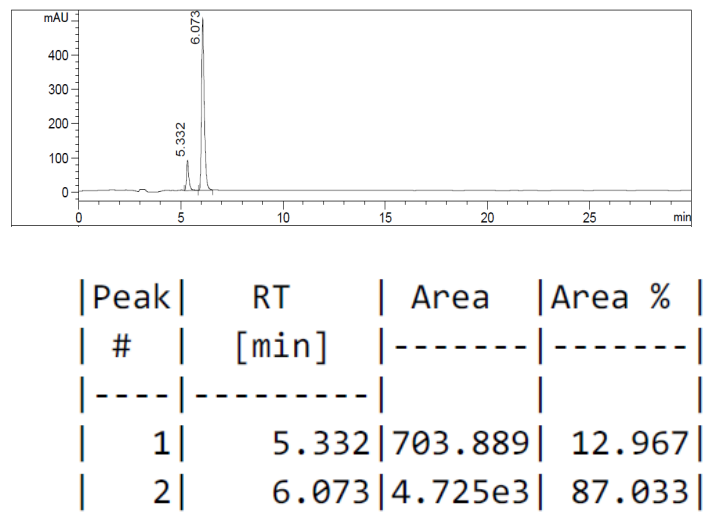<smiles>BrCC1(c2cc[c-]c(-c3ccccc3)c2)OCc2ccccc21</smiles>

HPLC Conditions: Column: Chiralcel OD-H, Daicel Chemical Industries, Ltd., Eluent: Hexanes/IPA (99/1); Flow rate: $1.0 \mathrm{~mL} / \mathrm{min}$; Detection: UV $210 \mathrm{~nm}$

\section{Racemic}

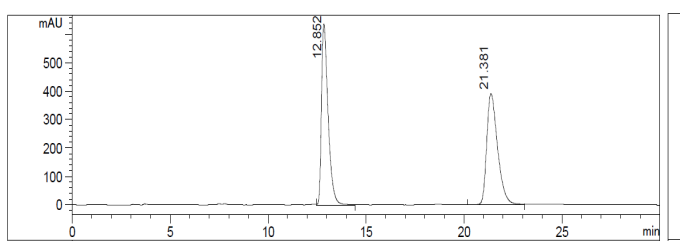

Chiral
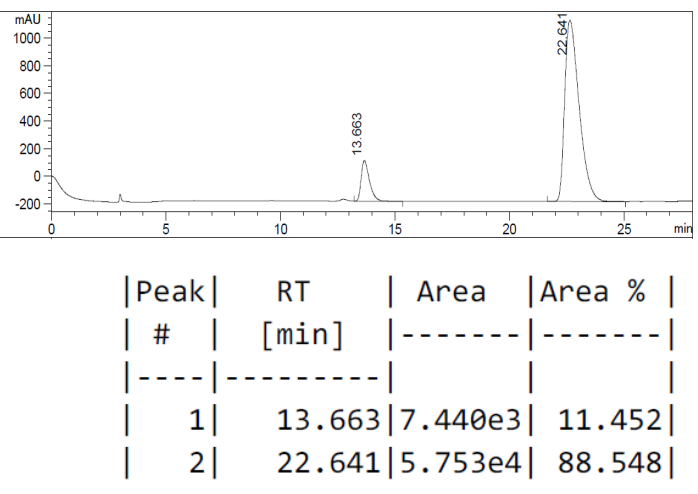


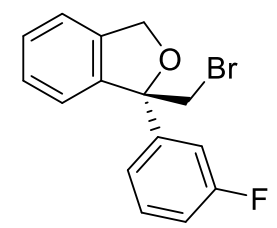

$3 \mathrm{~m}$

HPLC Conditions: Column: Chiralcel OD-H, Daicel Chemical Industries, Ltd., Eluent: Hexanes/IPA (99/1); Flow rate: $1.0 \mathrm{~mL} / \mathrm{min}$; Detection: UV $210 \mathrm{~nm}$

\section{Racemic}
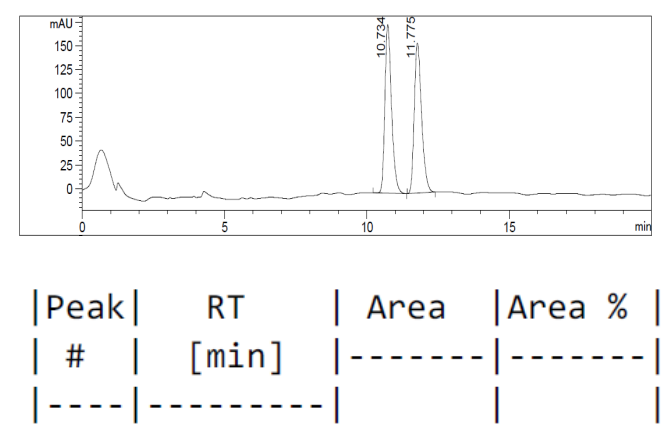

$1|10.734| 2.877 \mathrm{e} 3|50.486|$

2| $11.775|2.822 \mathrm{e} 3| 49.514 \mid$

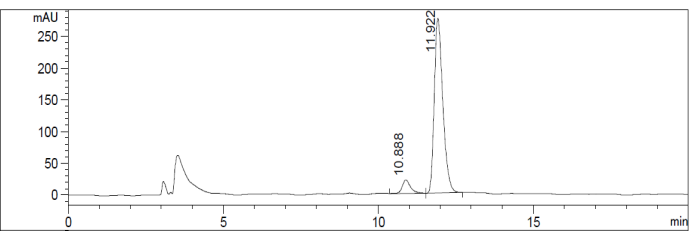

|Peak RT | Area |Area \% |

| \# $\mid$ [min] |-..-.-.-.-.

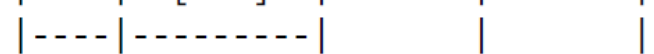

| $1|10.888| 392.705|6.926|$

| 2 | $11.922|5.277 \mathrm{e3}| 93.074 \mid$<smiles>Cc1cc(C)cc([C@]2(CBr)OCc3ccccc32)c1</smiles>

$3 n$

HPLC Conditions: Column: Chiralcel OD-H, Daicel Chemical Industries, Ltd., Eluent: Hexanes/IPA (99/1); Flow rate: $1.0 \mathrm{~mL} / \mathrm{min}$; Detection: UV $210 \mathrm{~nm}$

\section{Racemic}
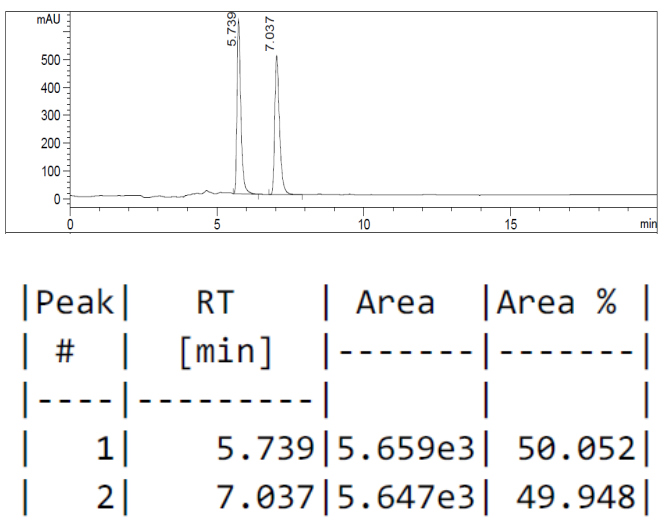

Chiral
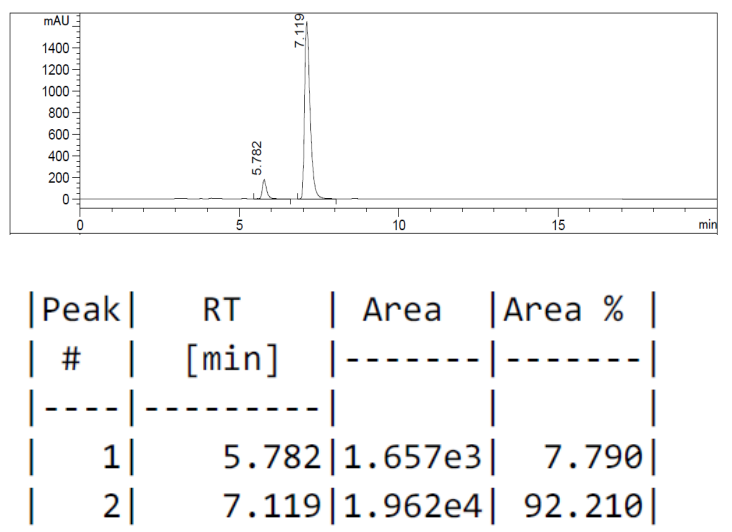


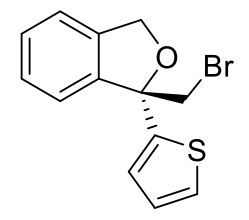

30

HPLC Conditions: Column: Chiralpak IC, Daicel Chemical Industries, Ltd., Eluent: Hexanes/IPA (99/1); Flow rate: $0.5 \mathrm{~mL} / \mathrm{min}$; Detection: UV 230nm

Racemic
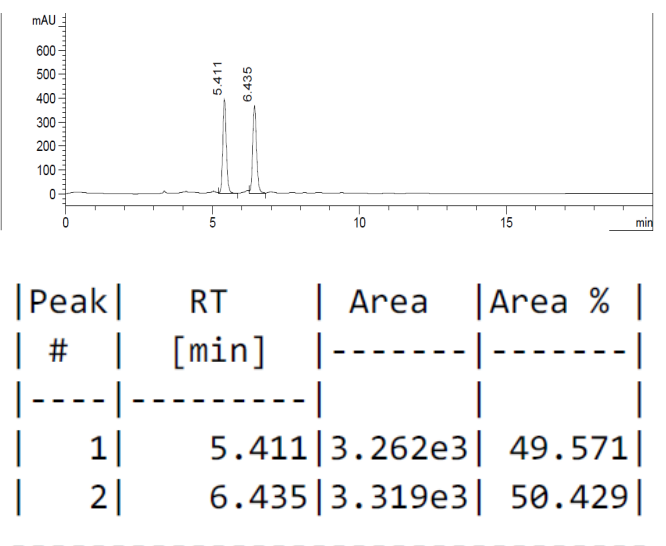

Chiral
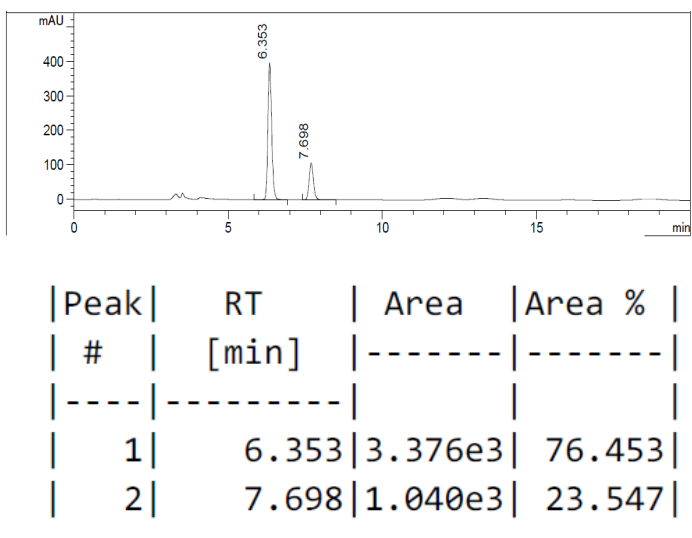<smiles>Fc1ccc2c(c1)COC2(CBr)c1ccccc1</smiles>

$3 p$

HPLC Conditions: Column: Chiralcel OD-H, Daicel Chemical Industries, Ltd., Eluent: Hexanes/IPA (99/1); Flow rate: $1.0 \mathrm{~mL} / \mathrm{min}$; Detection: UV $210 \mathrm{~nm}$

\section{Racemic}
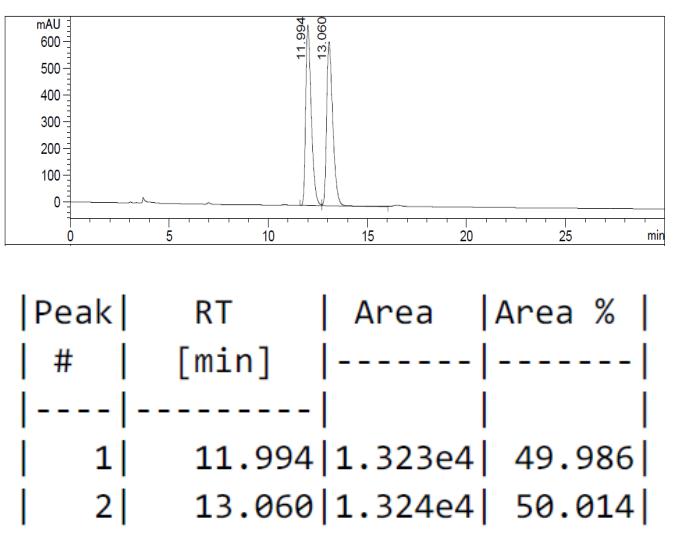

Chiral
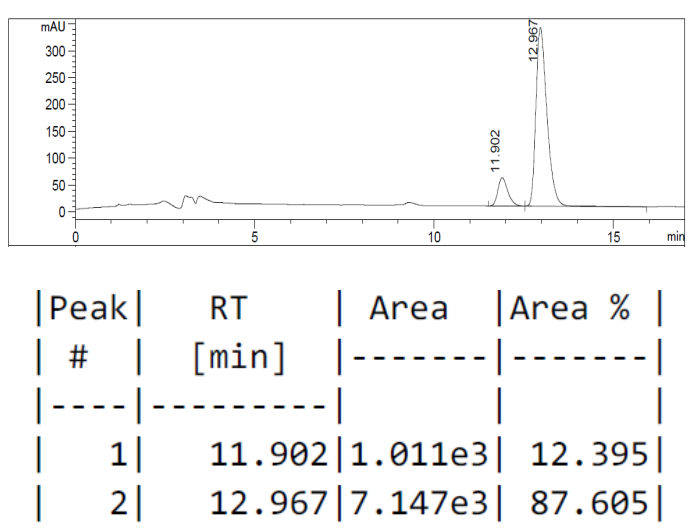
<smiles>N#Cc1ccc2c(c1)COC2(CBr)c1ccc(F)cc1</smiles>

HPLC Conditions: Column: Chiralpak AD-H, Daicel Chemical Industries, Ltd., Eluent: Hexanes/IPA (90/10); Flow rate: $1.0 \mathrm{~mL} / \mathrm{min}$; Detection: UV $210 \mathrm{~nm}$

\section{Racemic}
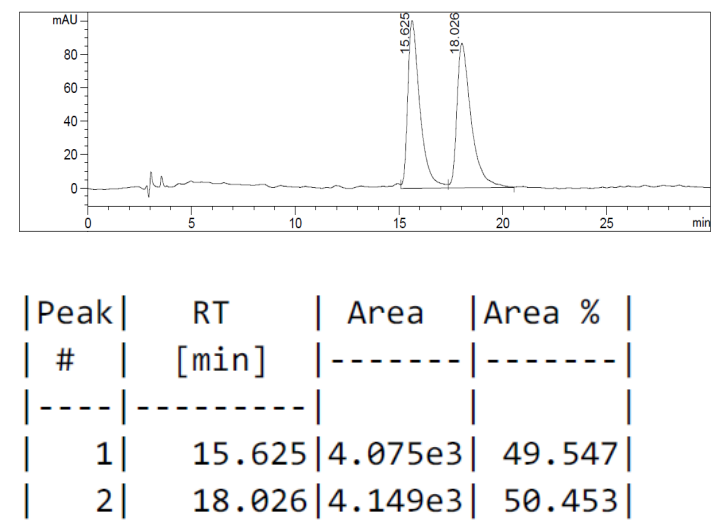

Chiral
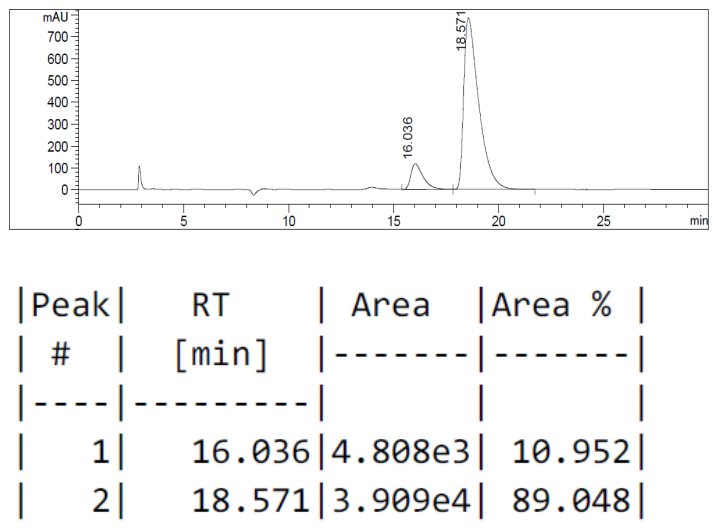<smiles>Fc1ccc2c(c1)C(CBr)(c1ccccc1)OC2</smiles>

$3 r$

HPLC Conditions: Column: Chiralcel OD-H, Daicel Chemical Industries, Ltd., Eluent: Hexanes/IPA (99/1); Flow rate: $1.0 \mathrm{~mL} / \mathrm{min}$; Detection: UV $210 \mathrm{~nm}$

\section{Racemic}
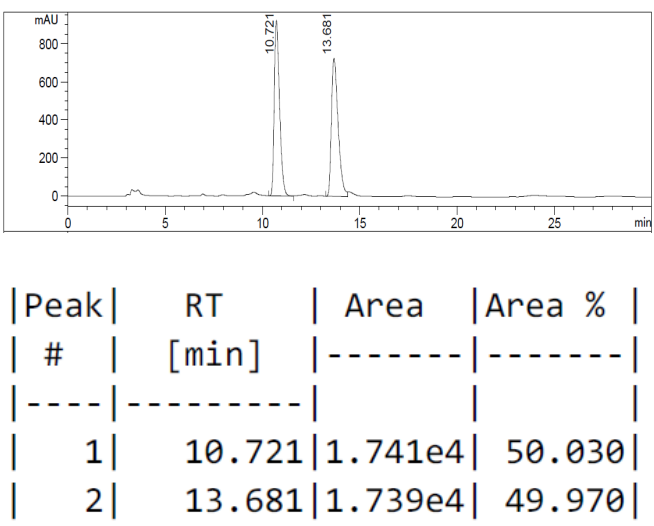

Chiral
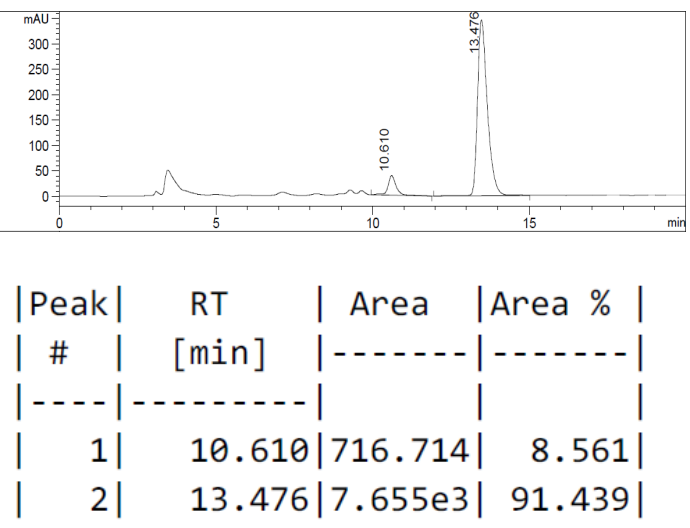


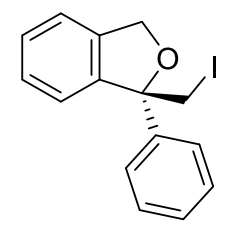

3s

HPLC Conditions: Column: Chiralcel OD-H, Daicel Chemical Industries, Ltd., Eluent: Hexanes/IPA (99/1); Flow rate: $1.0 \mathrm{~mL} / \mathrm{min}$; Detection: UV $210 \mathrm{~nm}$

Racemic
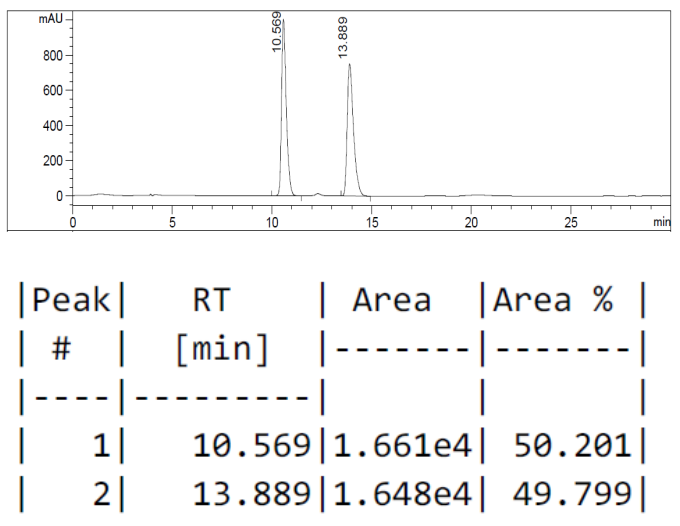

Chiral
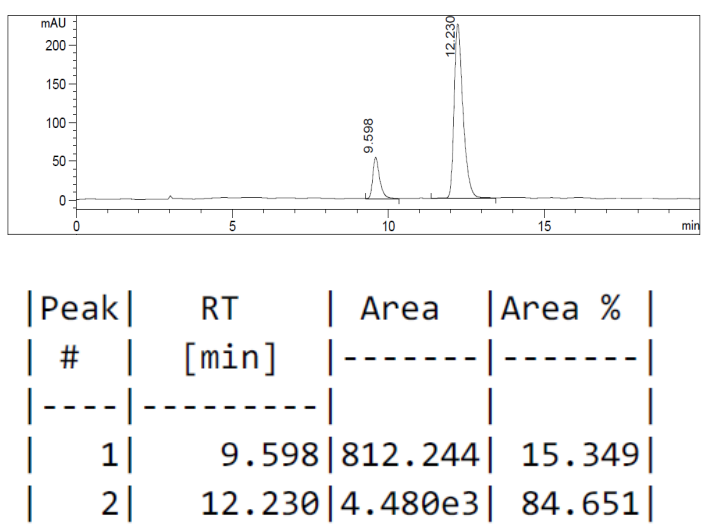<smiles>Cc1cc(C)cc(C2(CI)OCc3ccccc32)c1</smiles>

3t

HPLC Conditions: Column: Chiralcel OD-H, Daicel Chemical Industries, Ltd., Eluent: Hexanes/IPA (99/1); Flow rate: $1.0 \mathrm{~mL} / \mathrm{min}$; Detection: UV $210 \mathrm{~nm}$

Racemic
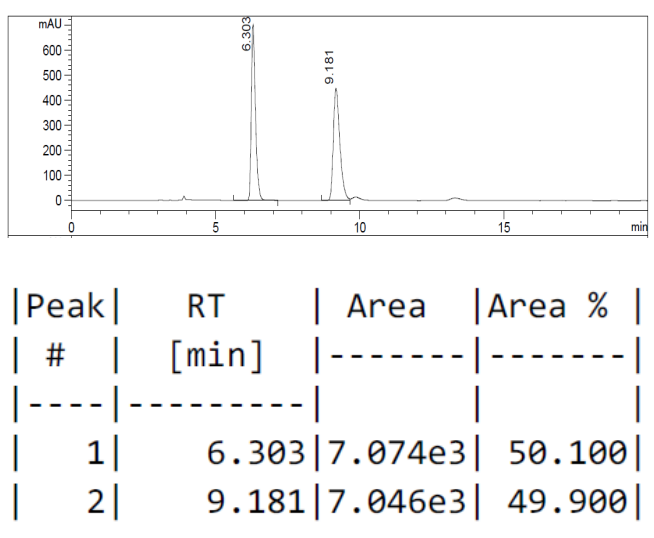

Chiral
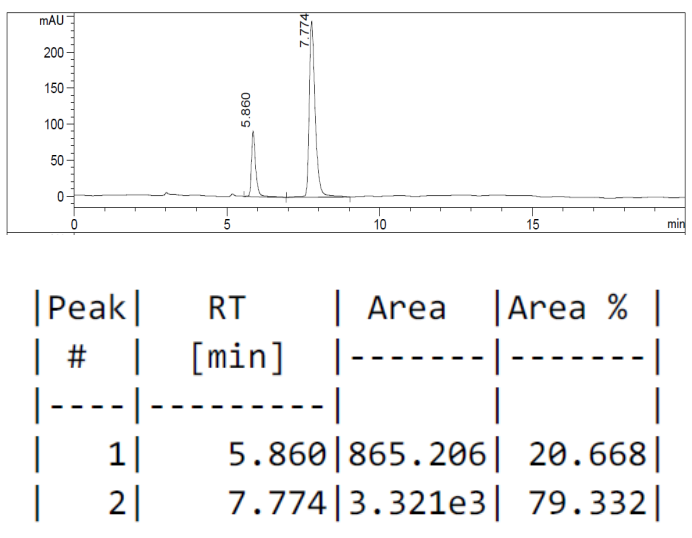


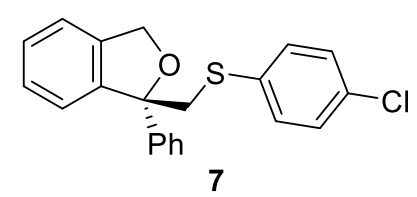

HPLC Conditions: Column: Chiralcel OD-H, Daicel Chemical Industries, Ltd., Eluent: Hexanes/IPA (99.5/0.5); Flow rate: $1.0 \mathrm{~mL} / \mathrm{min}$; Detection: UV $254 \mathrm{~nm}$

\section{Racemic}
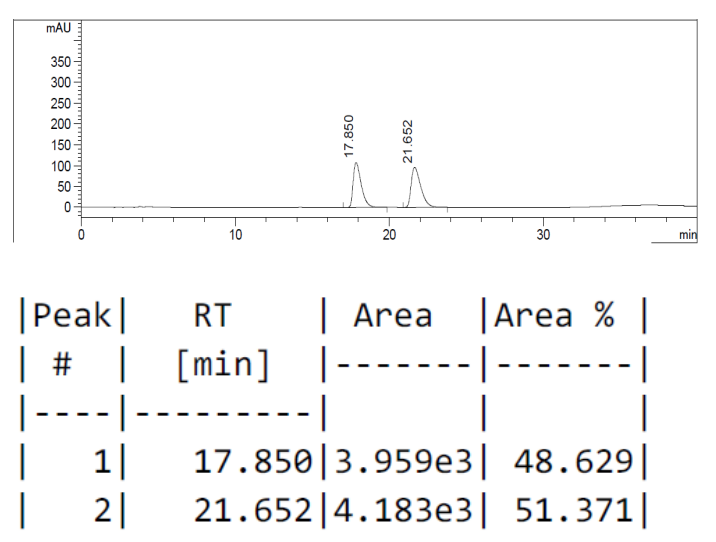

Chiral
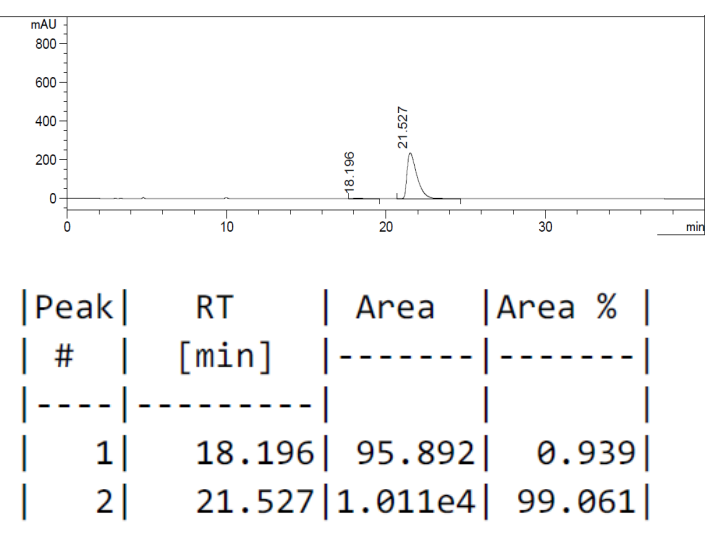

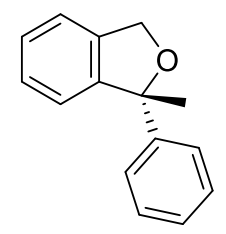

8

HPLC Conditions: Column: Chiralpak AD-H, Daicel Chemical Industries, Ltd., Eluent: Hexanes/IPA (99/1); Flow rate: $1.0 \mathrm{~mL} / \mathrm{min}$; Detection: UV $210 \mathrm{~nm}$

\section{Racemic}
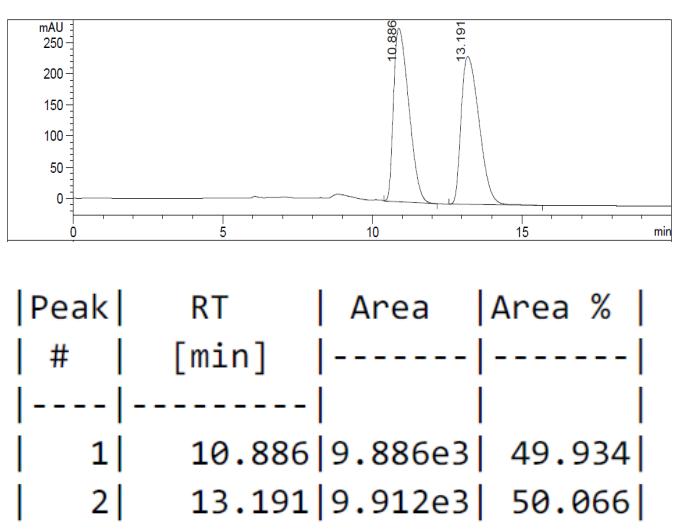

Chiral
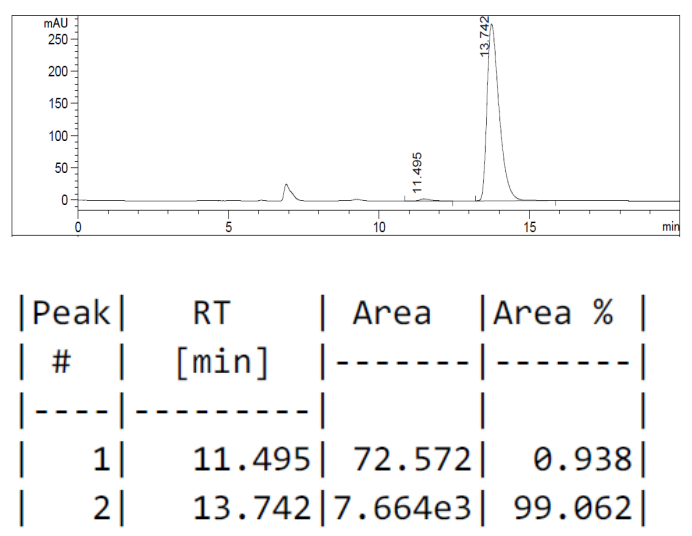


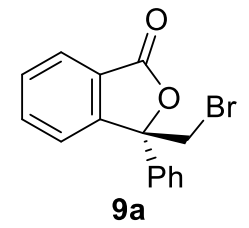

HPLC Conditions: Column: Chiralpak IC, Daicel Chemical Industries, Ltd., Eluent: Hexanes/IPA (67/33); Flow rate: $0.5 \mathrm{~mL} / \mathrm{min}$; Detection: UV $230 \mathrm{~nm}$

\section{Racemic}
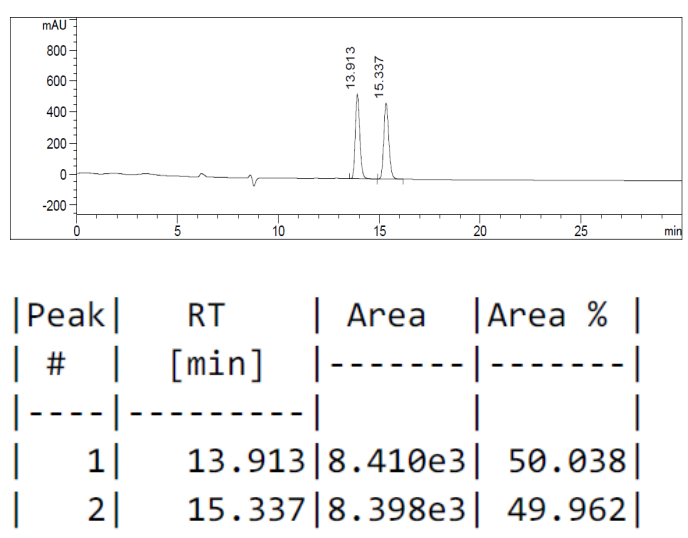

Chiral
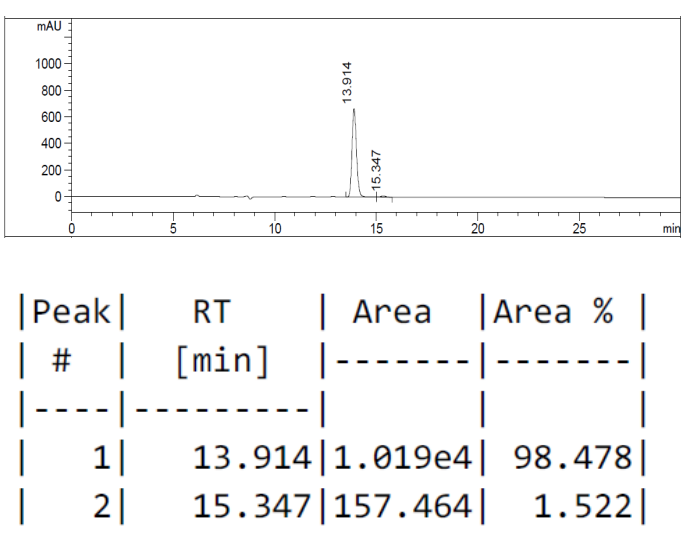<smiles>CC(C)(C)c1ccc([C@]2(CBr)OC(=O)c3ccccc32)cc1</smiles>

$9 b$

HPLC Conditions: Column: Chiralpak IC, Daicel Chemical Industries, Ltd., Eluent: Hexanes/IPA (96/4); Flow rate: $0.6 \mathrm{~mL} / \mathrm{min}$; Detection: UV $230 \mathrm{~nm}$

\section{Racemic}
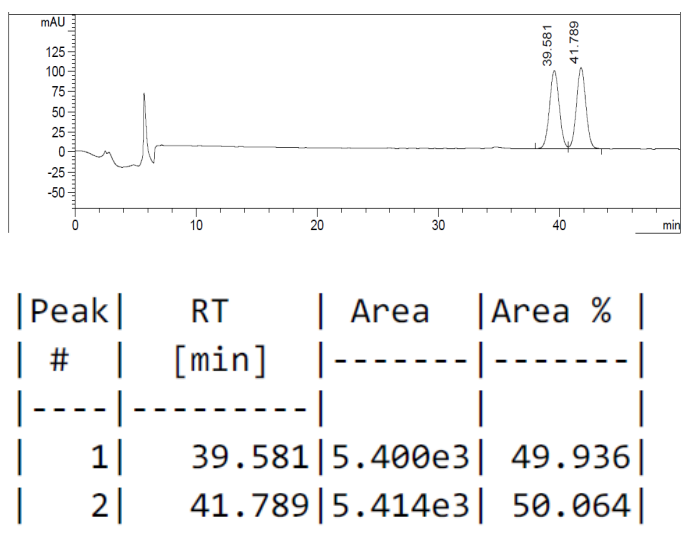

Chiral
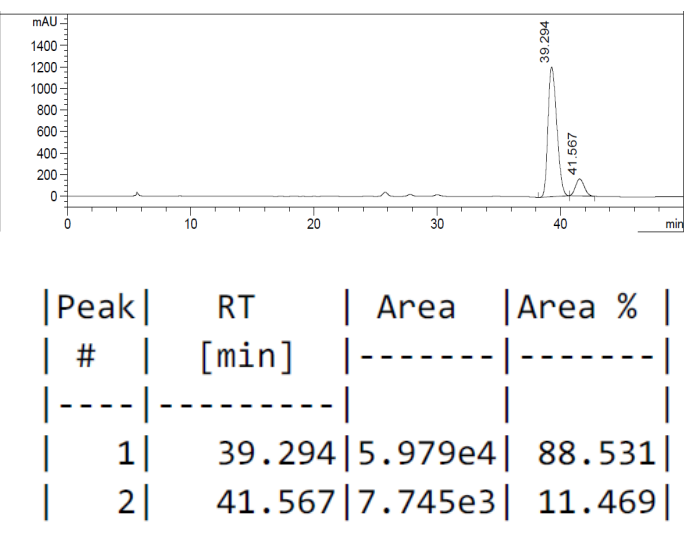


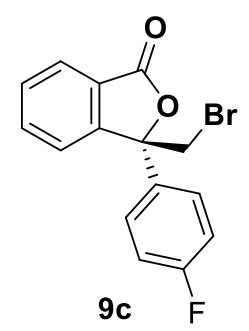

HPLC Conditions: Column: Chiralpak IC, Daicel Chemical Industries, Ltd., Eluent: Hexanes/IPA (70/30); Flow rate: $0.5 \mathrm{~mL} / \mathrm{min}$; Detection: UV $210 \mathrm{~nm}$

\section{Racemic}
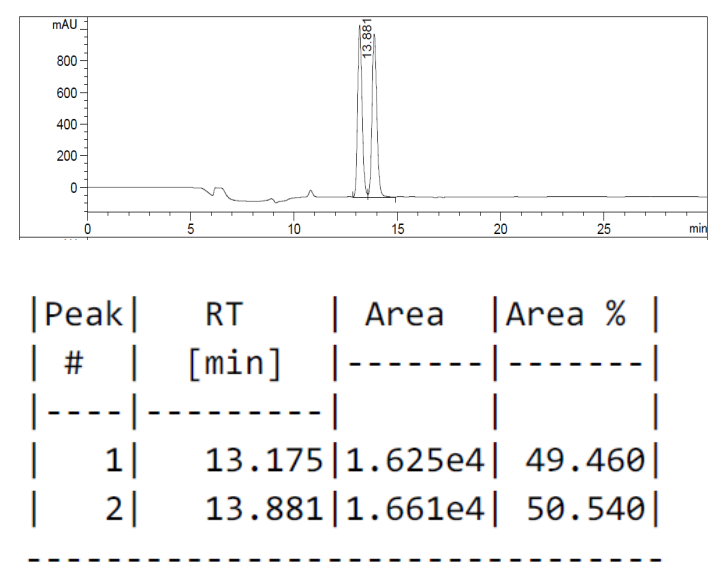

\section{Chiral}
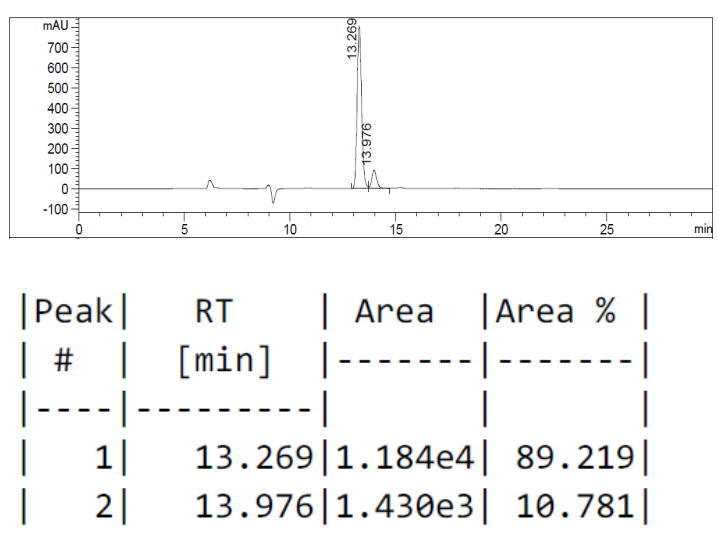<smiles>CC(C)(C)c1cccc([C@]2(CBr)OC(=O)c3ccccc32)c1</smiles>

HPLC Conditions: Column: Chiralpak IC, Daicel Chemical Industries, Ltd., Eluent: Hexanes/IPA (96/4); Flow rate: $0.6 \mathrm{~mL} / \mathrm{min}$; Detection: UV $230 \mathrm{~nm}$

\section{Racemic}
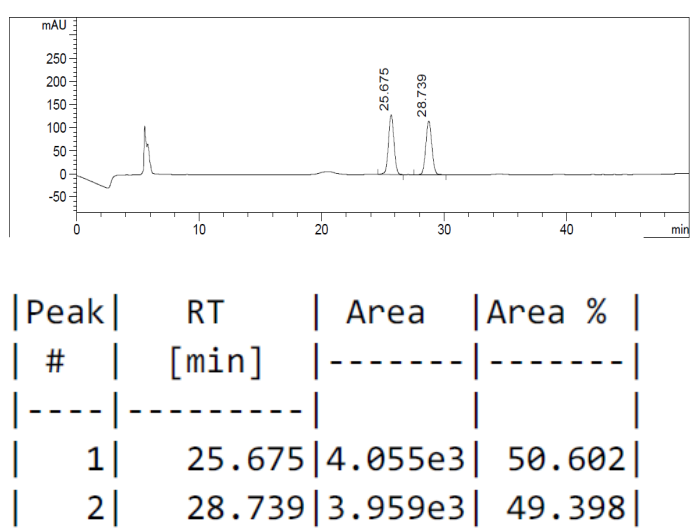

Chiral
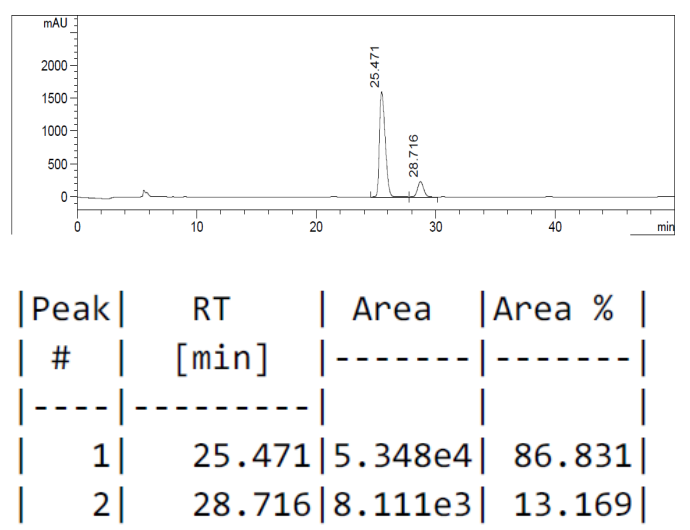
<smiles>O=C1OC(CBr)(c2cccc(F)c2)c2ccccc21</smiles>

$9 e$

HPLC Conditions: Column: Chiralpak IC, Daicel Chemical Industries, Ltd., Eluent: Hexanes/IPA (80/20); Flow rate: $0.5 \mathrm{~mL} / \mathrm{min}$; Detection: UV $230 \mathrm{~nm}$

\section{Racemic}
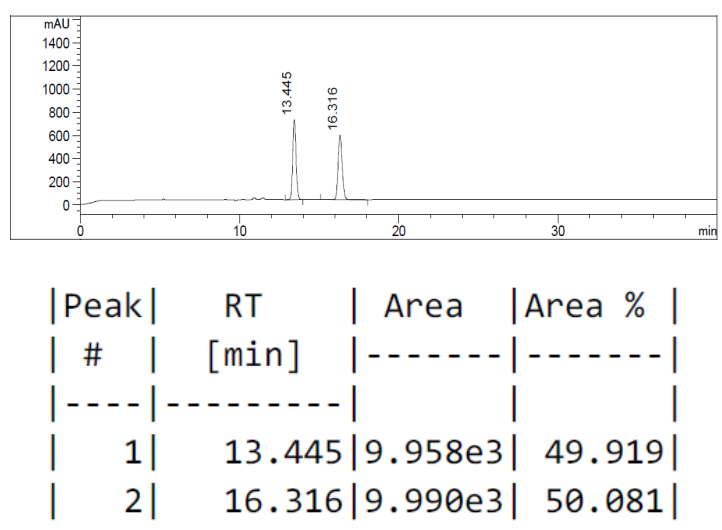<smiles>O=C1OC(CBr)(c2cccs2)c2ccccc21</smiles>

9f
Chiral
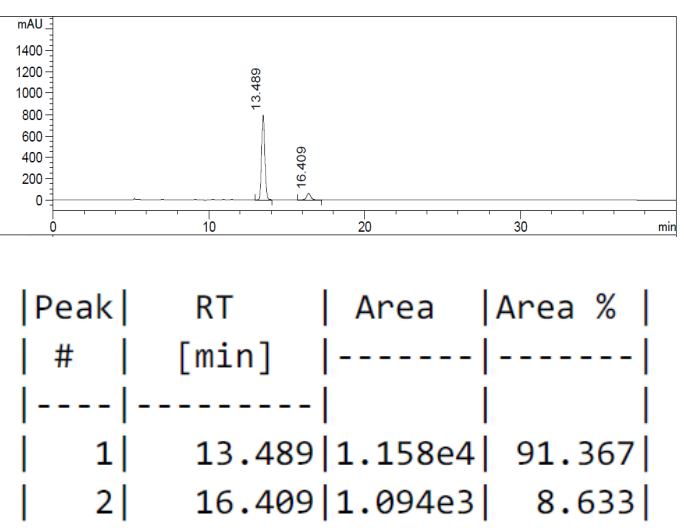

HPLC Conditions: Column: Chiralpak IC, Daicel Chemical Industries, Ltd., Eluent: Hexanes/IPA (96/4); Flow rate: $0.6 \mathrm{~mL} / \mathrm{min}$; Detection: UV $254 \mathrm{~nm}$

\section{Racemic}
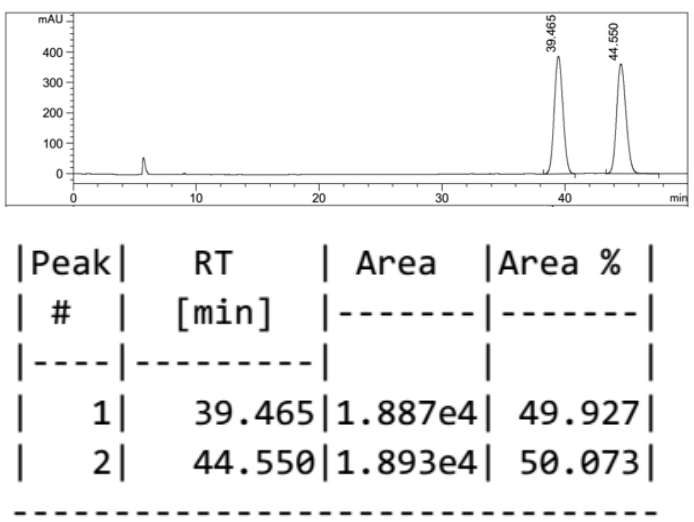

Chiral
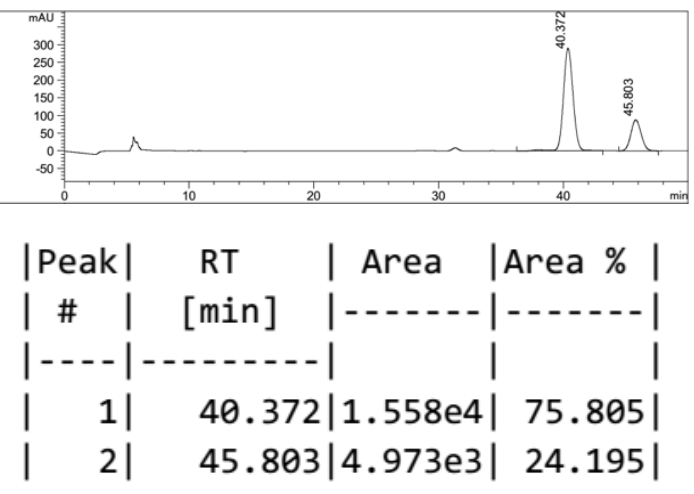


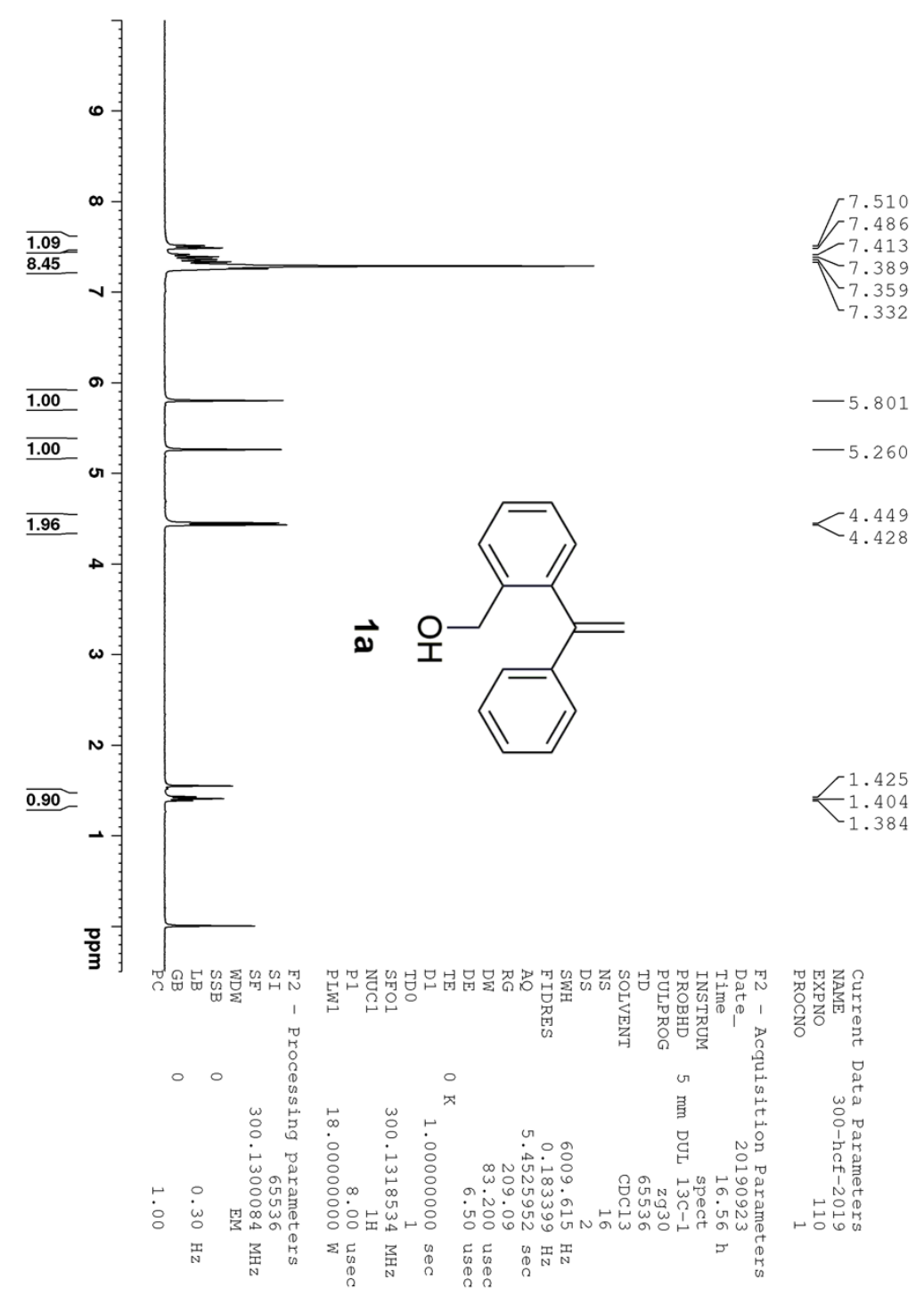




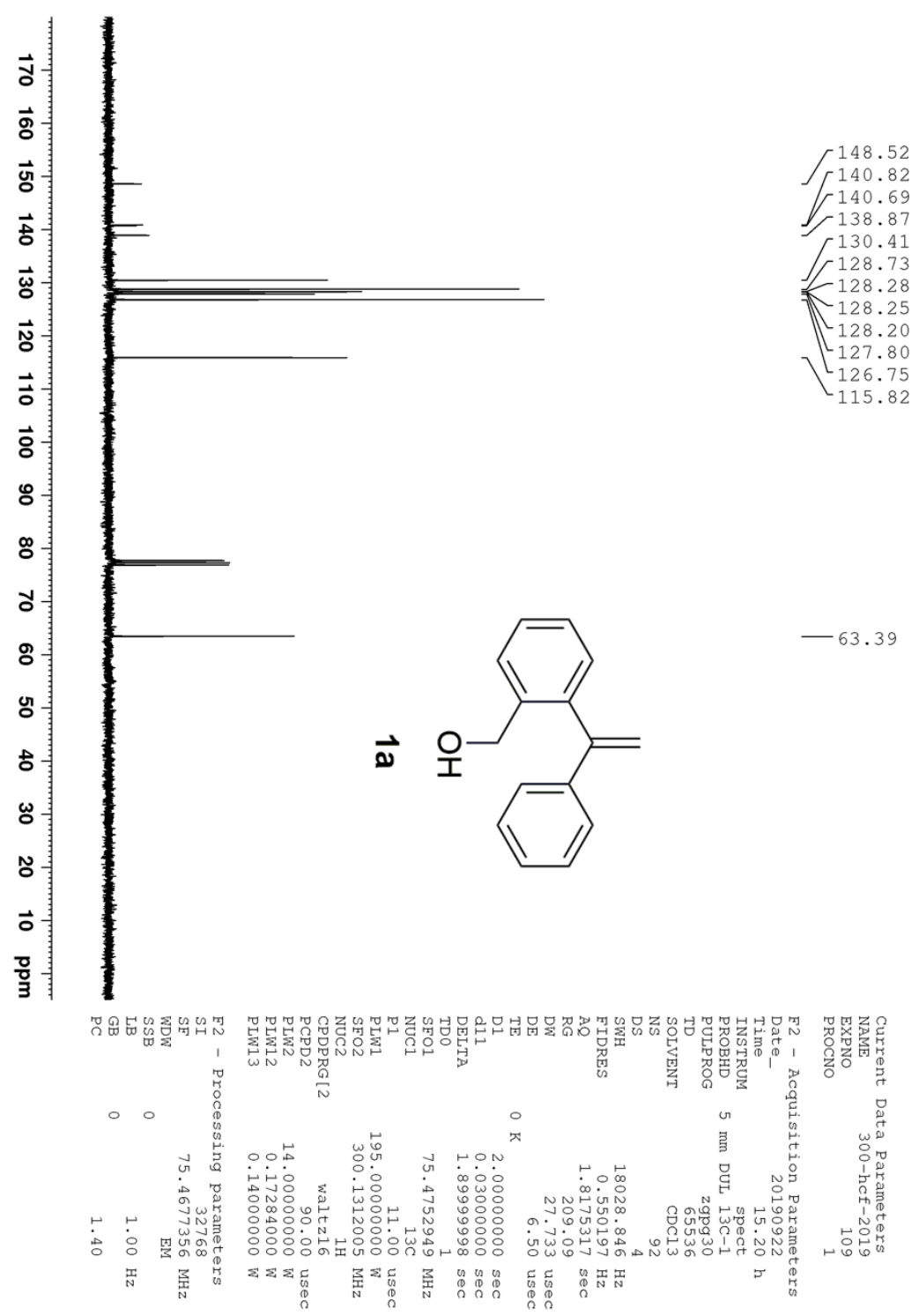




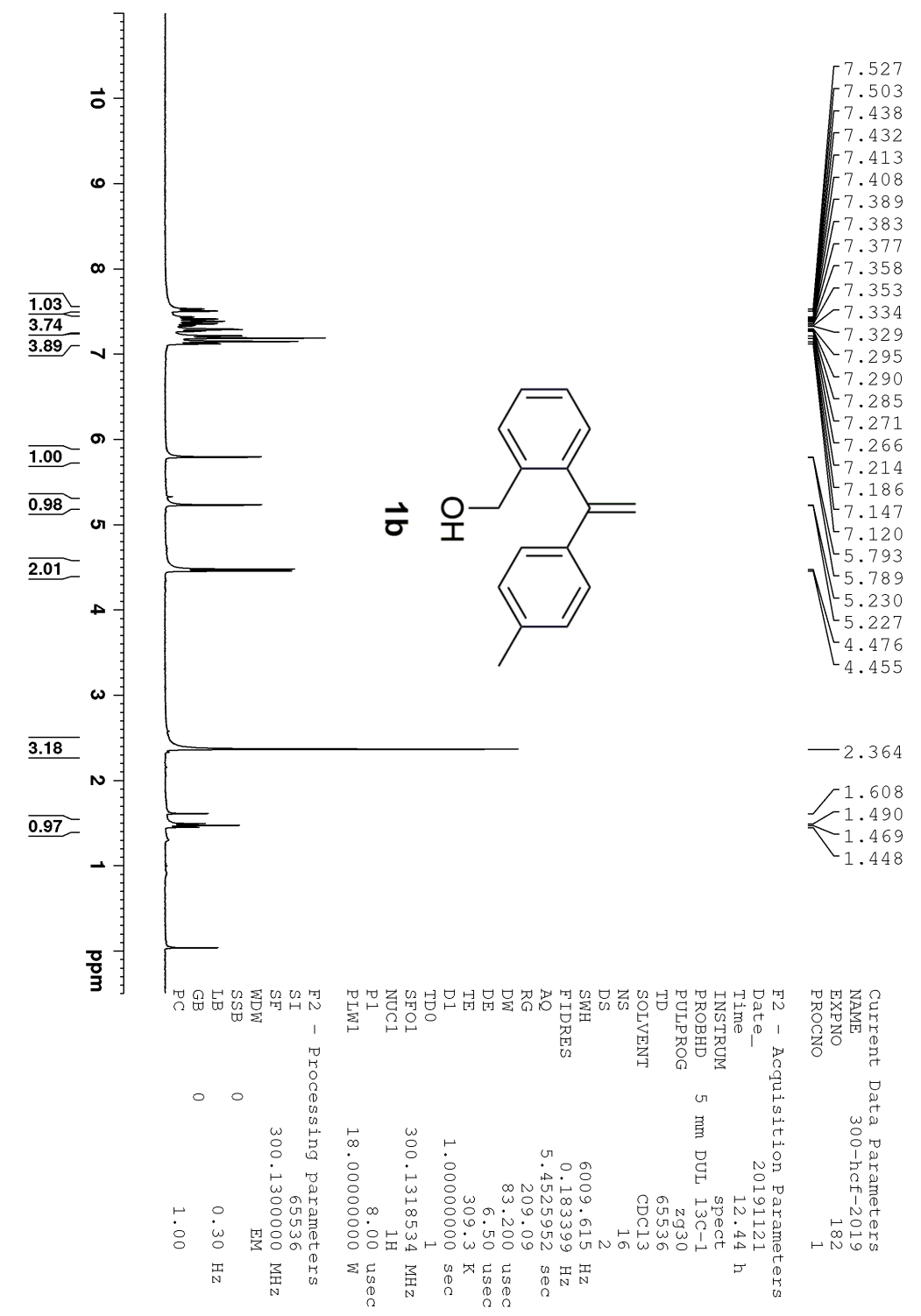




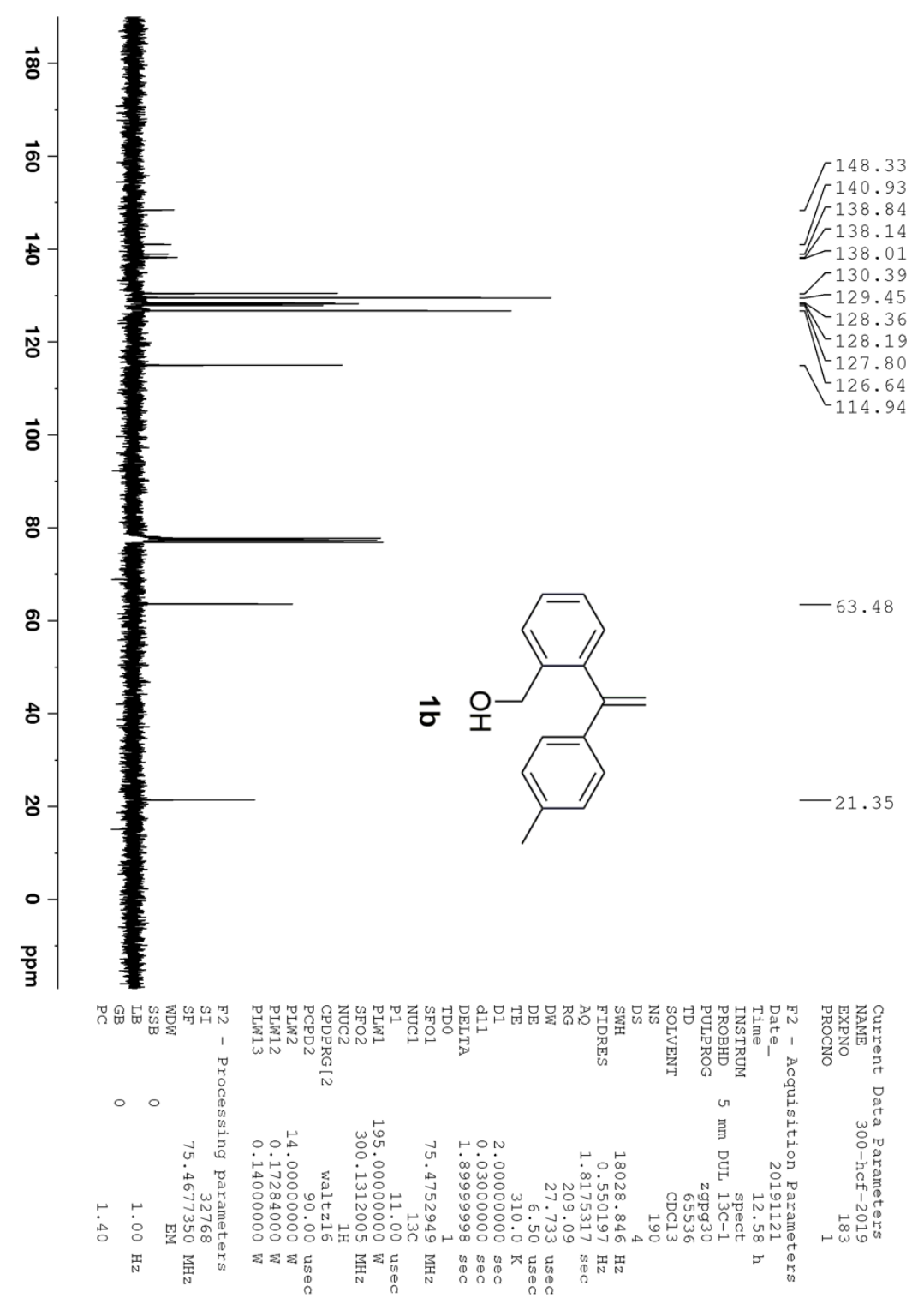




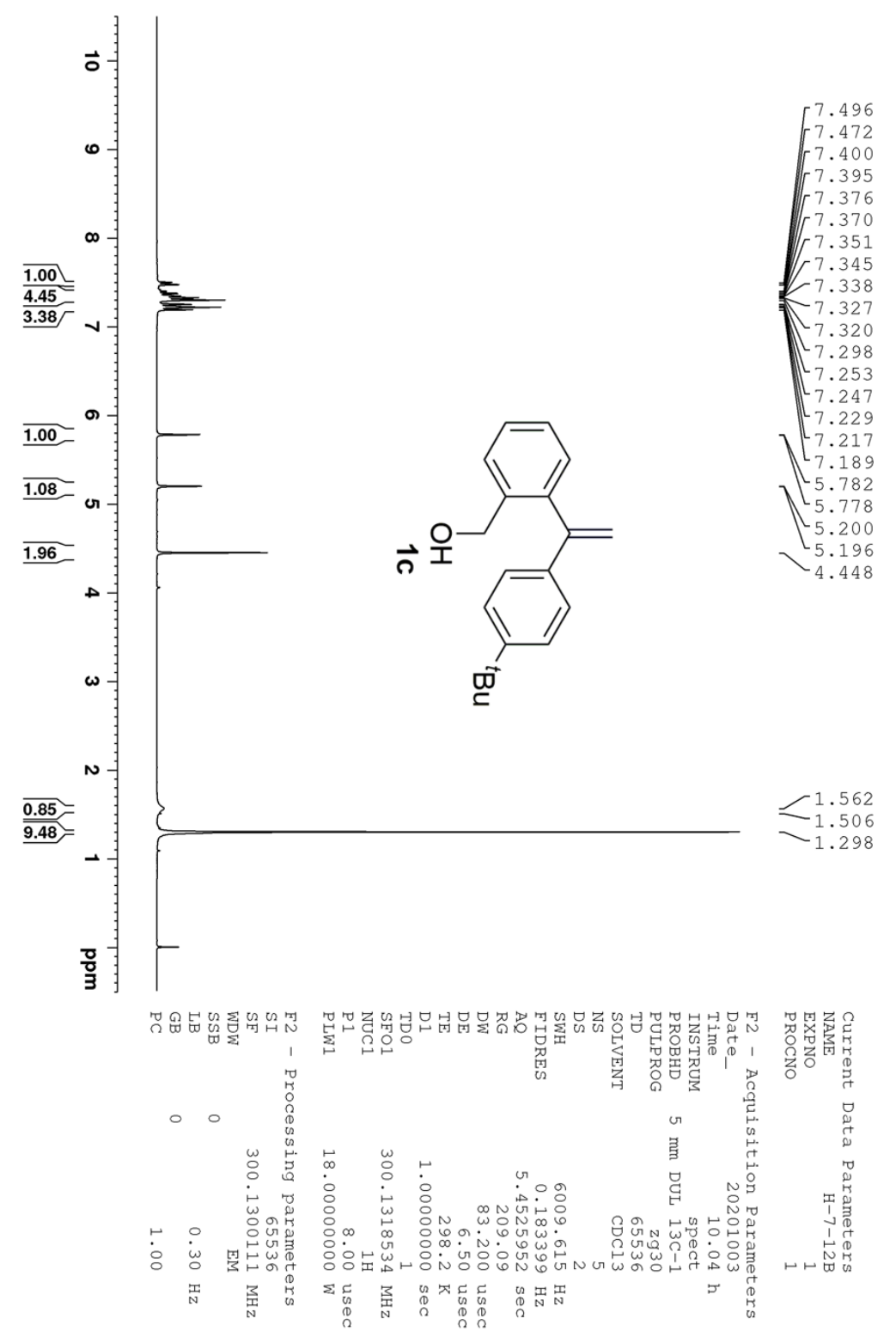




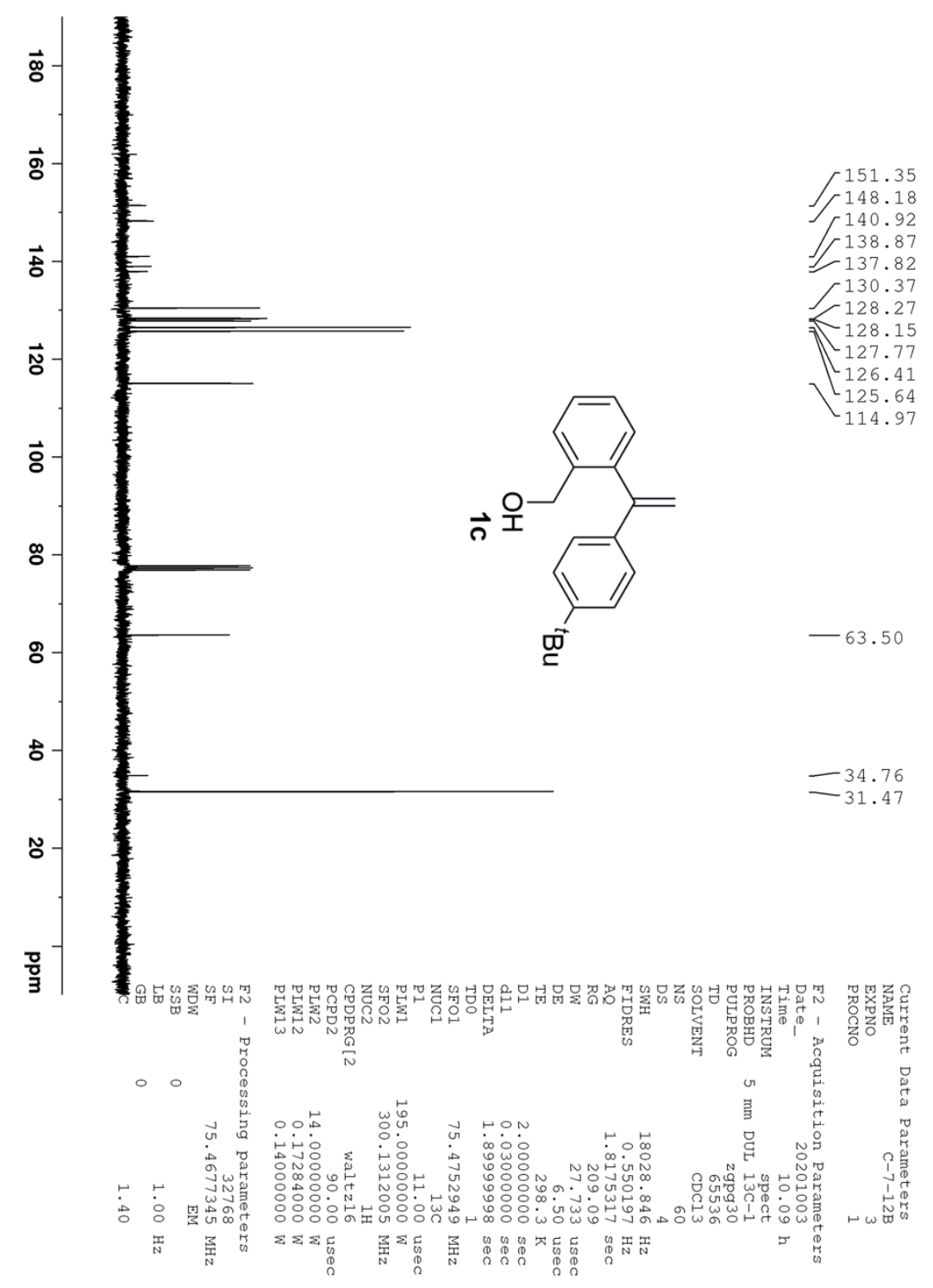




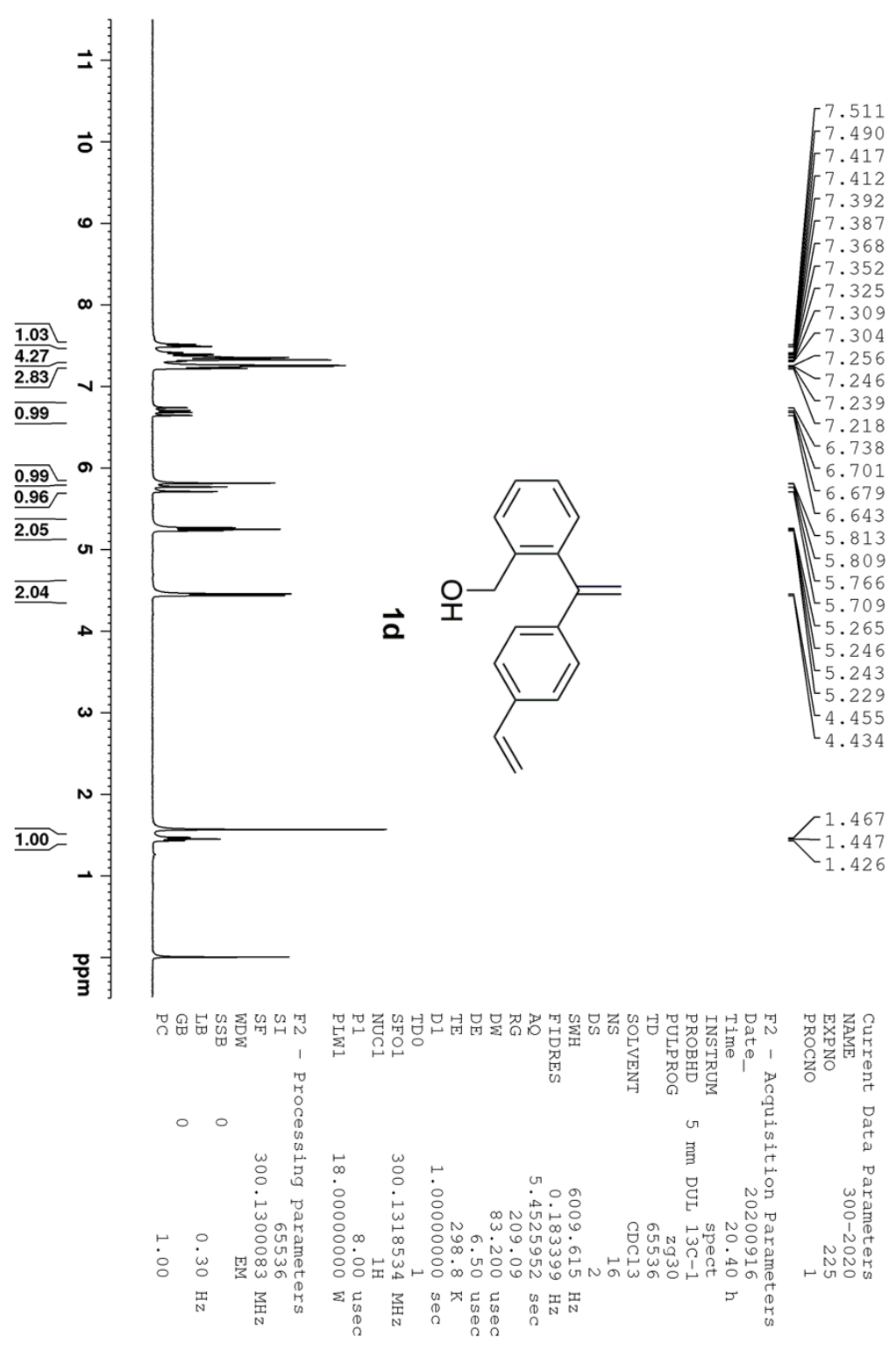




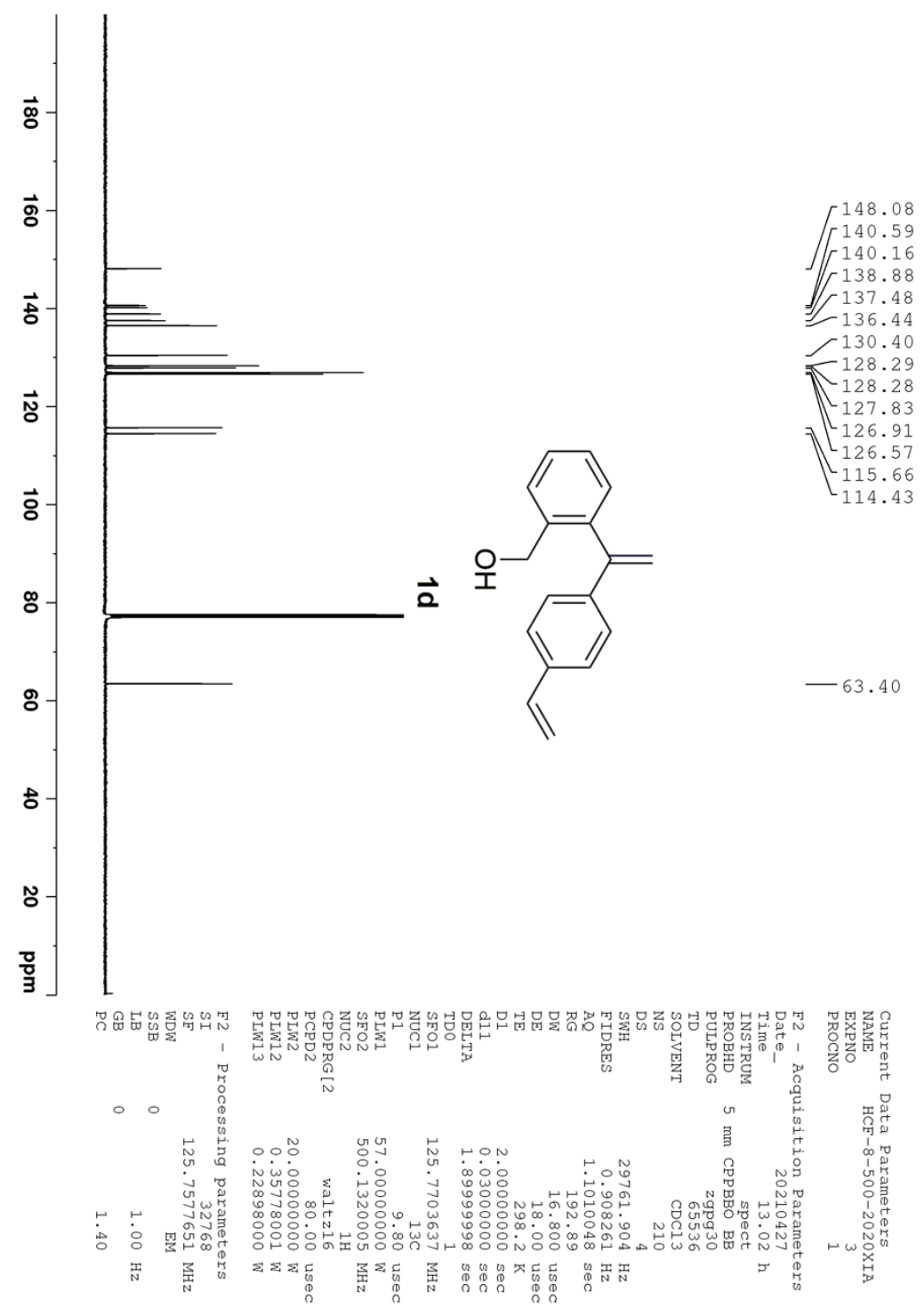




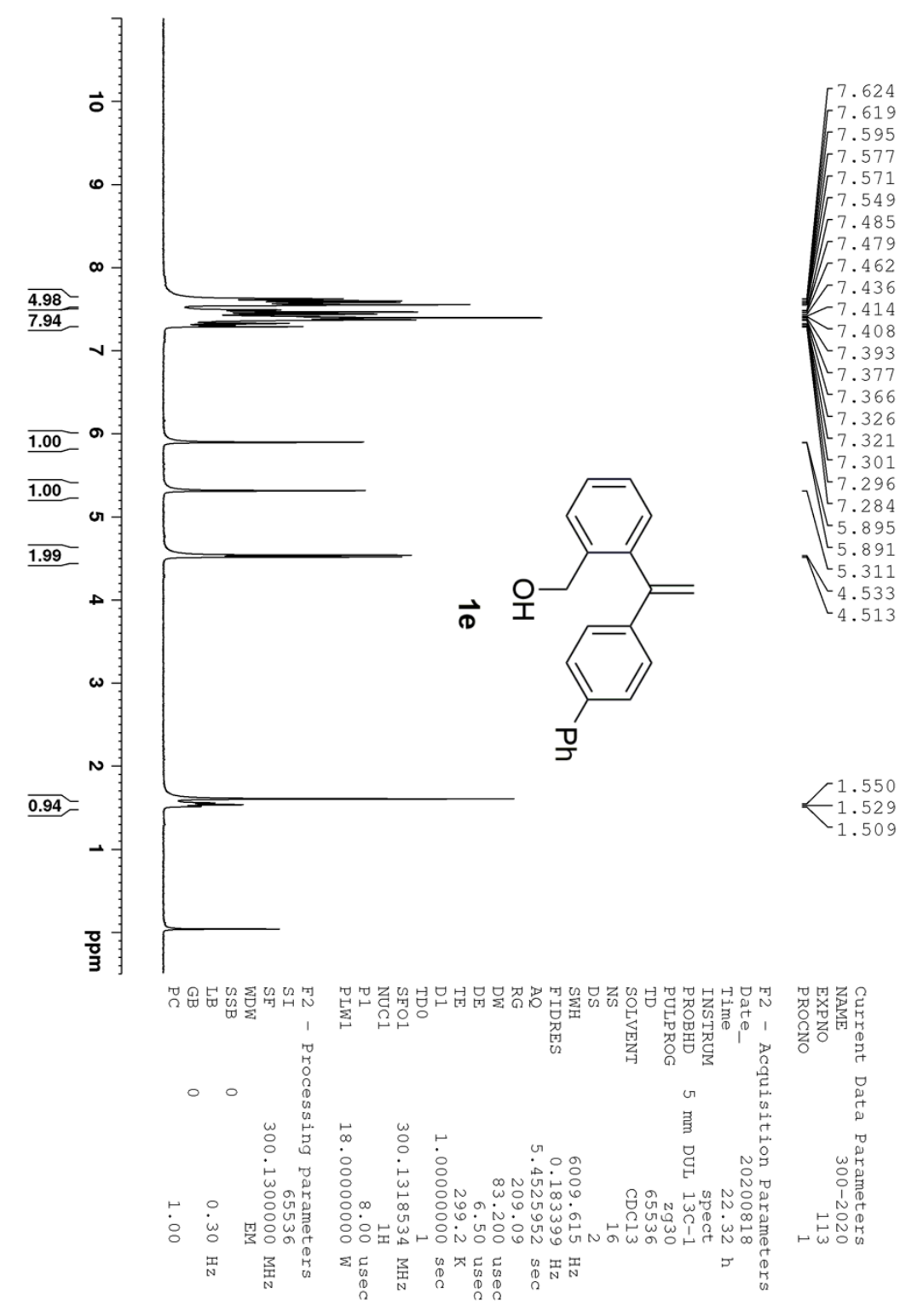




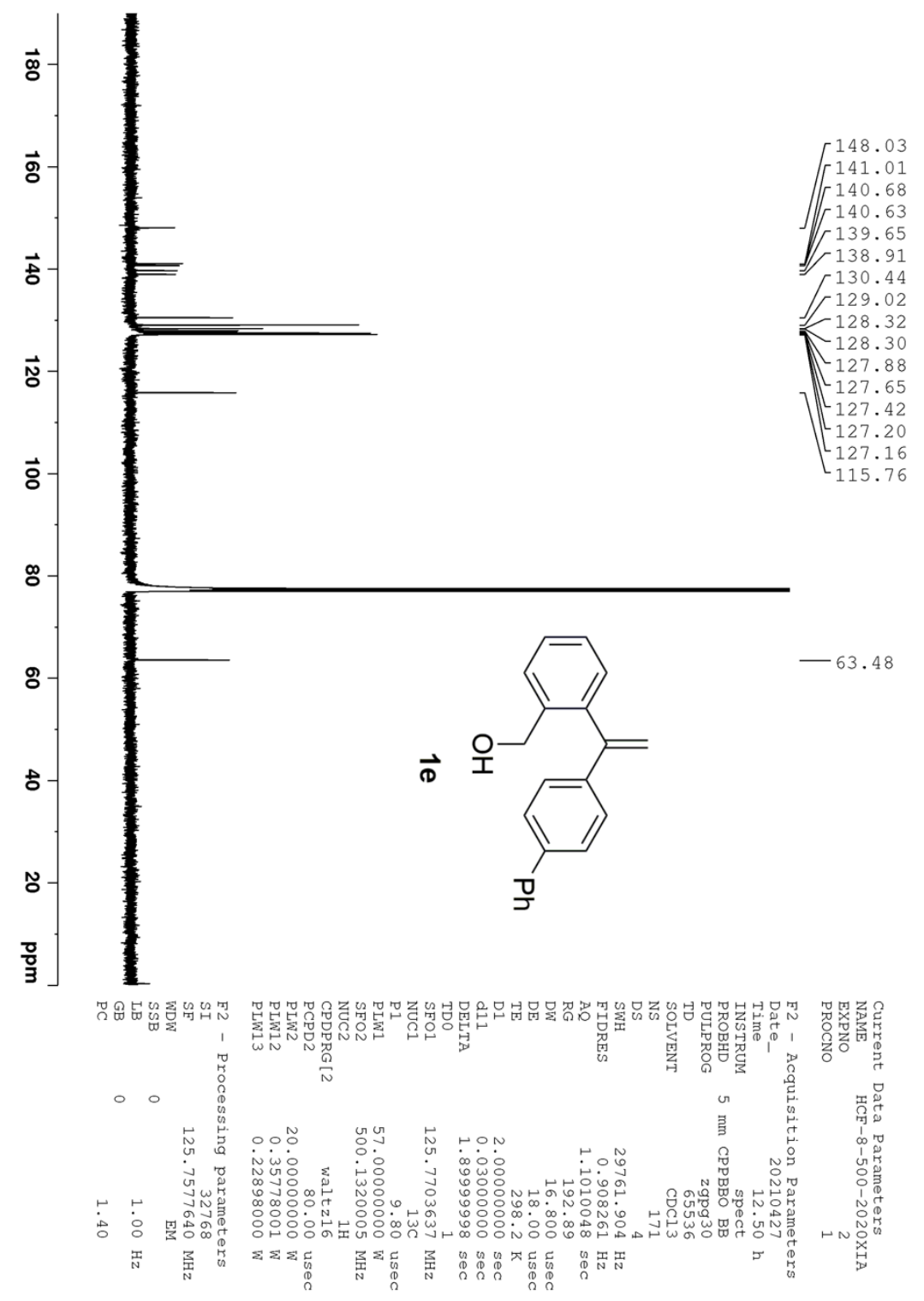




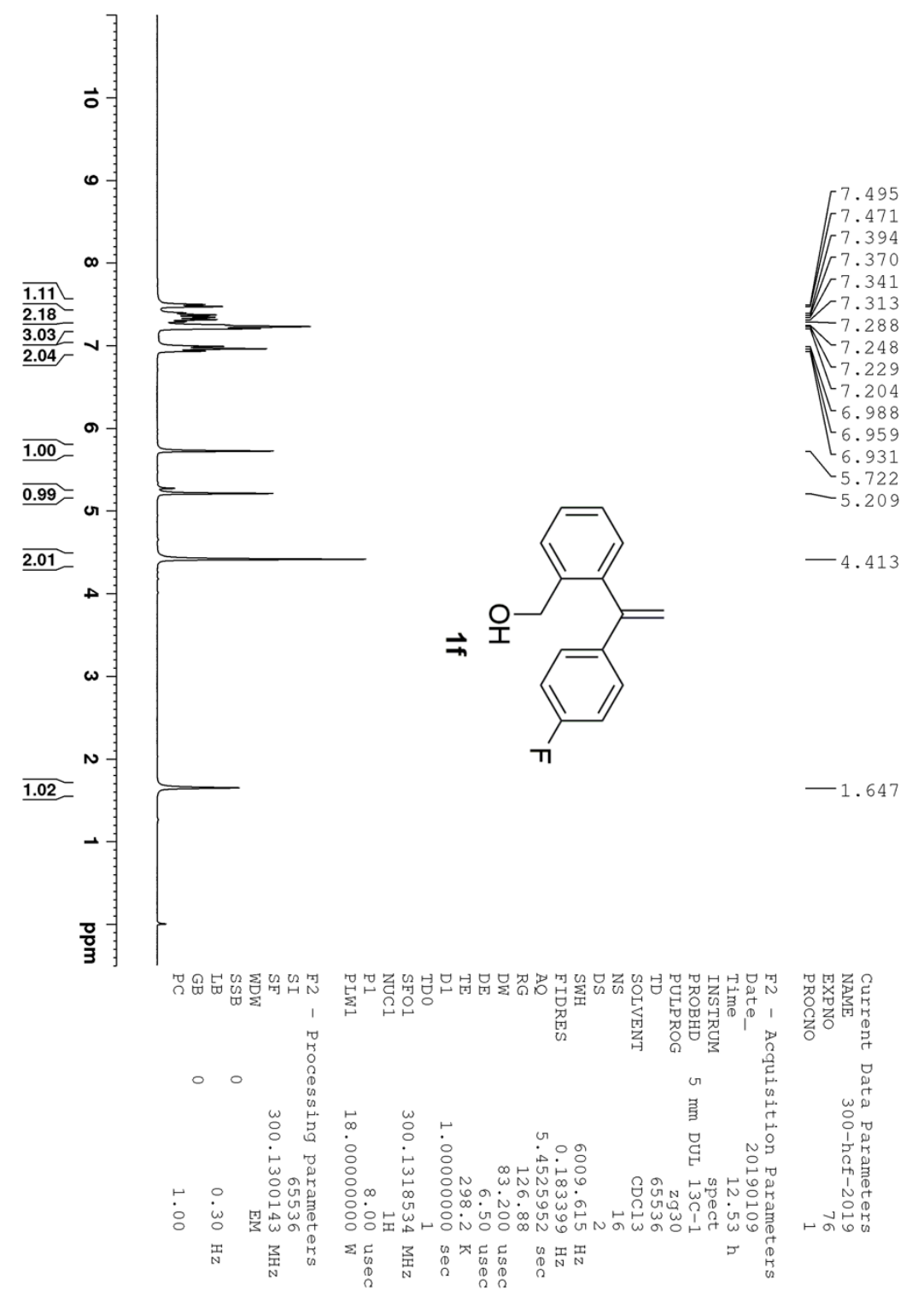




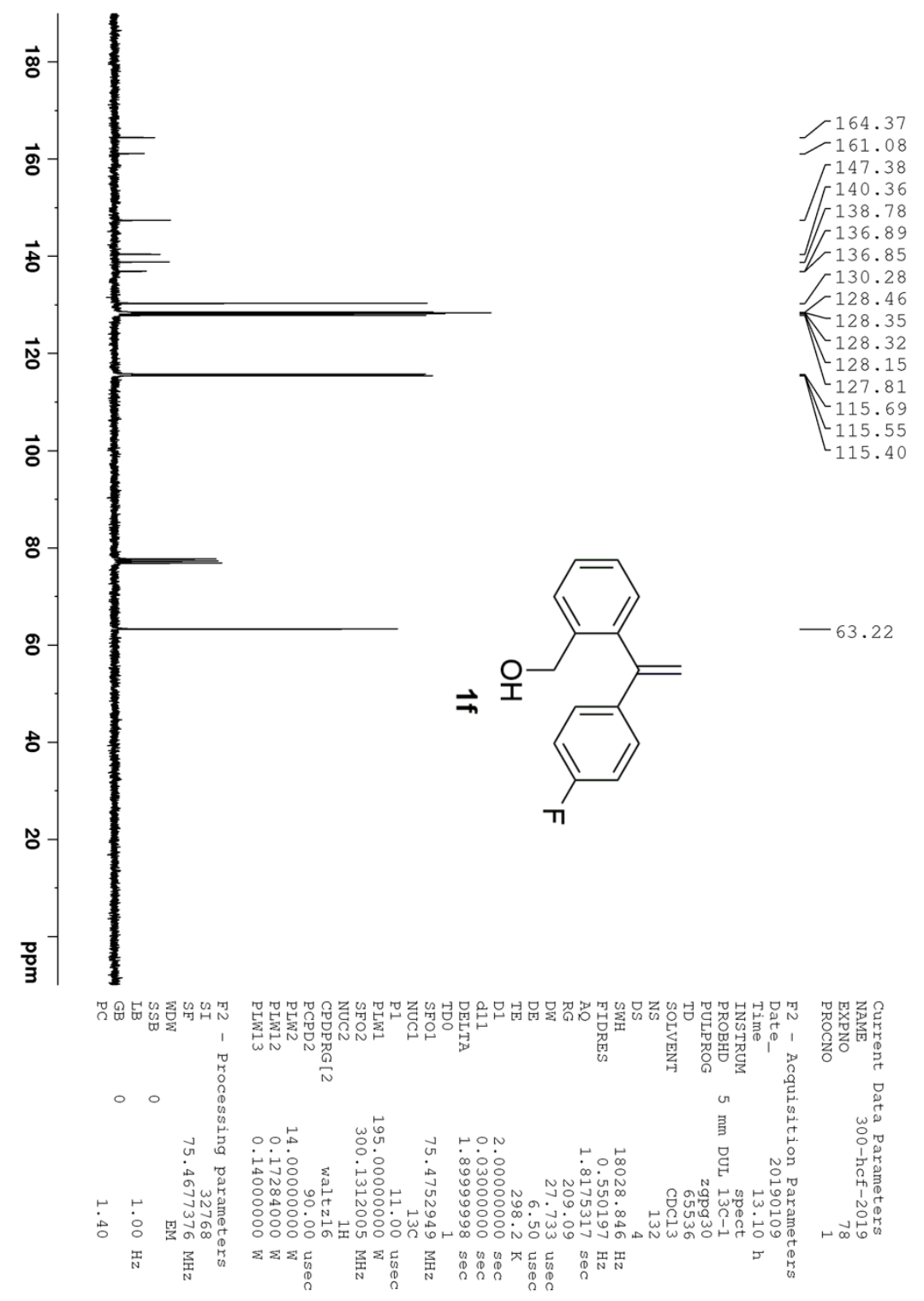




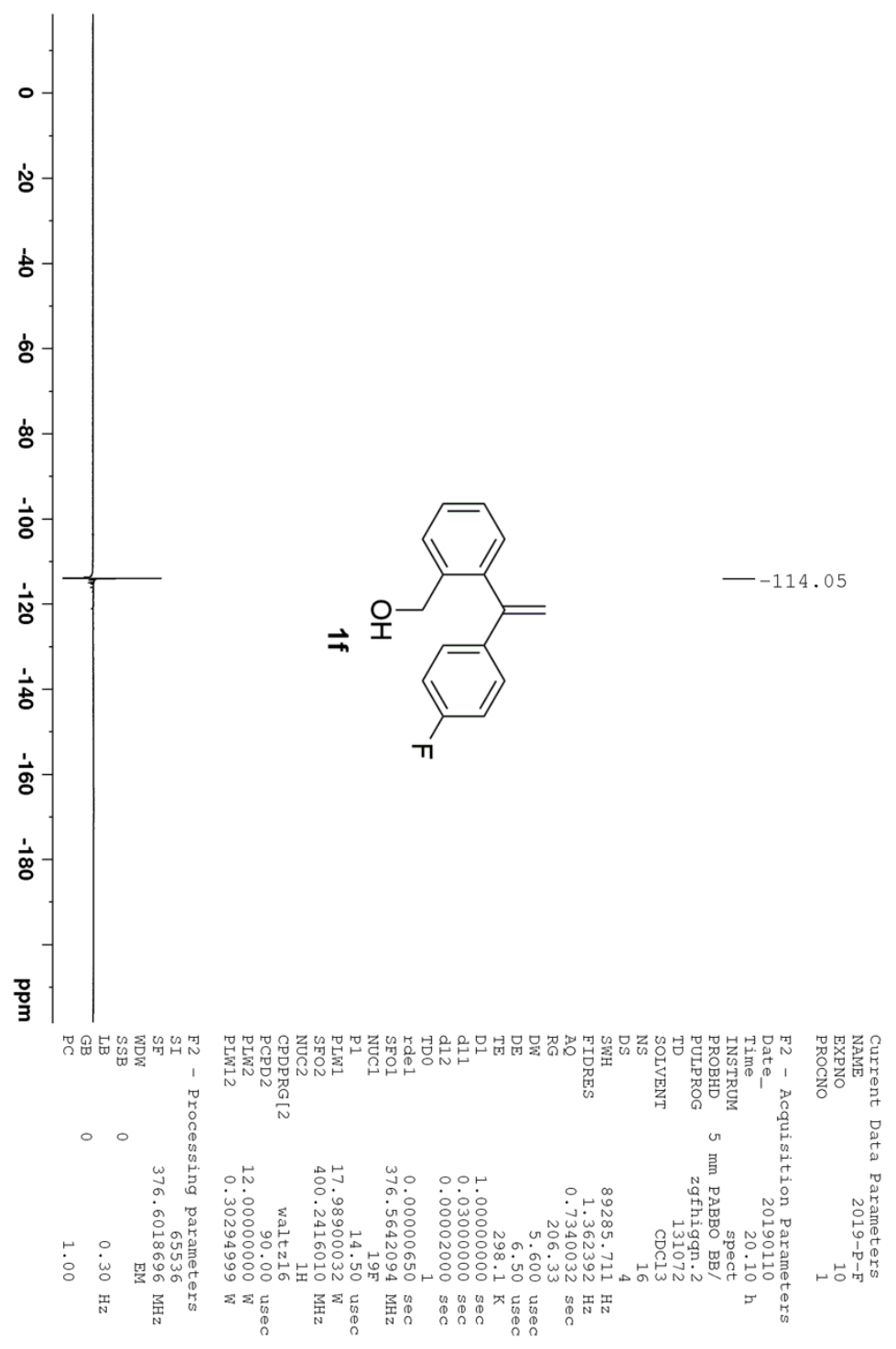




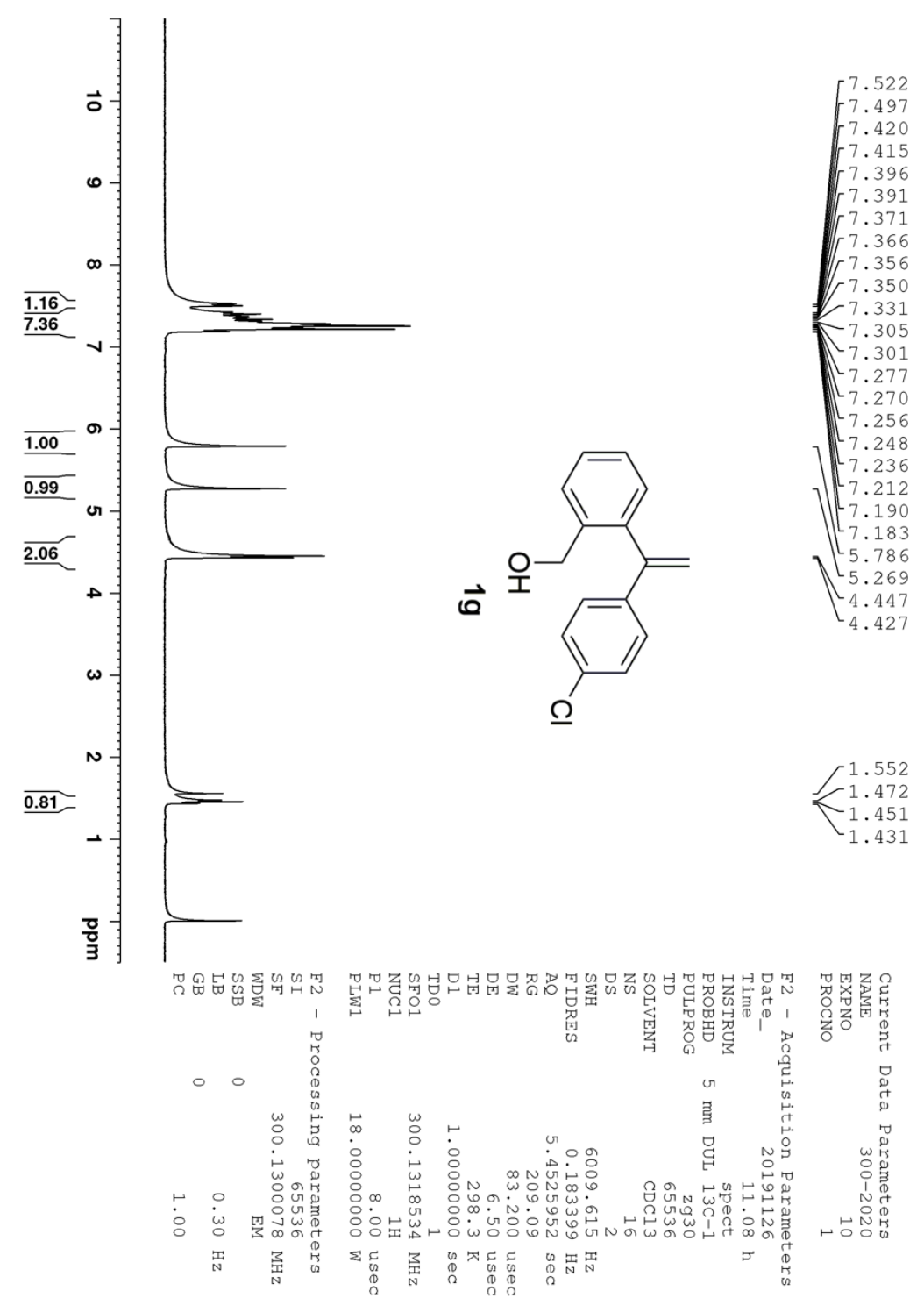




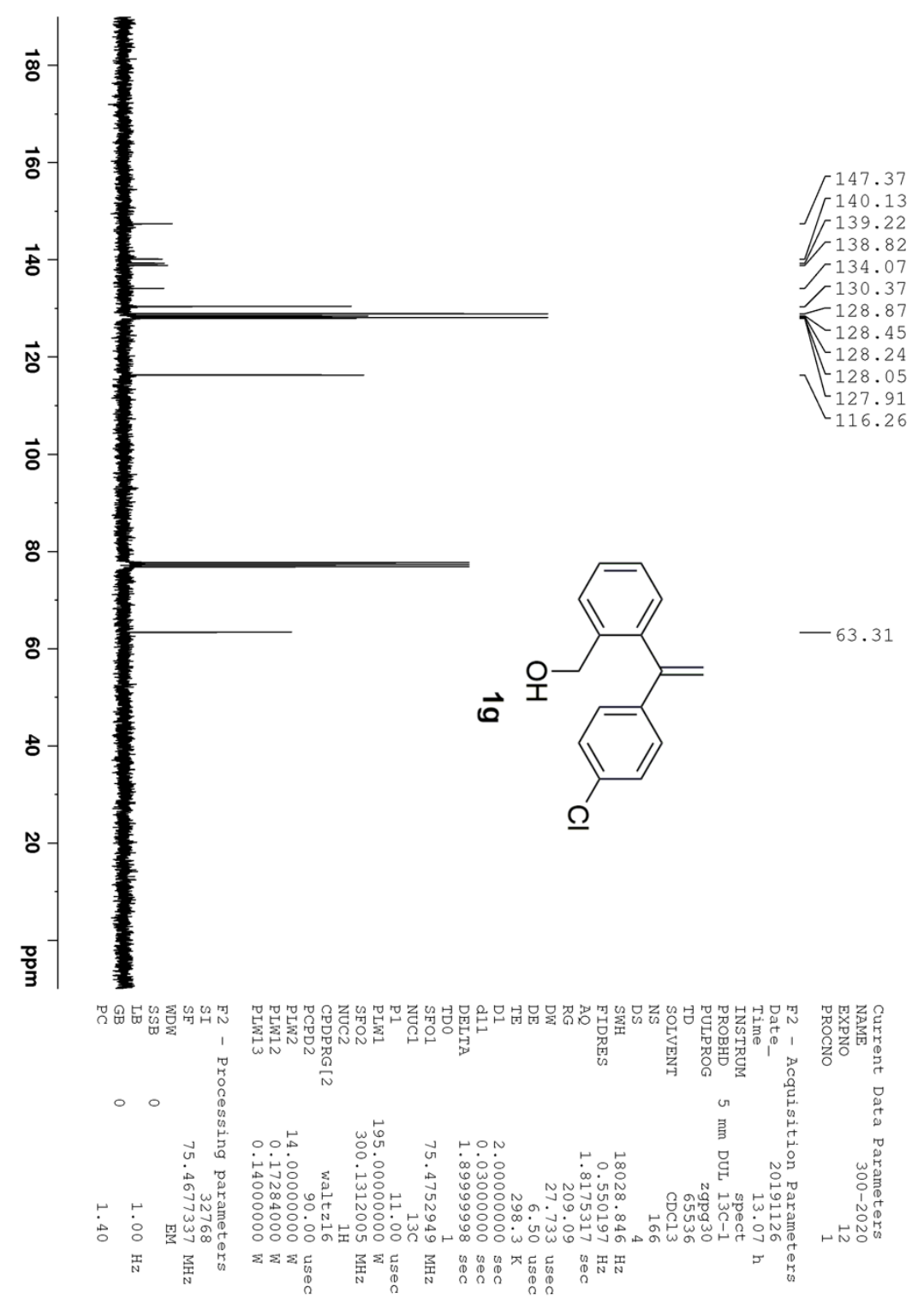




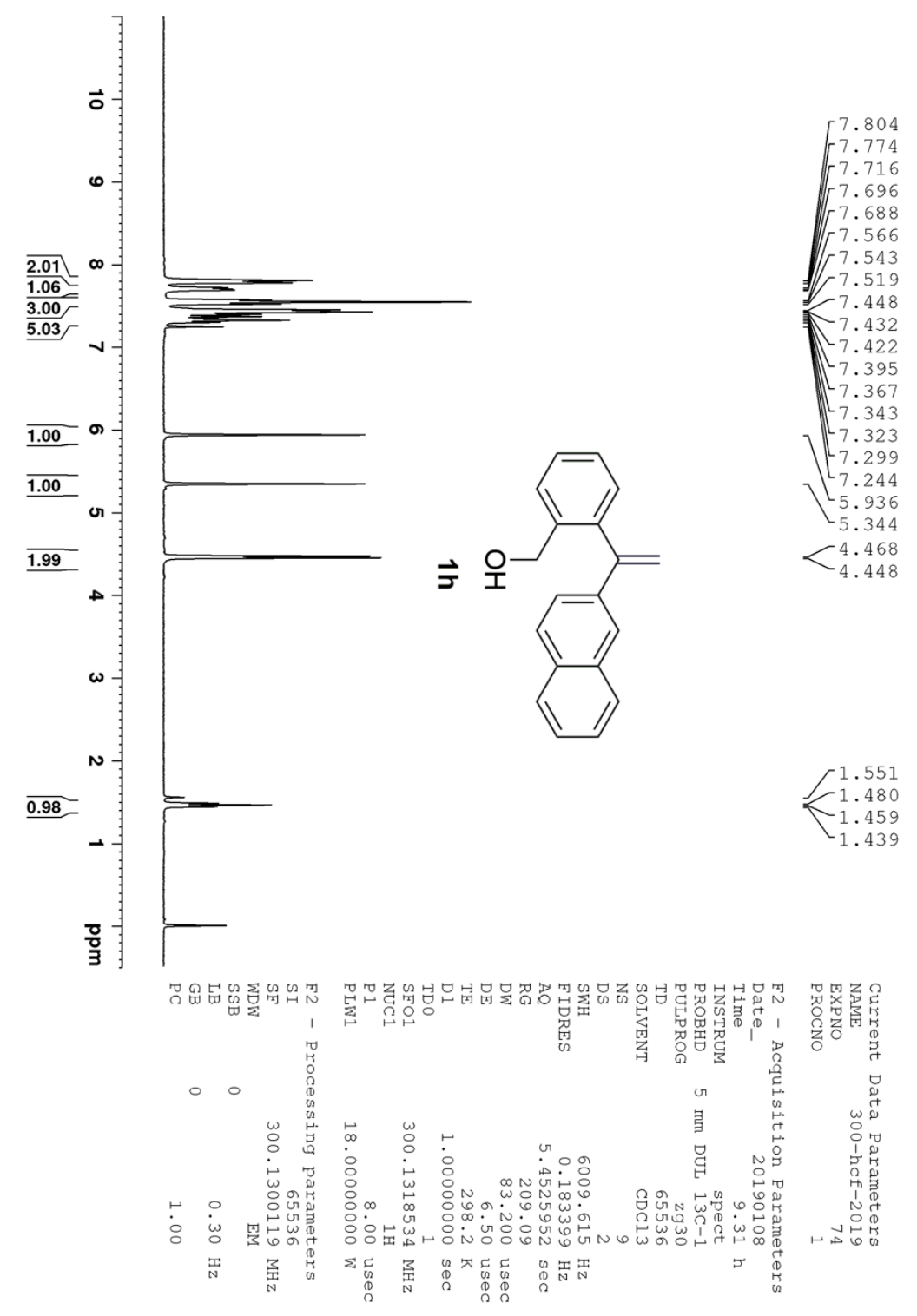




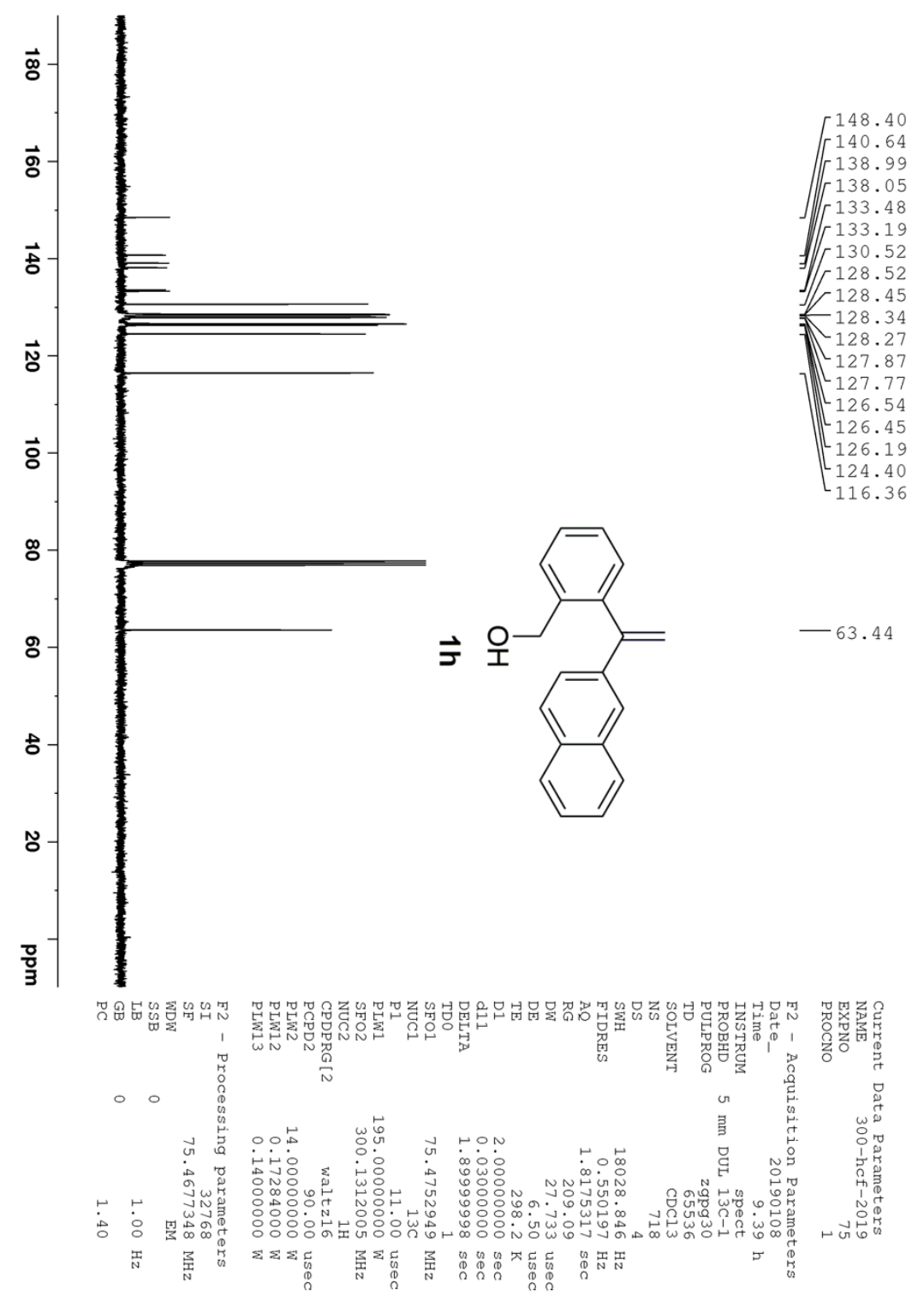




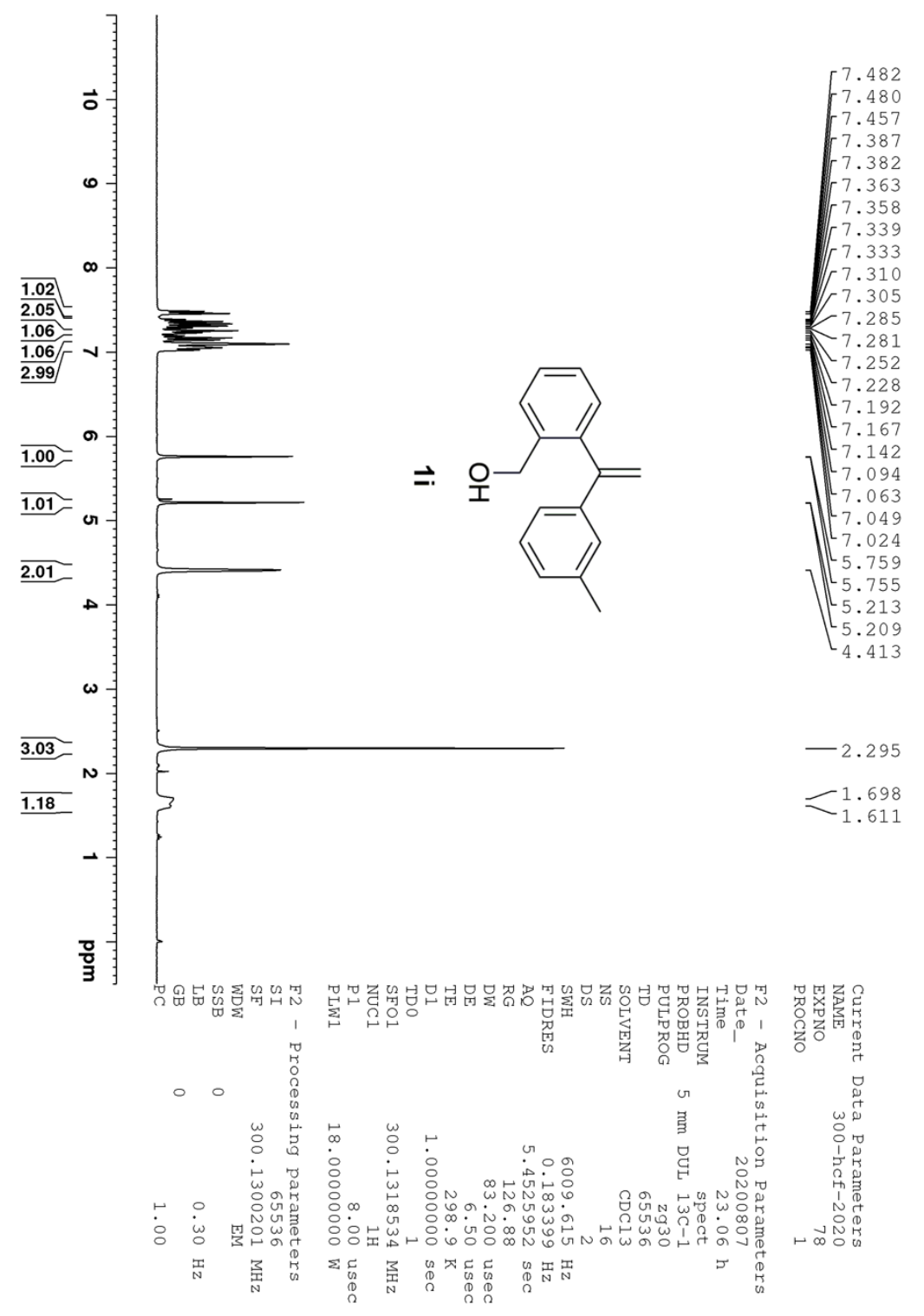




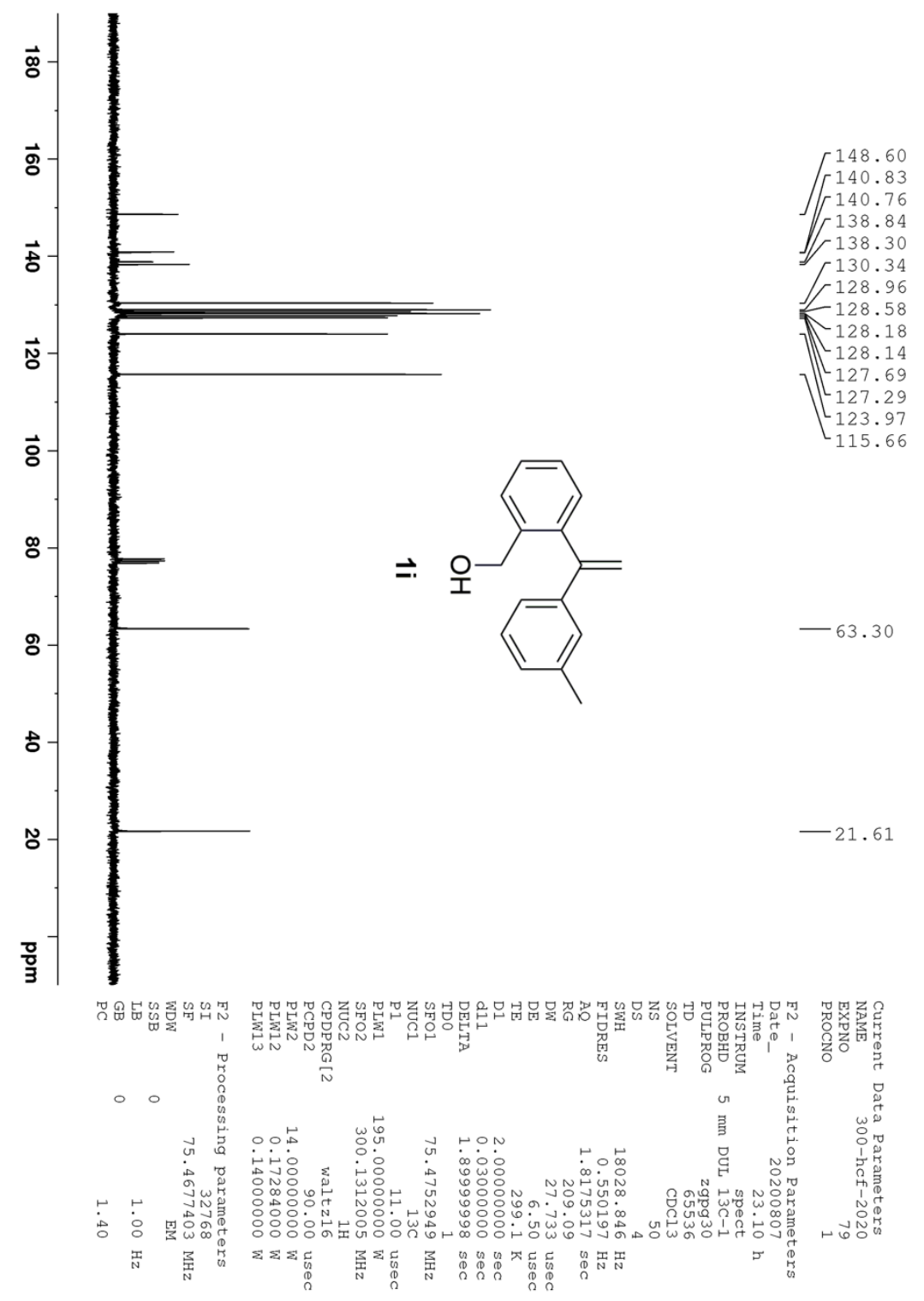




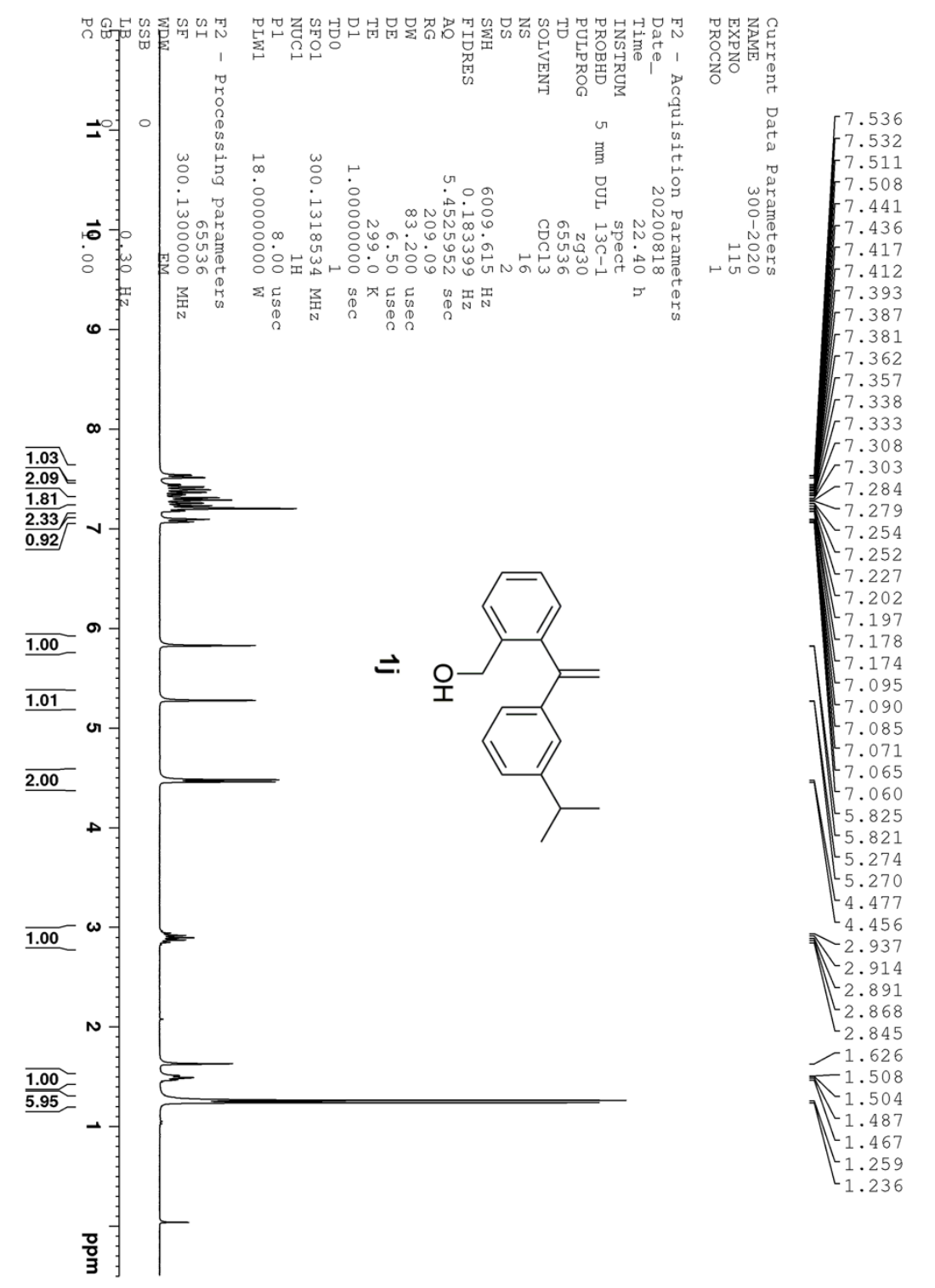




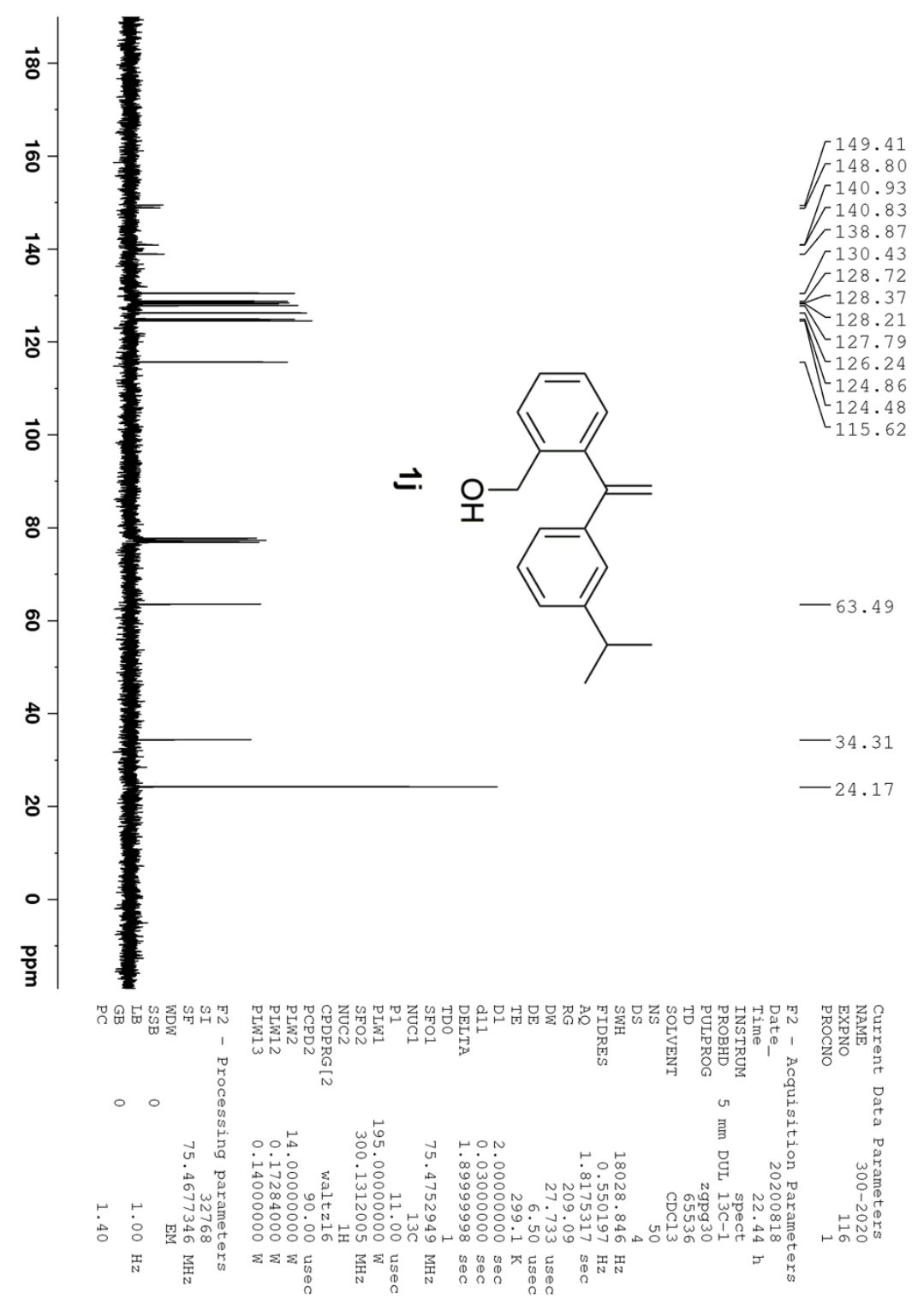




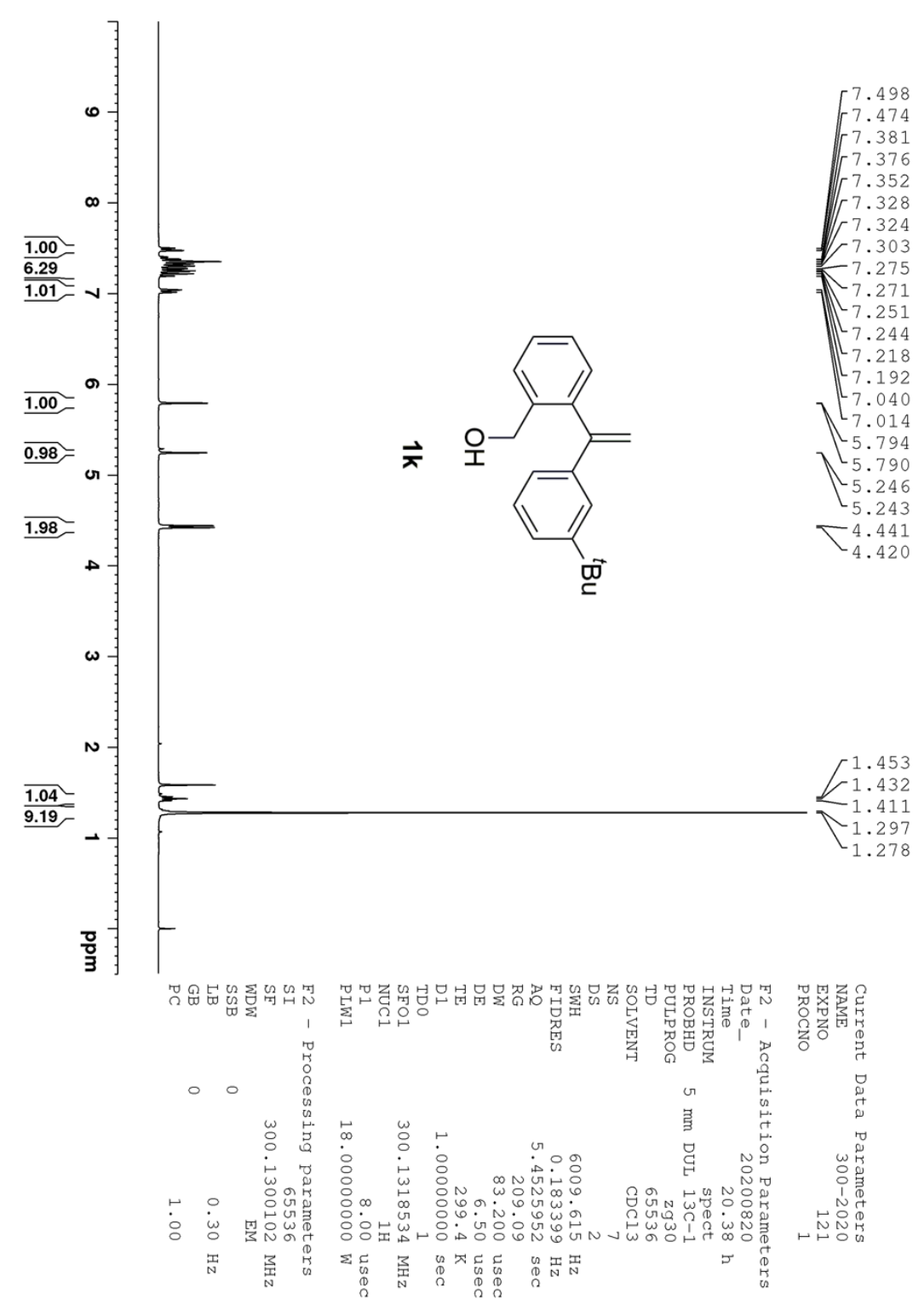




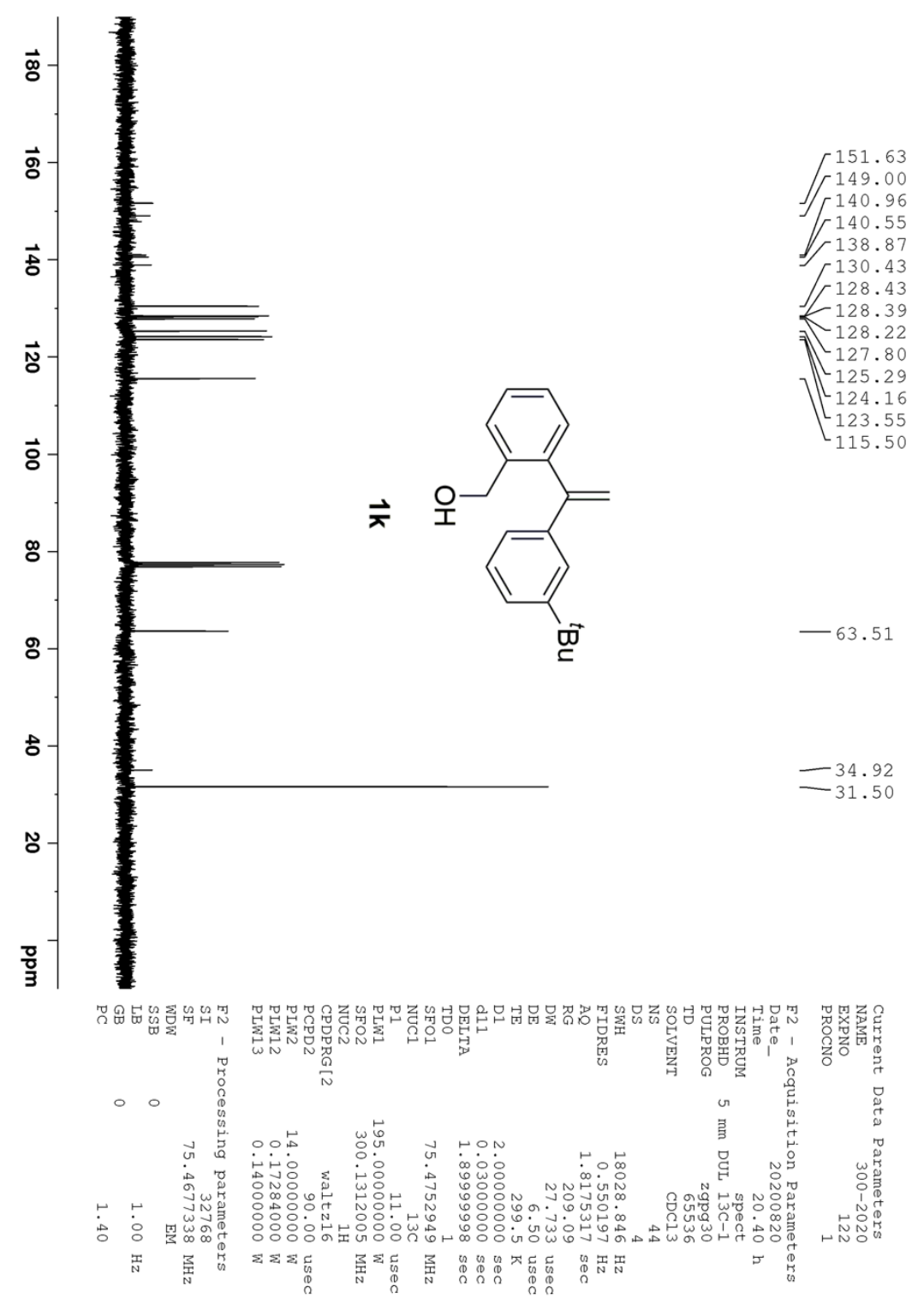




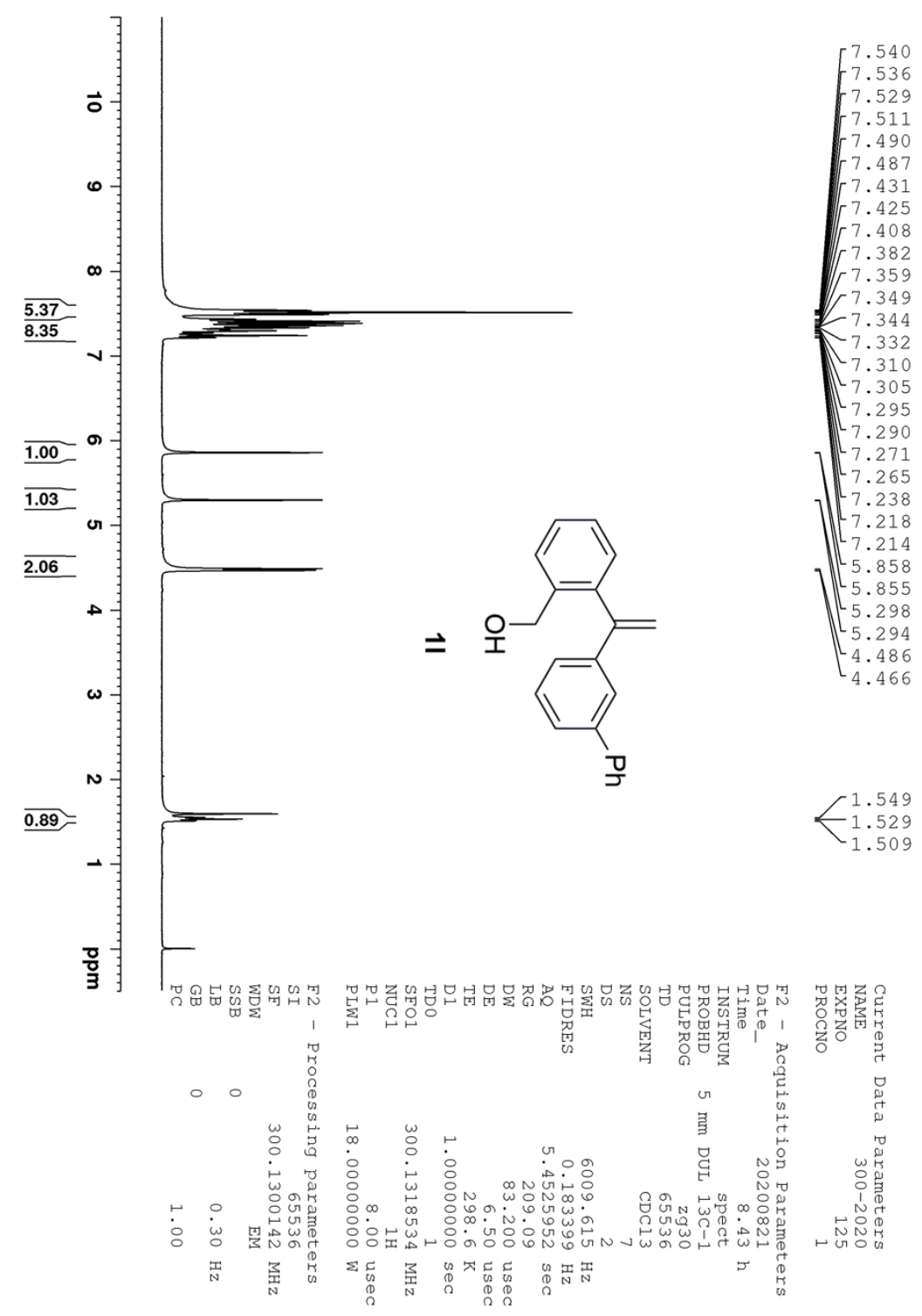




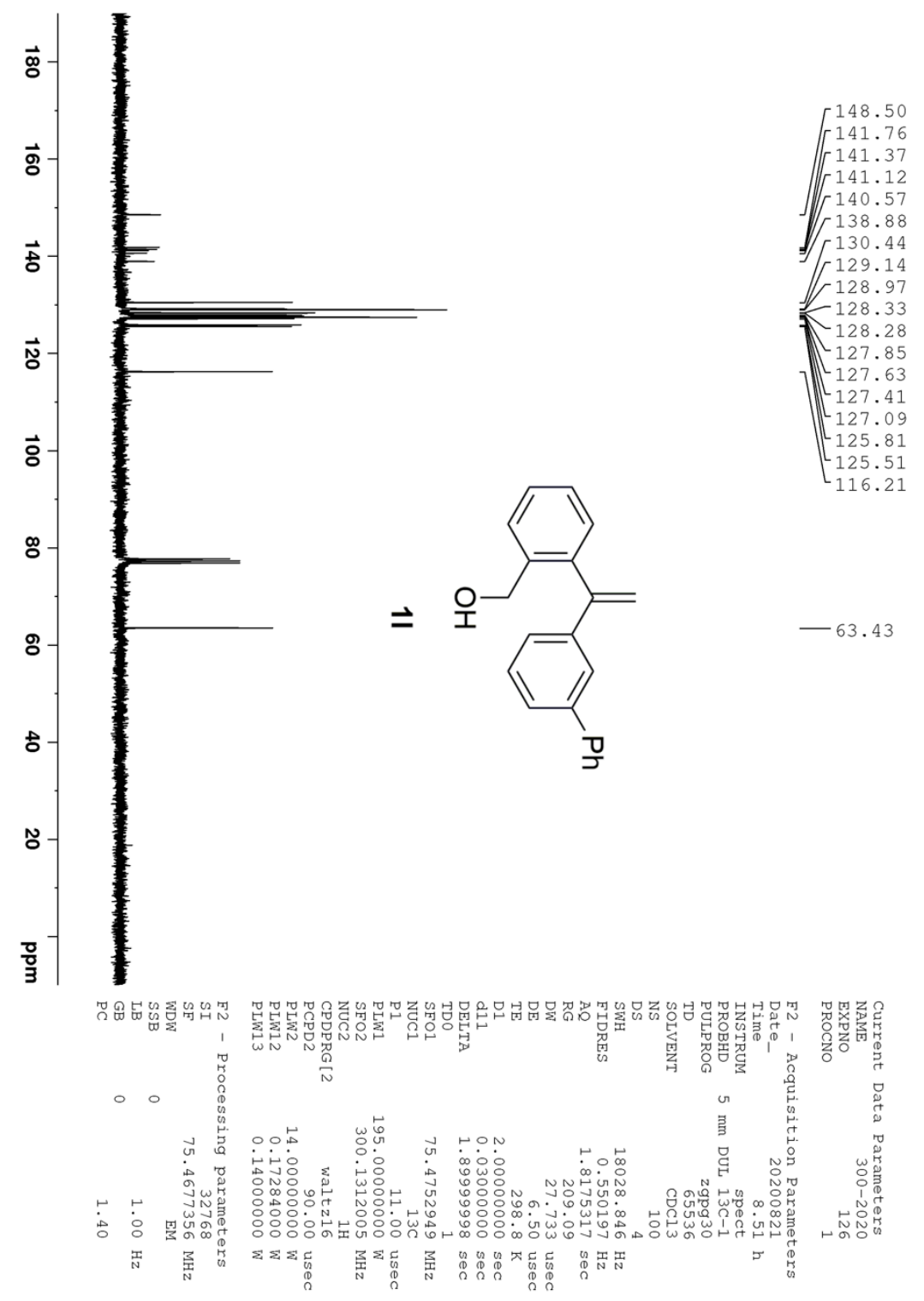




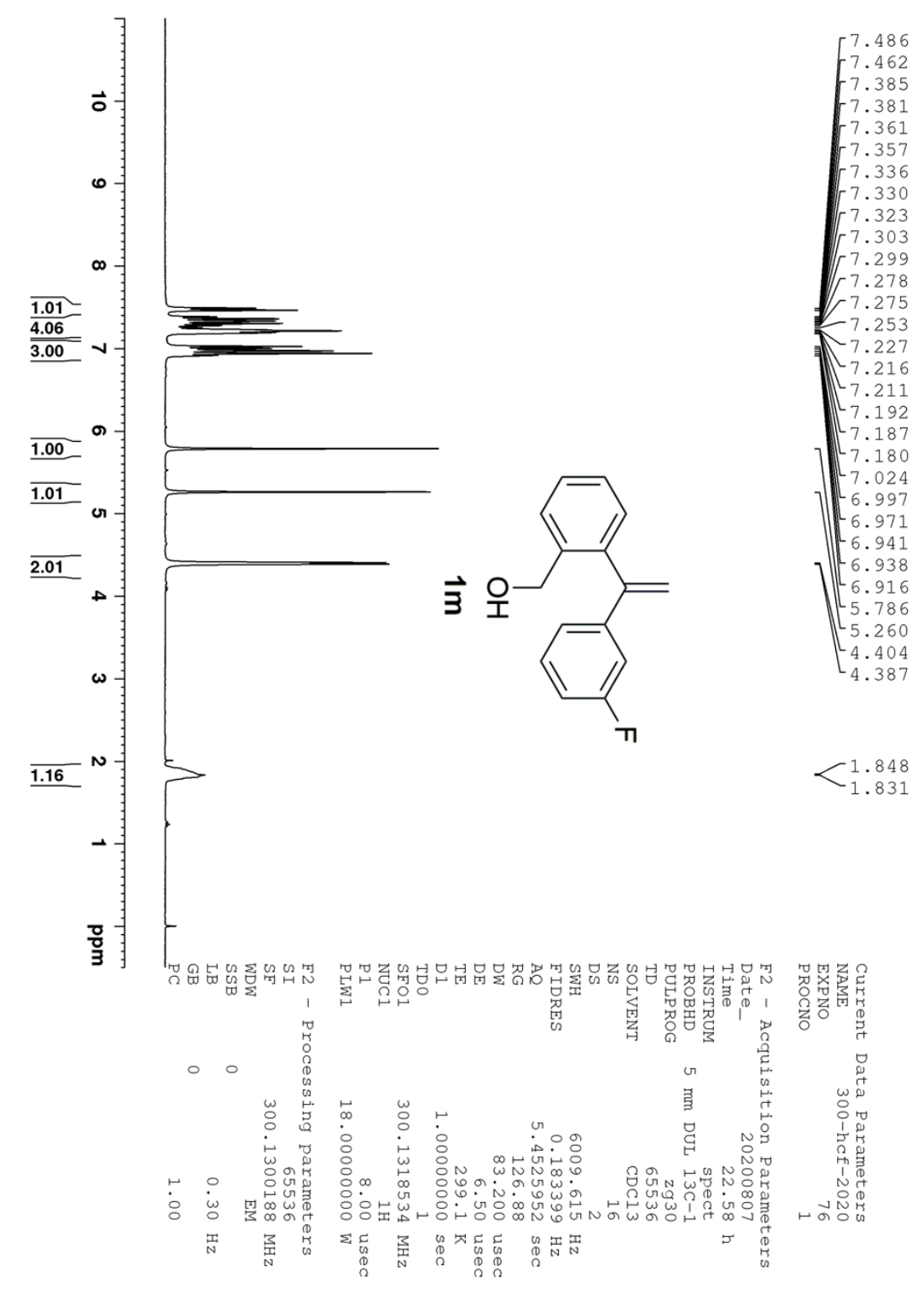




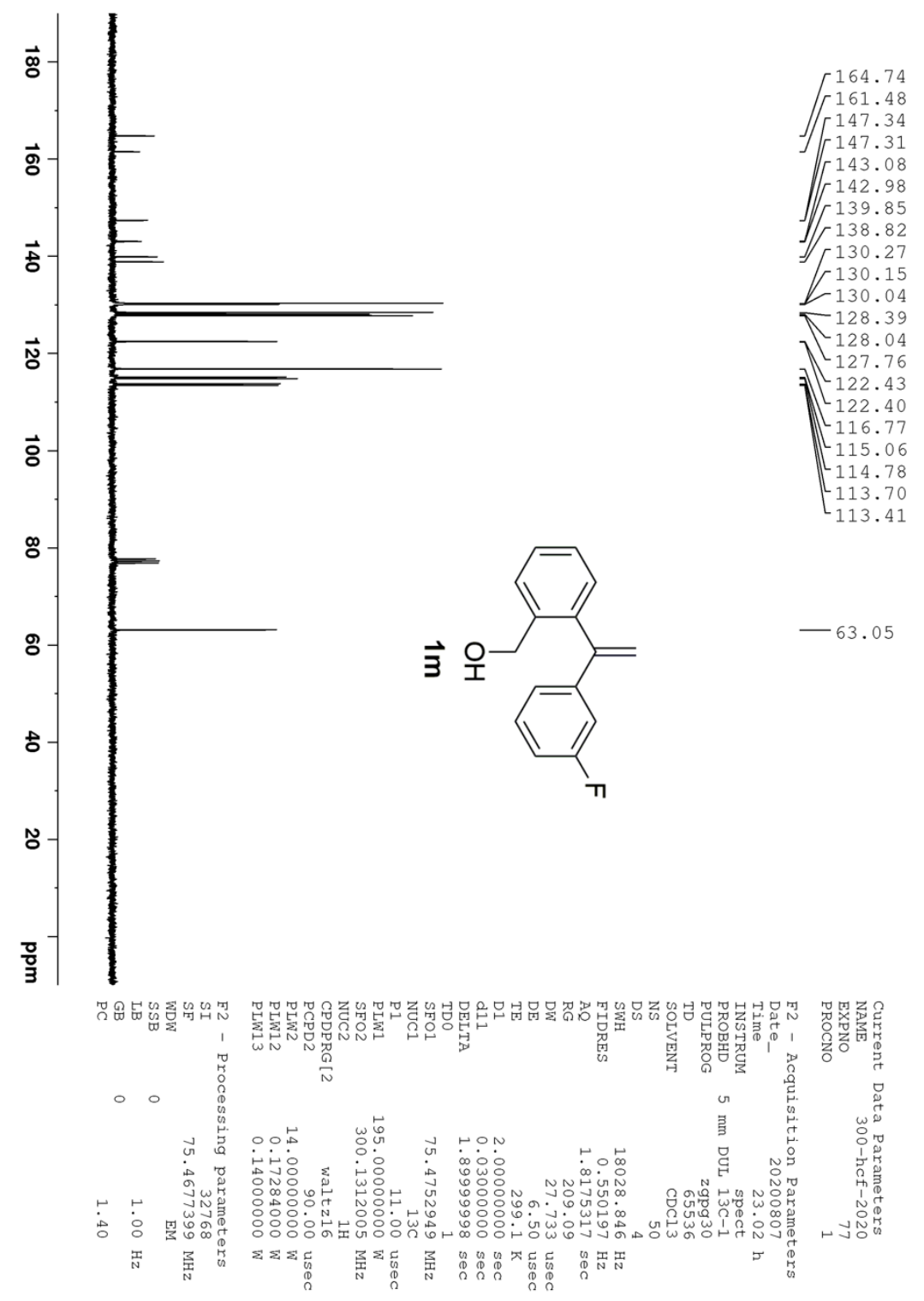




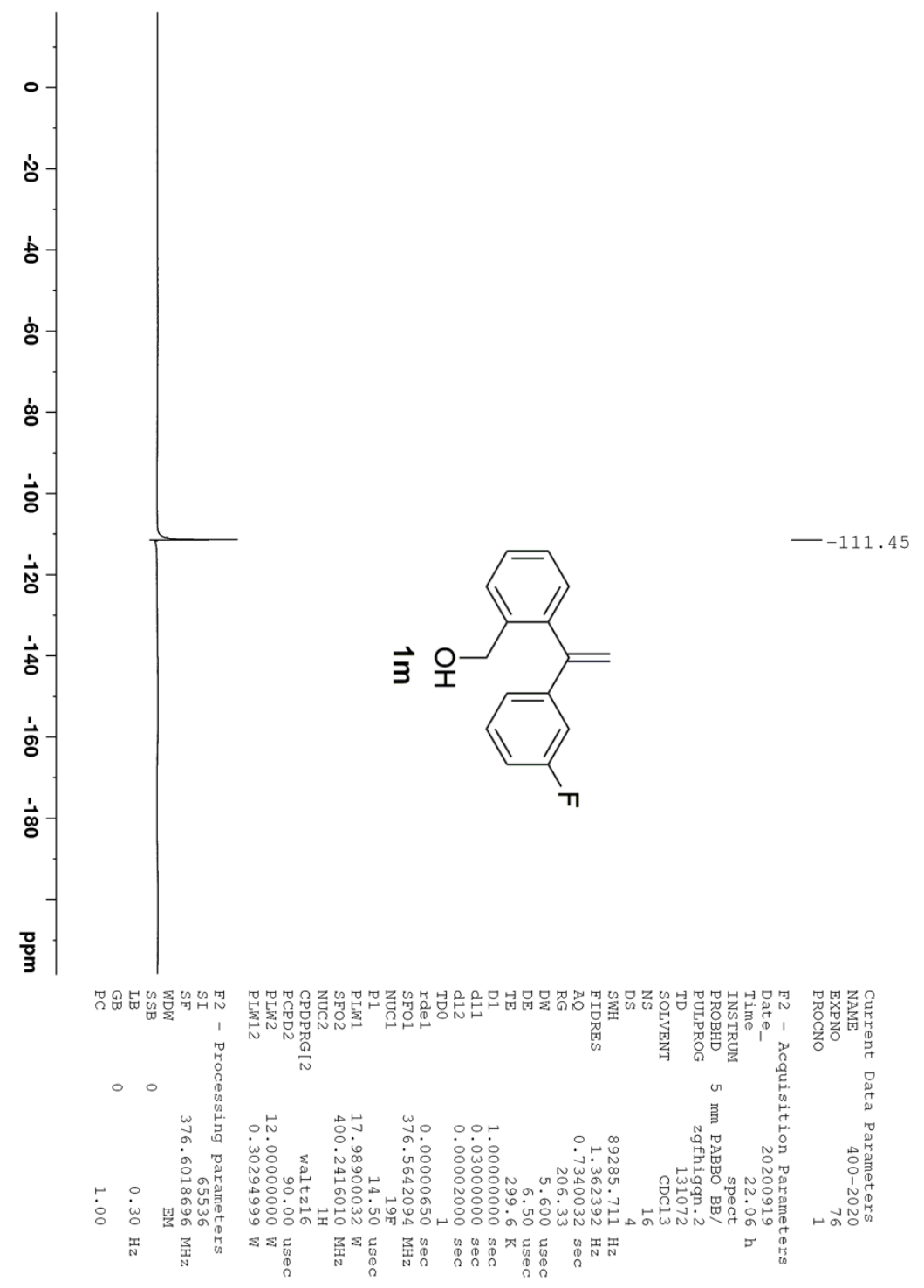




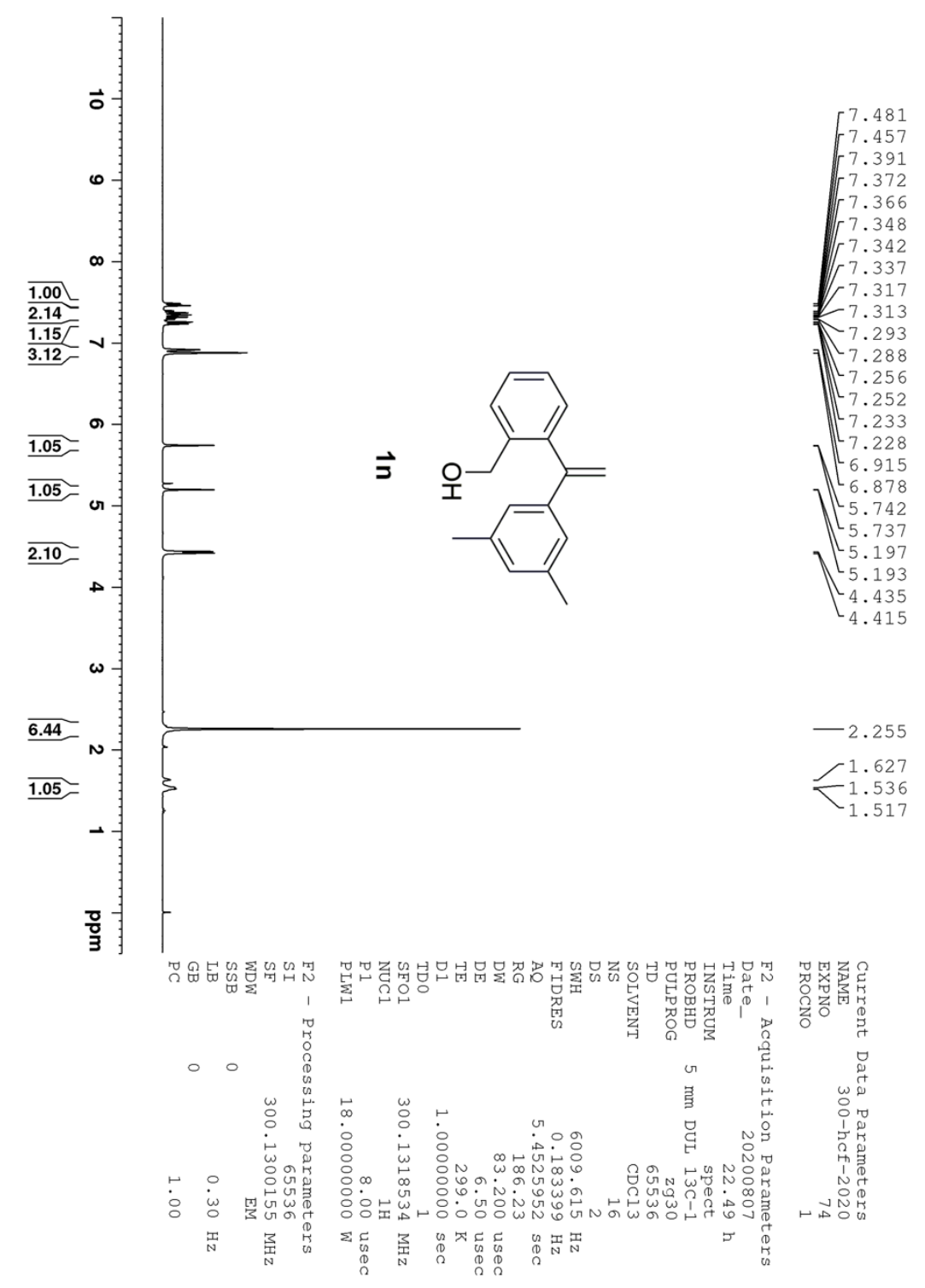




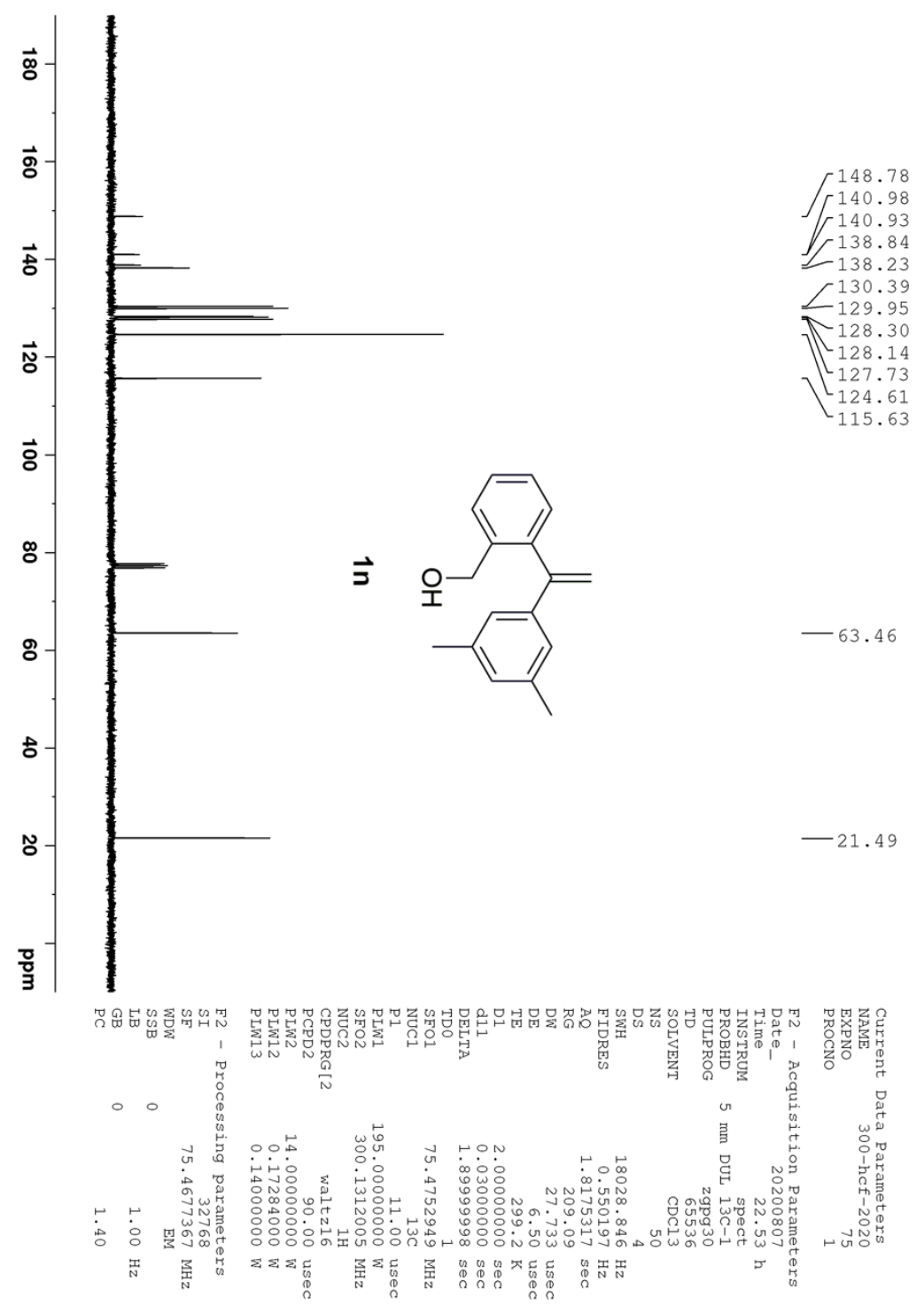



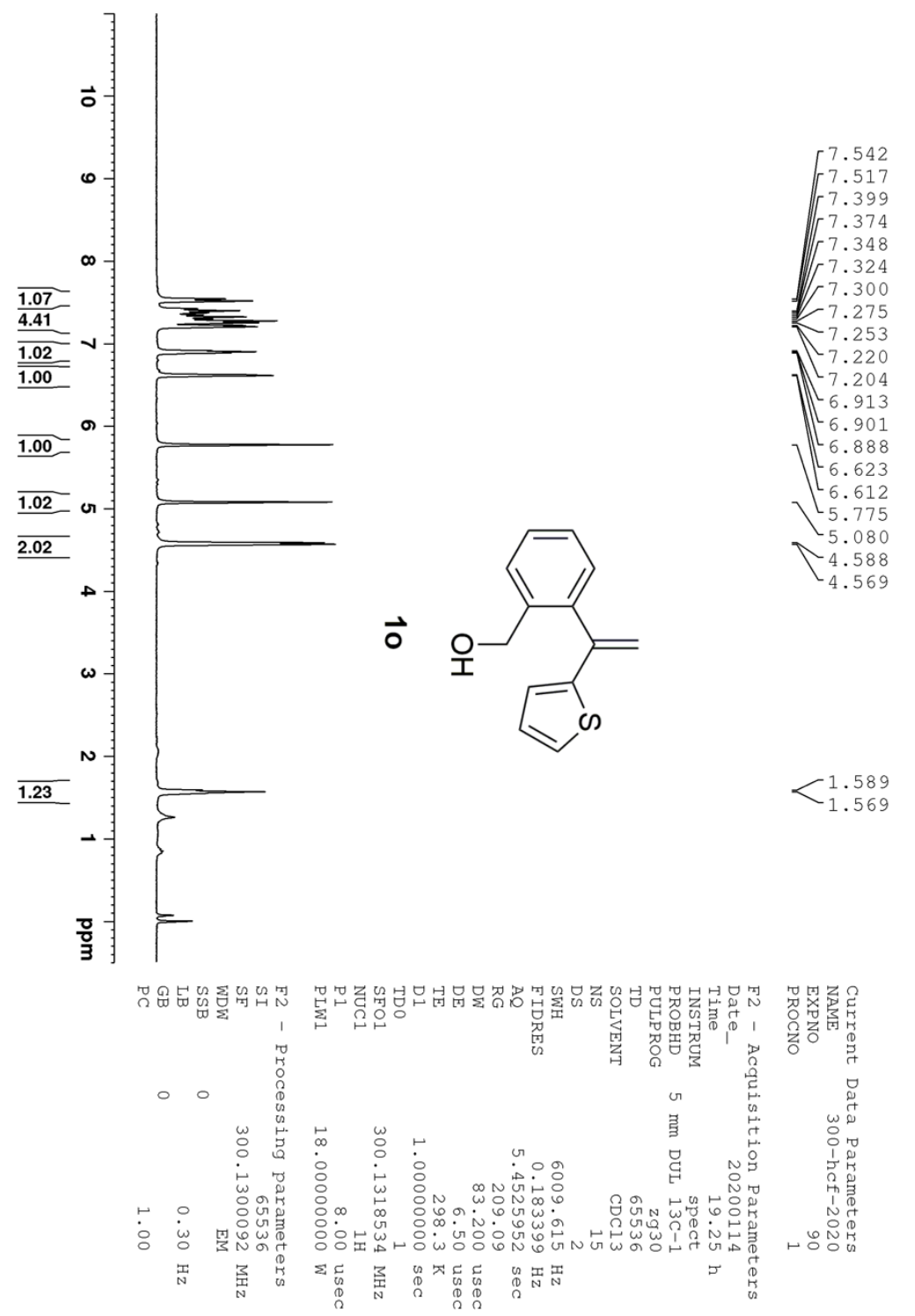


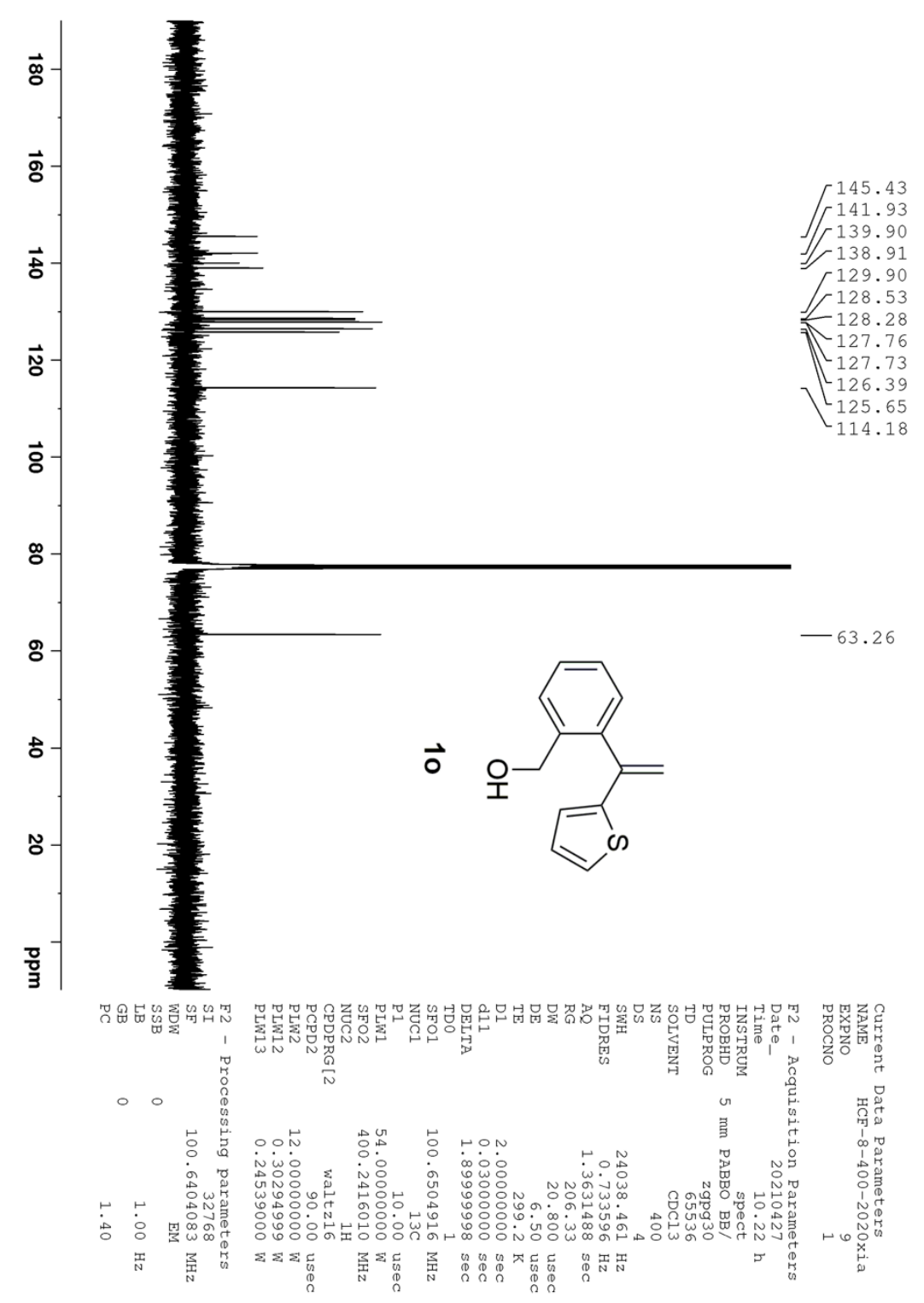




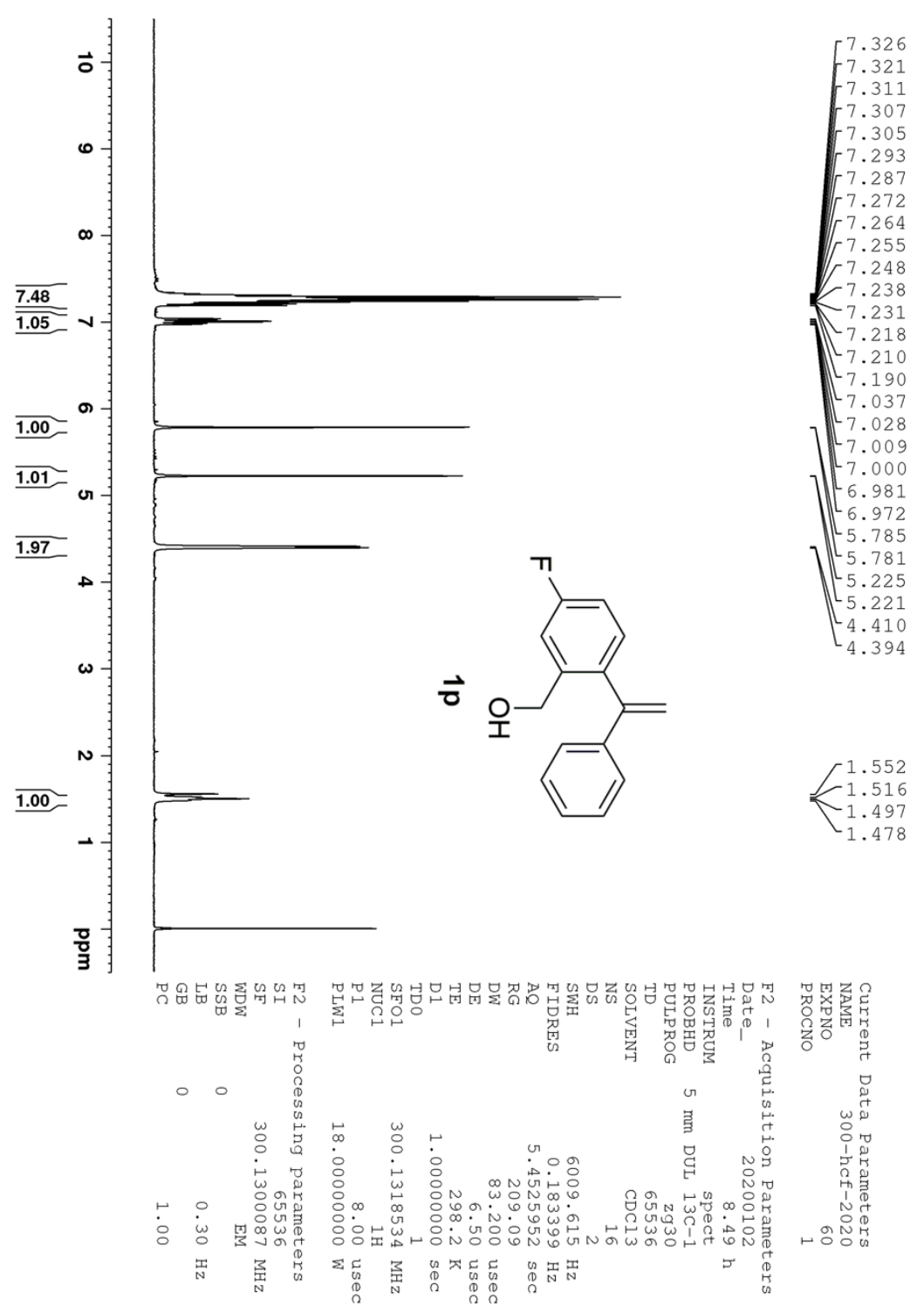




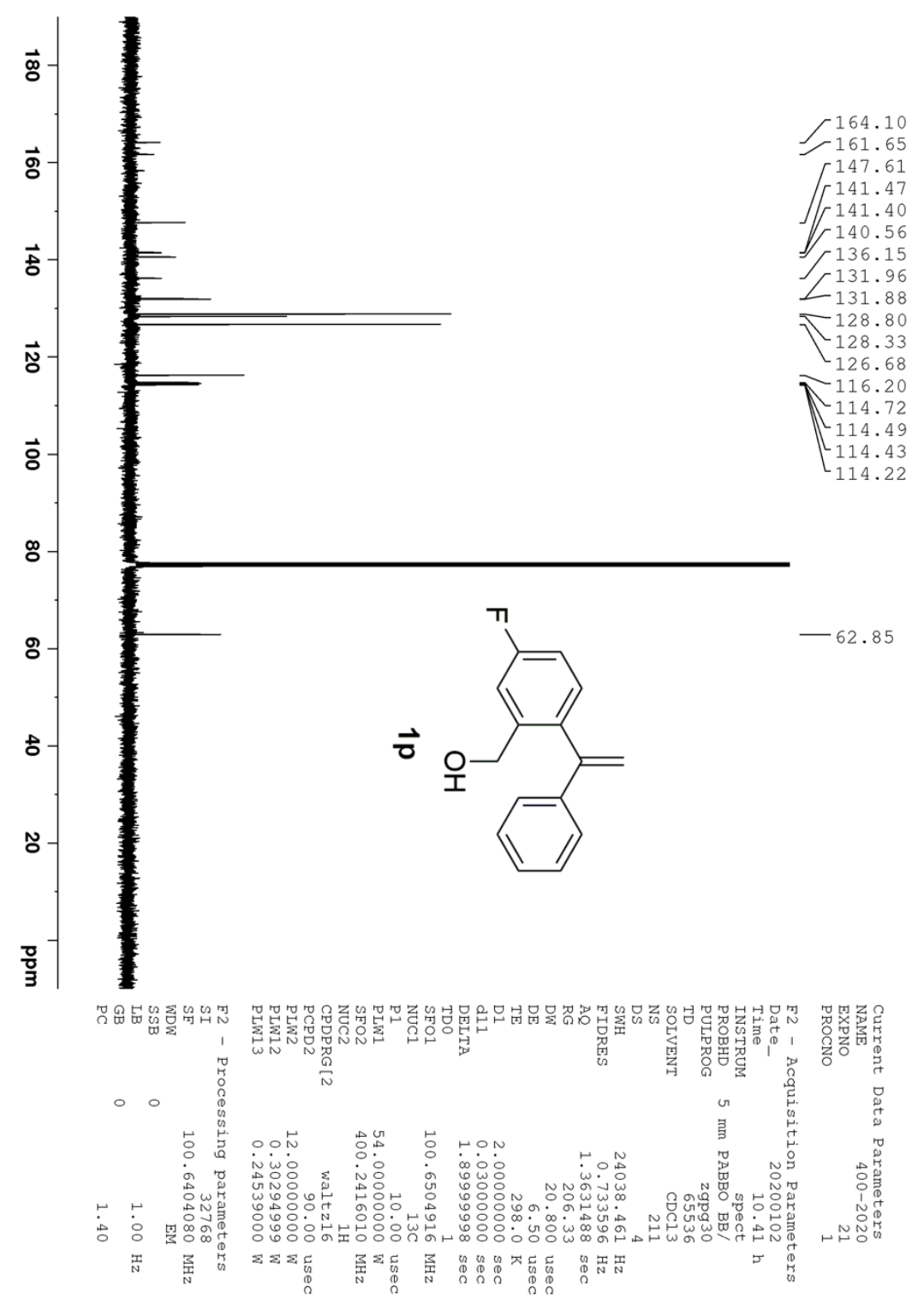




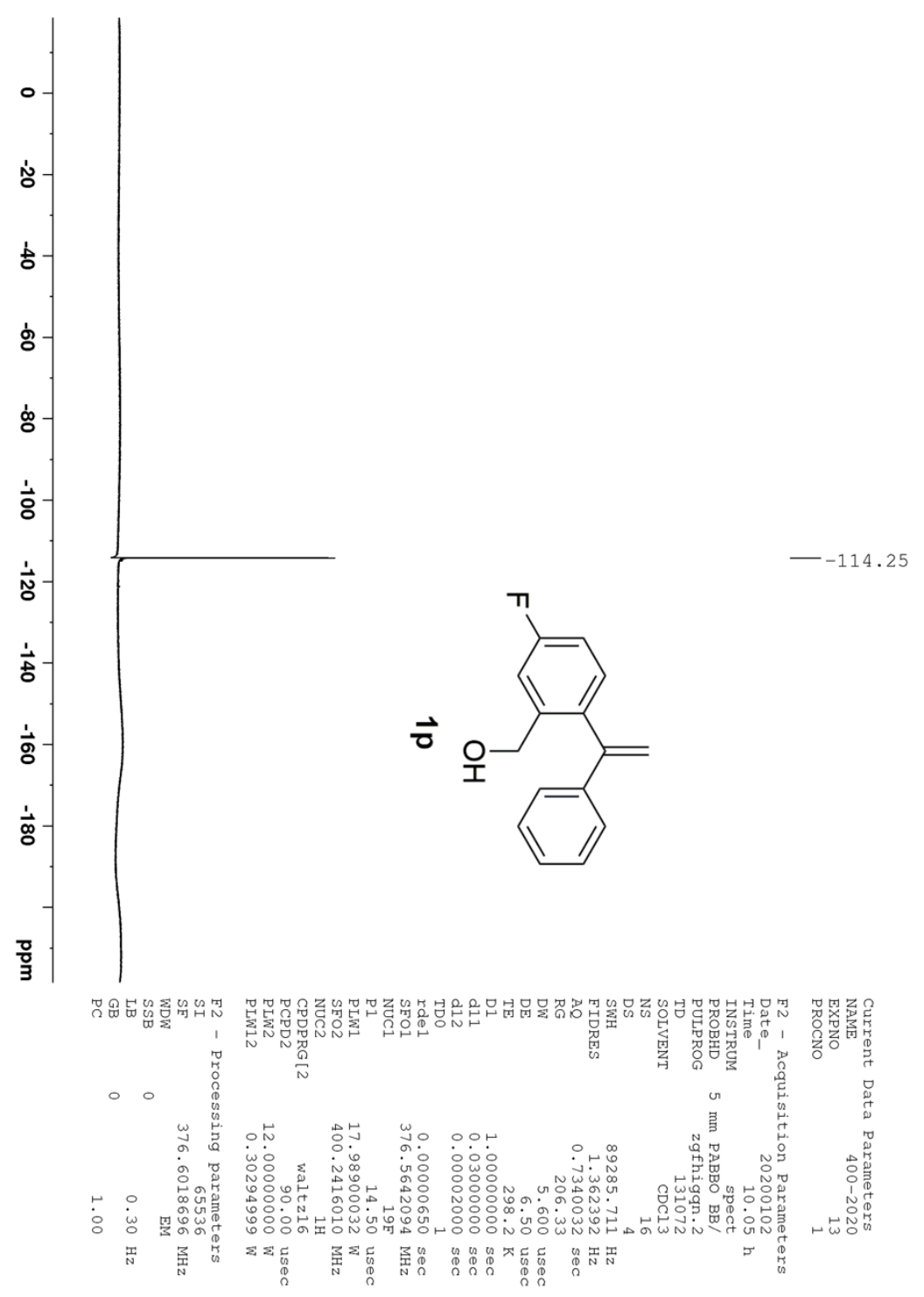




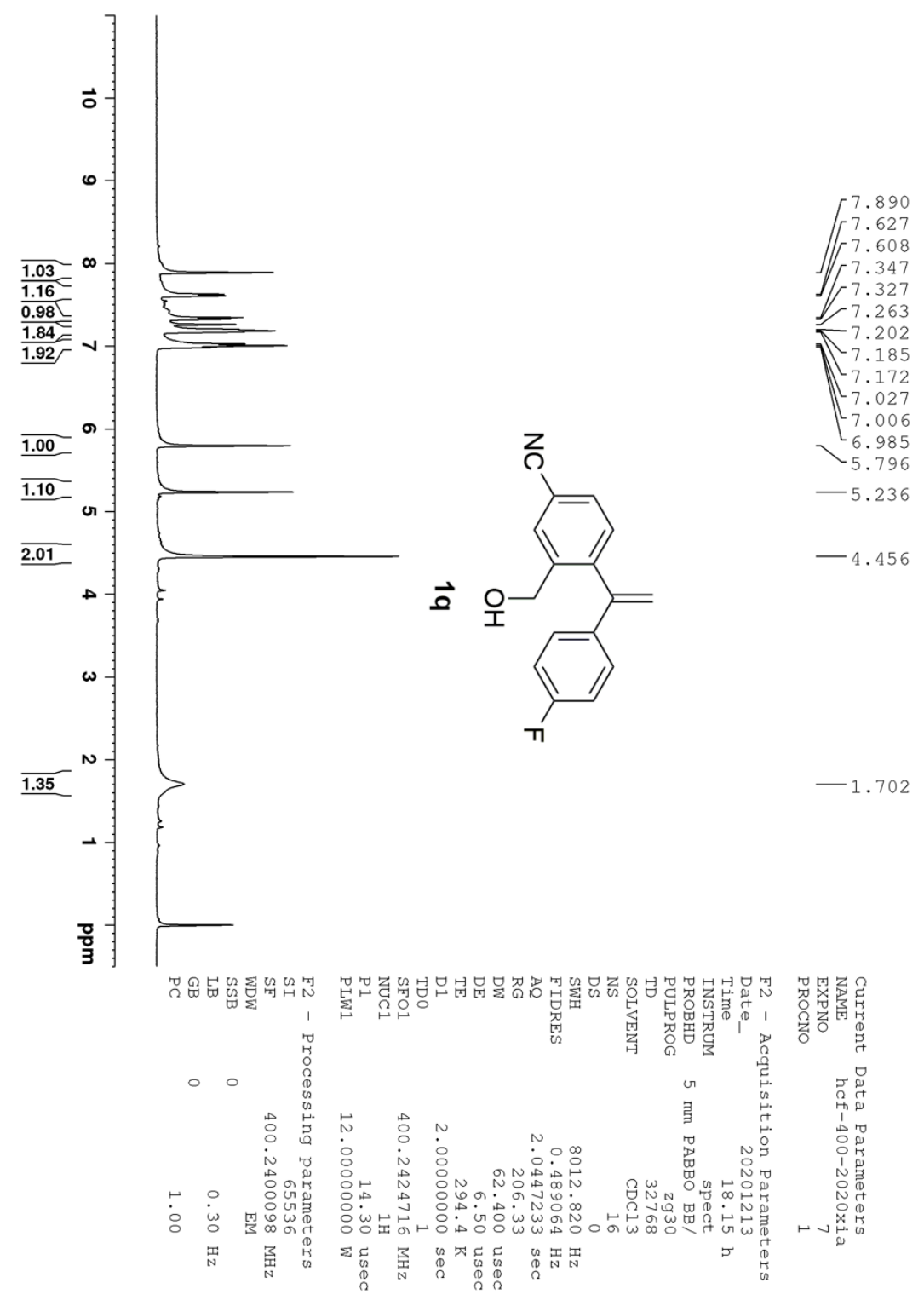




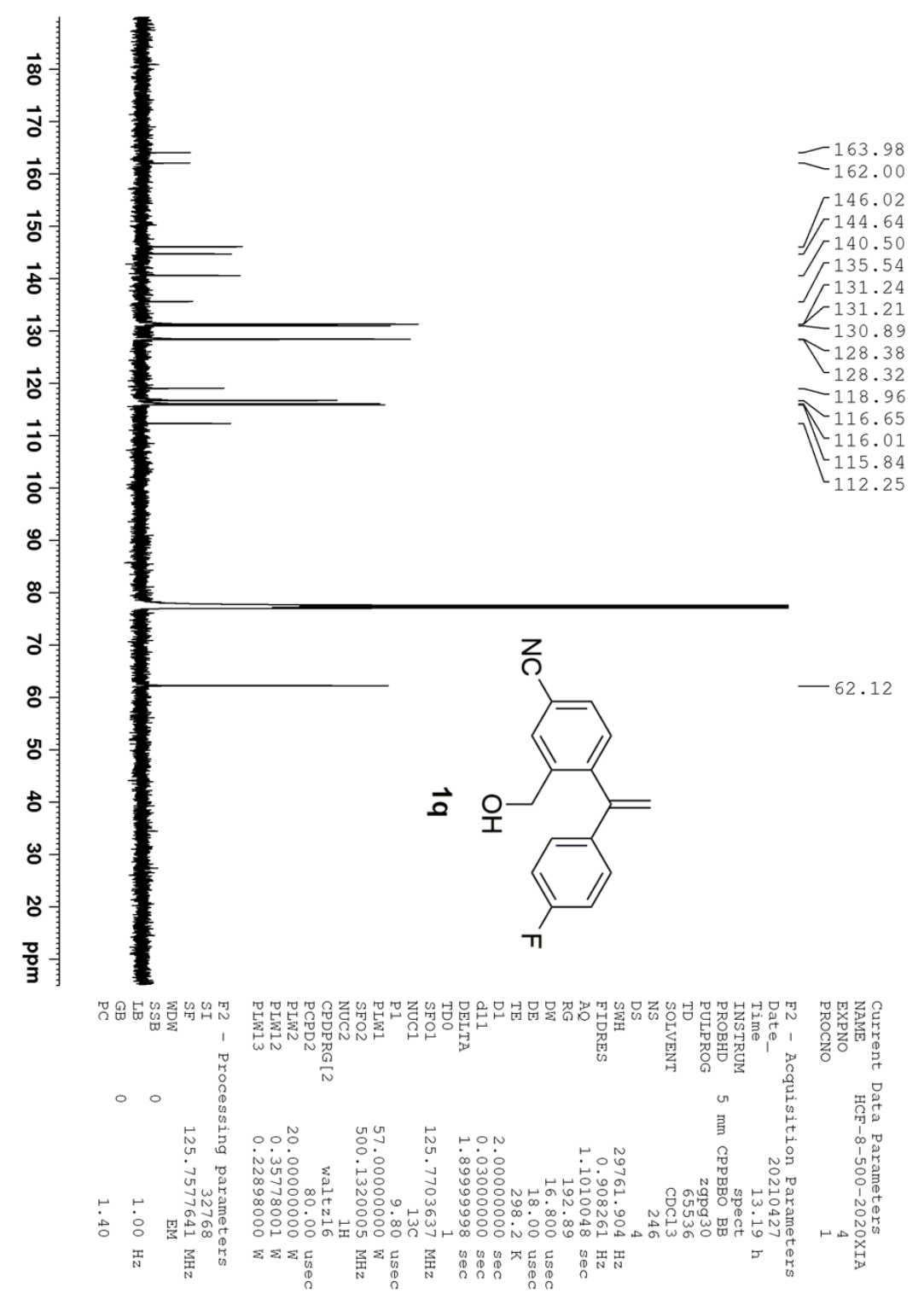




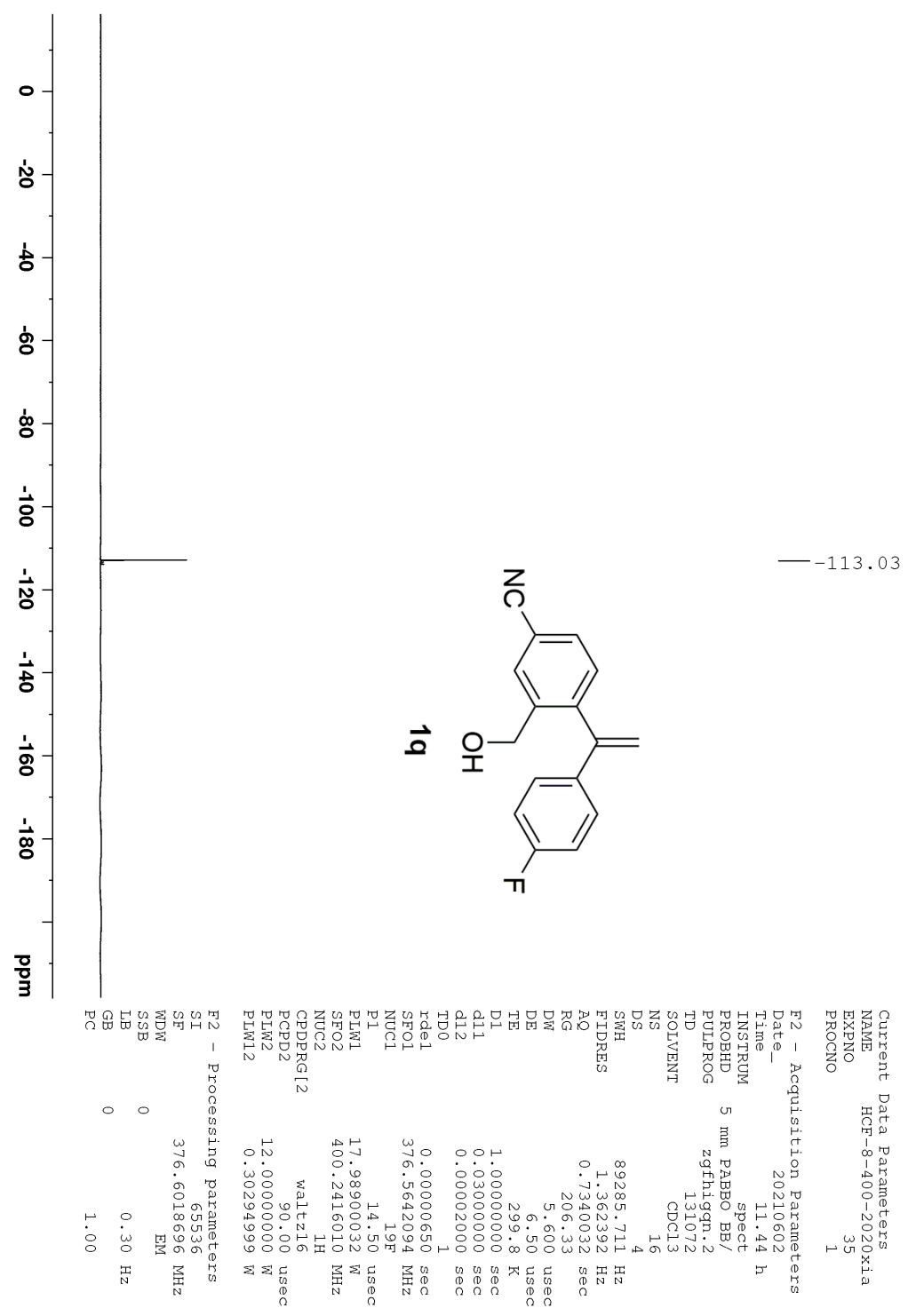




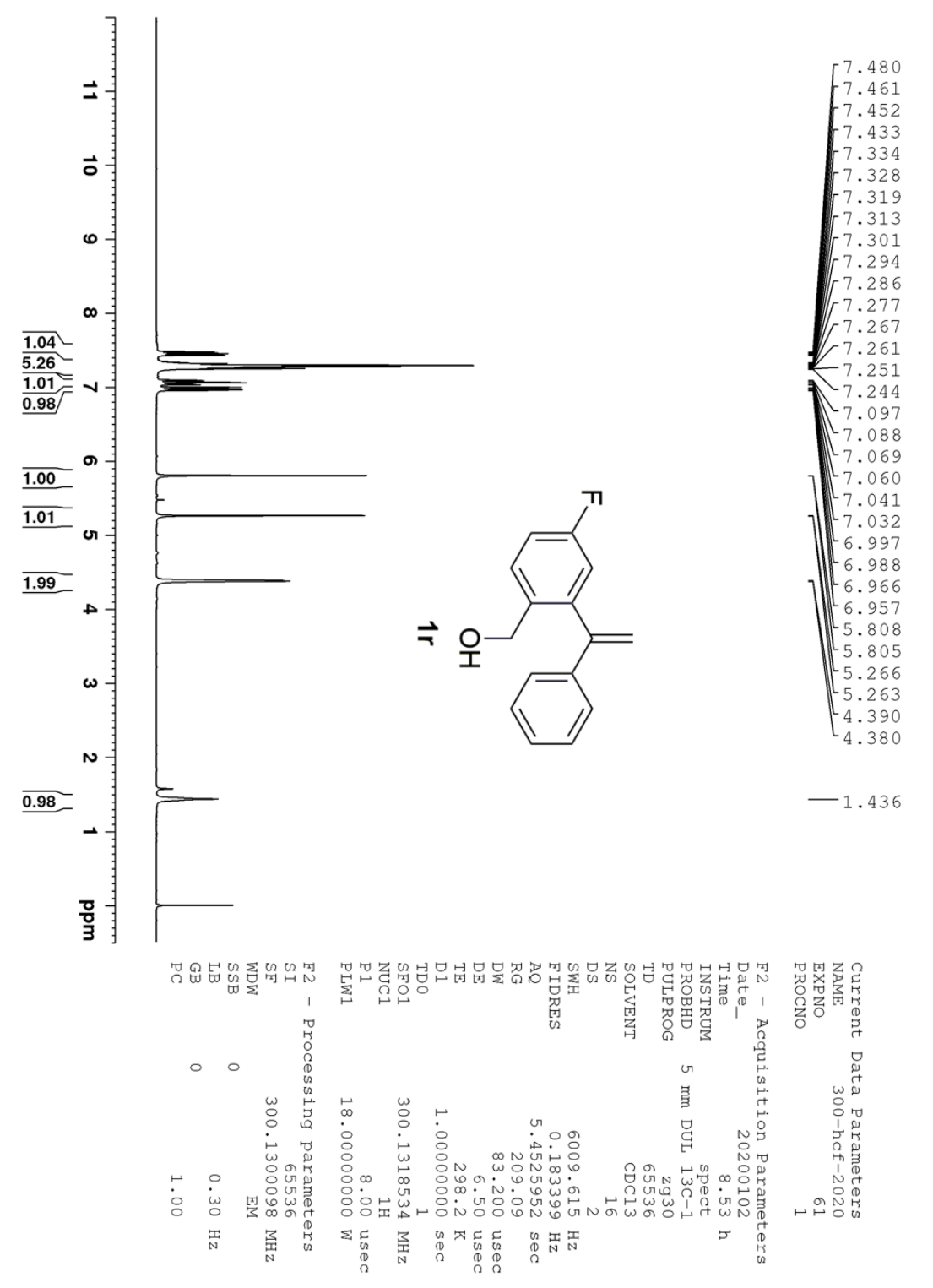




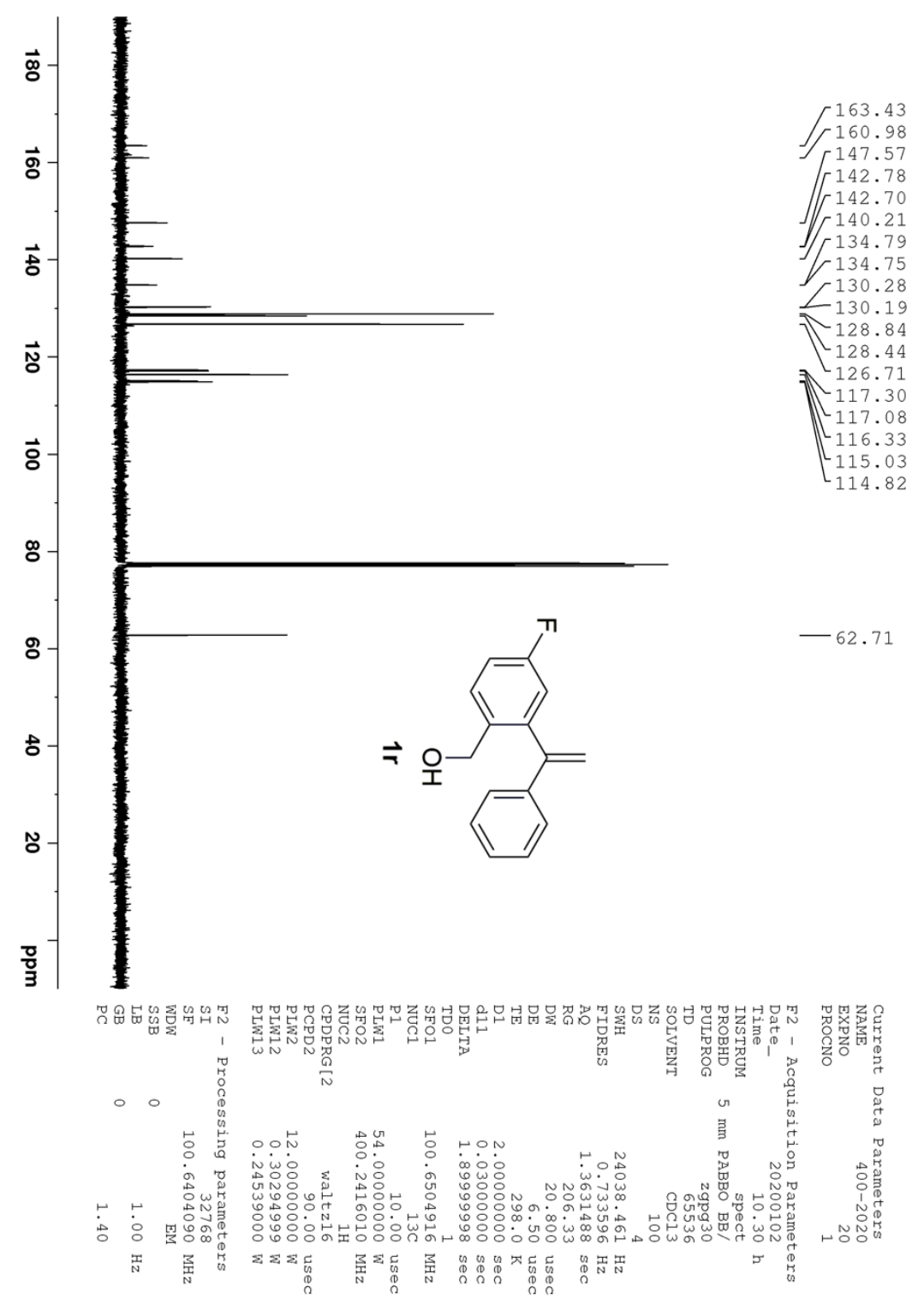




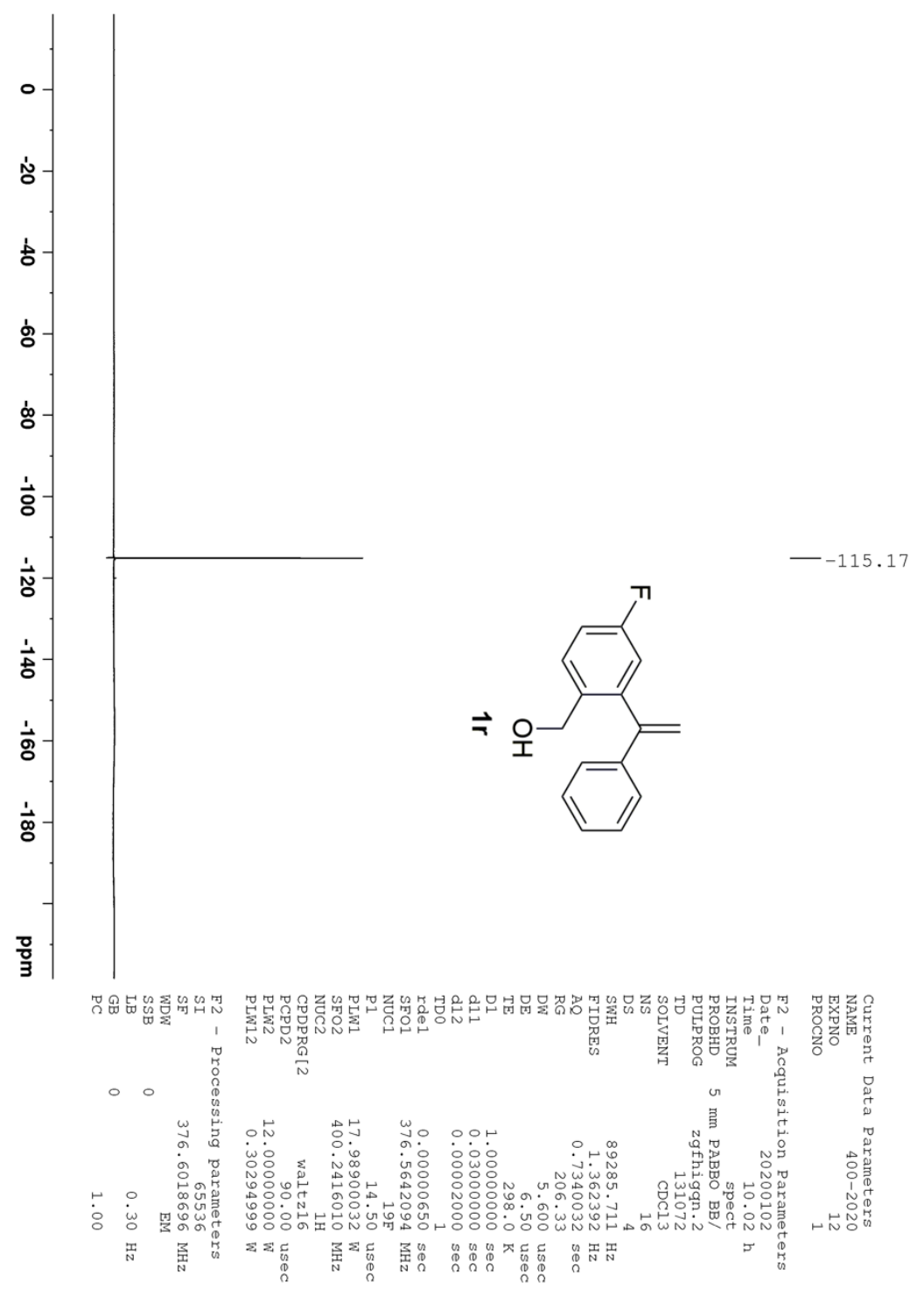




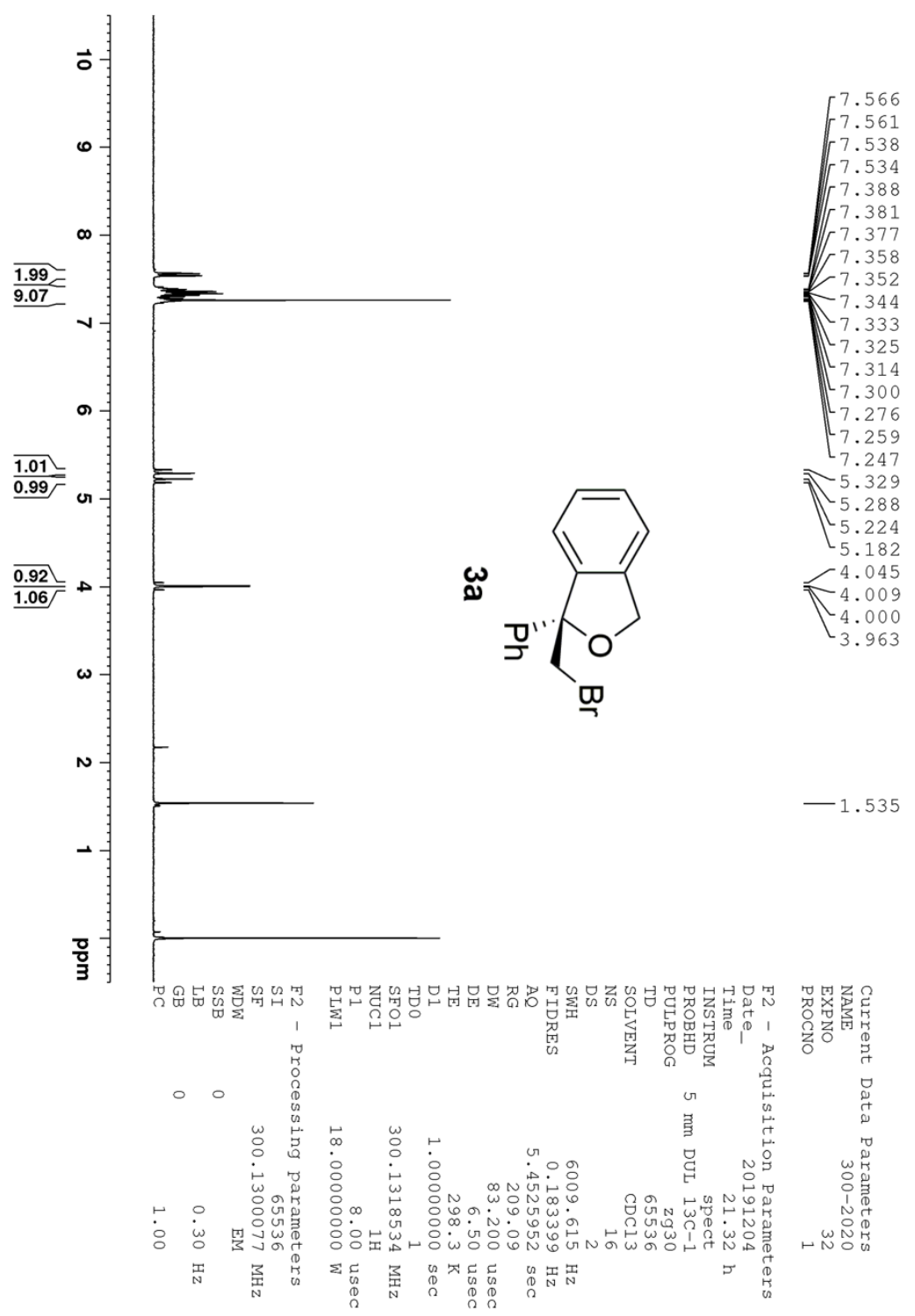




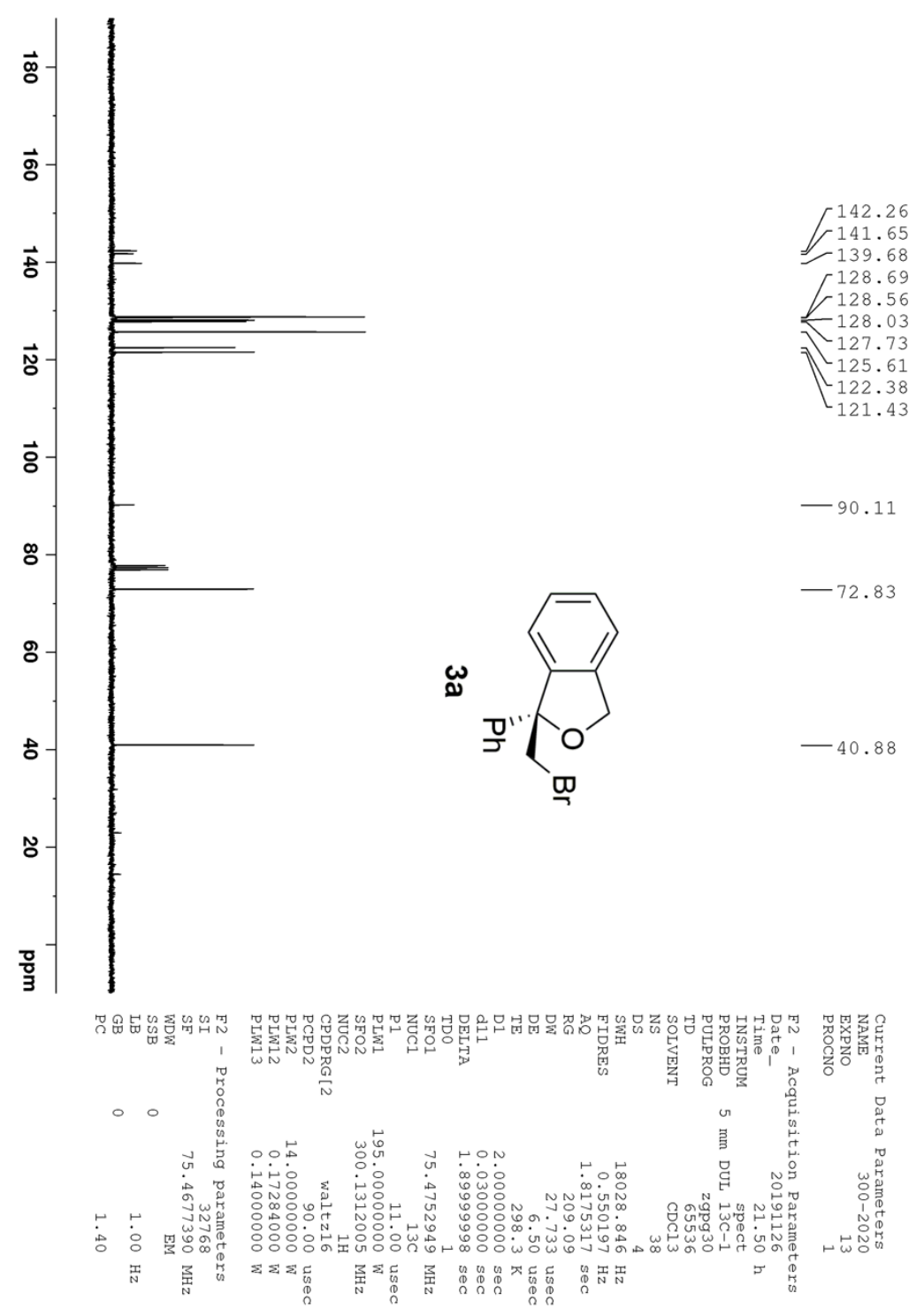




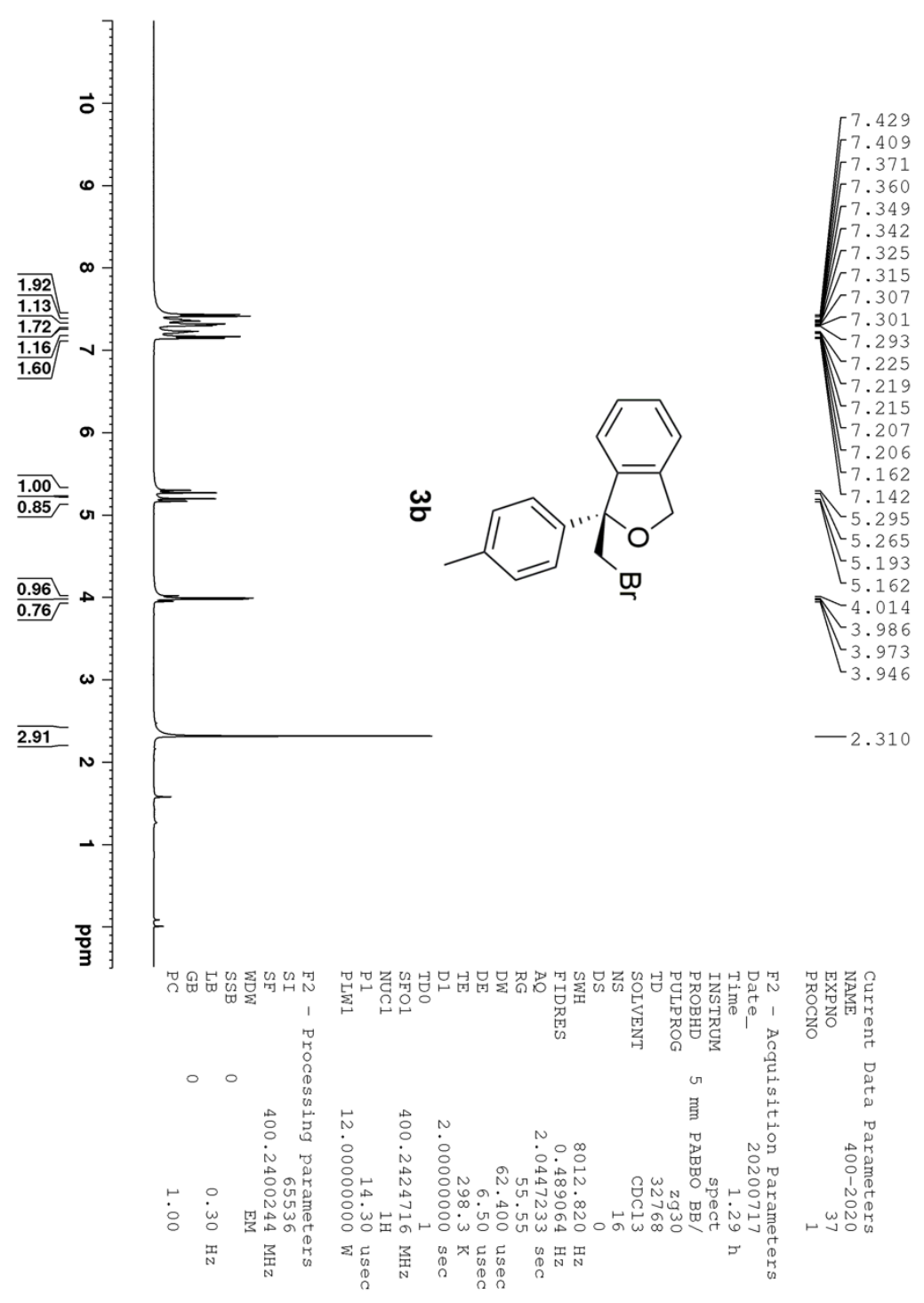




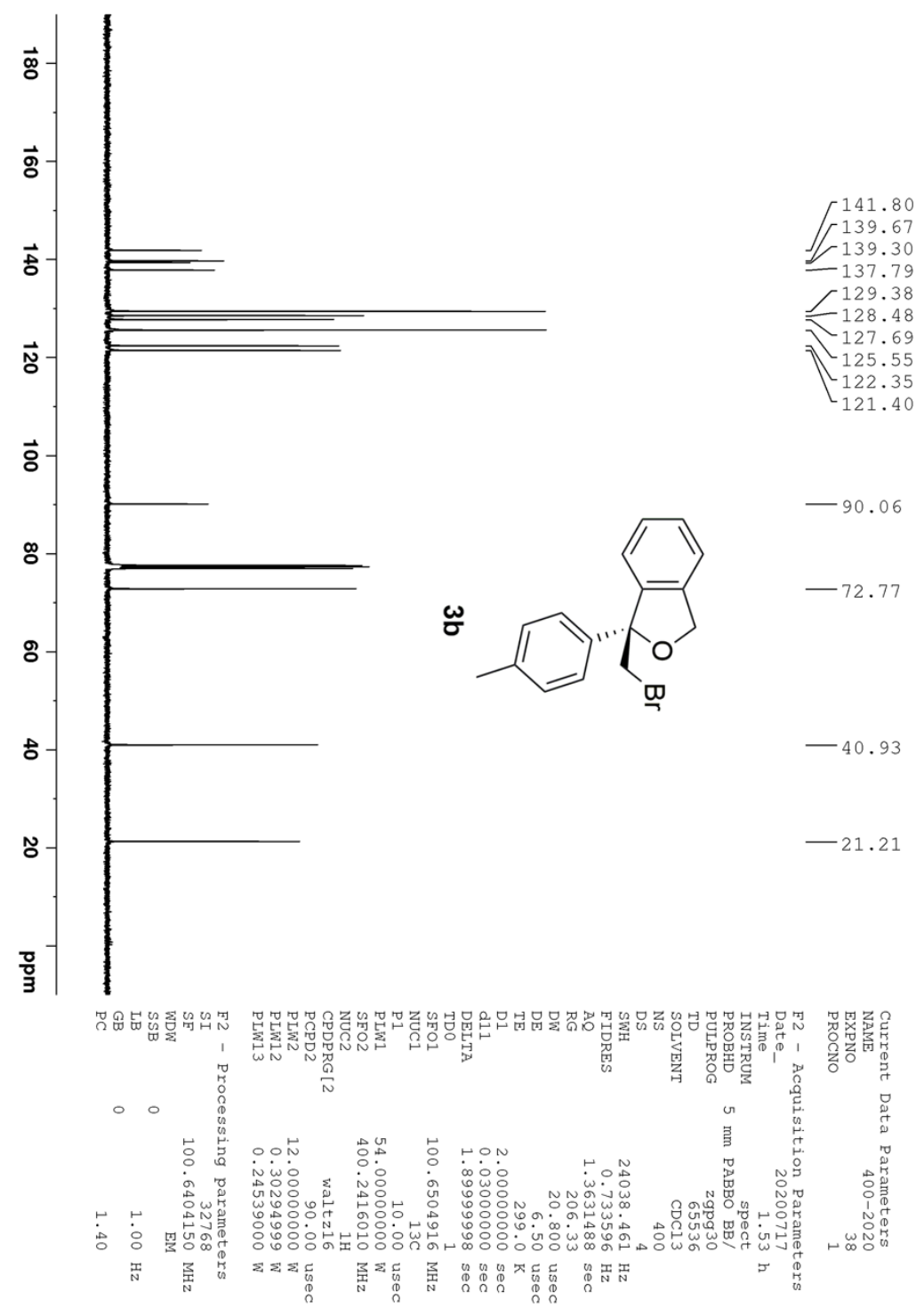




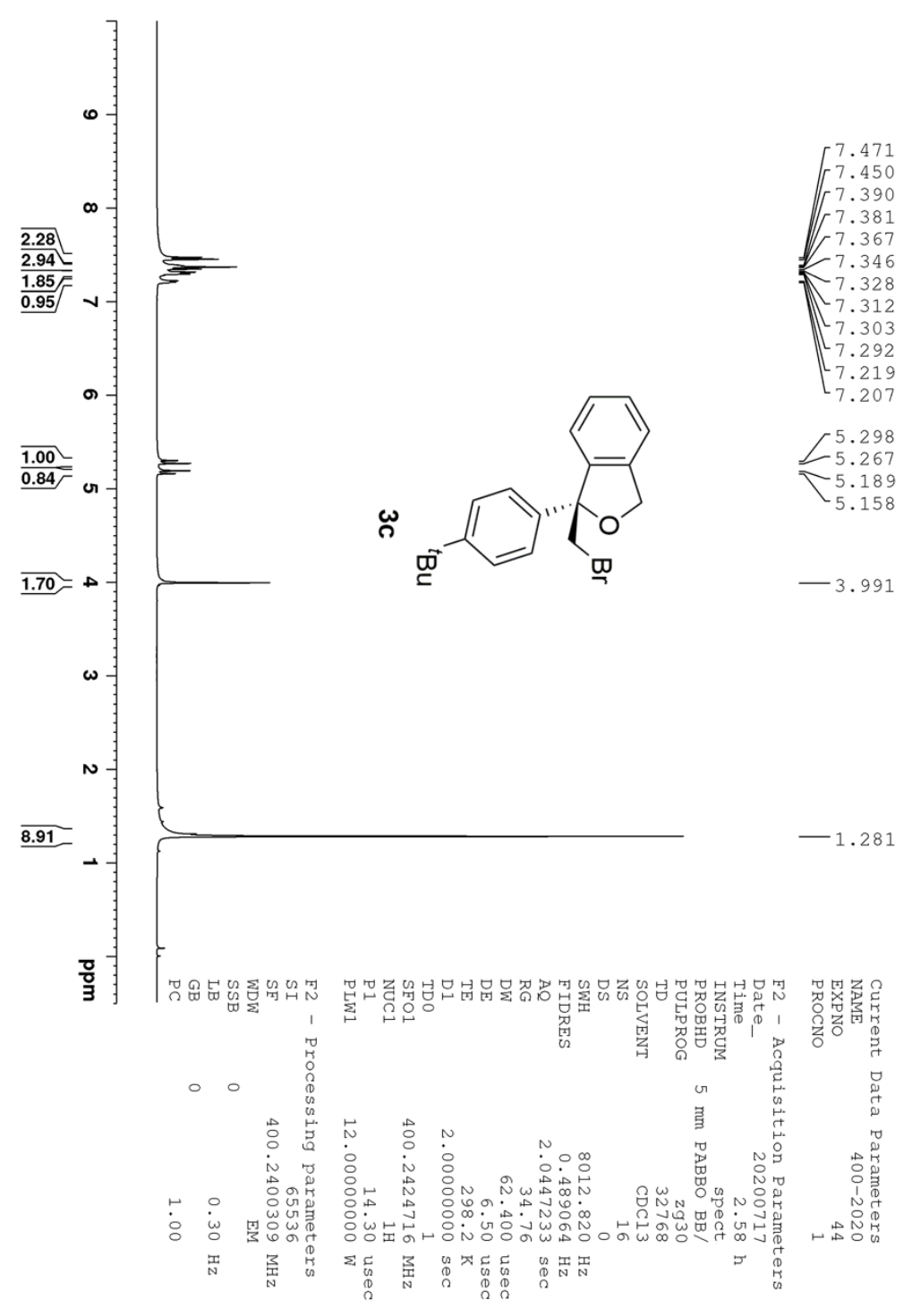




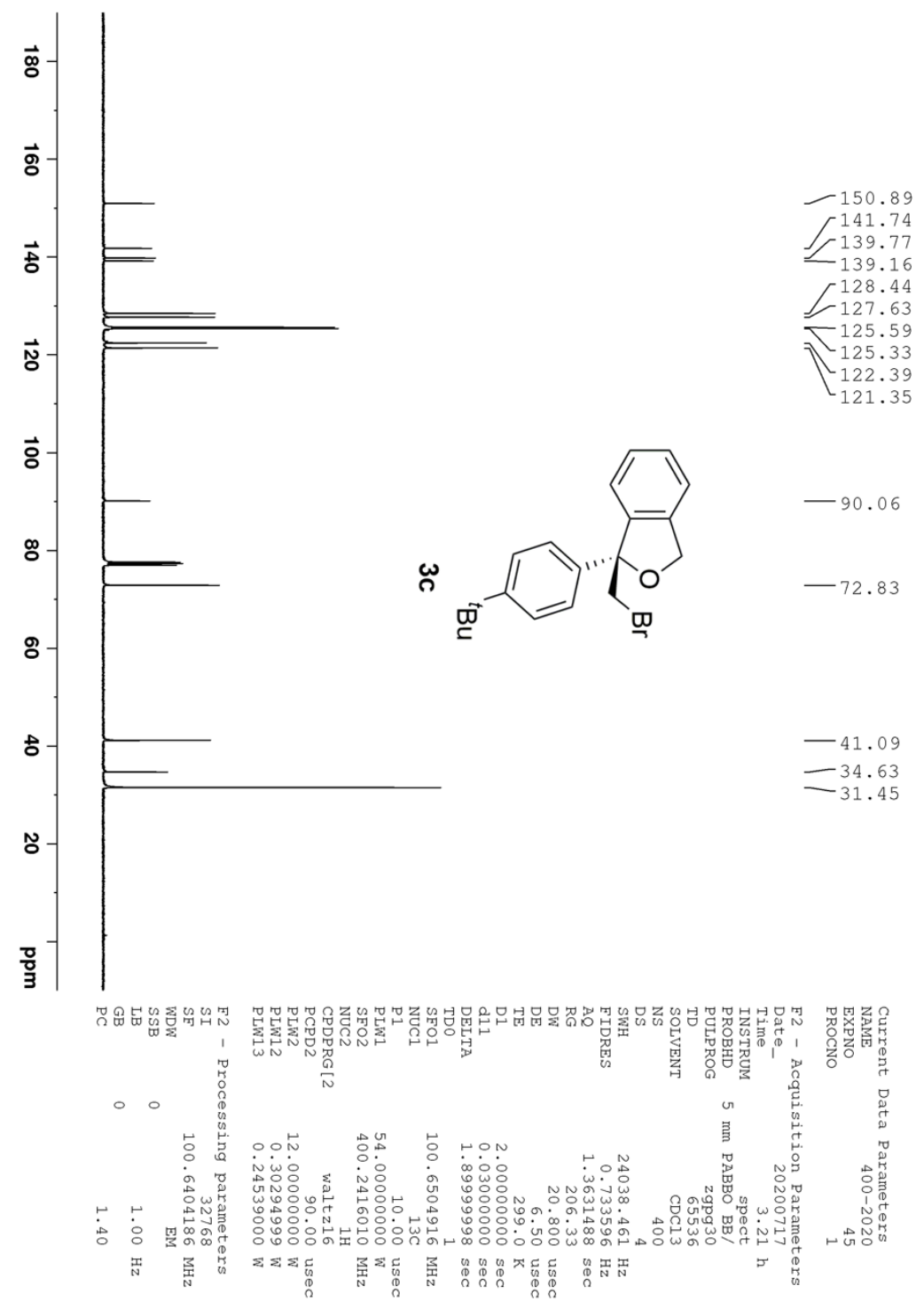




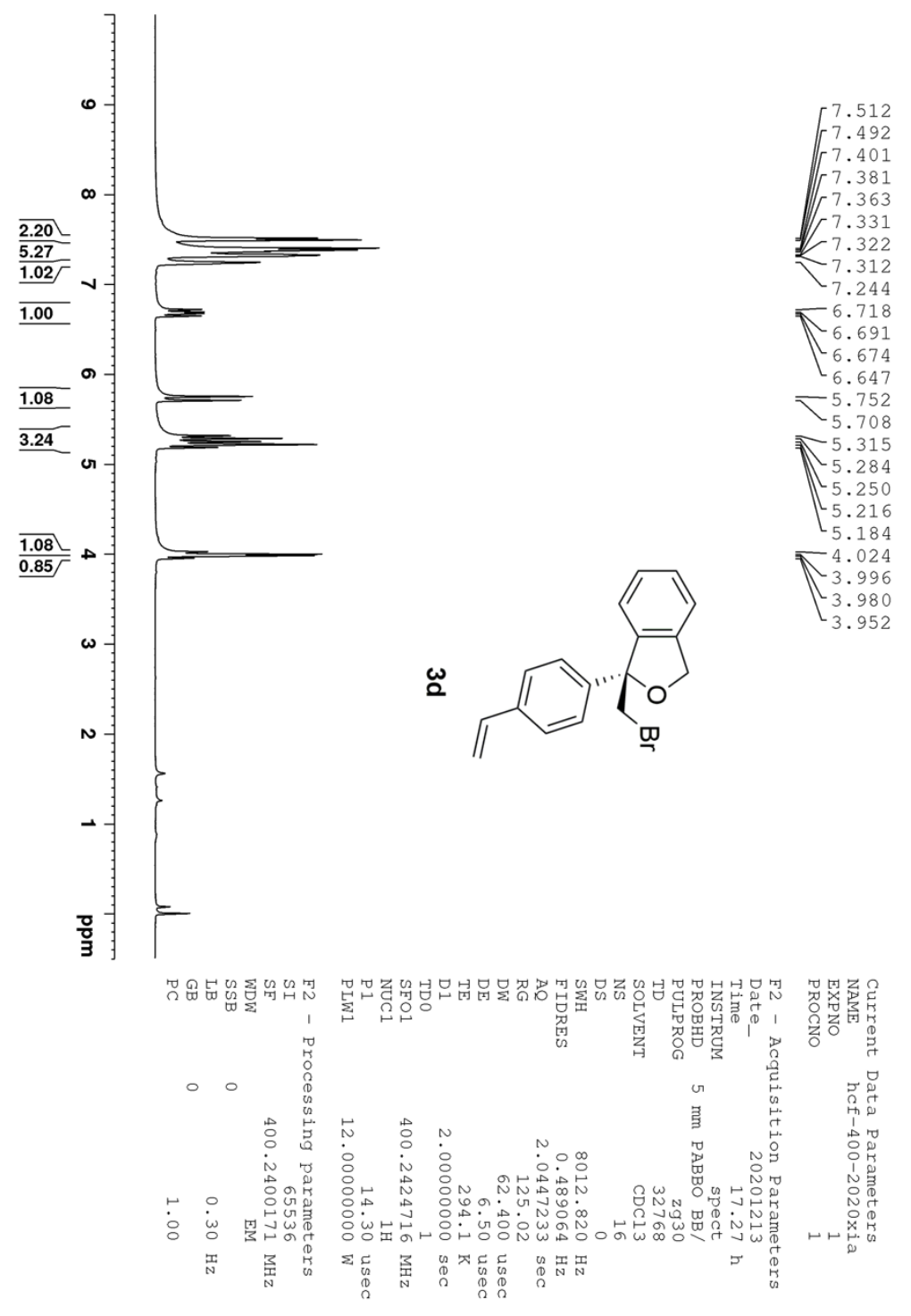




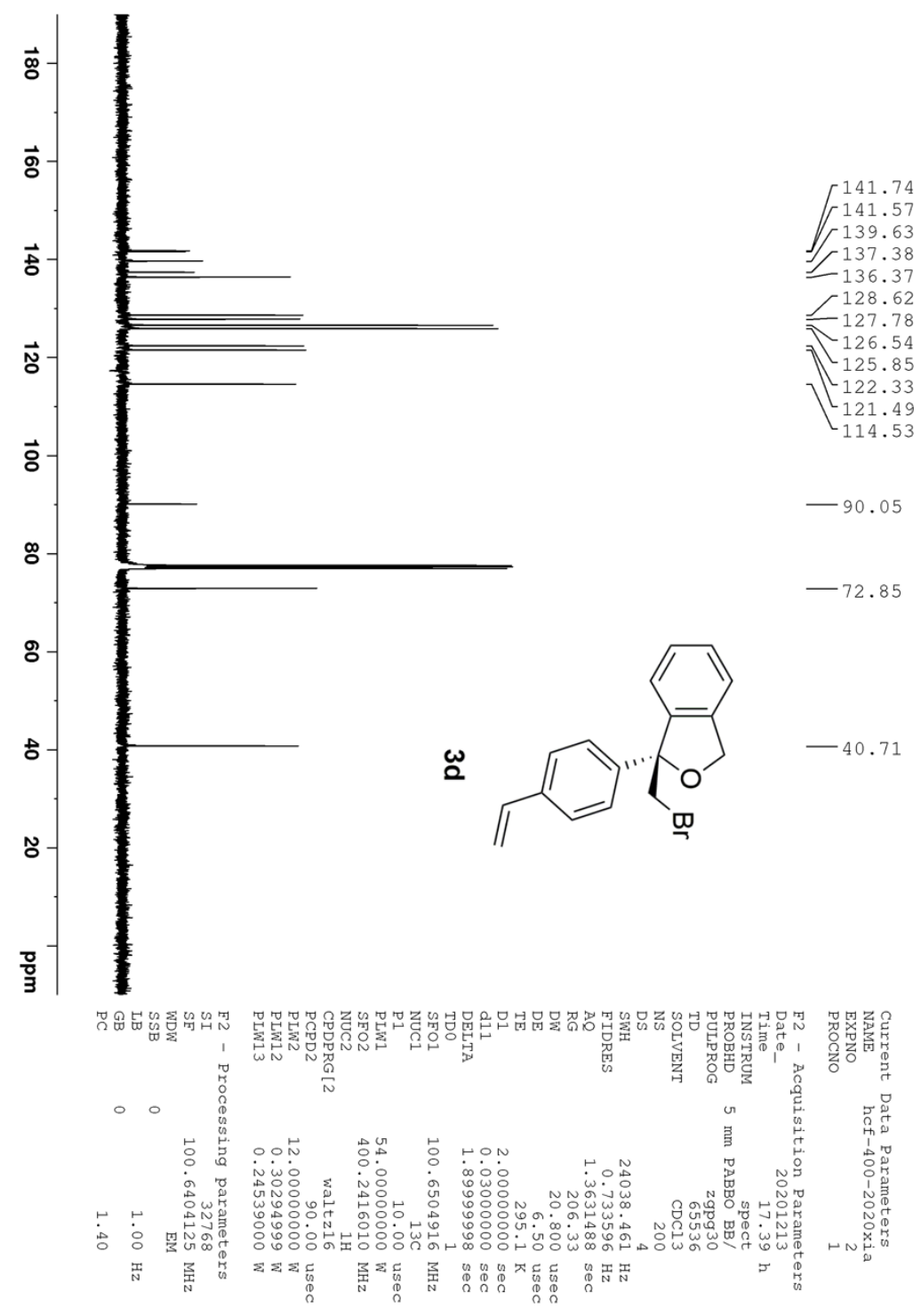




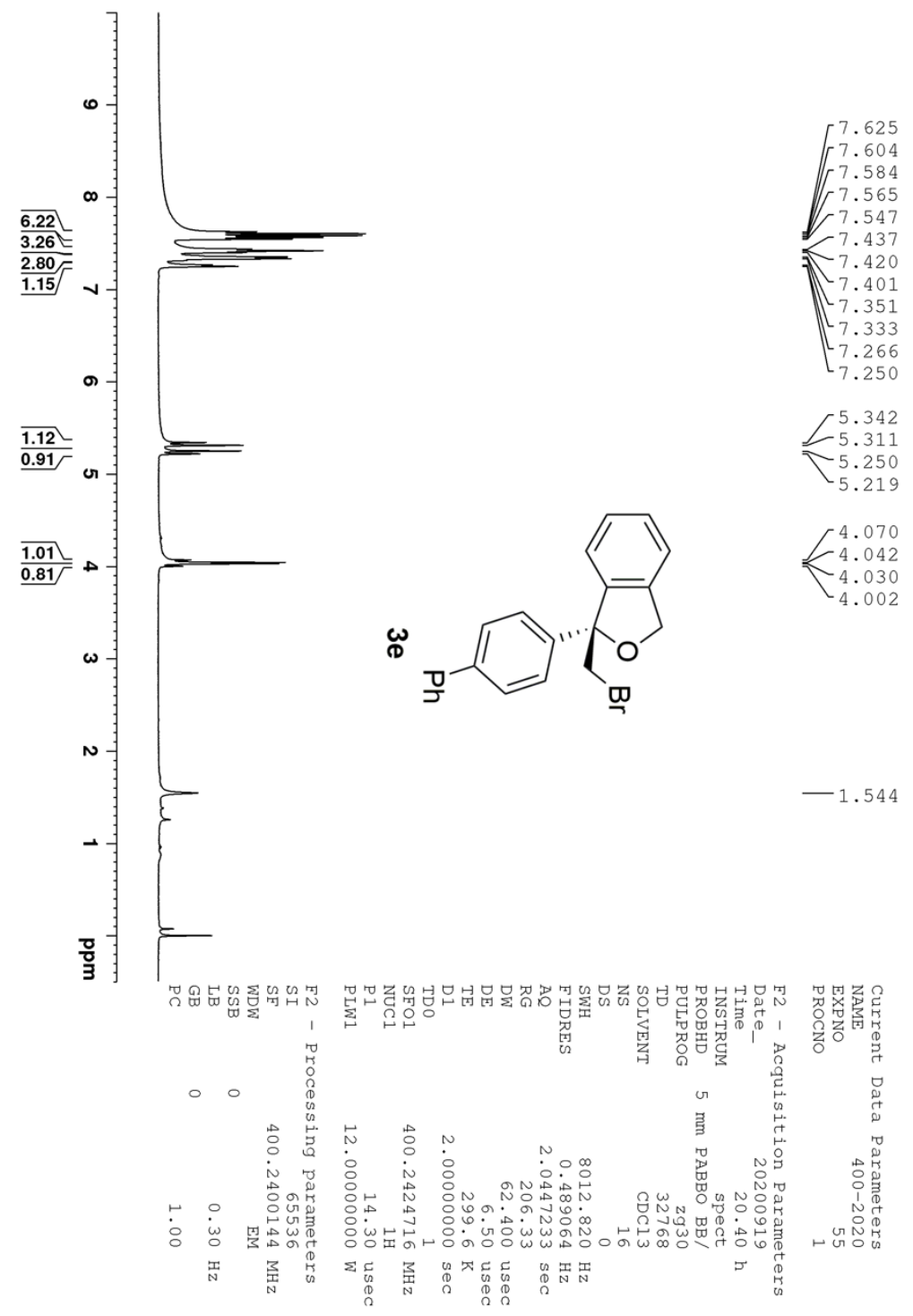




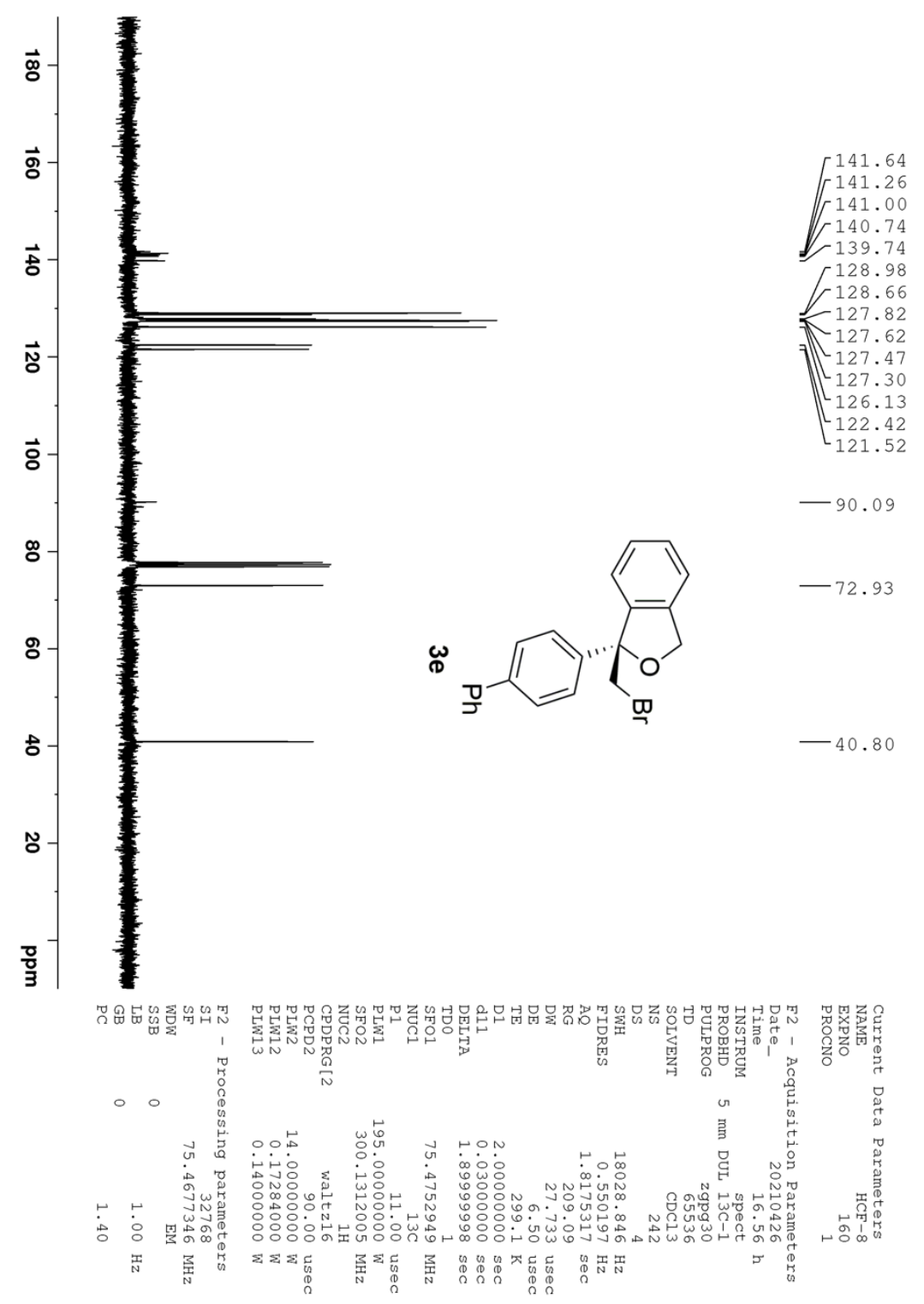




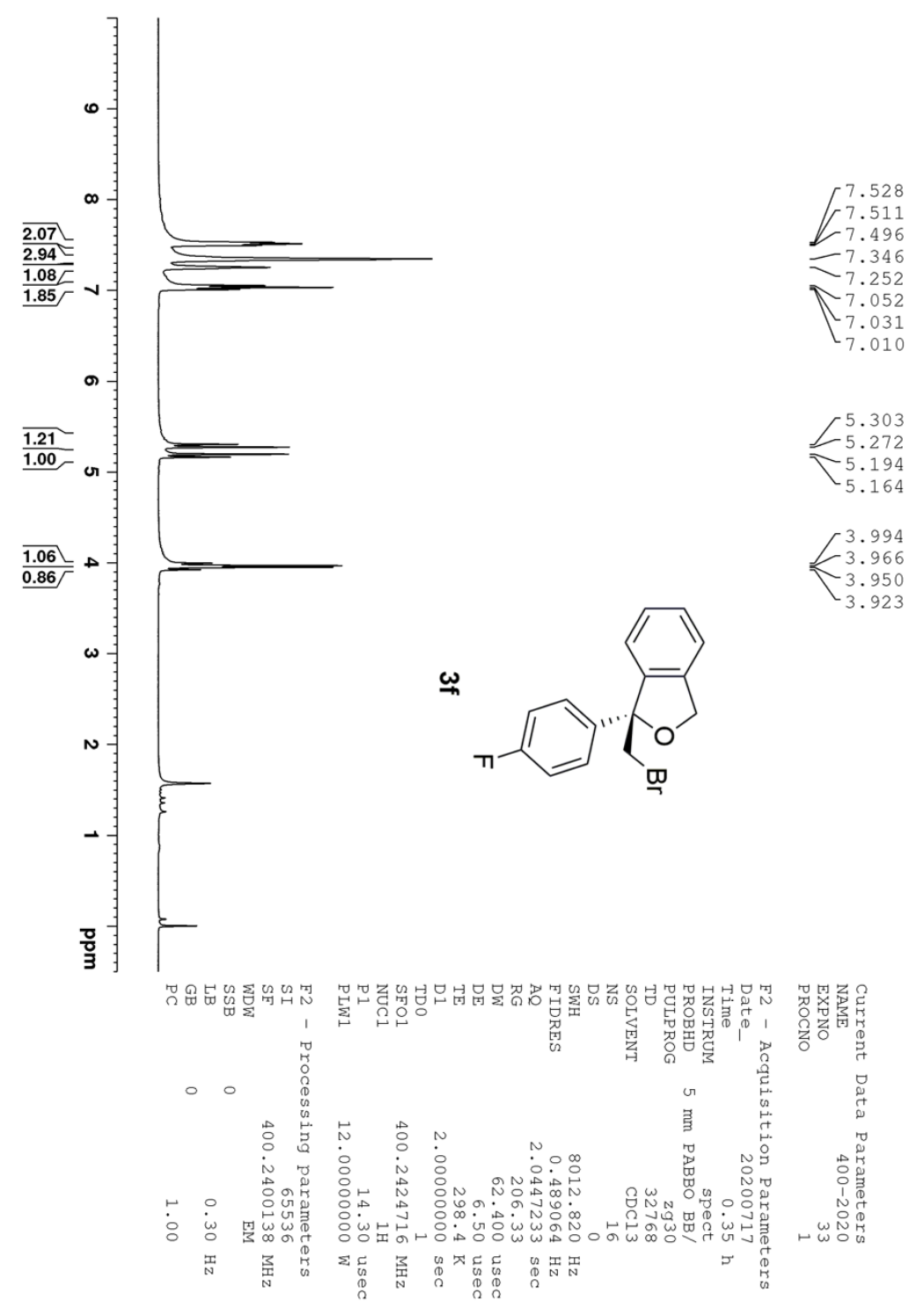




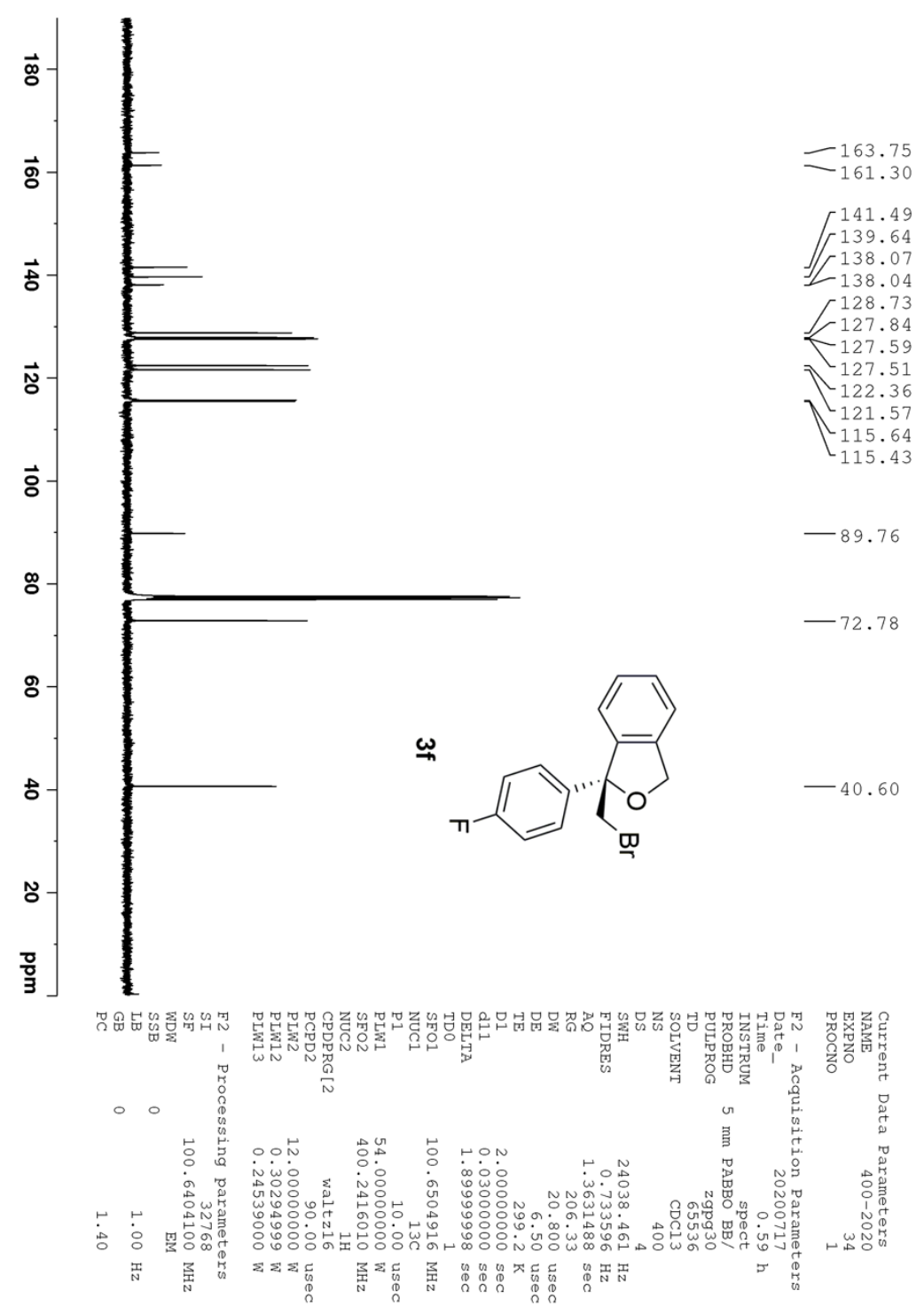




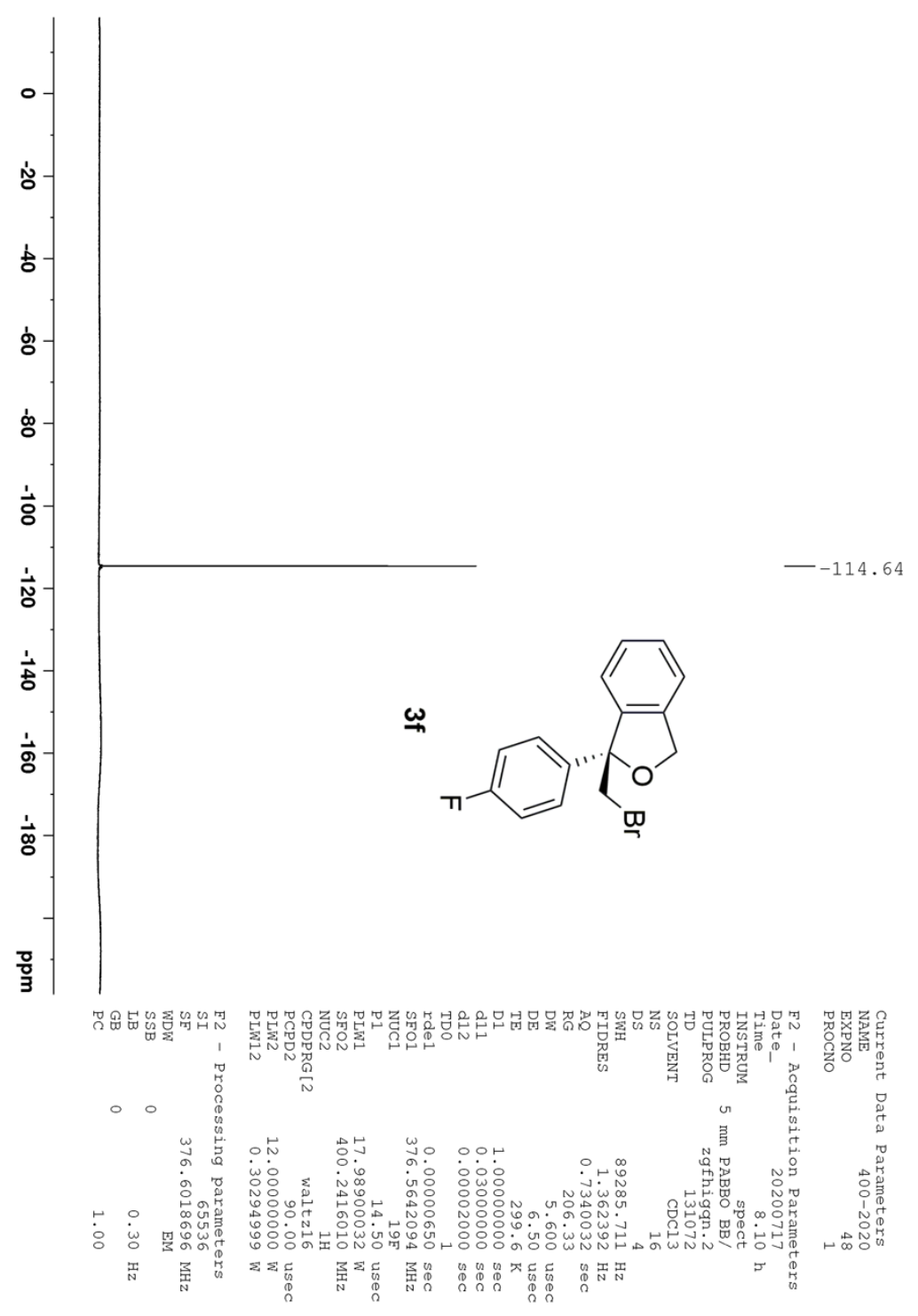




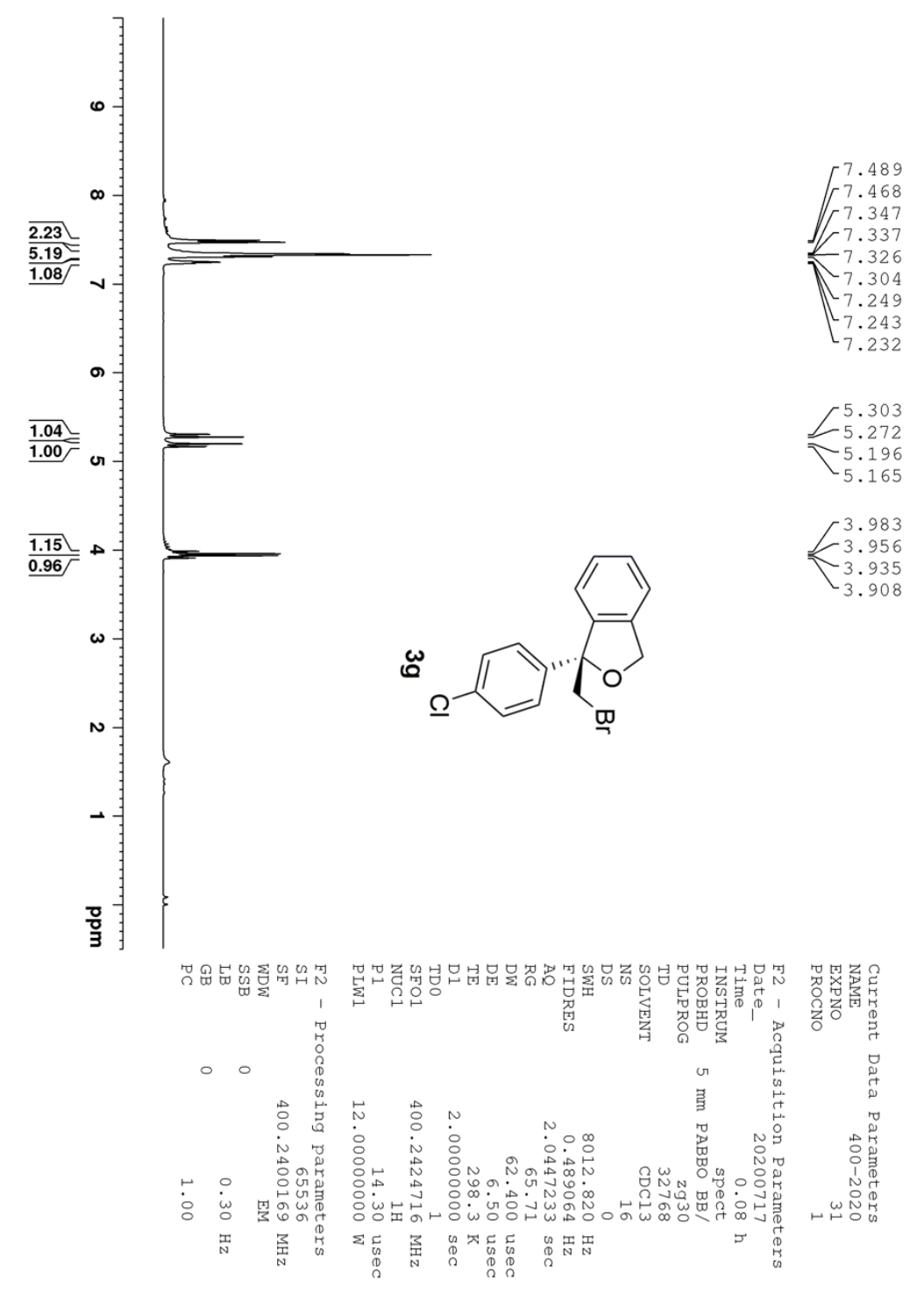




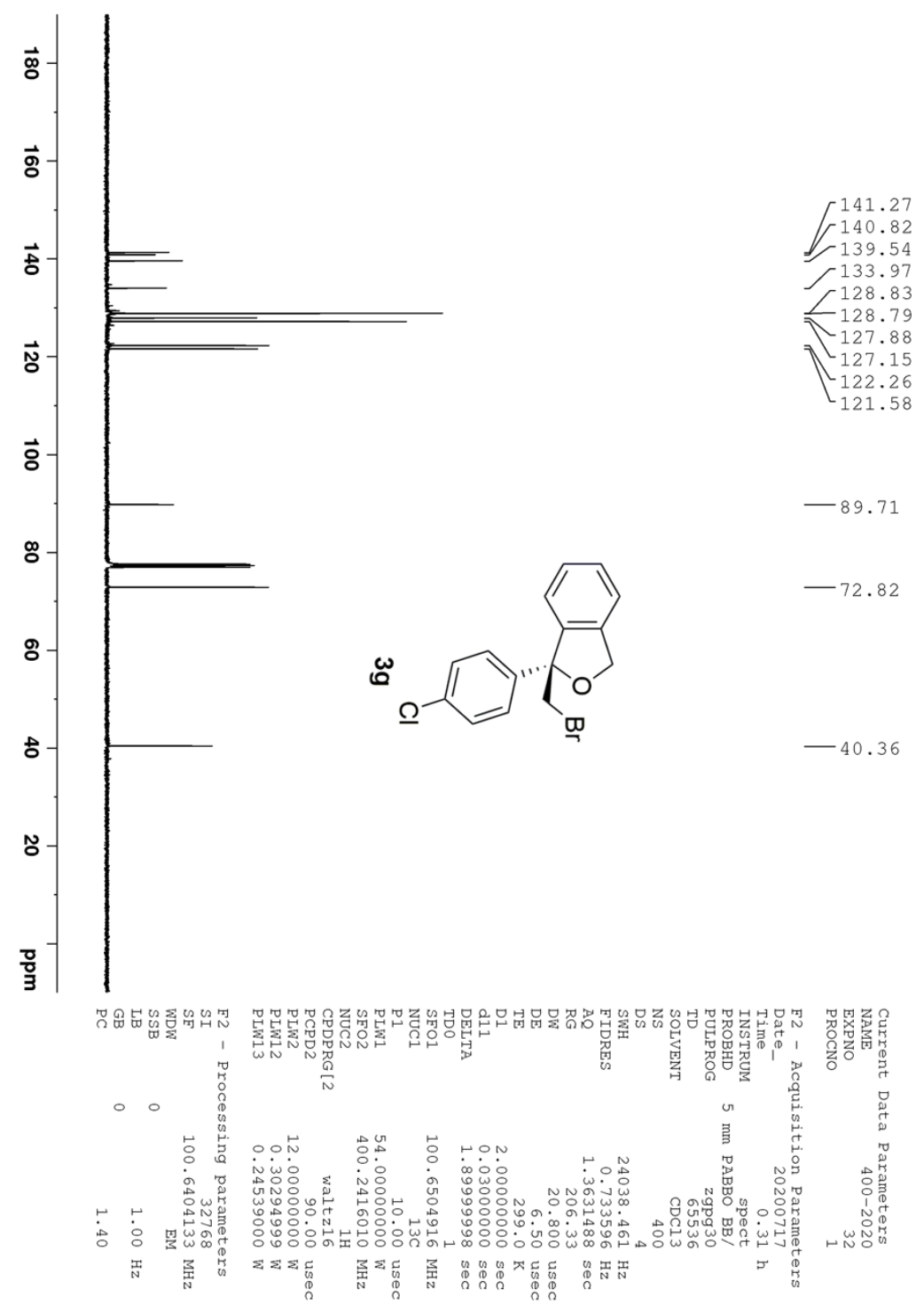




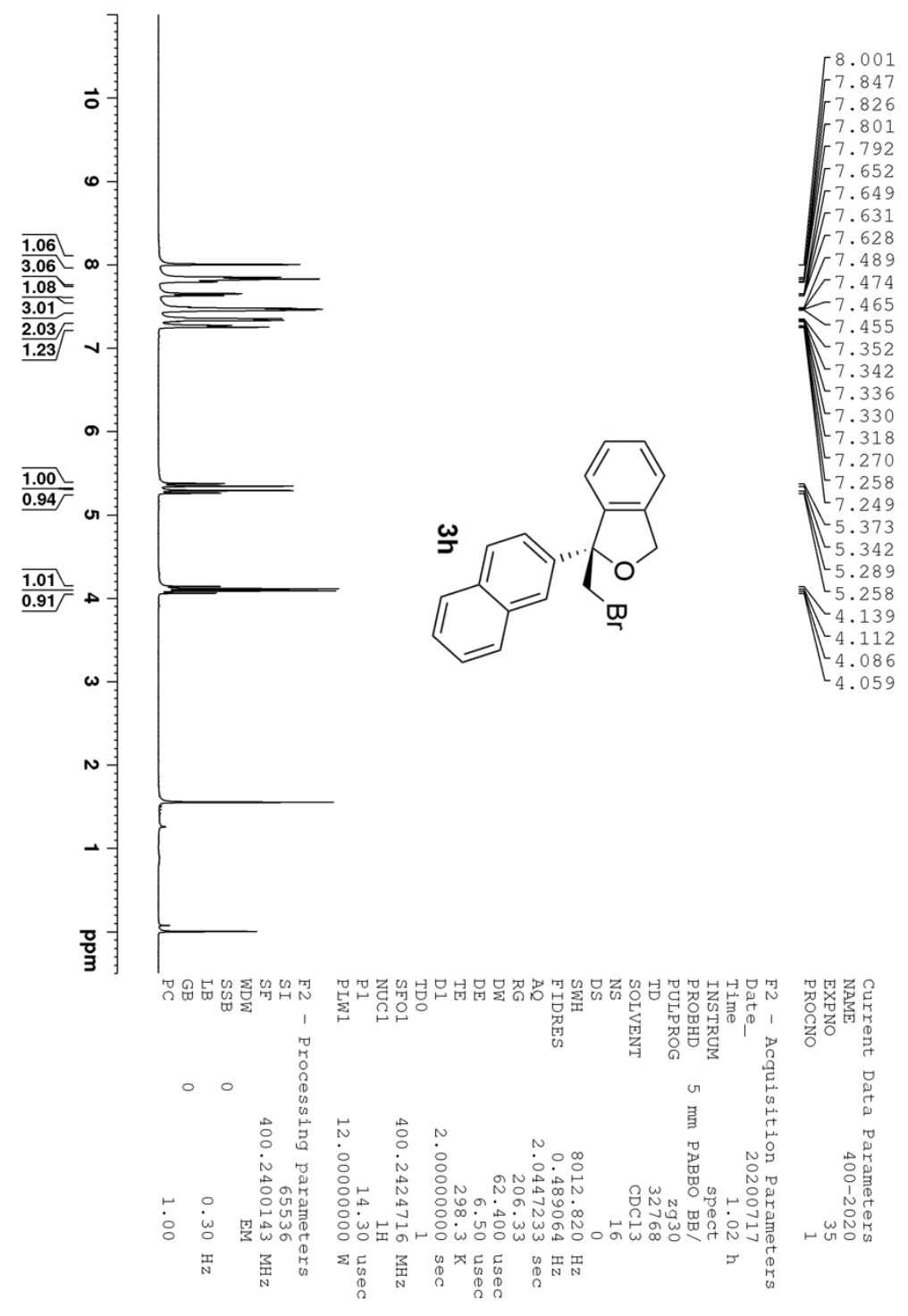




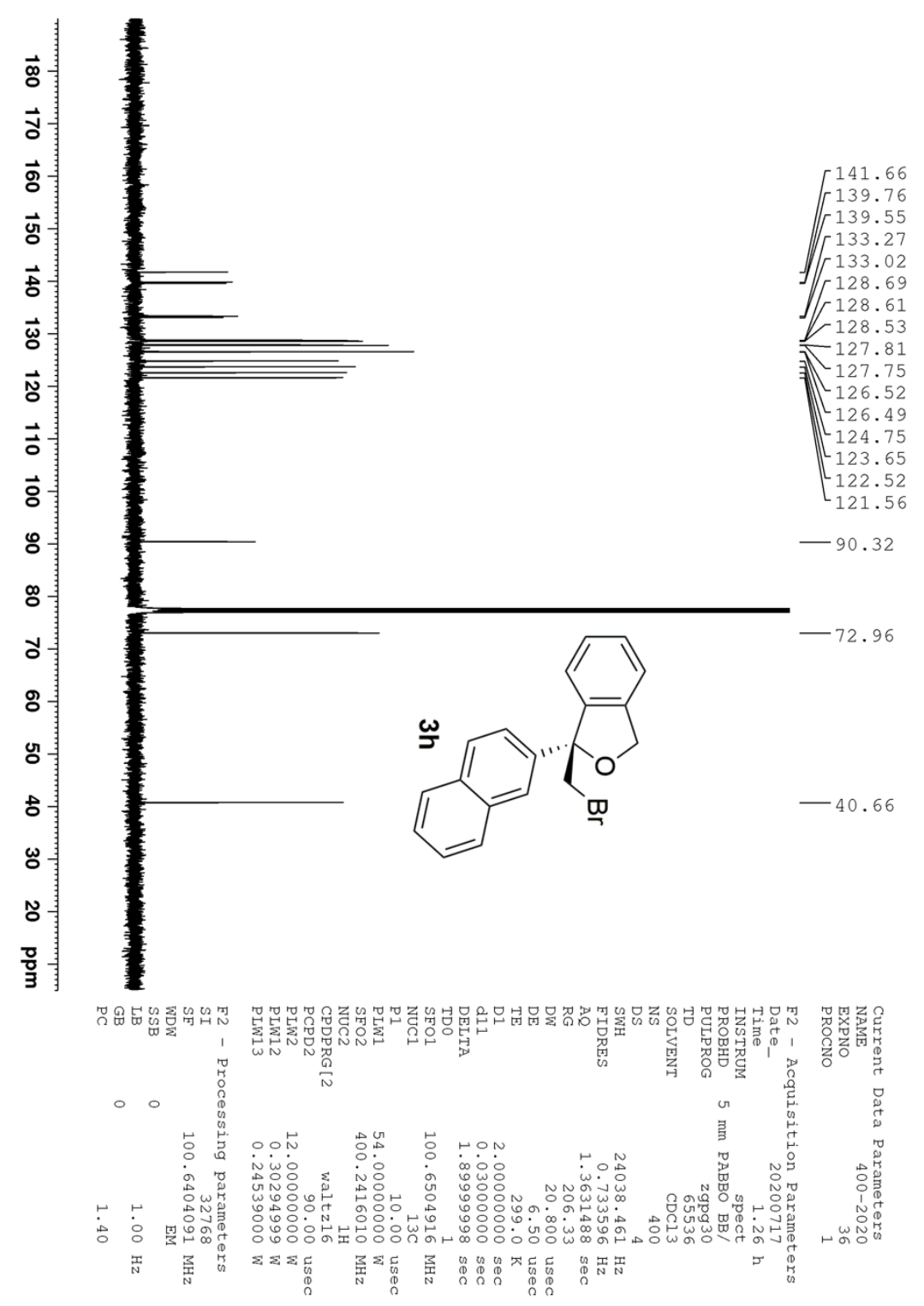




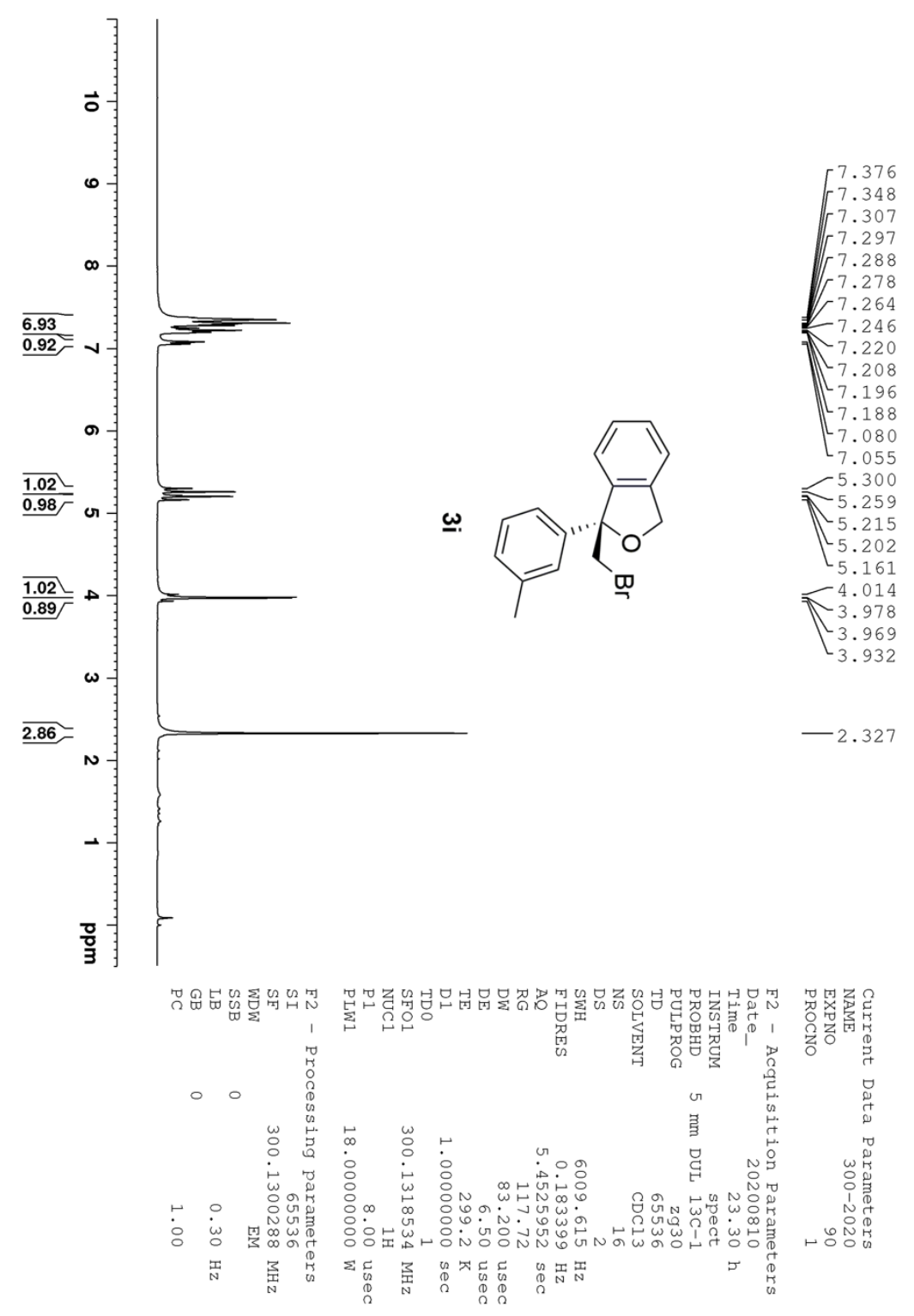




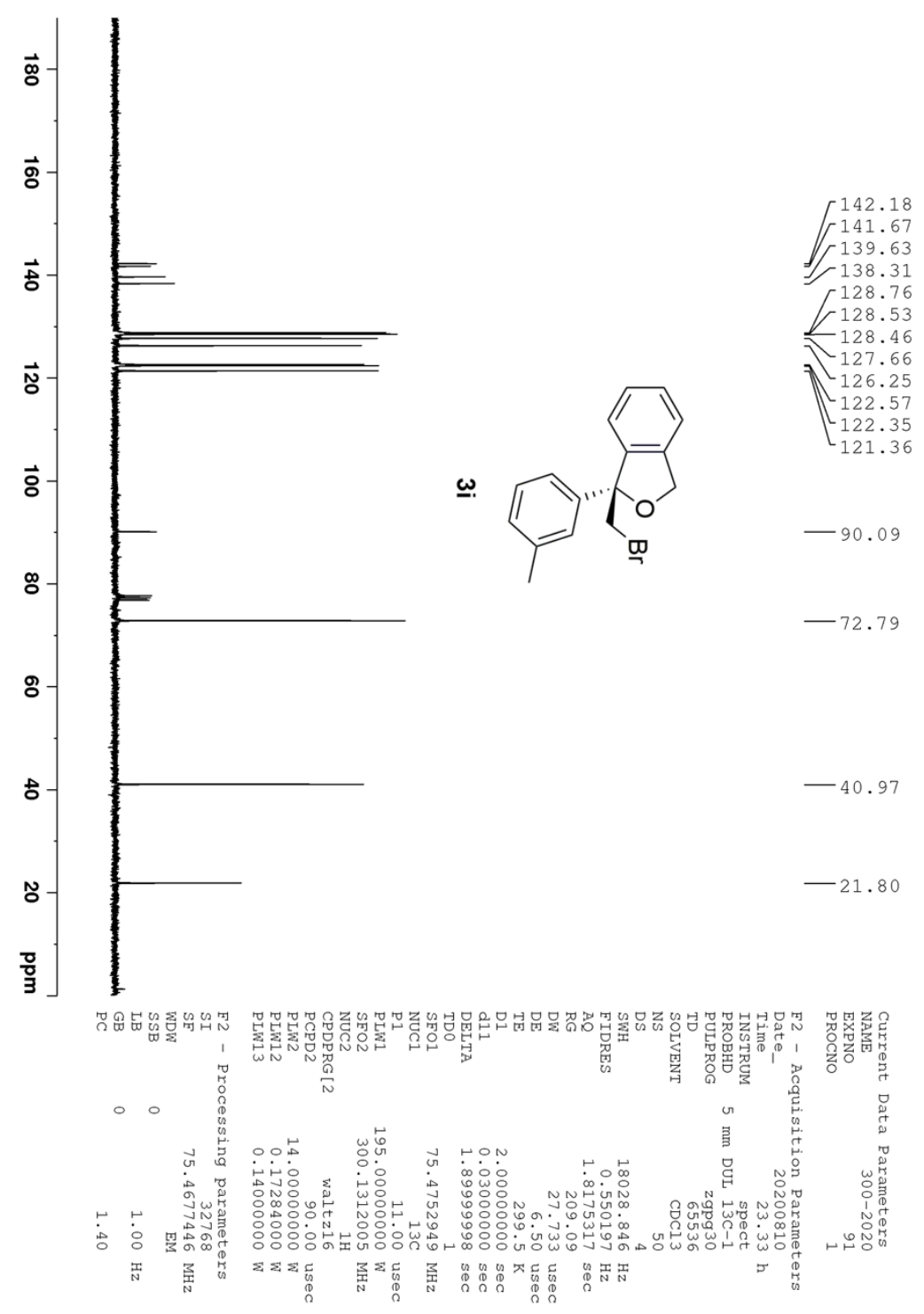




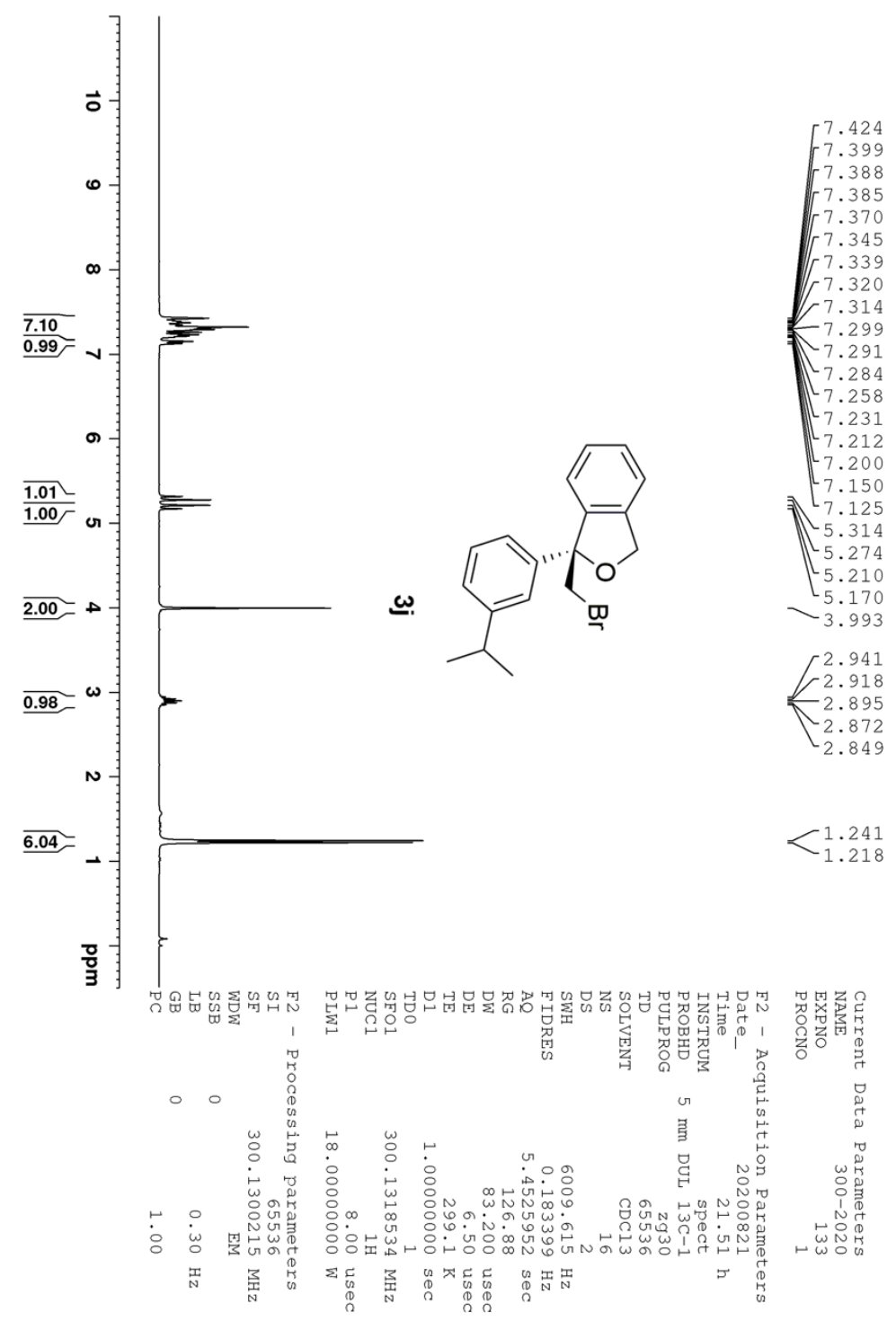




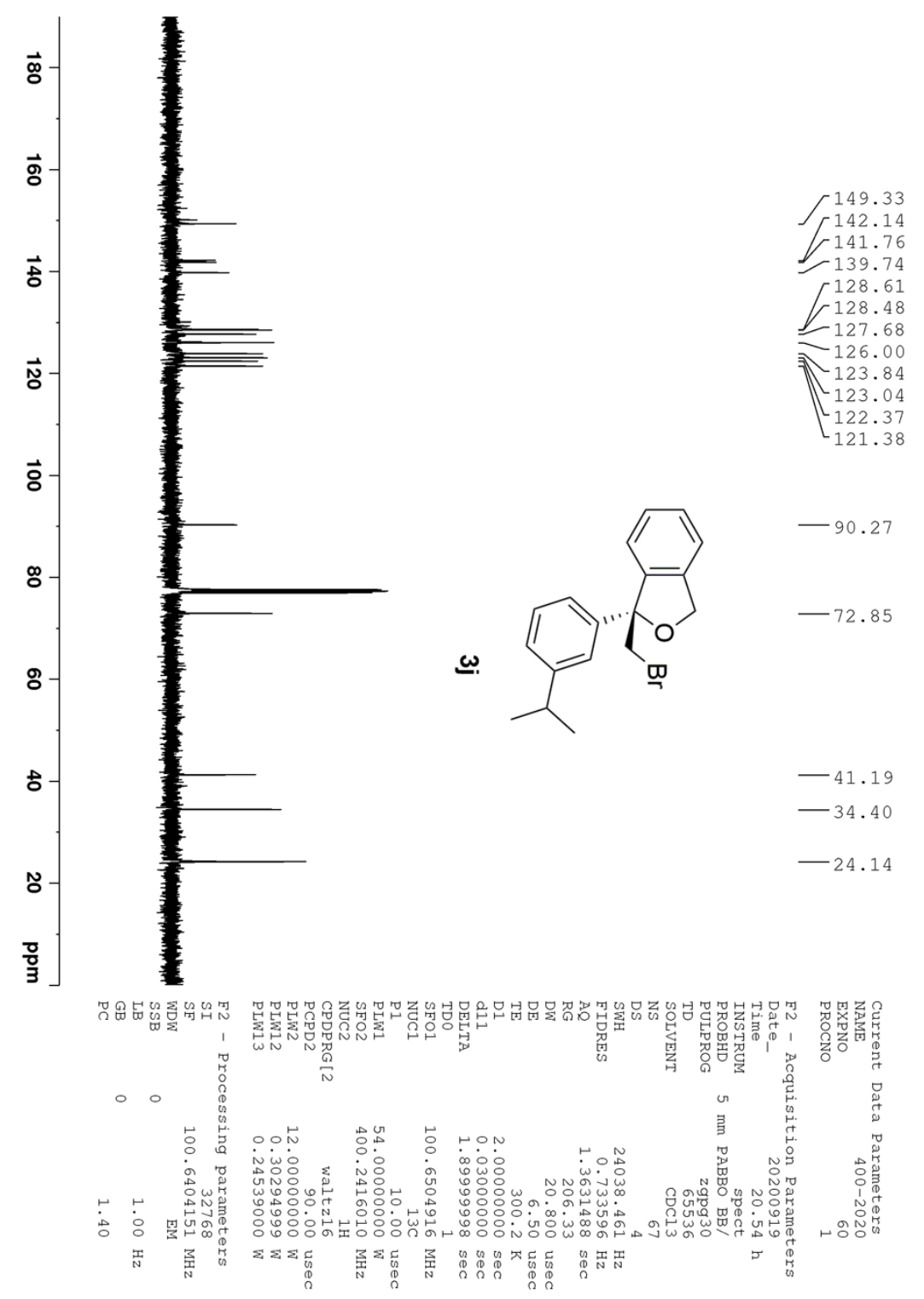




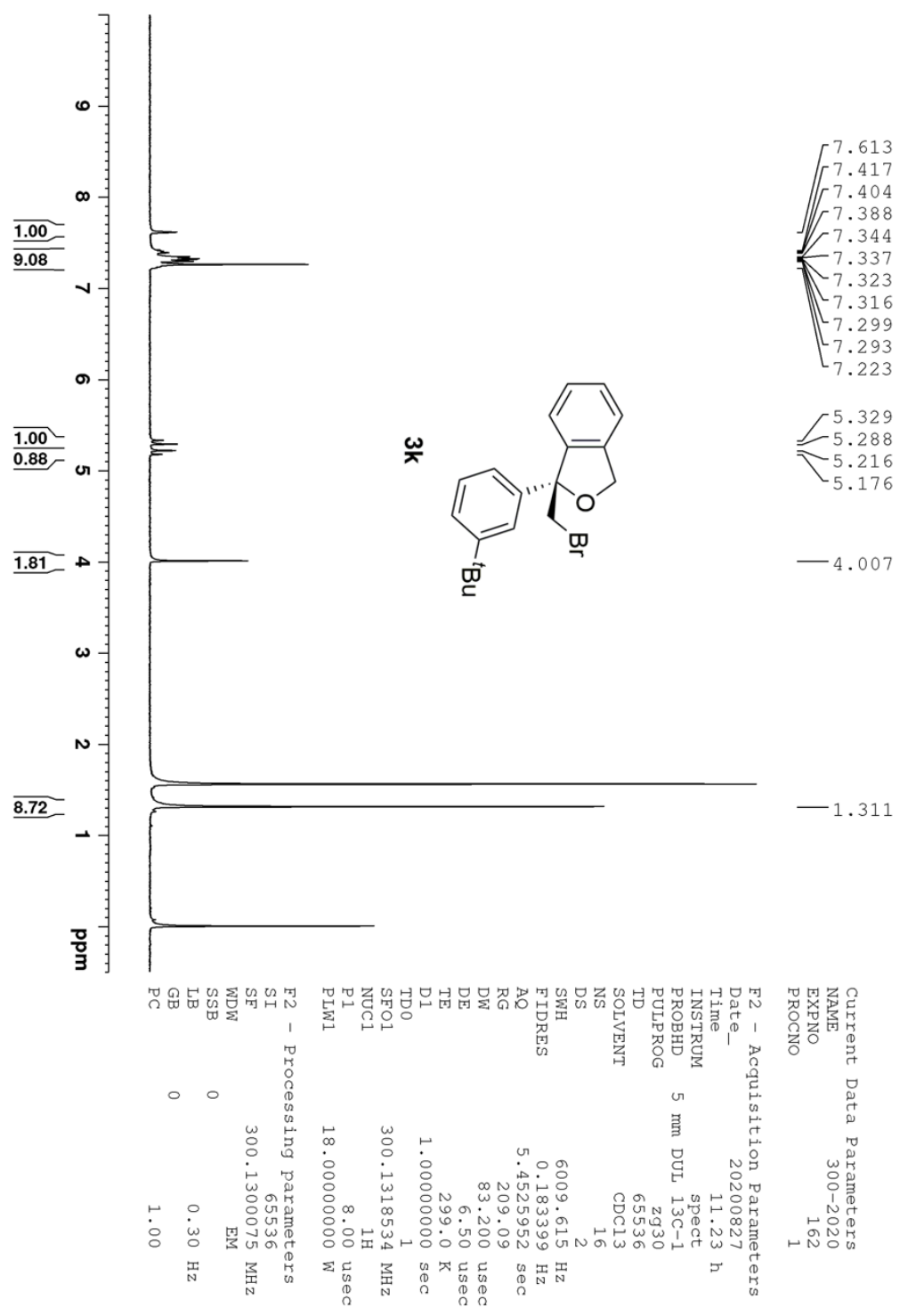




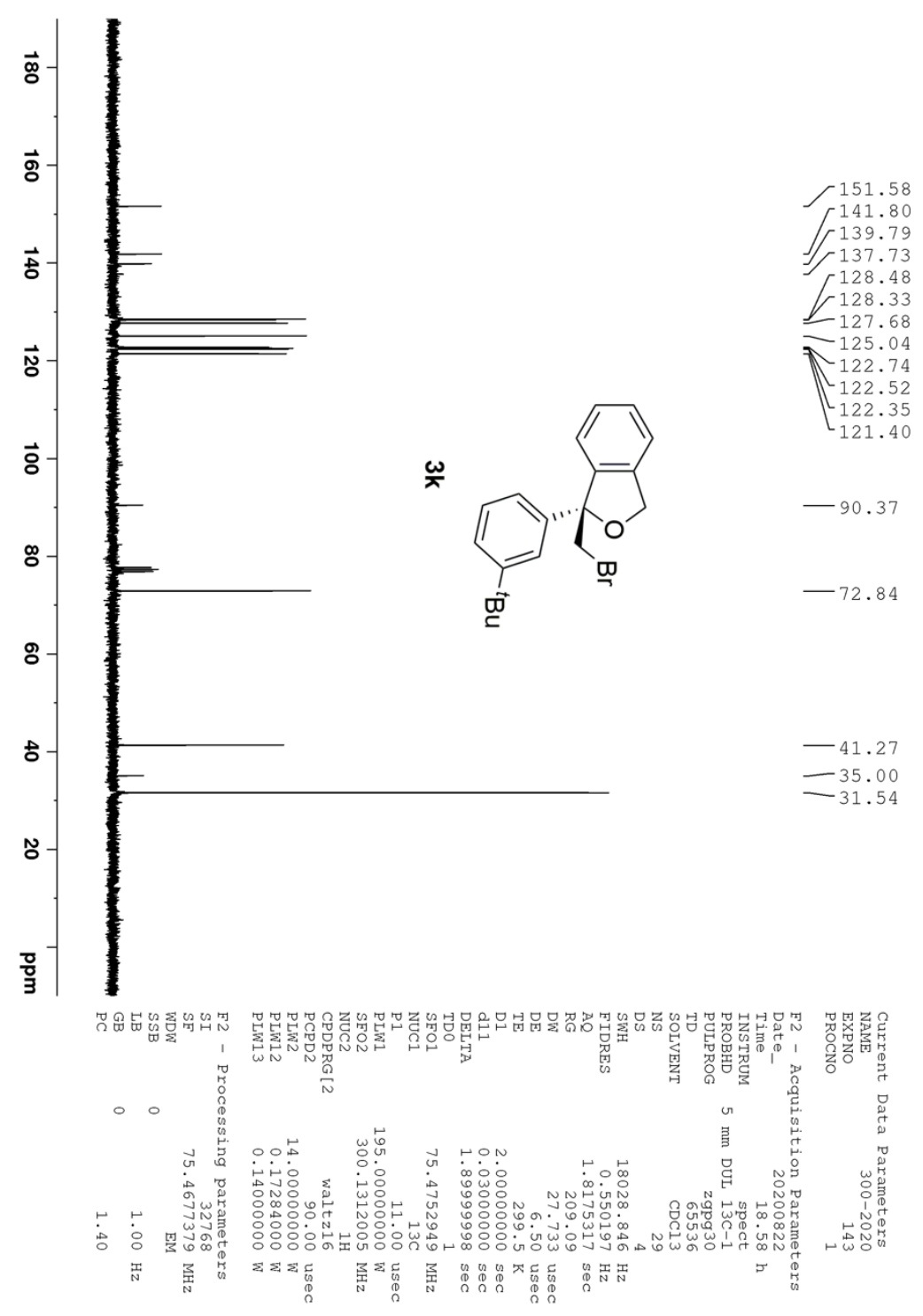



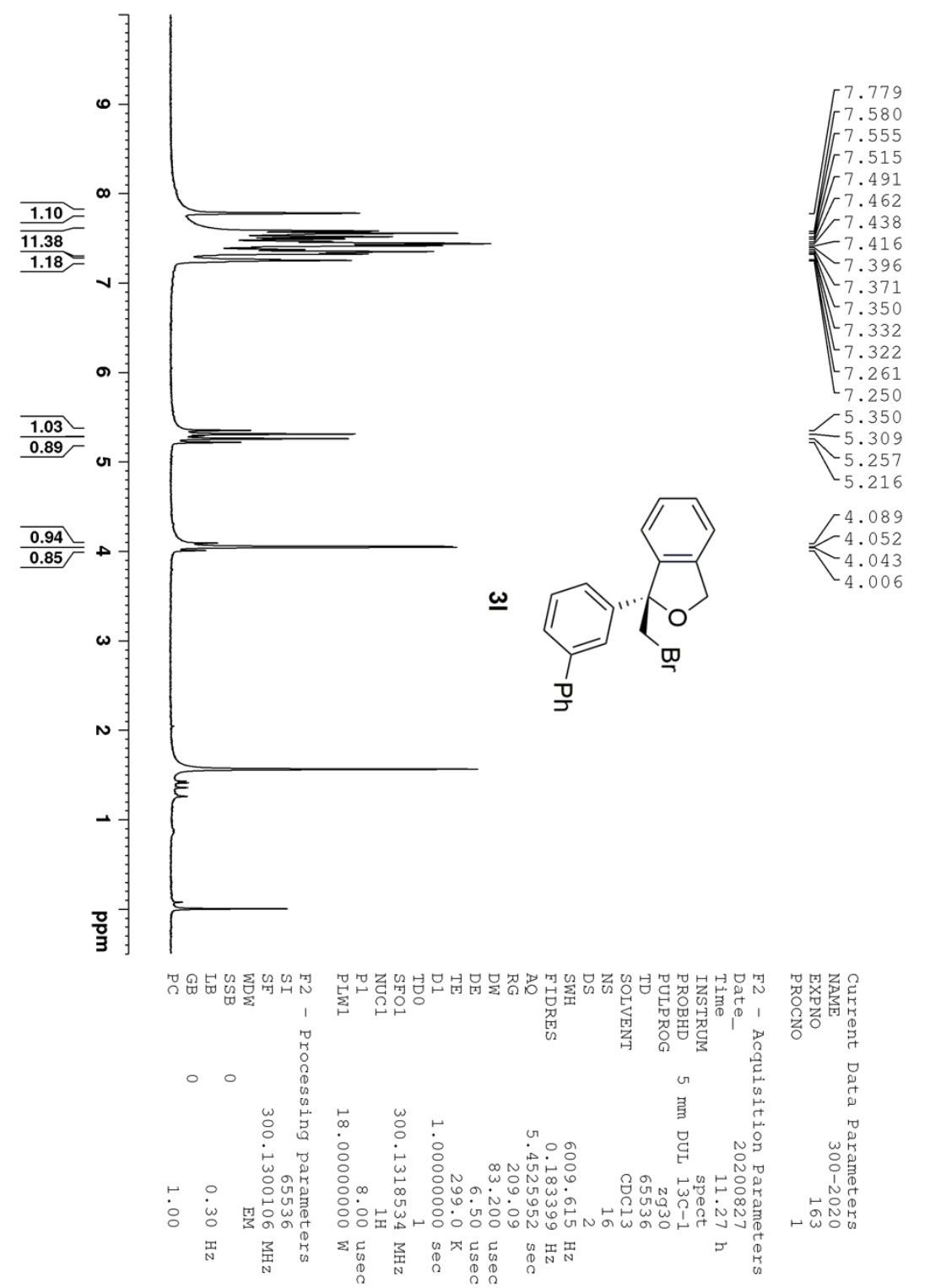


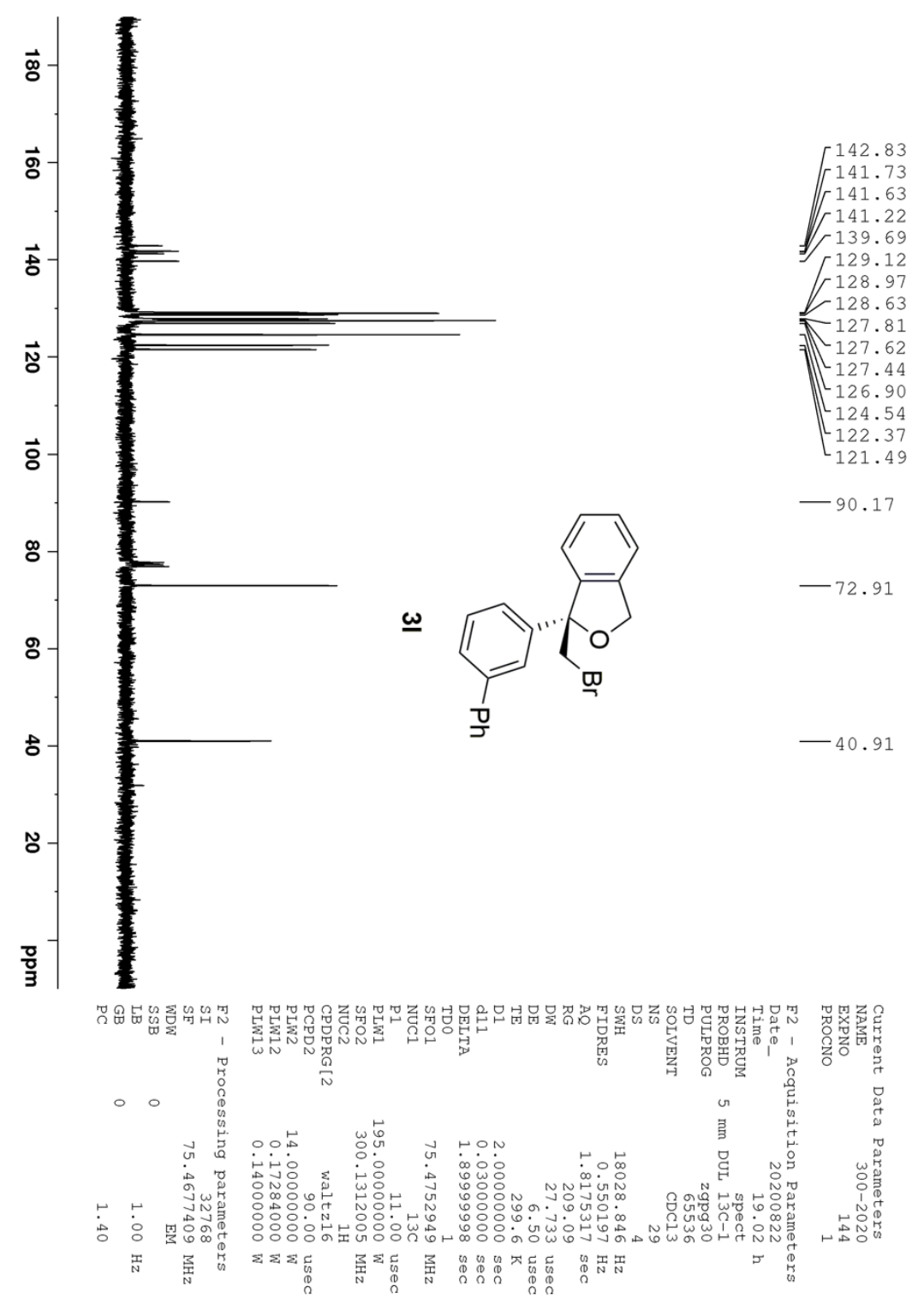




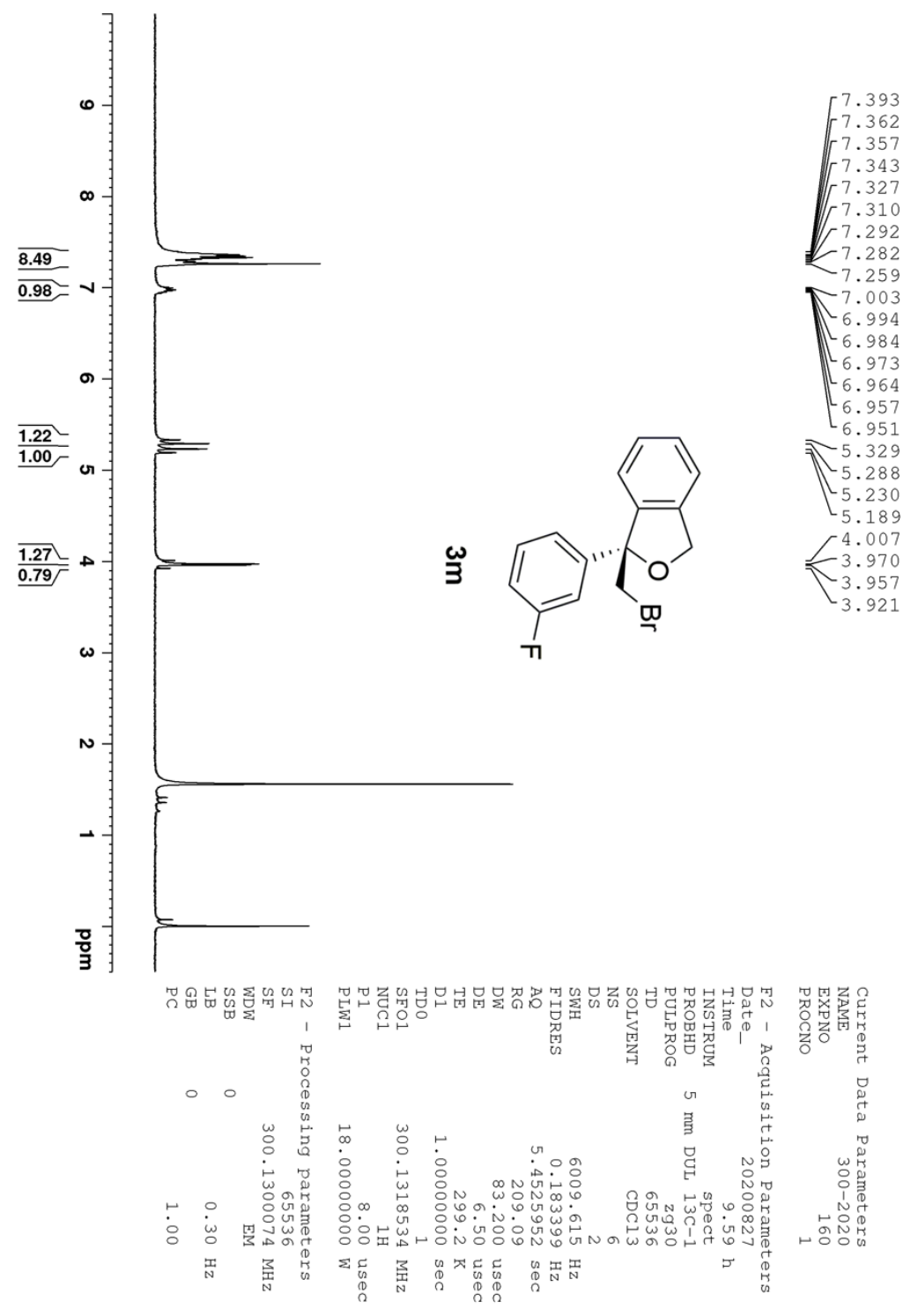




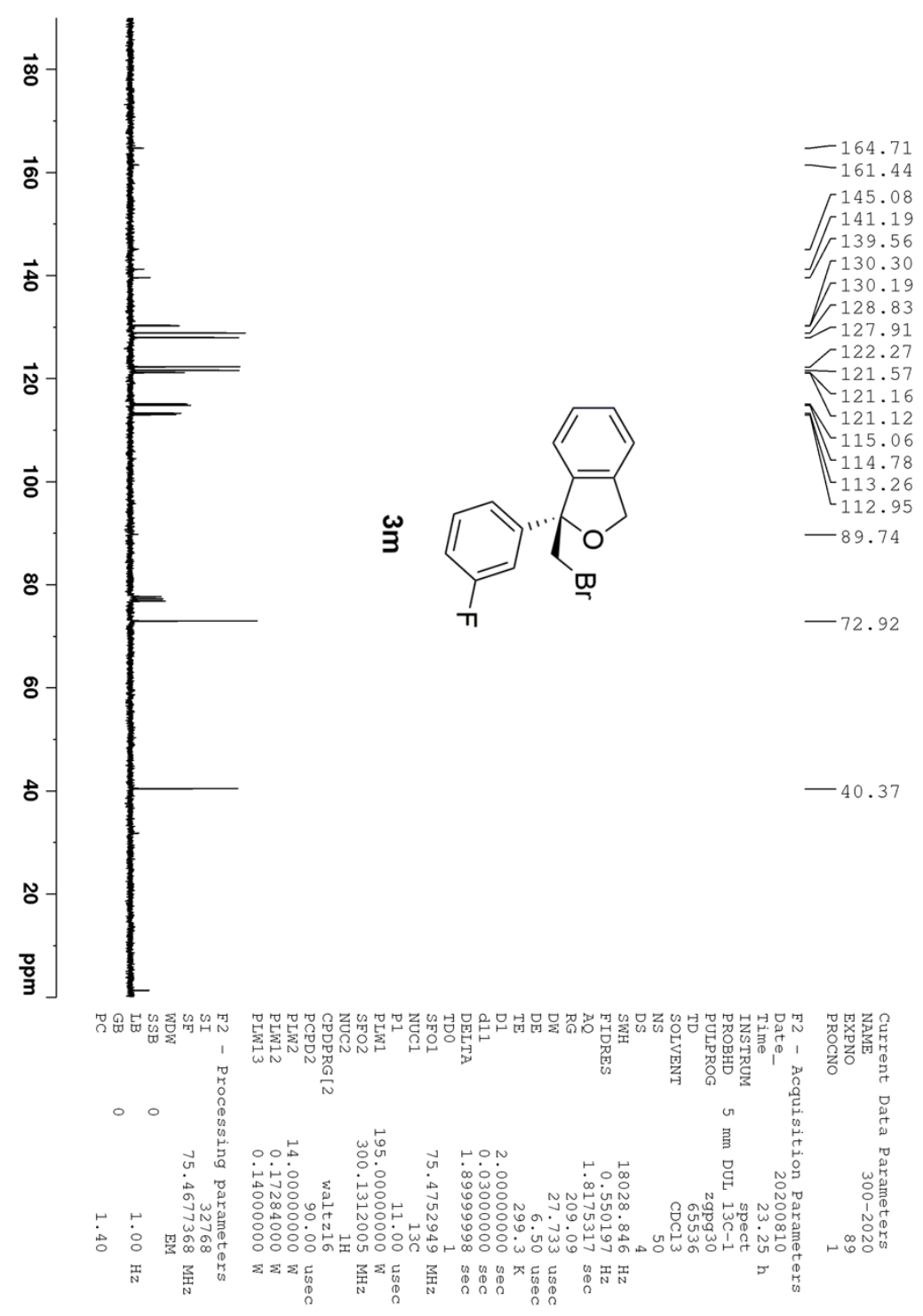




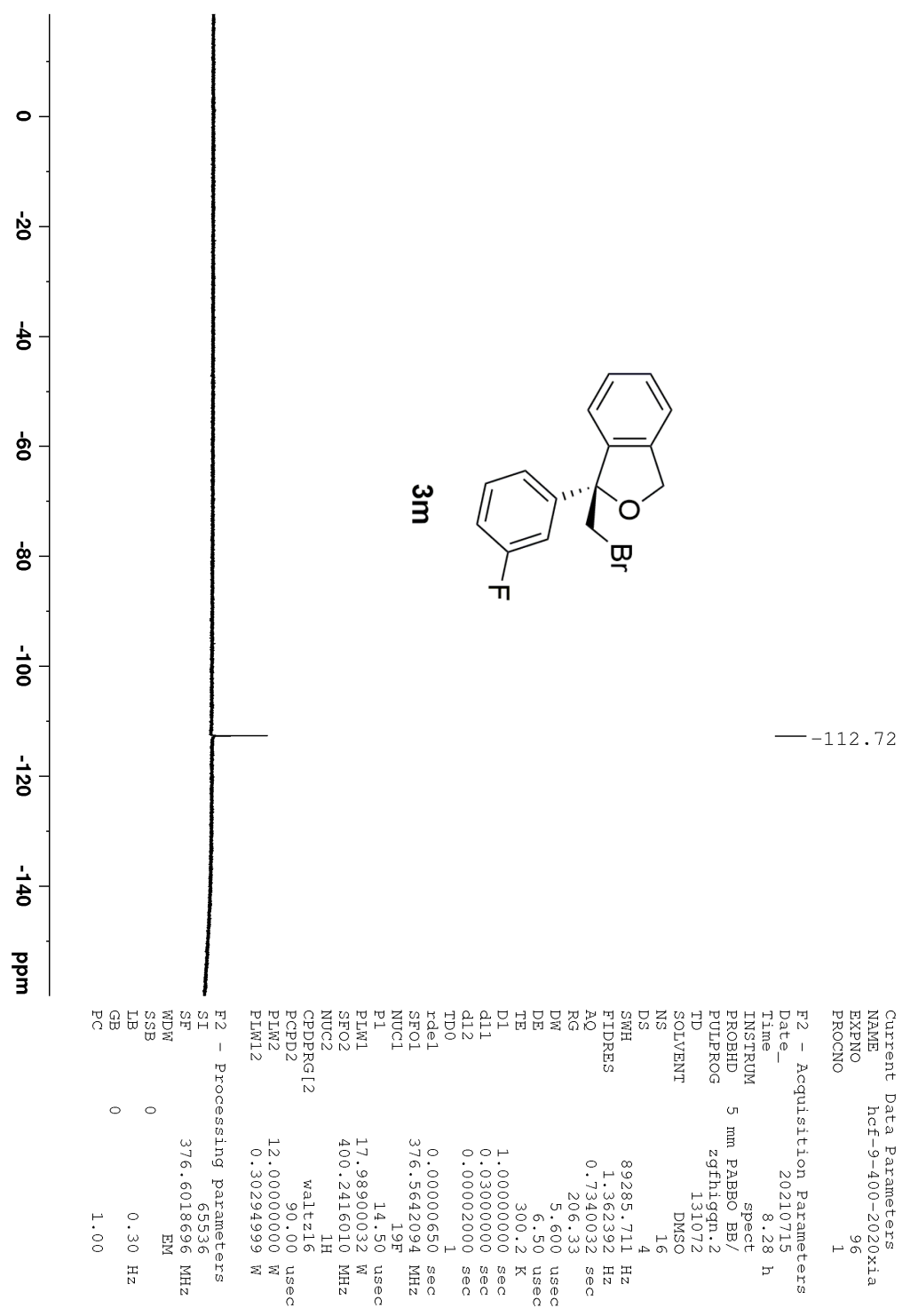




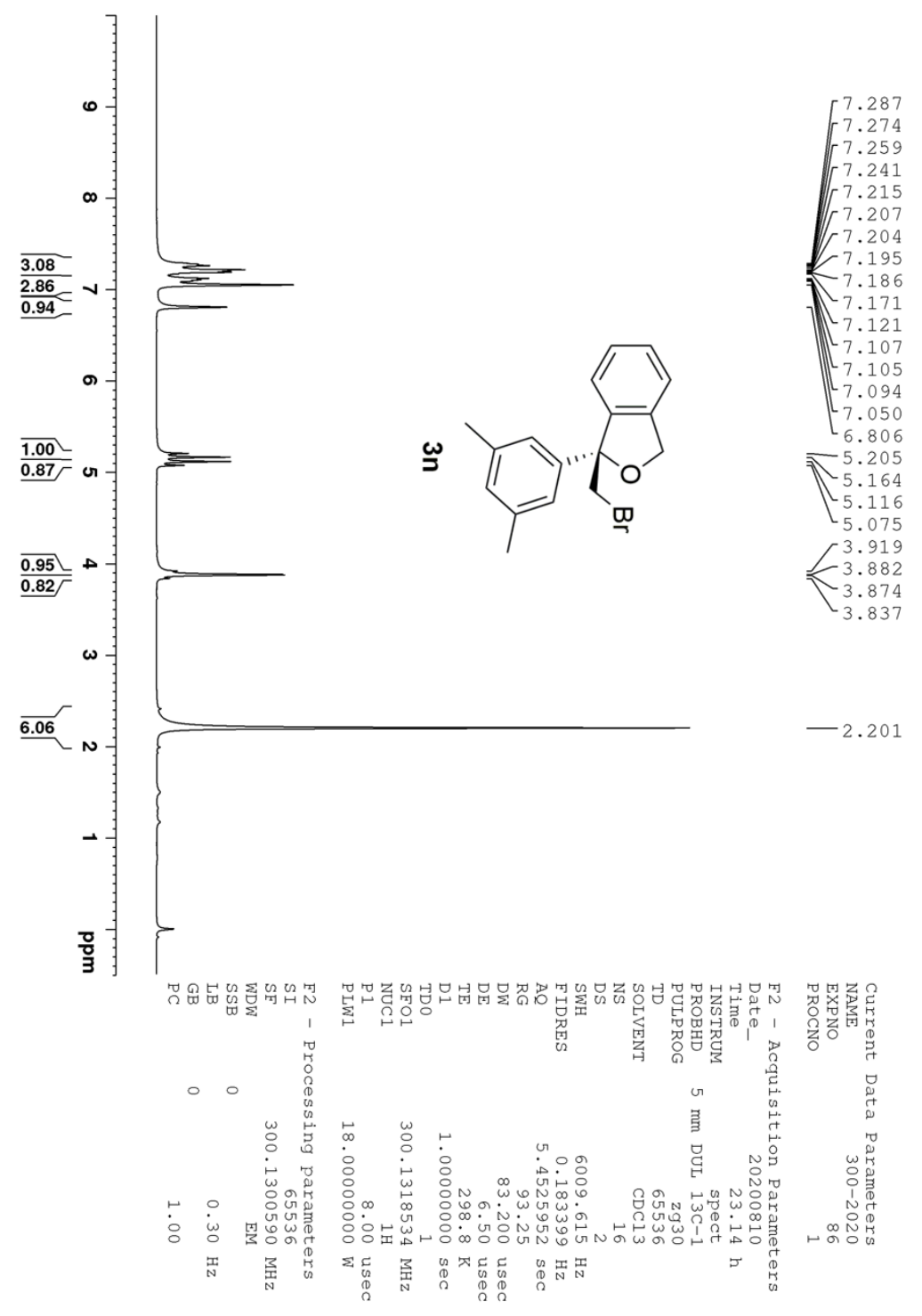




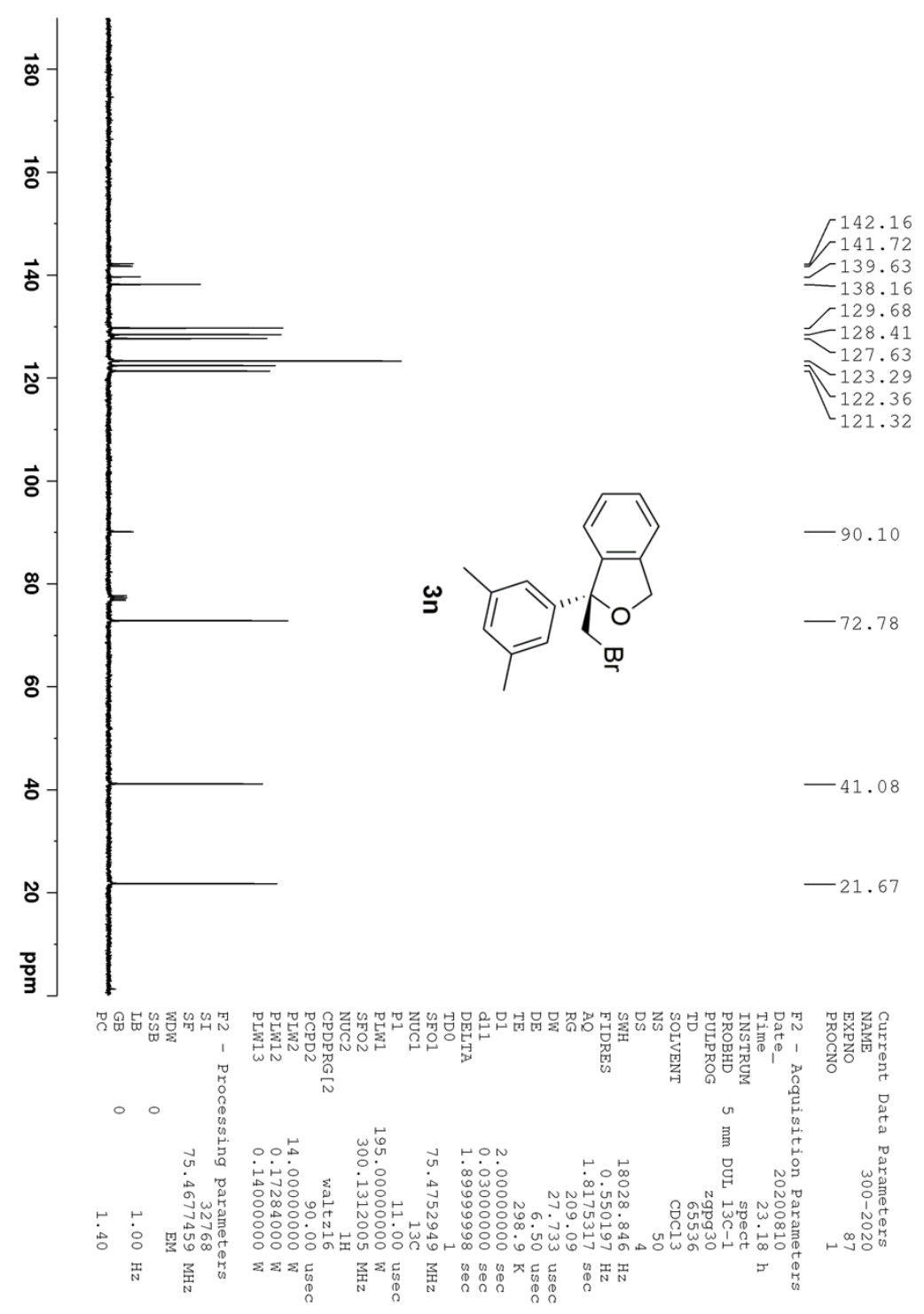




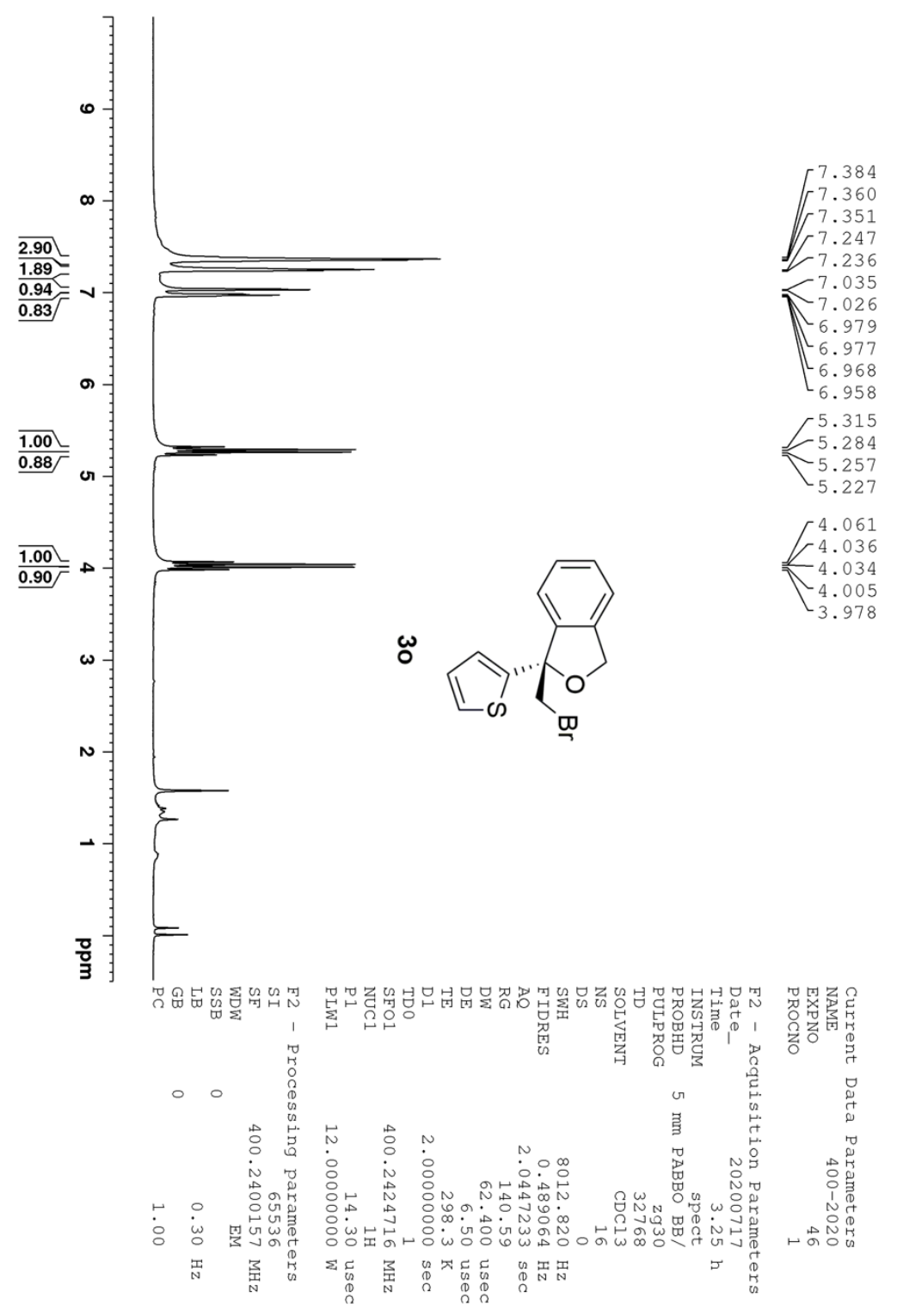




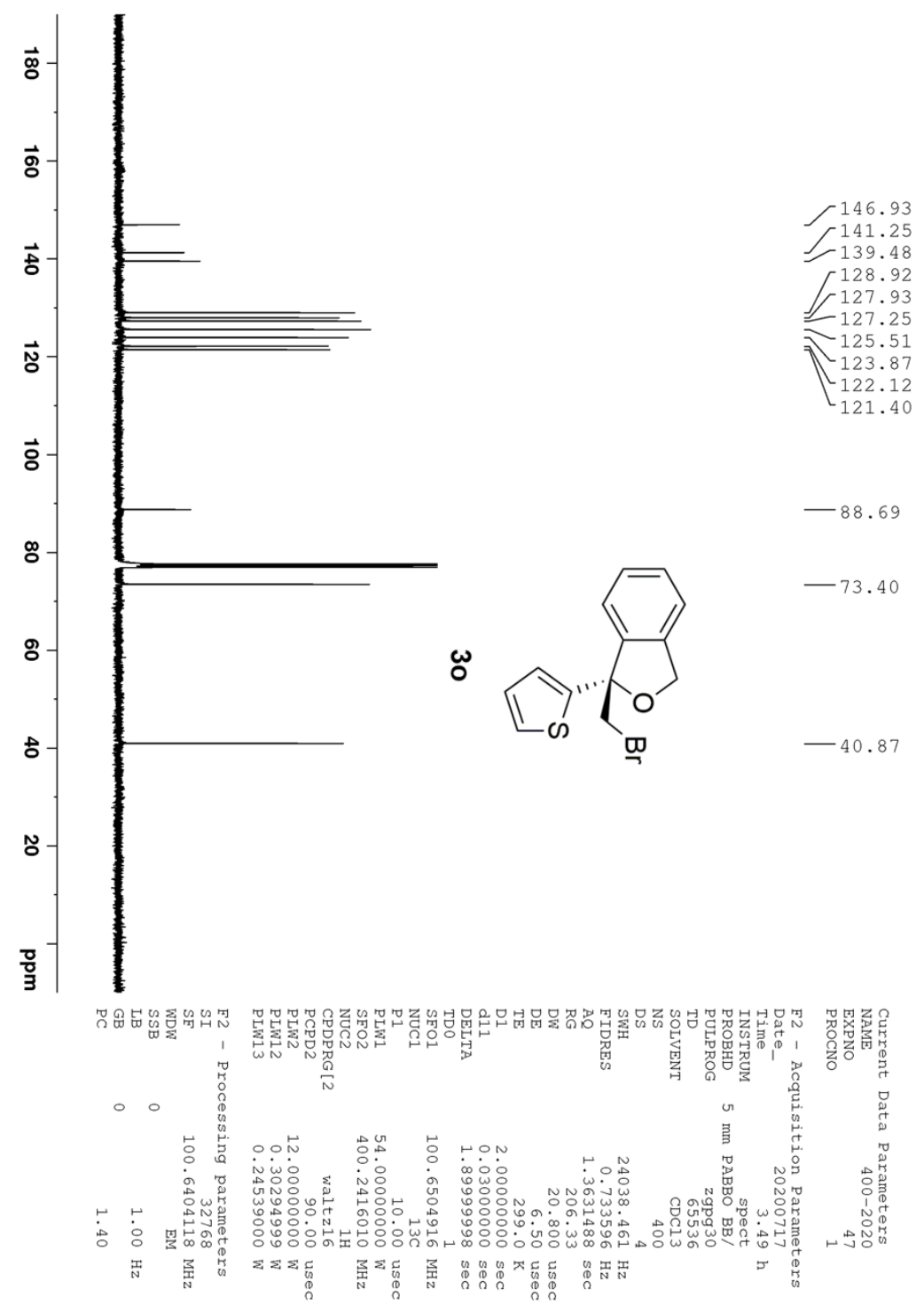




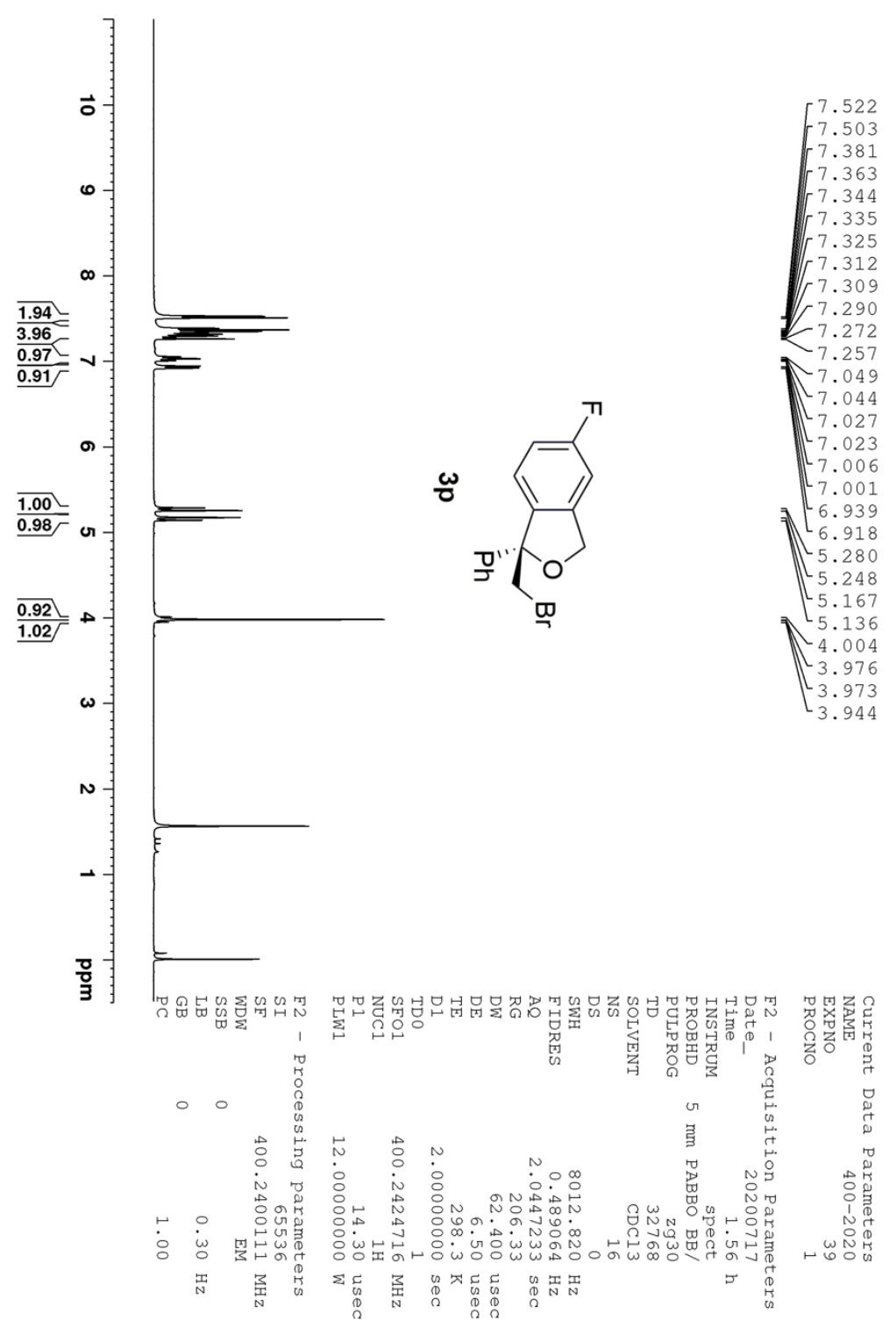




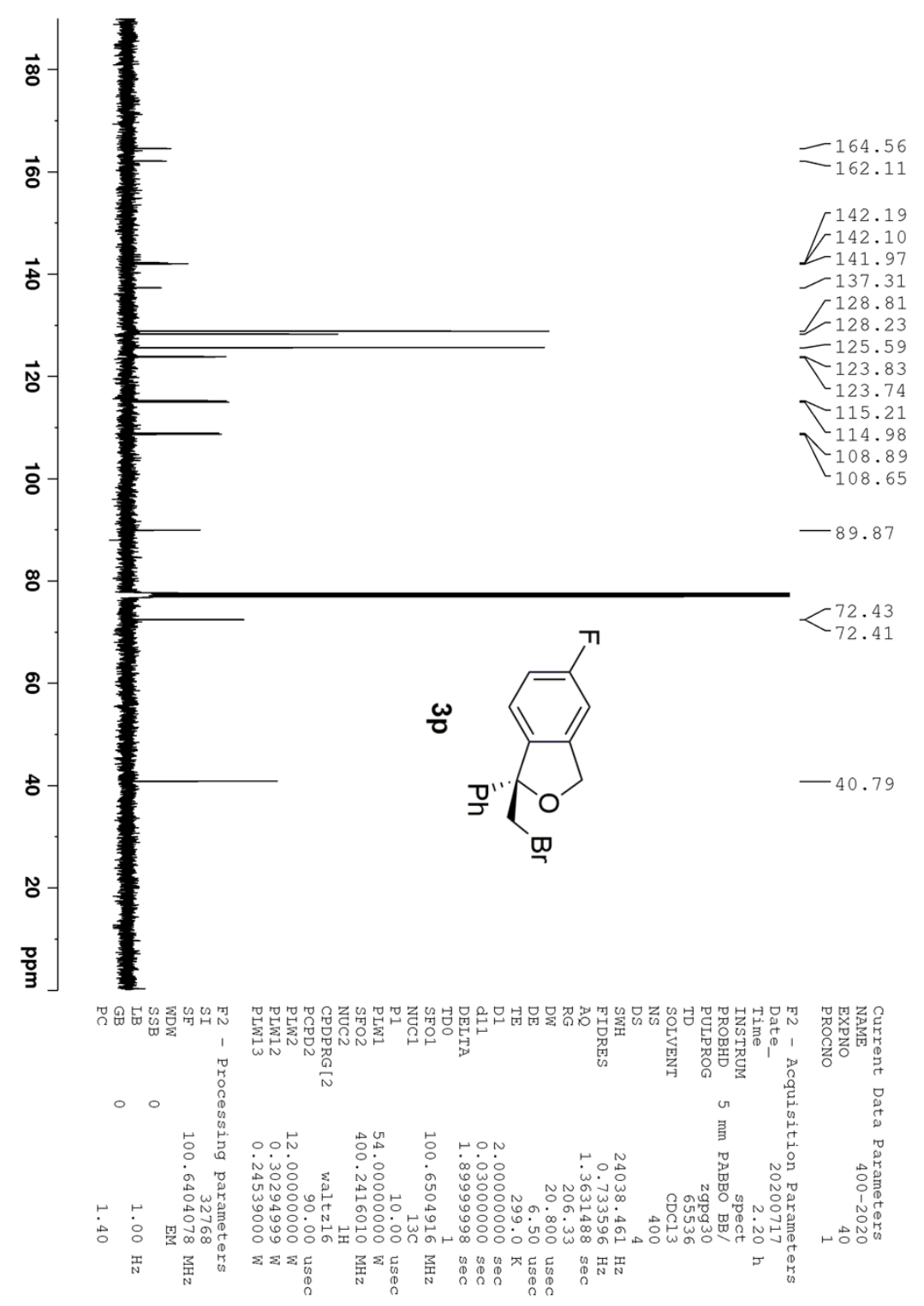




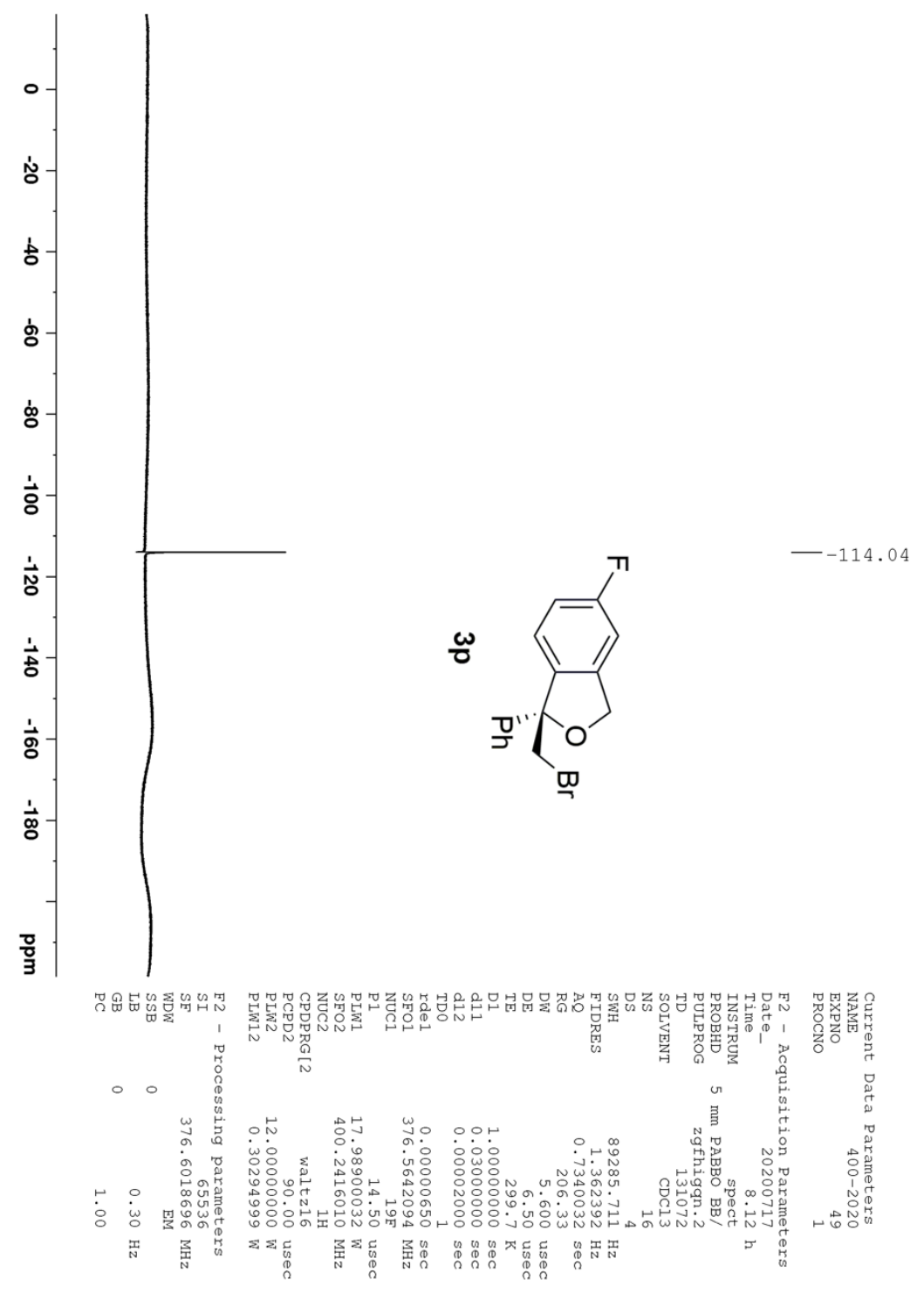




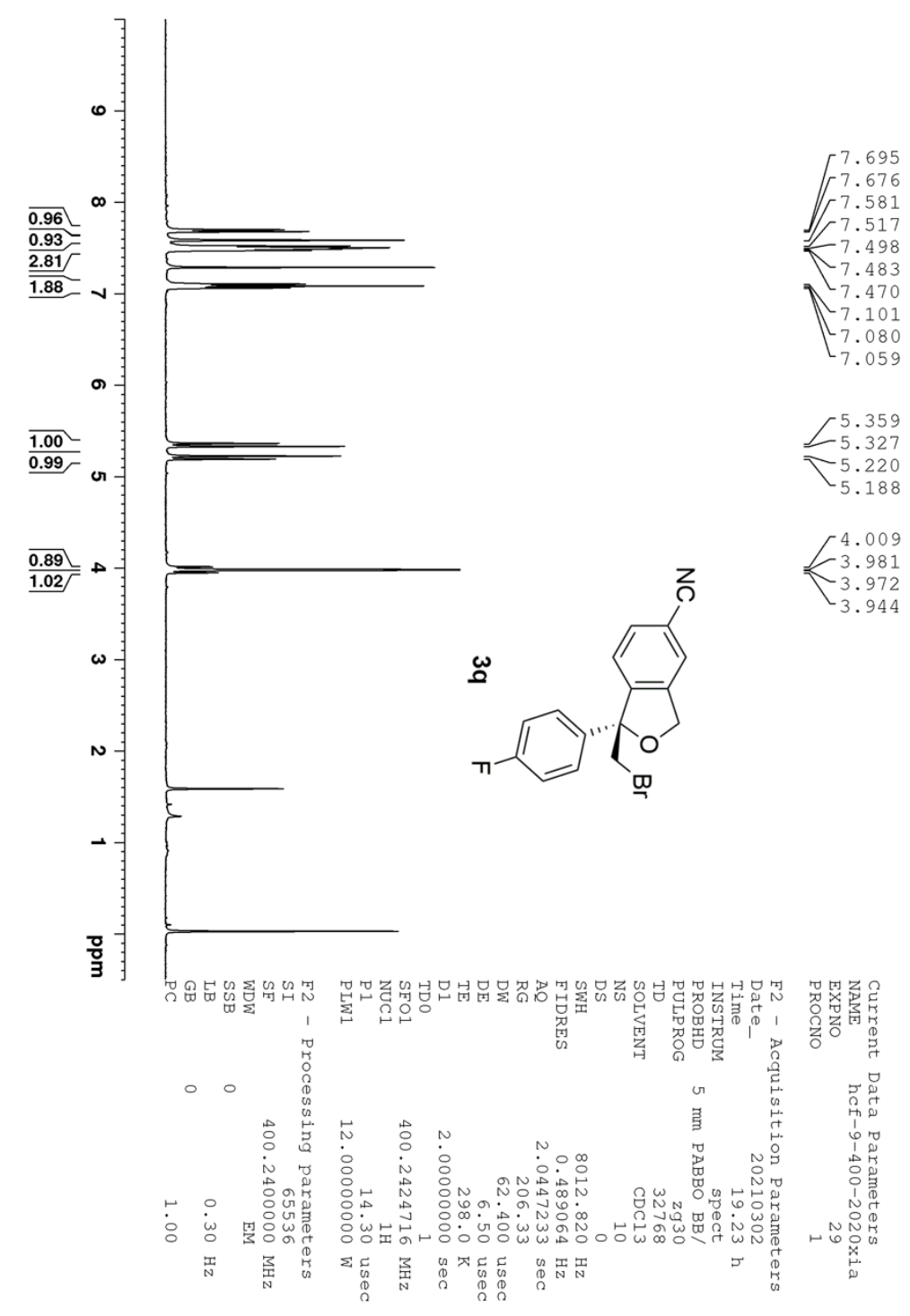




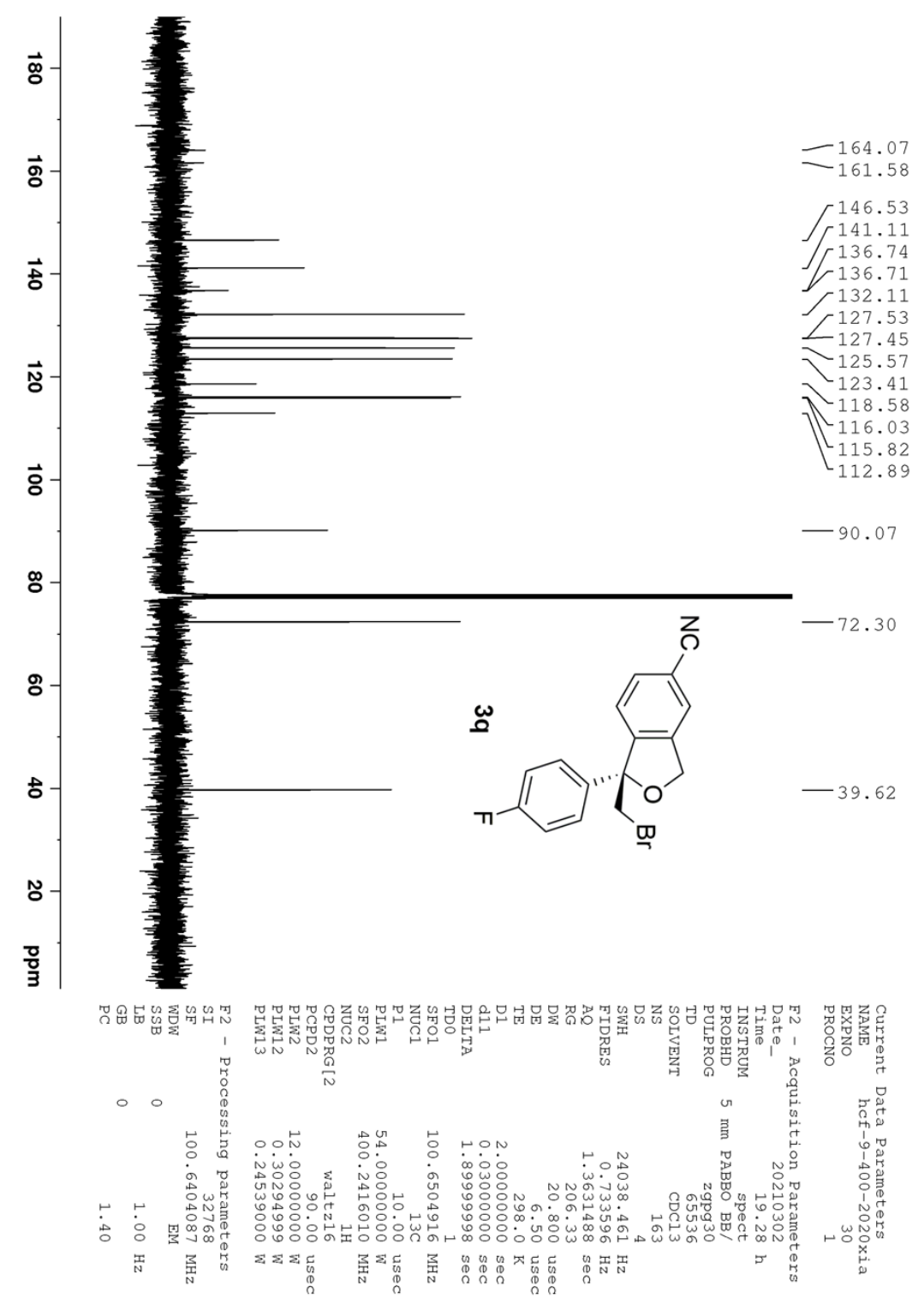




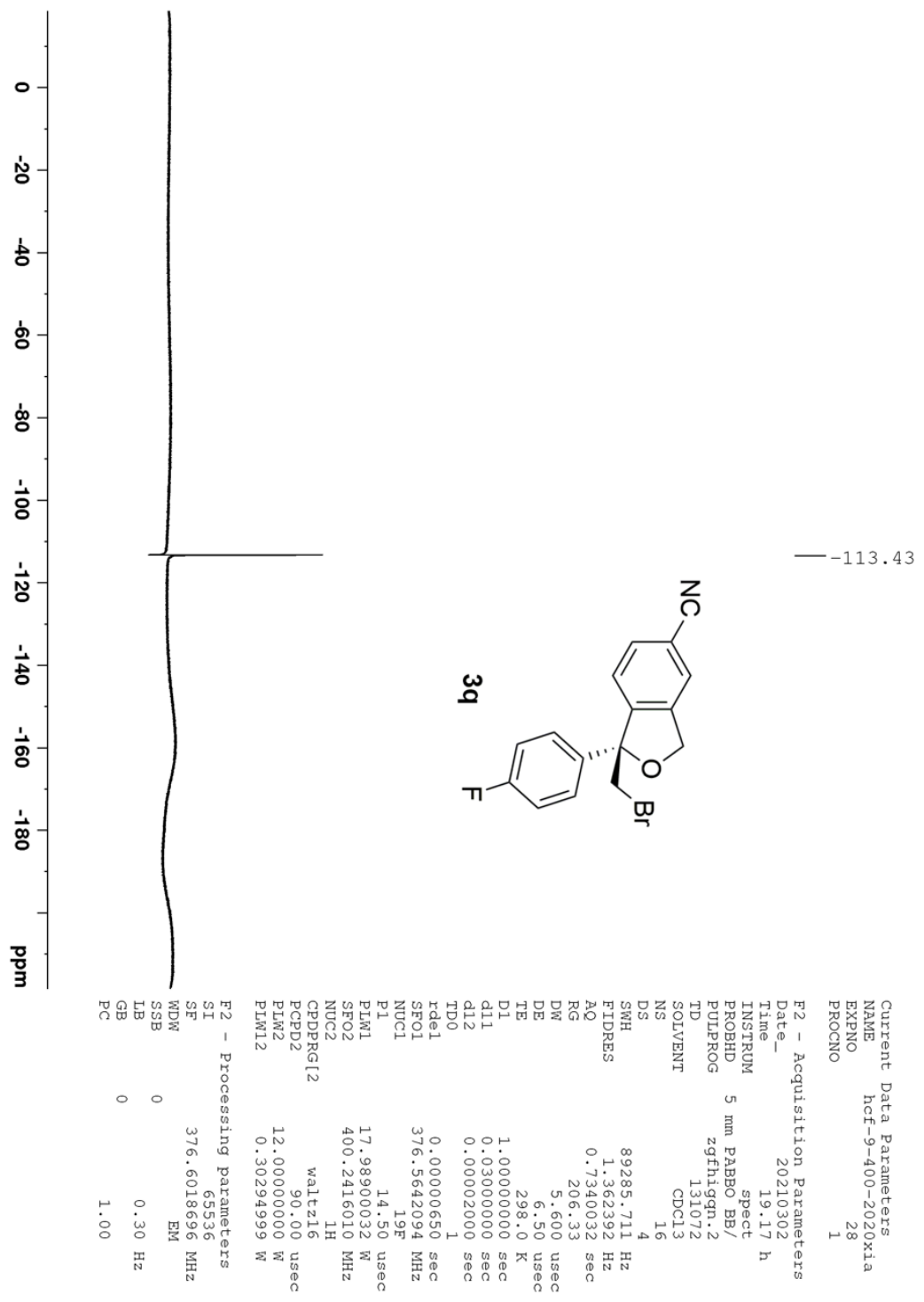




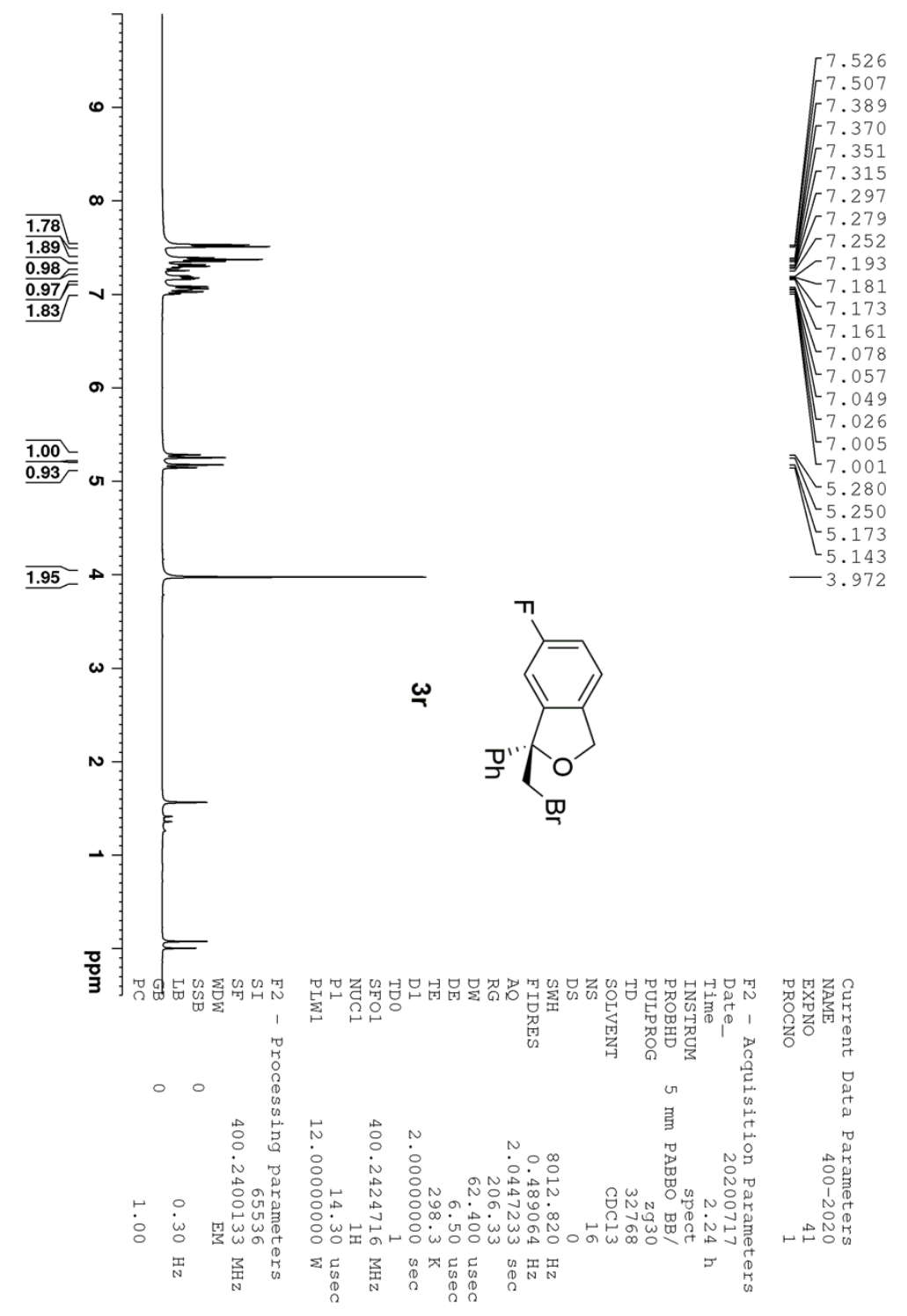




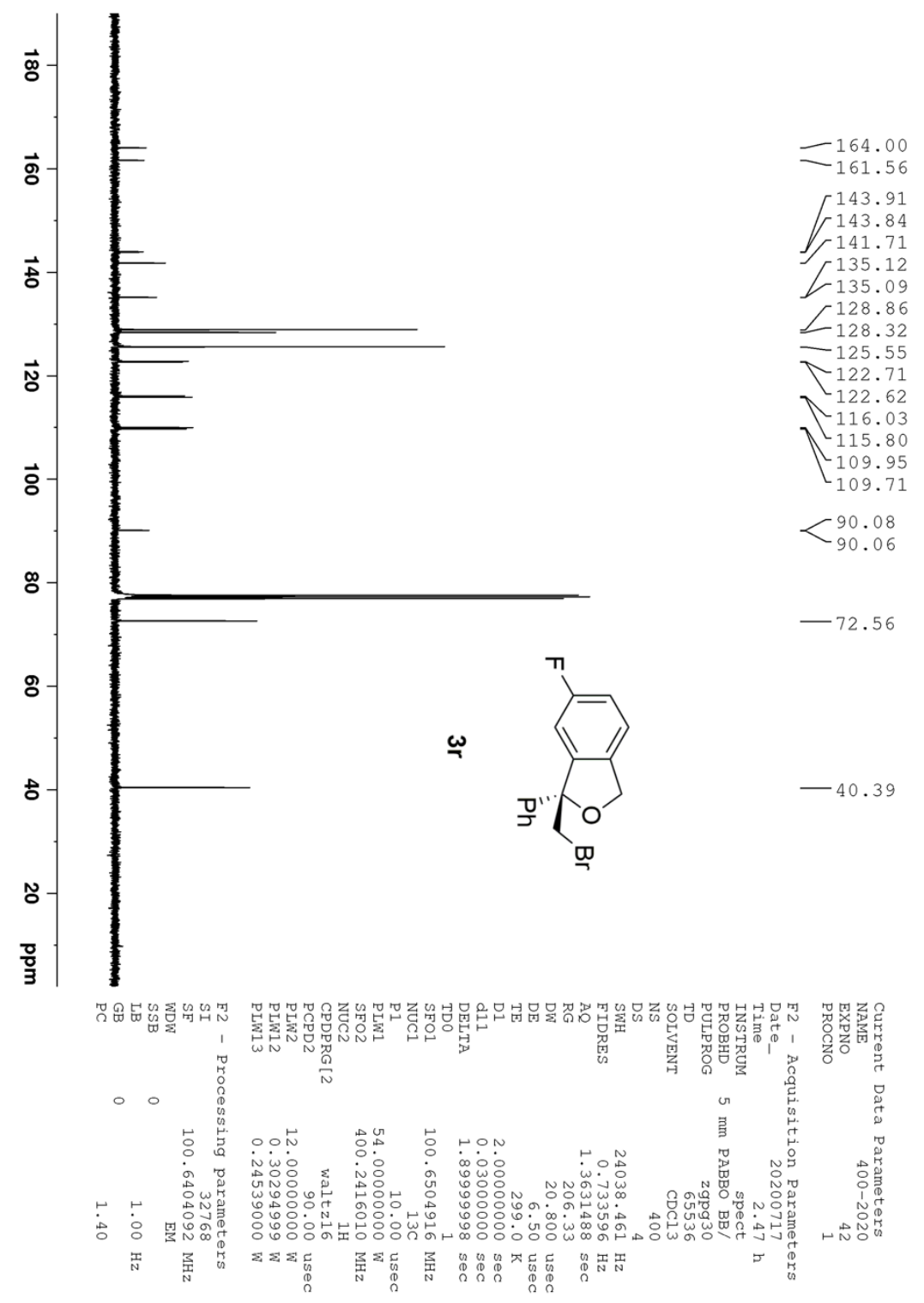




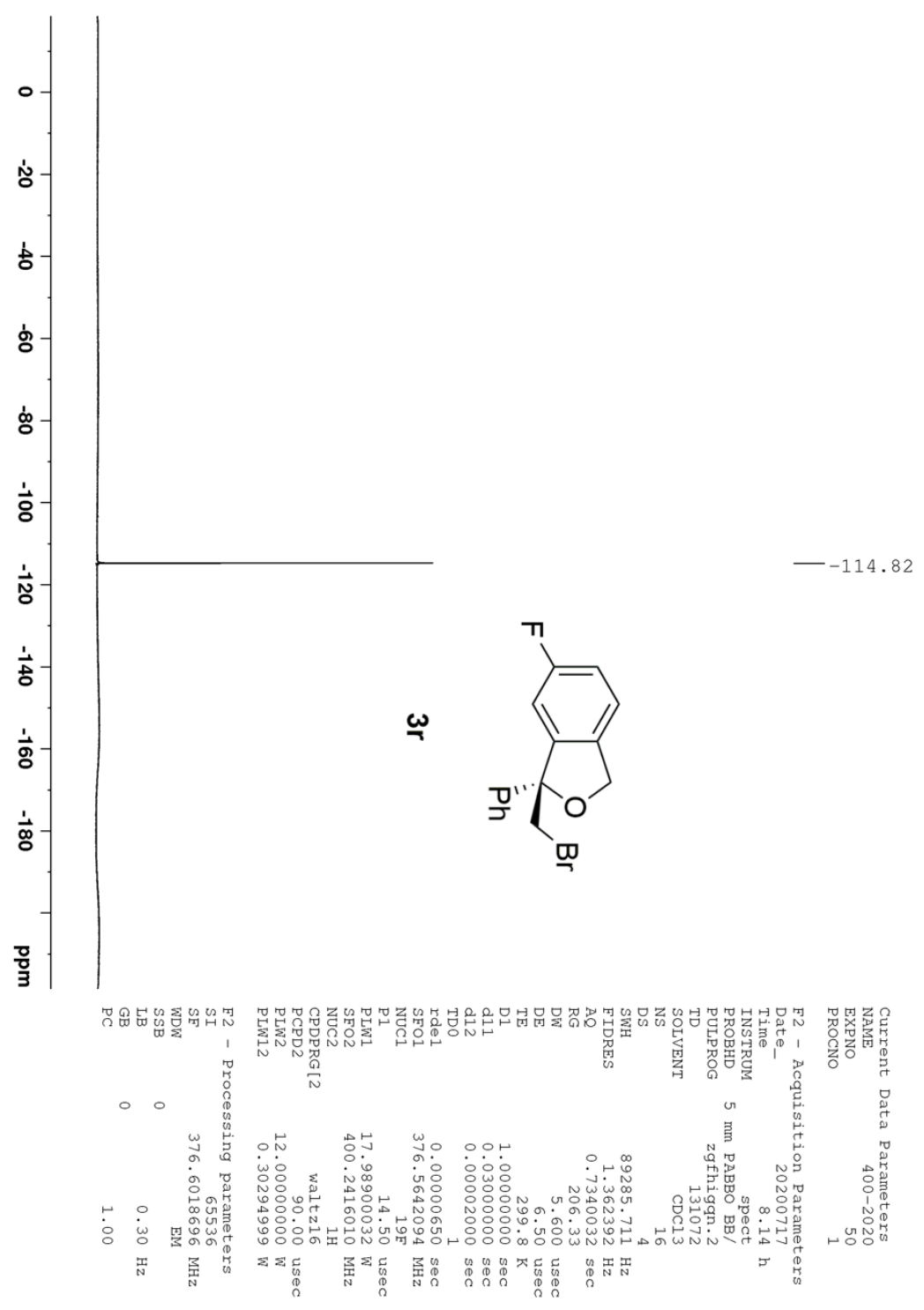




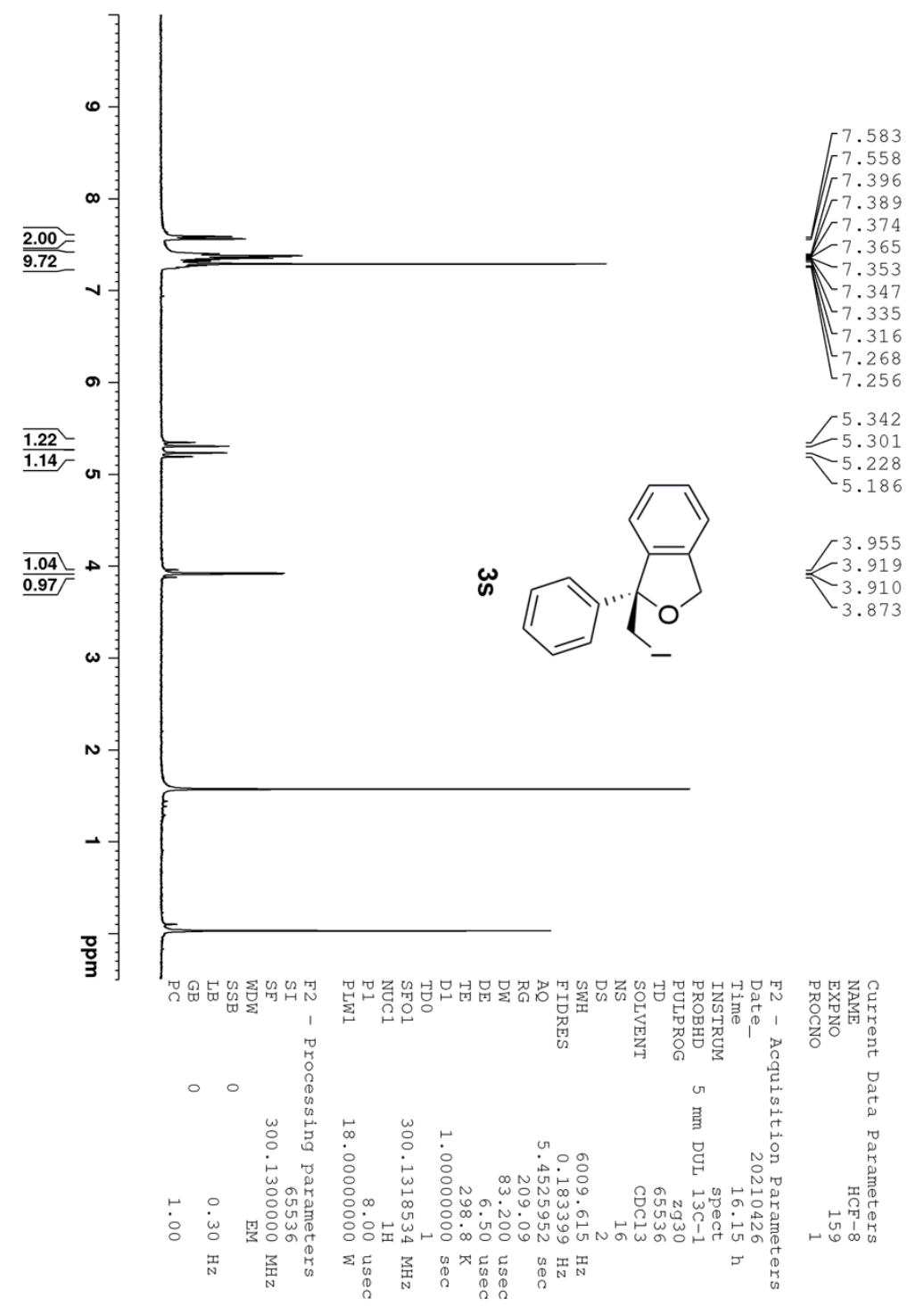




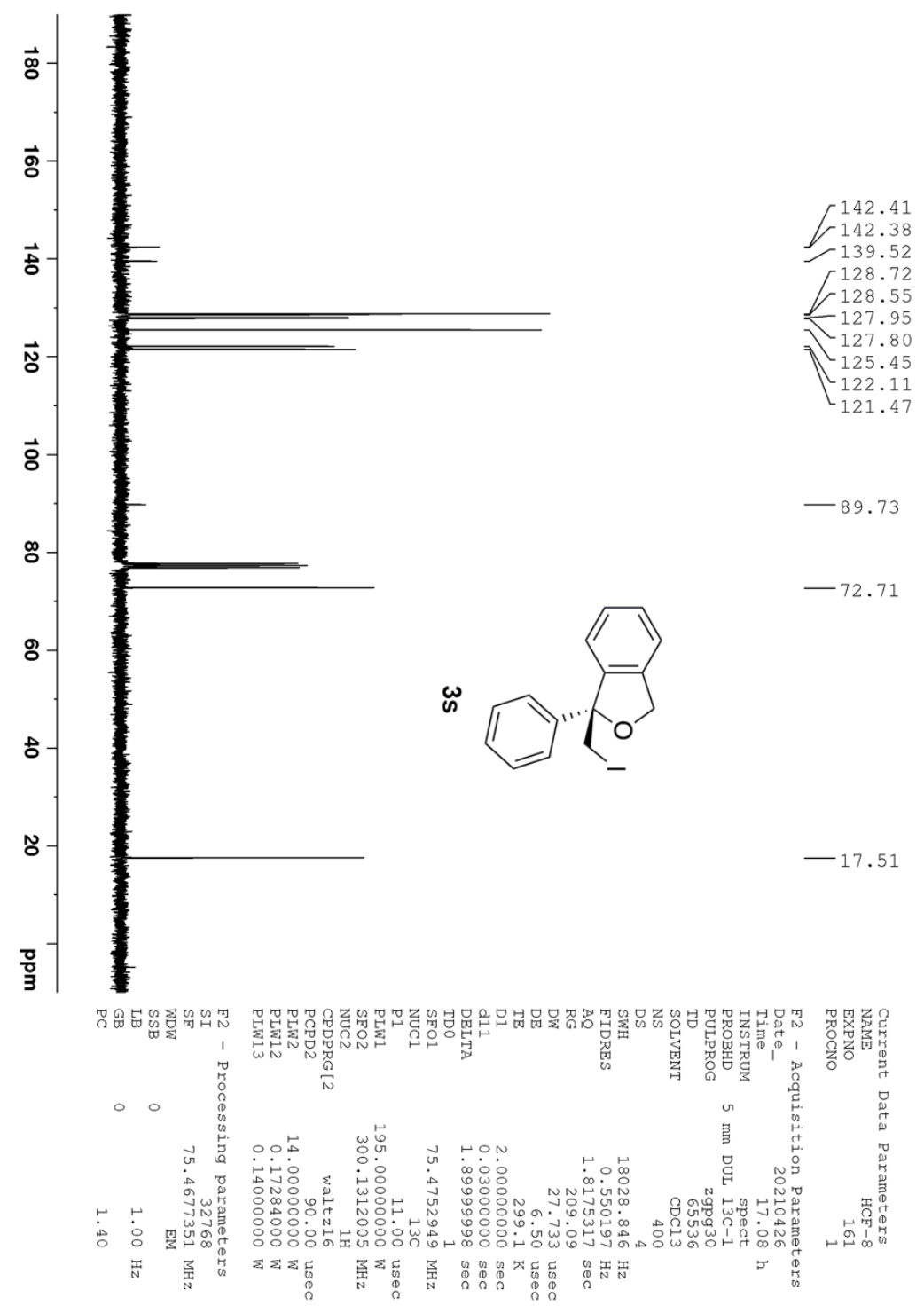




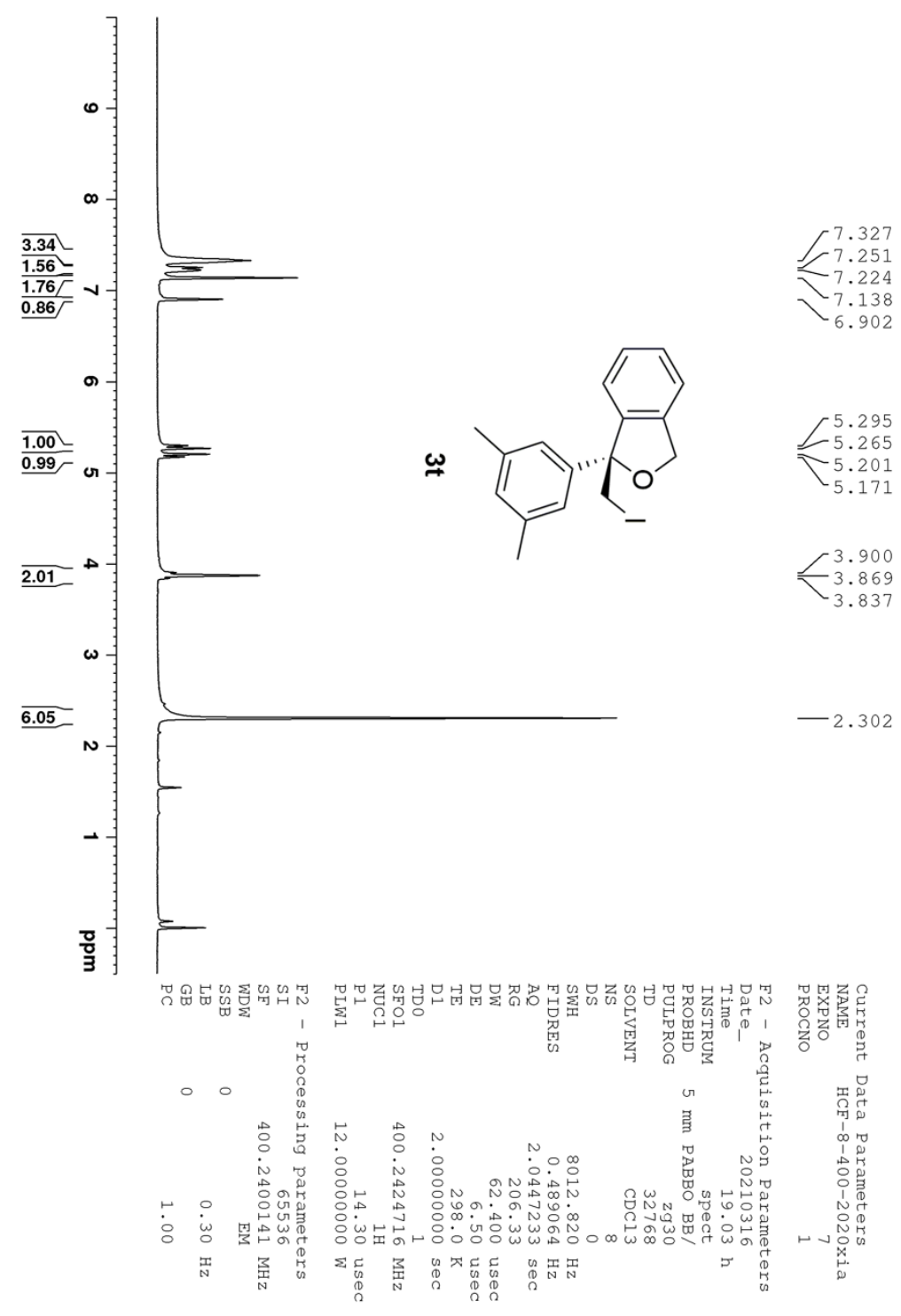




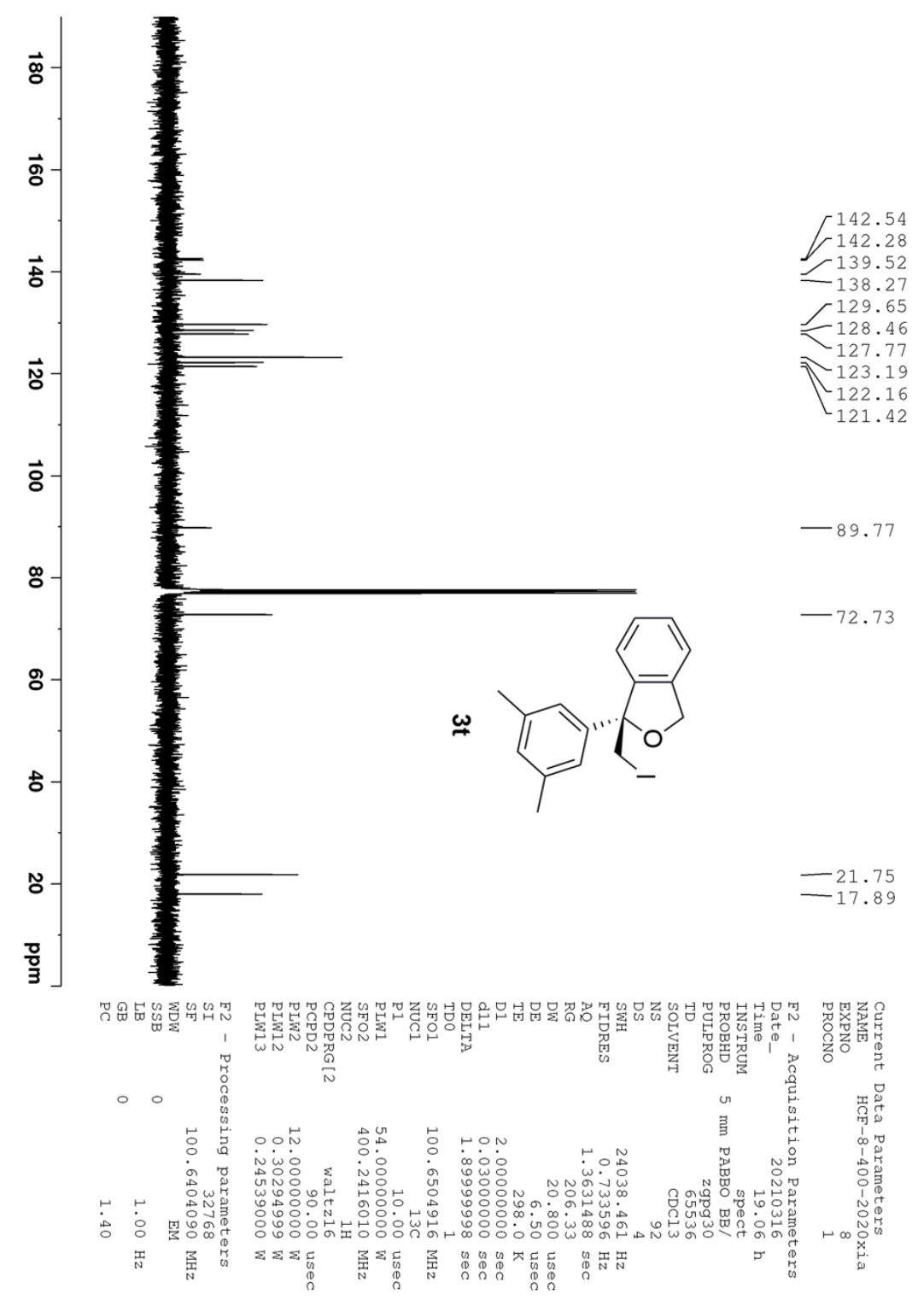




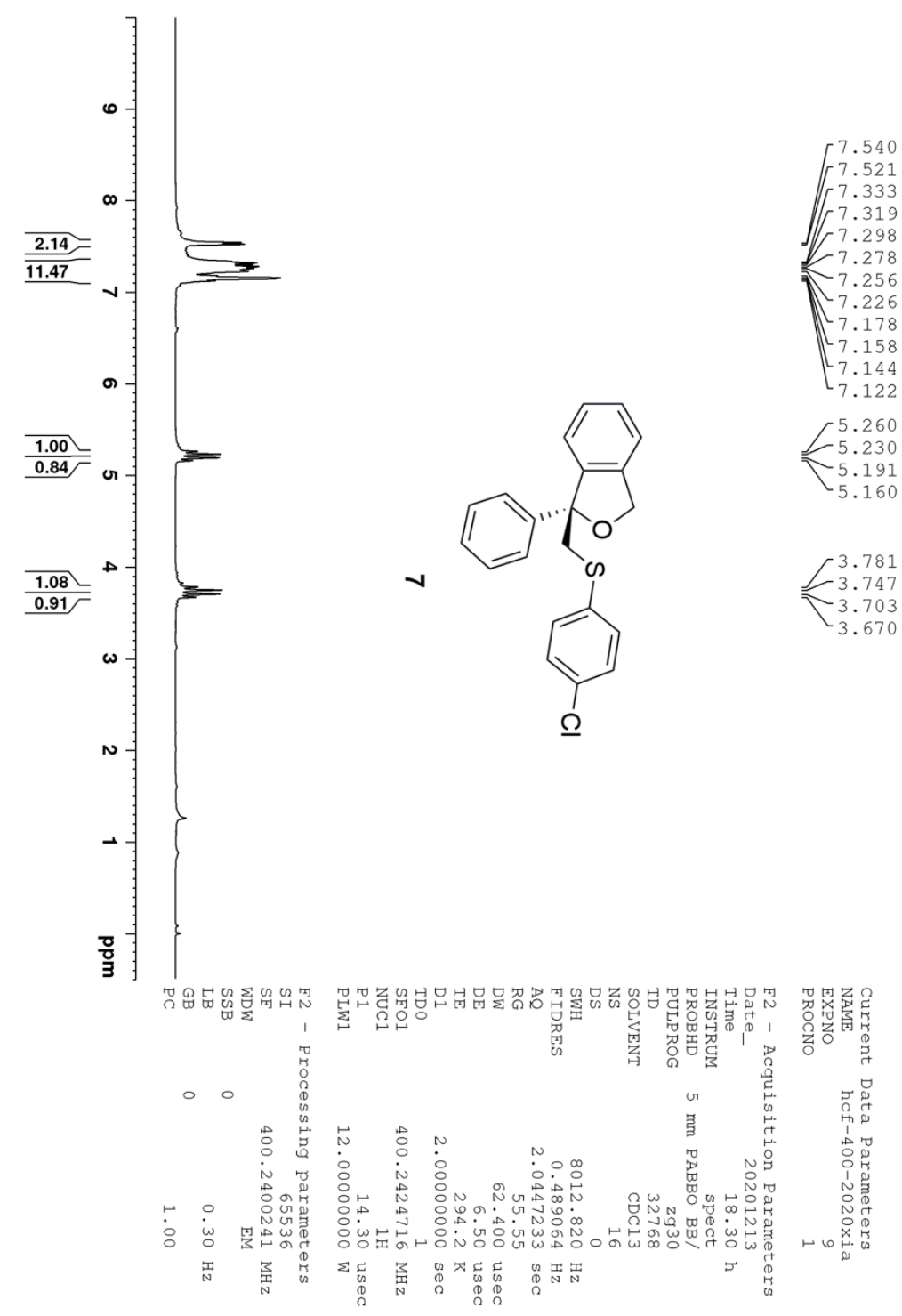




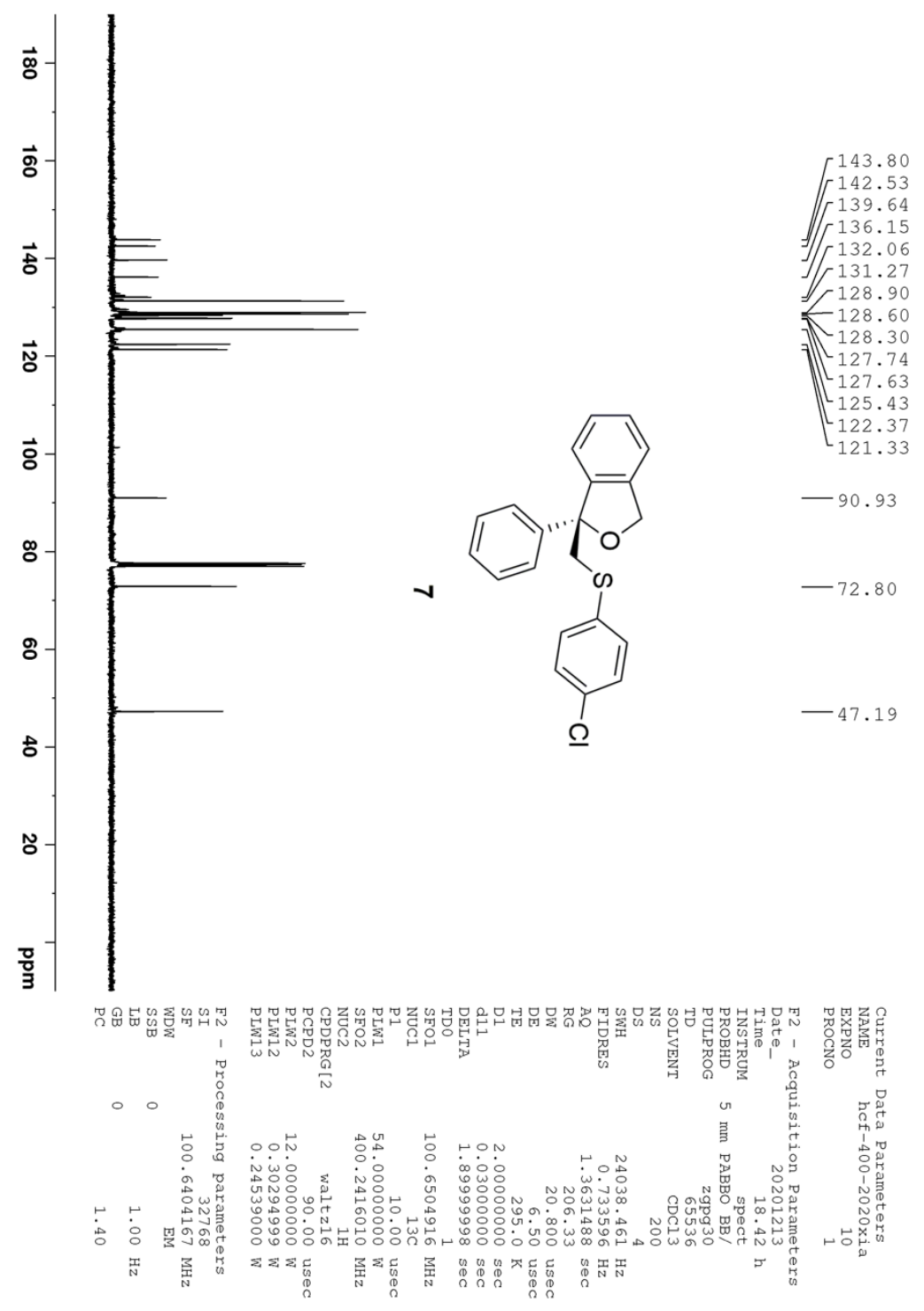




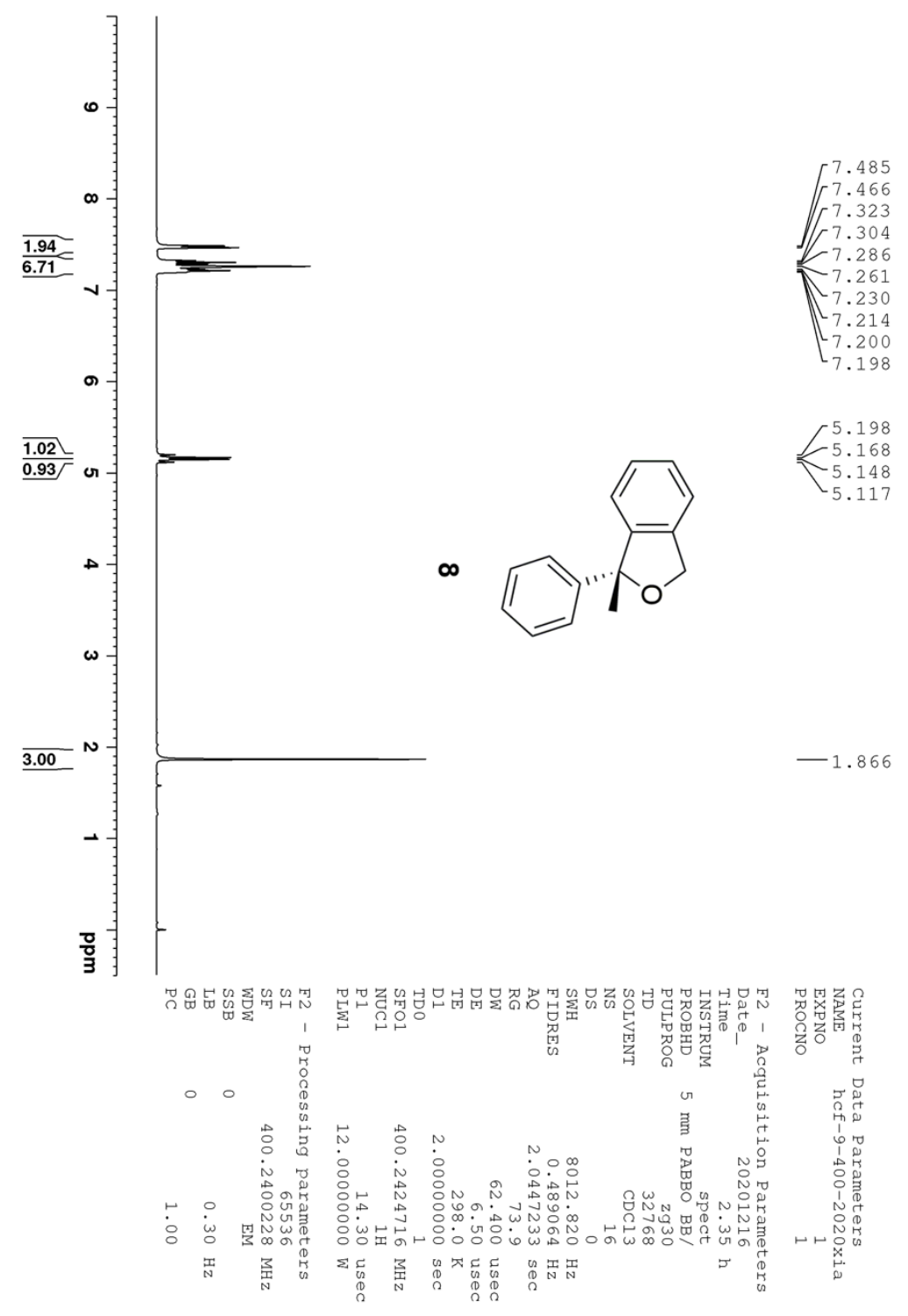




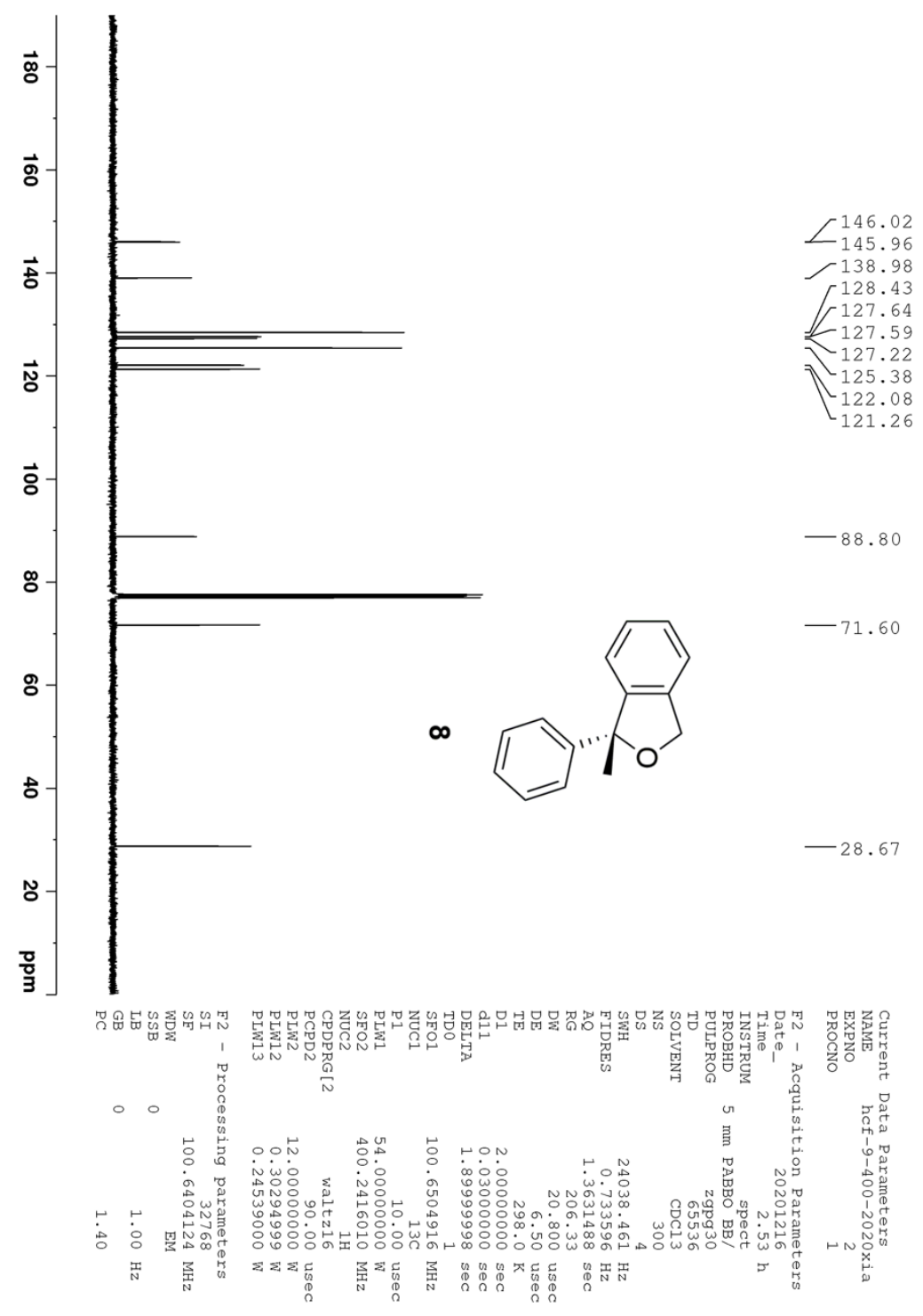




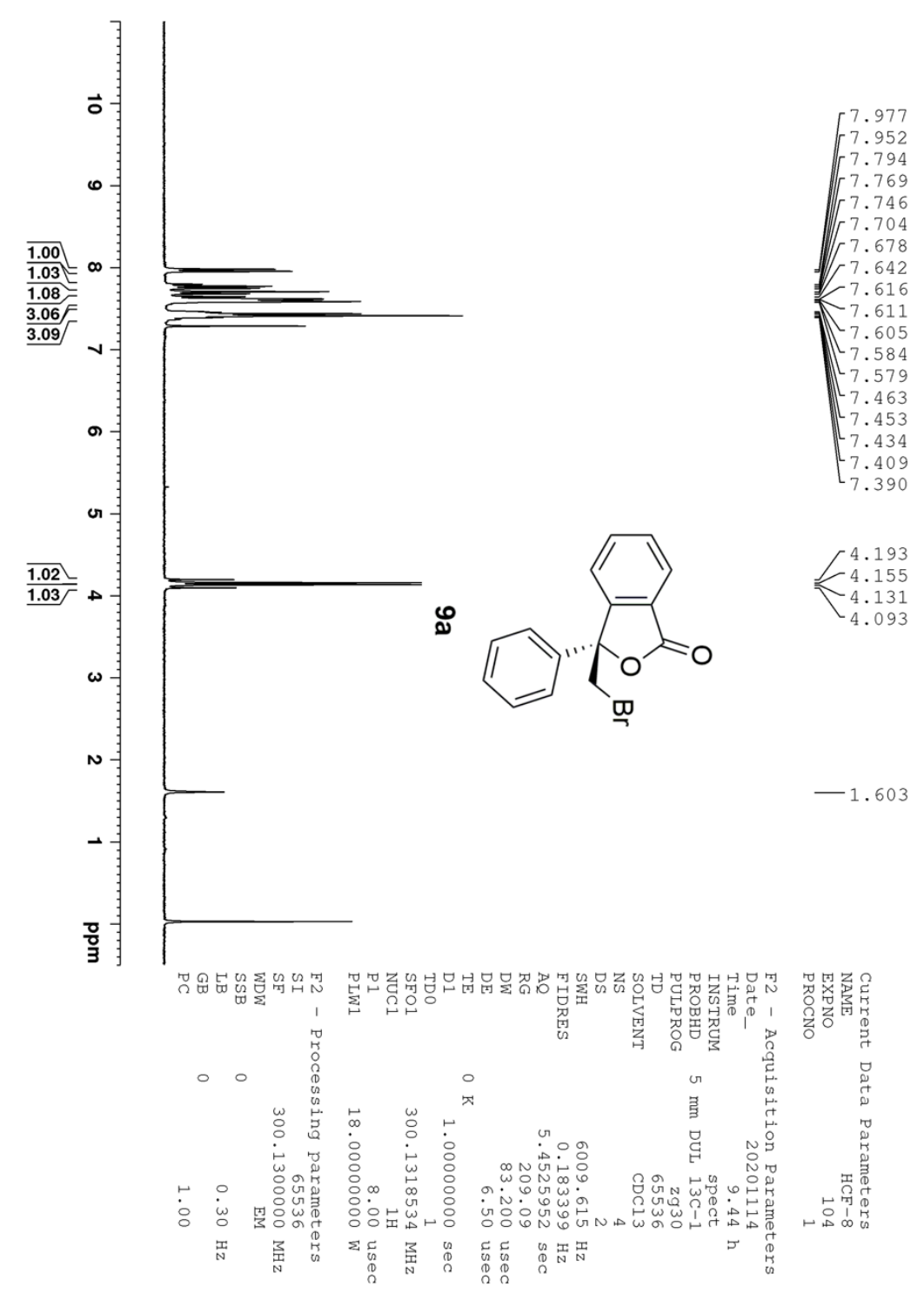




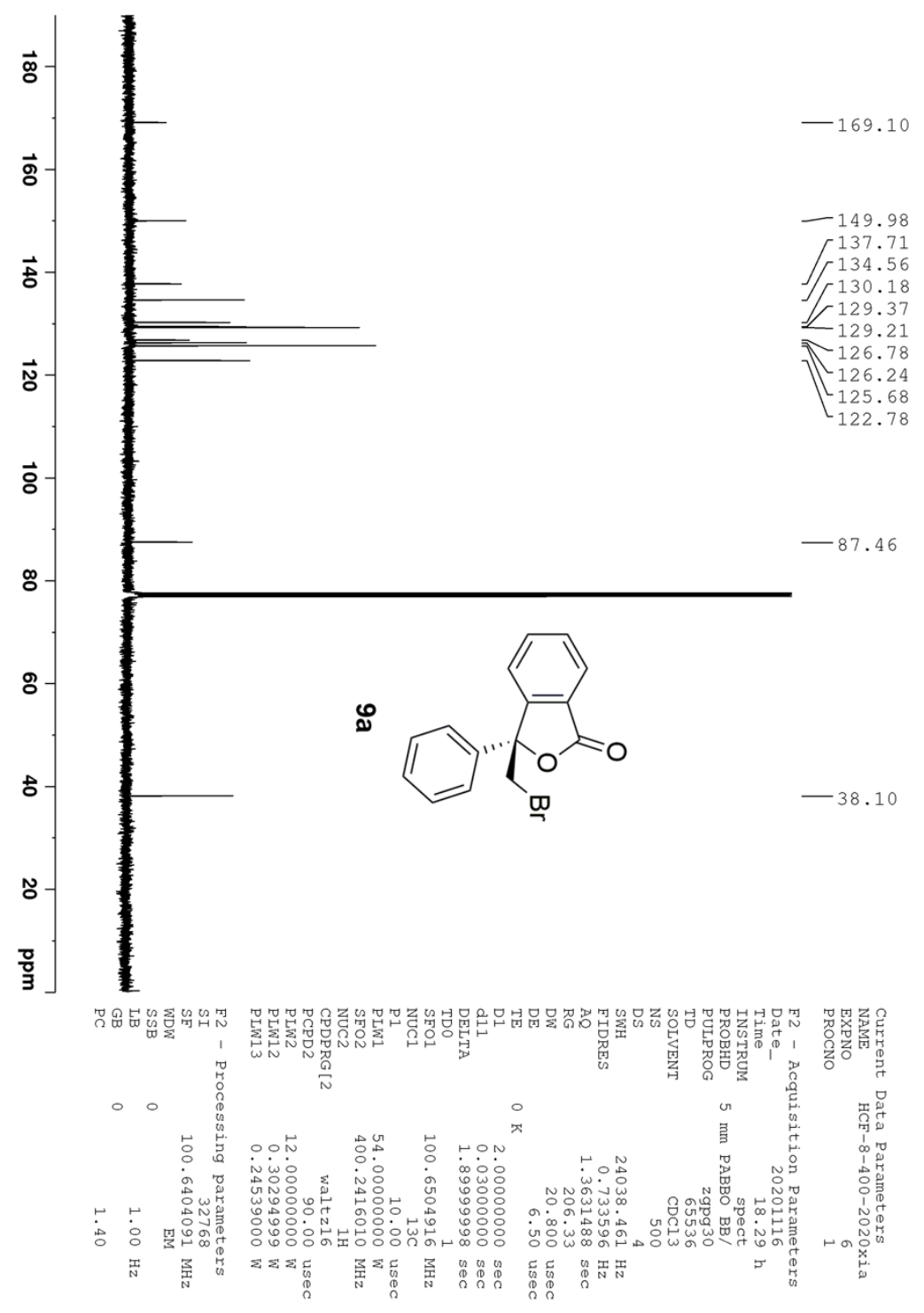




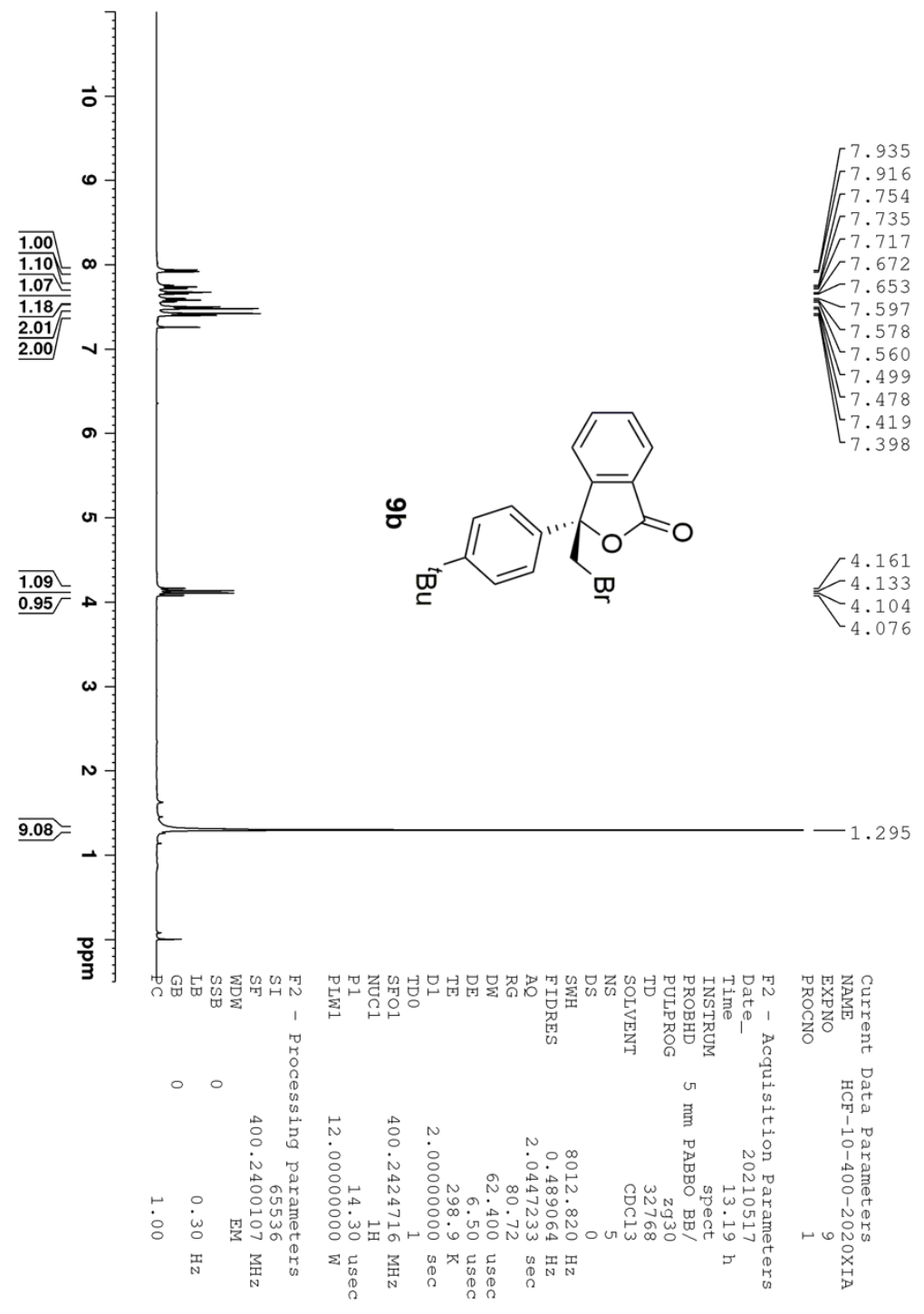




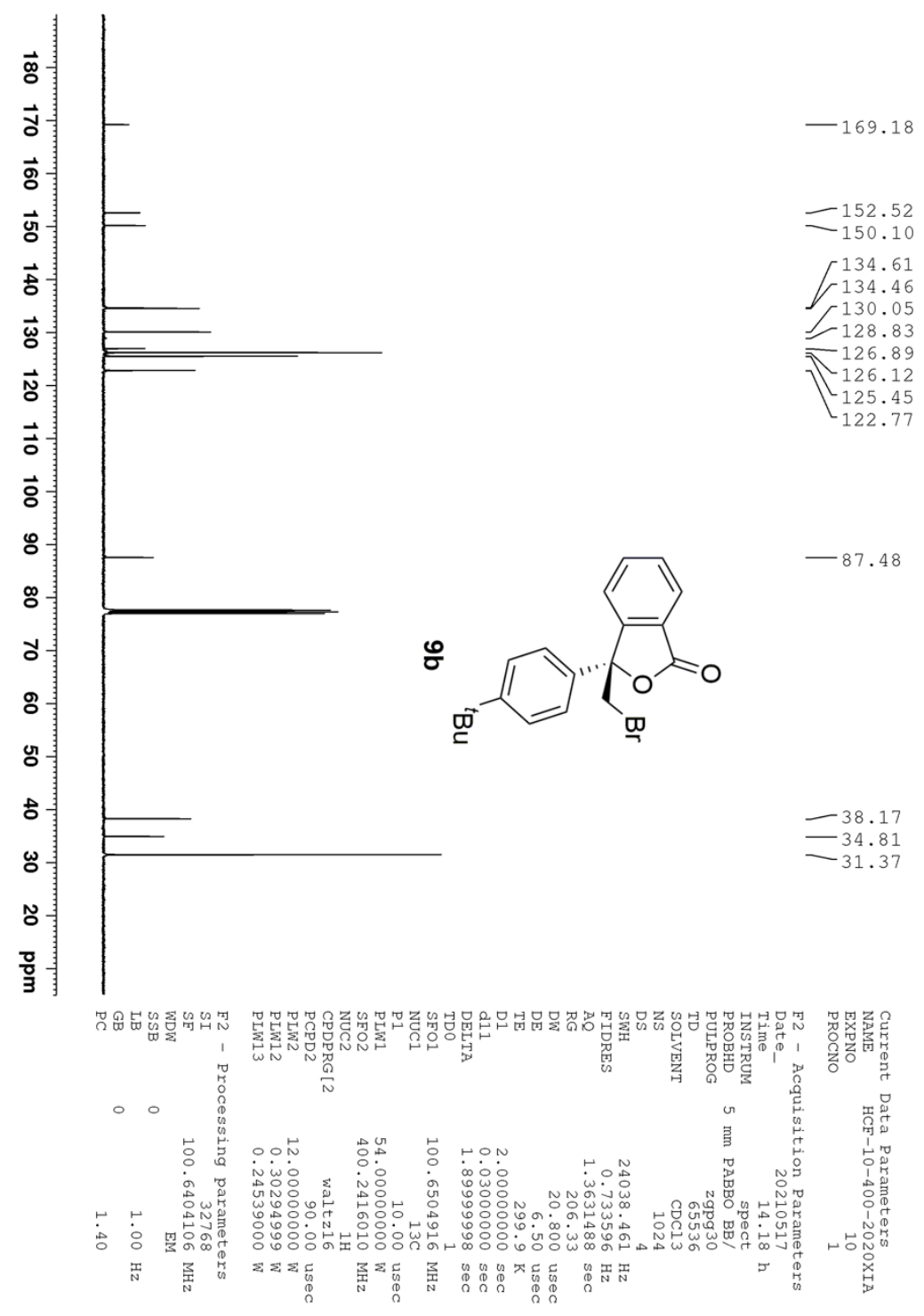




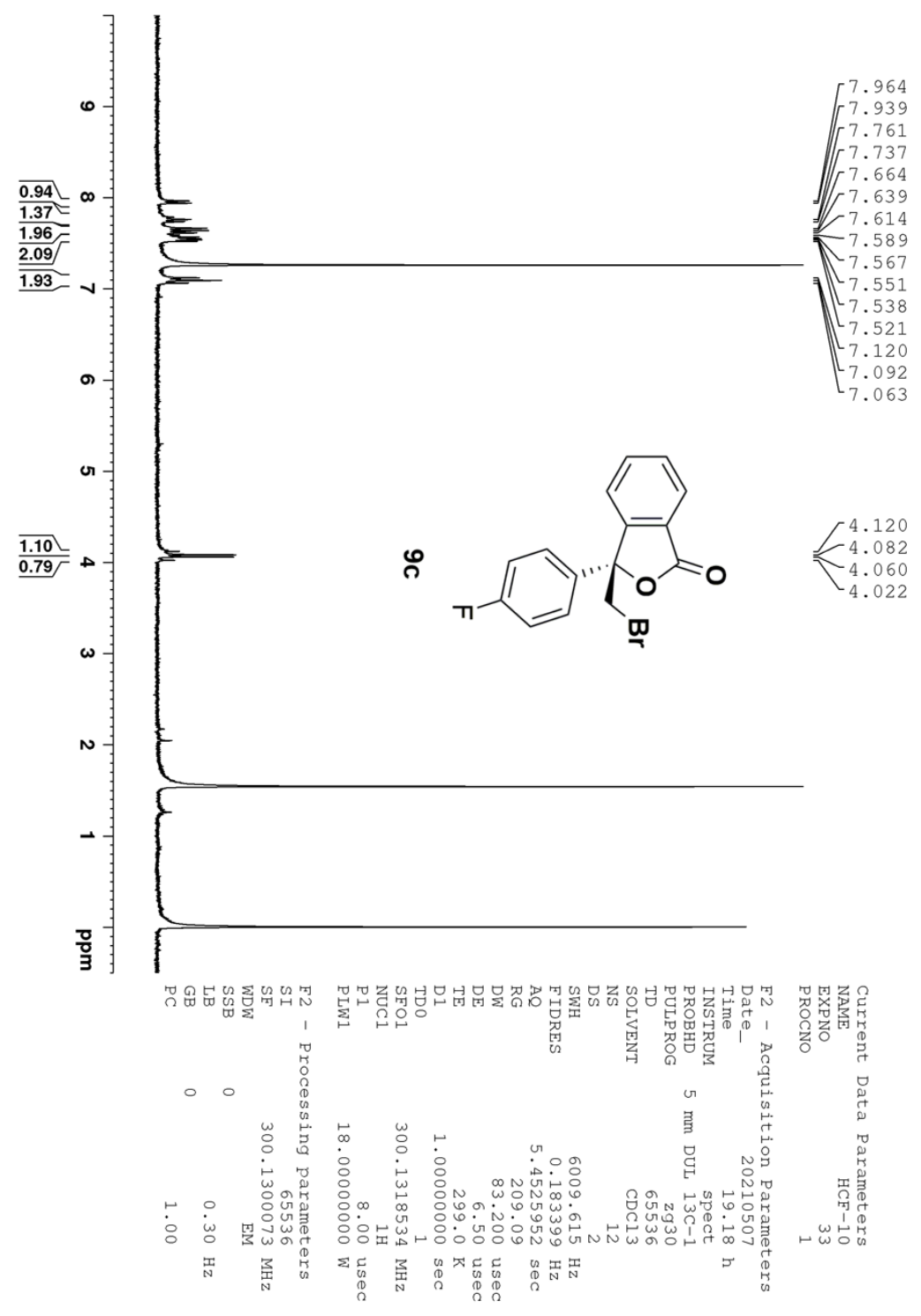




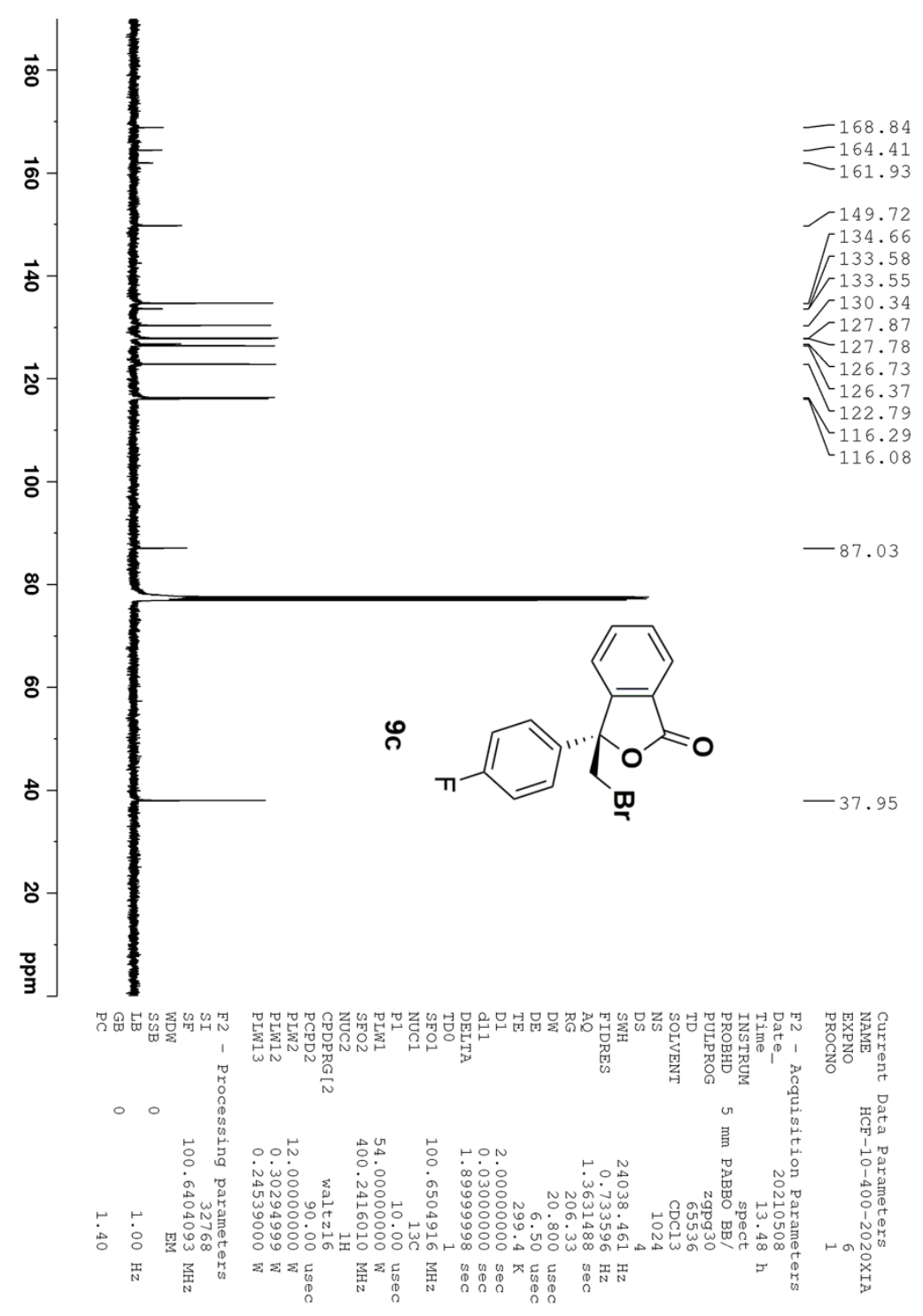




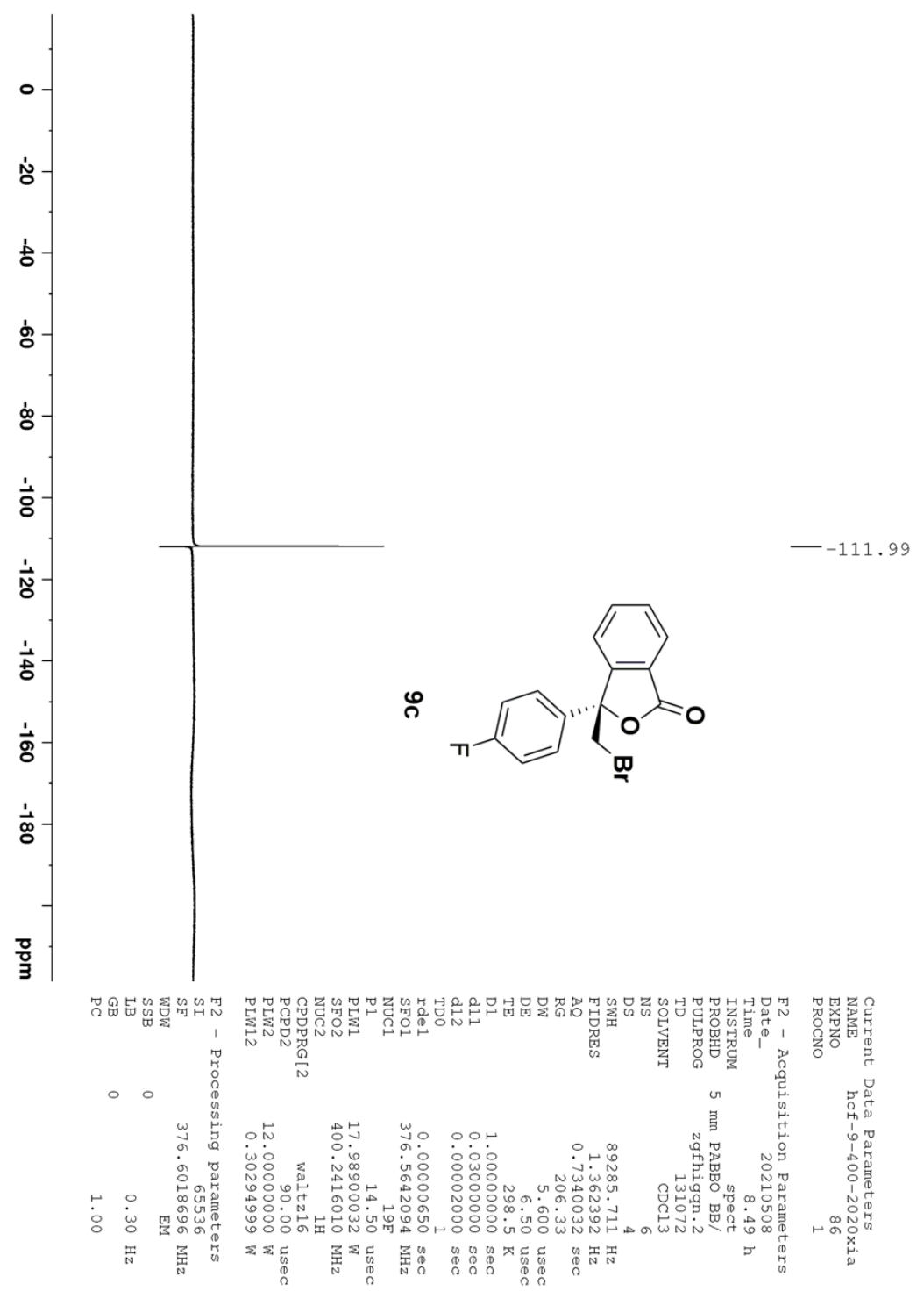




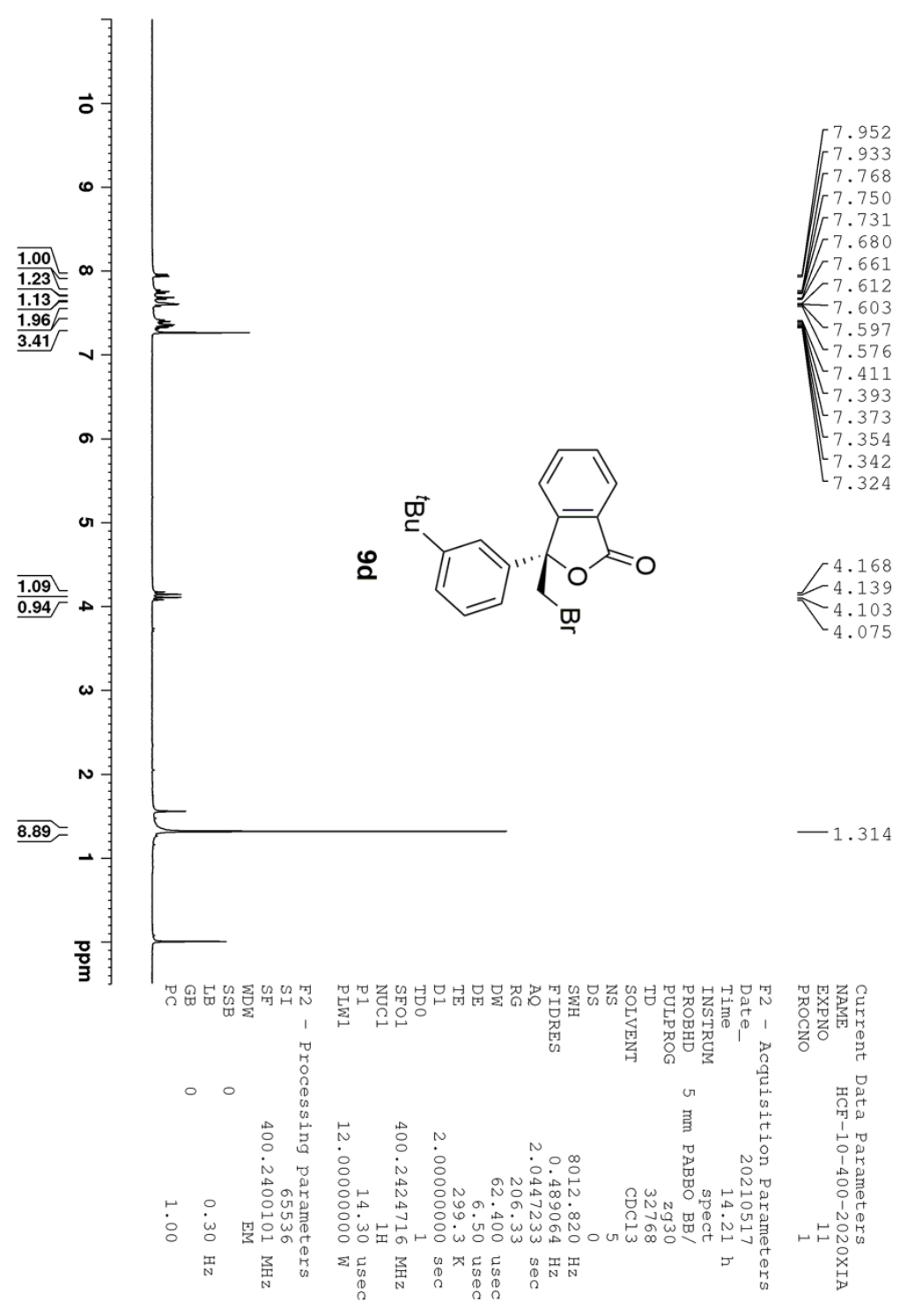




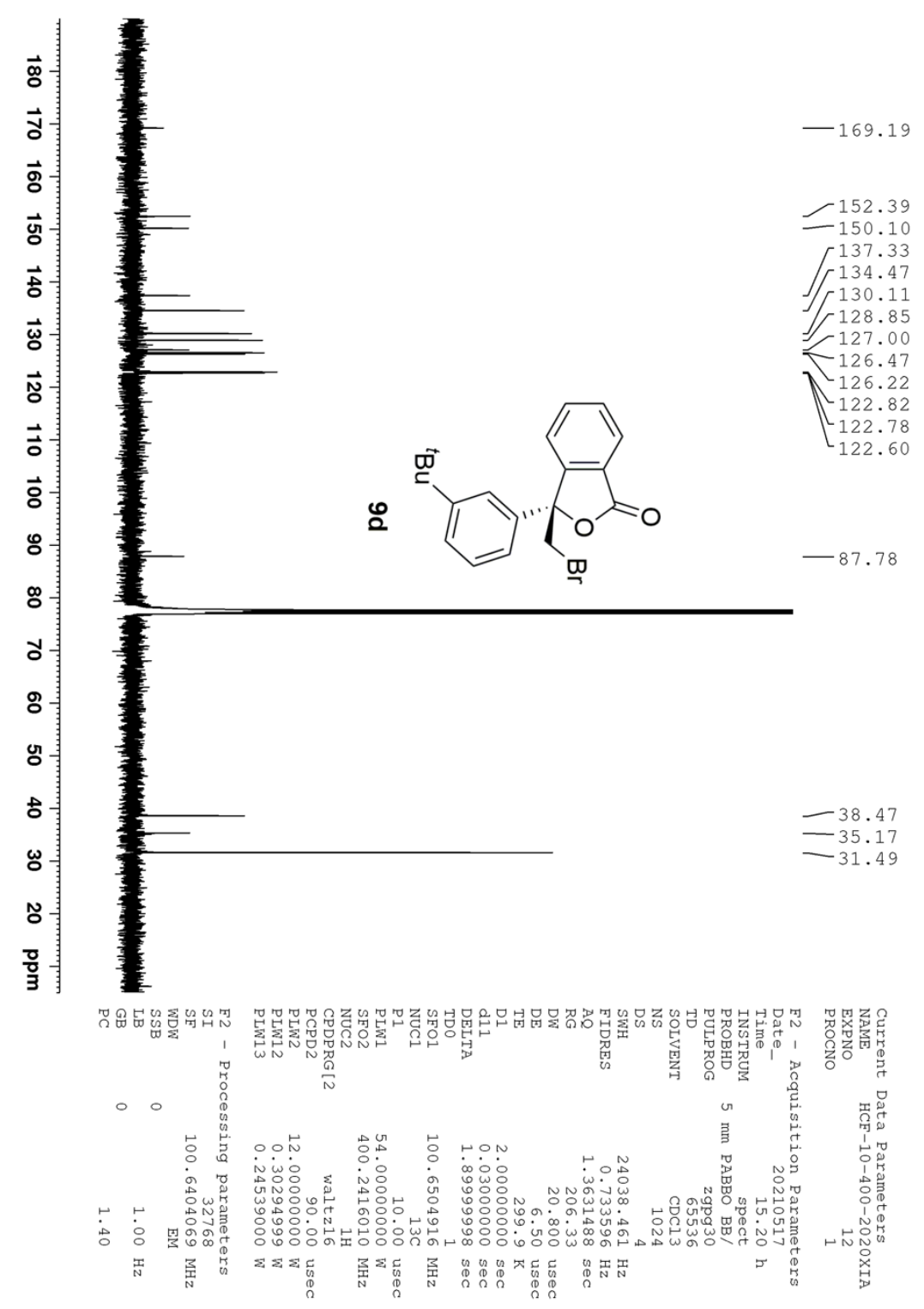




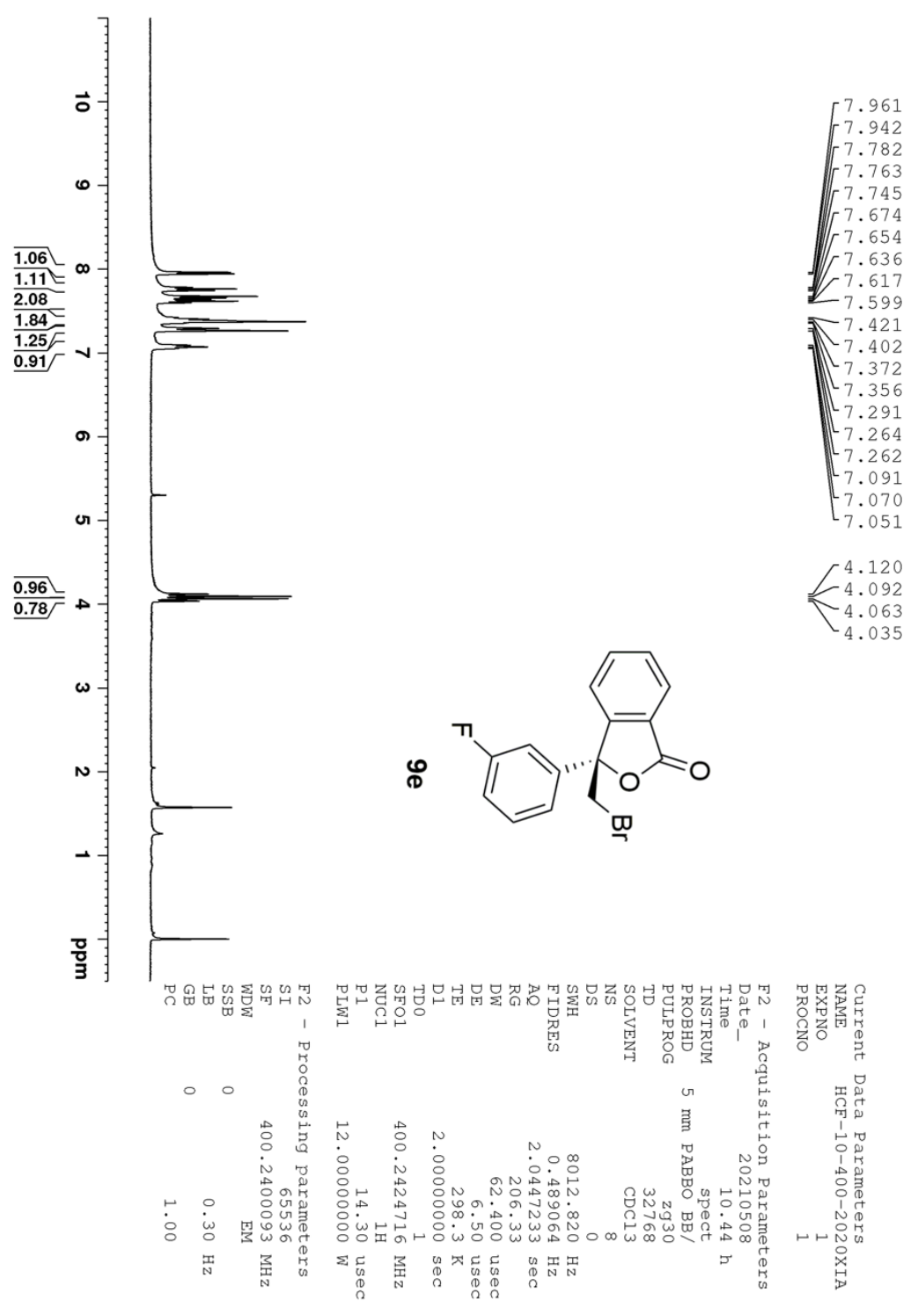




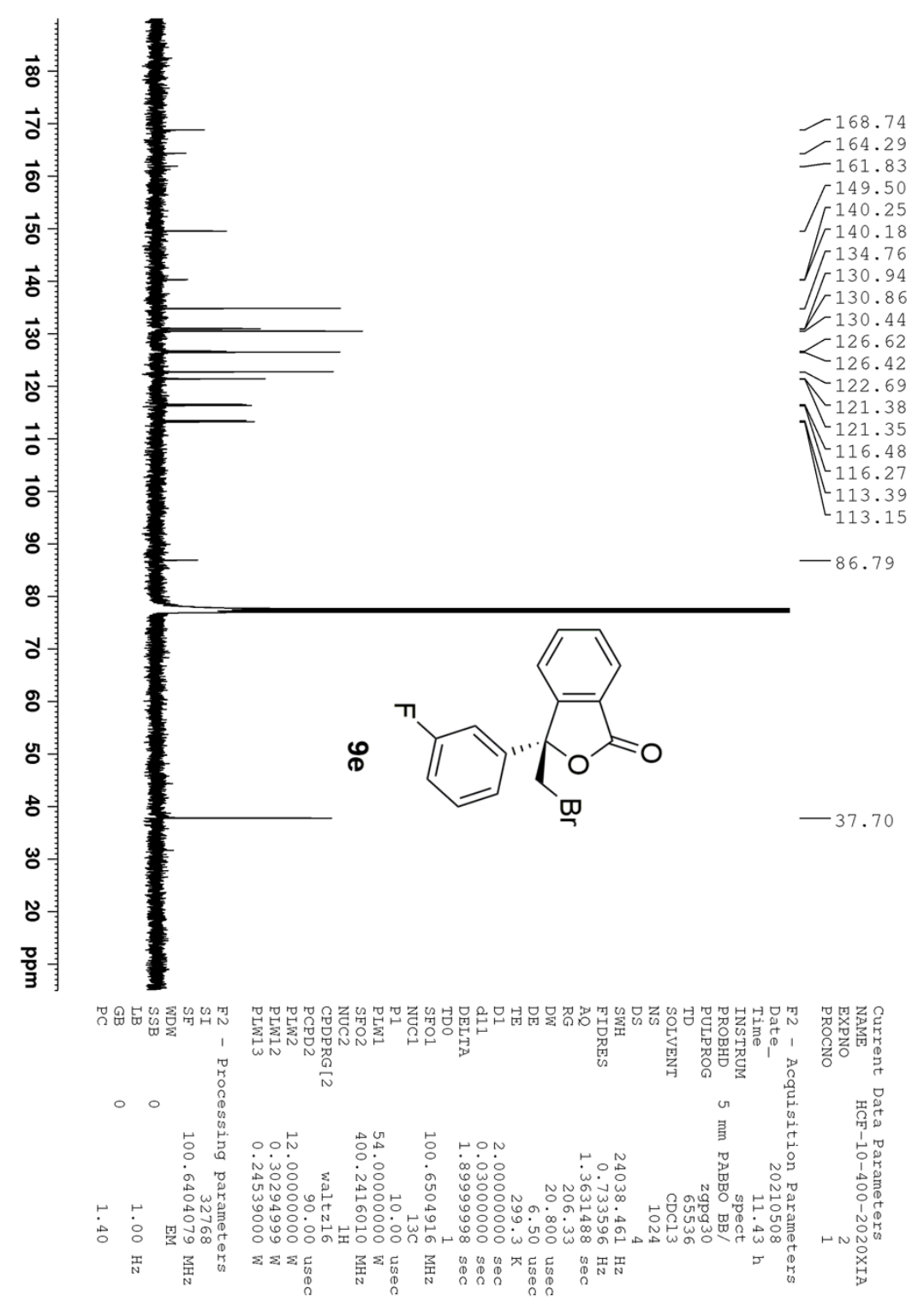




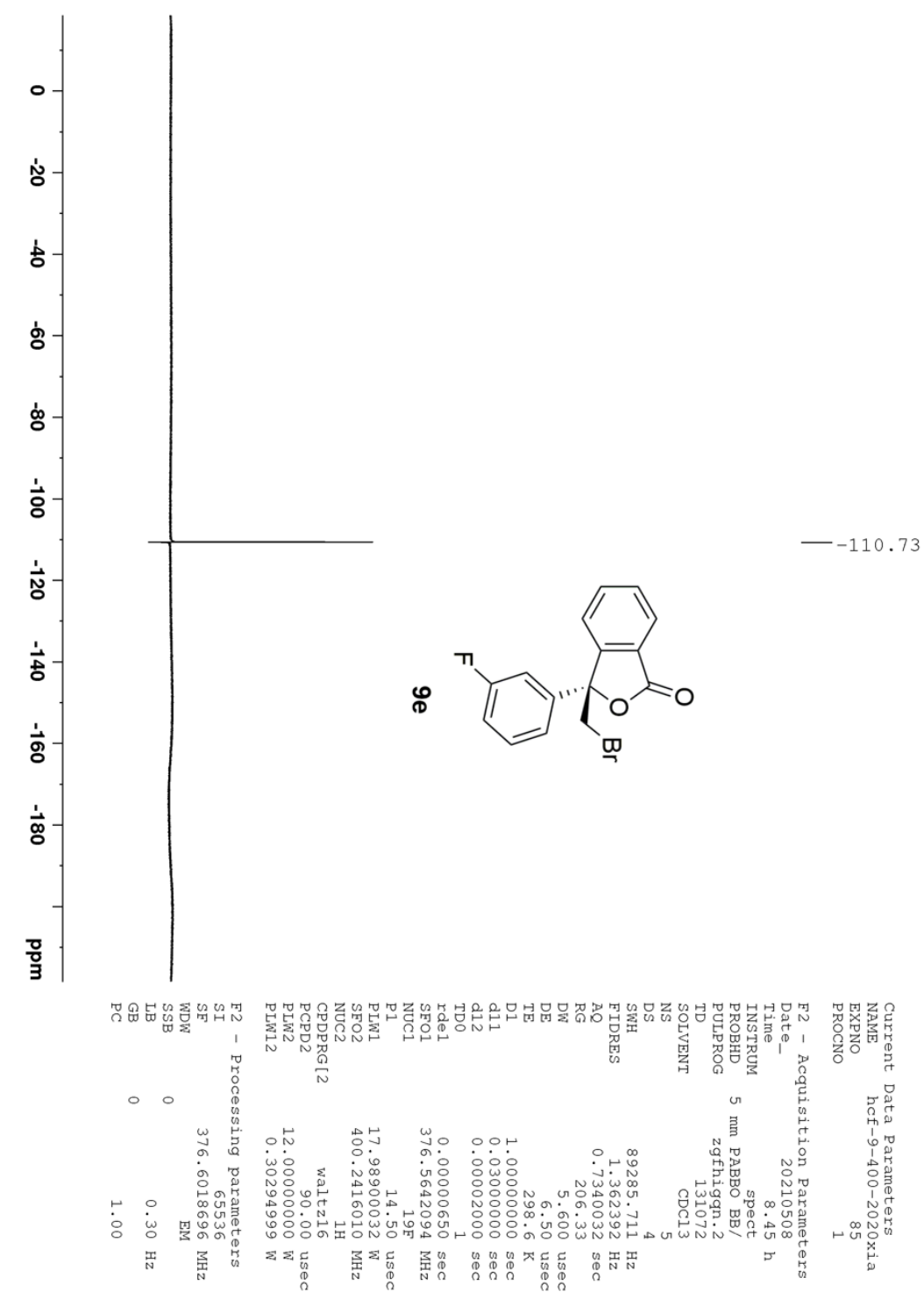




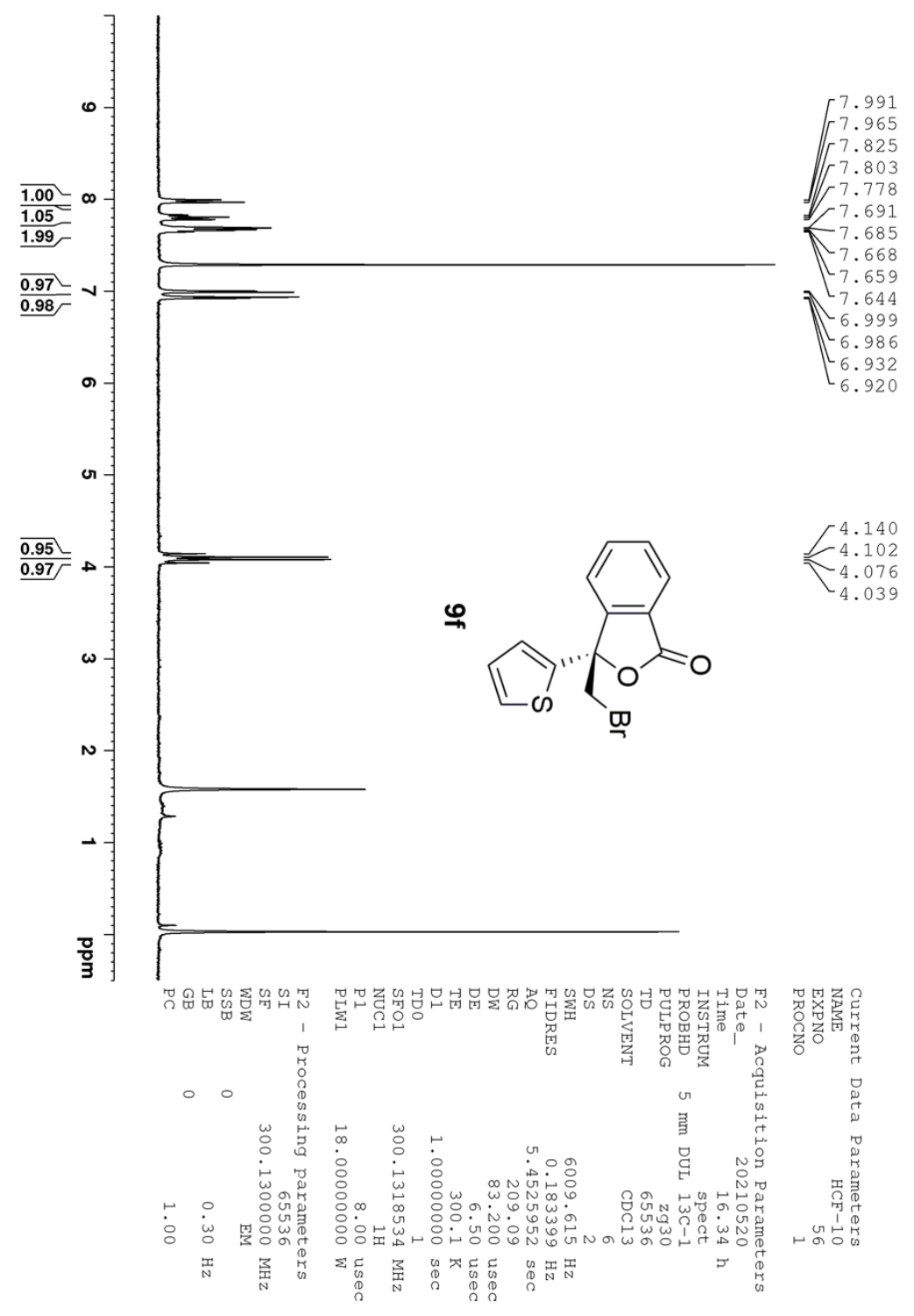




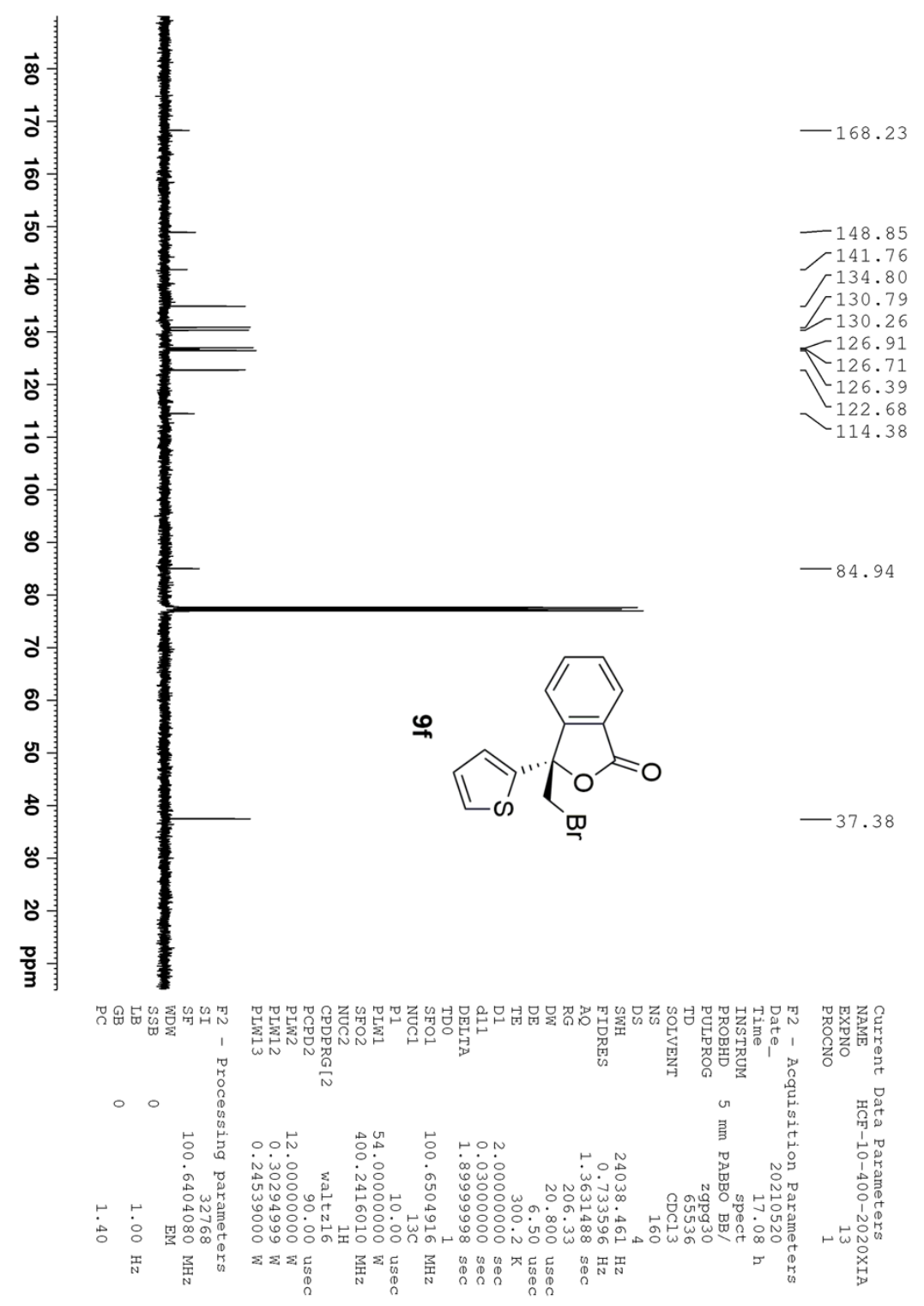

